\title{
Estimation of Nonlinear Greybox Models for Marine Applications
}

Fredrik Ljungberg

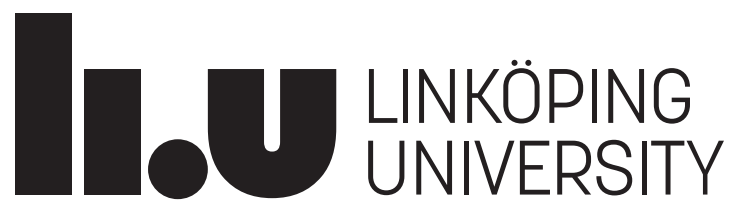




\section{Estimation of Nonlinear Greybox Models for Marine Applications}

Fredrik Ljungberg 
This is a Swedish Licentiate's Thesis.

Swedish postgraduate education leads to a Doctor's degree and/or a Licentiate's degree.

A Doctor's Degree comprises 240 ECTS credits (4 years of full-time studies).

A Licentiate's degree comprises 120 ECTS credits,

of which at least 60 ECTS credits constitute a Licentiate's thesis.

Linköping studies in science and technology. Licentiate Thesis

No. 1880

\section{Estimation of Nonlinear Greybox Models for Marine Applications}

Fredrik Ljungberg

fredrik. Ijungbergeliu.se

www. control.isy.liu.se

Department of Electrical Engineering

Linköping University

SE-581 83 Linköping

Sweden

ISBN 978-91-7929-840-1ＩSSN 0280-7971

Copyright (C) 2020 Fredrik Ljungberg

Printed by LiU-Tryck, Linköping, Sweden 2020 
To my family and friends! 



\section{Abstract}

As marine vessels are becoming increasingly autonomous, having accurate simulation models available is turning into an absolute necessity. This holds both for facilitation of development and for achieving satisfactory model-based control. When accurate ship models are sought, it is necessary to account for nonlinear hydrodynamic effects and to deal with environmental disturbances in a correct way. In this thesis, parameter estimators for nonlinear regression models where the regressors are second-order modulus functions are analyzed. This model class is referred to as second-order modulus models and is often used for greybox identification of marine vessels. The primary focus in the thesis is to find consistent estimators and for this an instrumental variable (IV) method is used.

First, it is demonstrated that the accuracy of an IV estimator can be improved by conducting experiments where the input signal has a static offset of sufficient amplitude and the instruments are forced to have zero mean. This two-step procedure is shown to give consistent estimators for second-order modulus models in cases where an off-the-shelf applied IV method does not, in particular when measurement uncertainty is taken into account.

Moreover, it is shown that the possibility of obtaining consistent parameter estimators for models of this type depends on how process disturbances enter the system and on the amount of prior knowledge about the disturbances' probability distributions that is available. In cases where the first-order moments are known, the aforementioned approach gives consistent estimators even when disturbances enter the system before the nonlinearity. In order to obtain consistent estimators in cases where the first-order moments are unknown, a framework for estimating the first and second-order moments alongside the model parameters is suggested. The idea is to describe the environmental disturbances as stationary stochastic processes in an inertial frame and to utilize the fact that their effect on a vessel depends on the vessel's attitude. It is consequently possible to infer information about the environmental disturbances by over time measuring the orientation of a vessel they are affecting. Furthermore, in cases where the process disturbances are of more general character it is shown that supplementary disturbance measurements can be used for achieving consistency.

Different scenarios where consistency can be achieved for instrumental variable estimators of second-order modulus models are demonstrated, both in theory and by simulation examples. Finally, estimation results obtained using data from a full-scale marine vessel are presented. 



\section{Populärvetenskaplig sammanfattning}

I takt med att marina farkoster blir mer autonoma ökar behovet av noggranna matematiska farkostmodeller. Modellerna behövs både för att förenkla utvecklingen av nya farkoster och för att kunna styra farkosterna autonomt med önskad precision. För att erhålla allmängiltiga modeller behöver olinjära hydrodynamiska effekter samt systemstörningar, främst orsakade av vind- och vattenströmmar, tas i beaktning. I det här arbetet undersöks metoder för att skatta okända storheter i modeller för marina farkoster givet observerad data. Undersökningen gäller en speciell typ av olinjära modeller som ofta används för att beskriva marina farkoster. Huvudfokus i arbetet är att erhålla konsistens, vilket betyder att de skattade storheterna ska anta rätt värden när mängden observerad data ökar. För det används en redan etablerad statistisk metod som baseras på instrumentvariabler.

Det visas först att noggrannheten i modellskattningsmetoden kan förbättras om datainsamlingsexperimenten utförs på ett sätt så att farkosten har signifikant nollskild hastighet och instrumentvariablernas medelvärde dras bort. Den här tvåstegslösningen påvisas vara fördelaktig vid skattning av parametrar i den ovan nämnda modelltypen, framför allt då mätosäkerhet tas i beaktning.

Vidare så visas det att möjligheten att erhålla konsistenta skattningsmetoder beror på hur mycket kännedom om systemstörningarna som finns tillgänglig på förhand. I fallet då de huvudsakliga hastigheterna på vind- och vattenströmmar är kända, räcker den tidigare nämnda tvåstegsmetoden bra. För att även kunna hantera det mer generella fallet föreslås en metod för att skatta de huvudsakliga hastigheterna och de okända modellparametrarna parallellt. Denna idé baserar sig på att beskriva störningarna som stationära i ett globalt koordinatsystem och att anta att deras effekt på en farkost beror på hur farkosten är orienterad. Genom att över tid mäta och samla in data som beskriver en farkosts kurs, kan man således dra slutsatser om de störningar som farkosten påverkas av. Utöver detta visas det att utnyttjande av vindmätningar kan ge konsistens i fallet med störningar av mer generell karaktär.

Olika scenarion där konsistens kan uppnås visas både i teori och med simuleringsexempel. Slutligen visas också modellskattningsresultat som erhållits med data insamlad från ett fullskaligt fartyg. 



\section{Acknowledgments}

First and foremost, I would like to thank my supervisor Assoc. Prof. Martin Enqvist for your enthusiastic support and guidance. I am sincerely grateful that you always find time for discussions, your inspiring ideas and feedback are invaluable. I would also like to thank my co-supervisor Prof. Svante Gunnarsson.

Working at the Division of Automatic Control is a real pleasure thanks to the friendly and stimulating work environment. For this I would again like to thank Assoc. Prof. Martin Enqvist and Prof. Svante Gunnarsson, this time in their respective roles as present and former head of division. I would also like to express my gratitude to Ninna Stensgård, thanks a lot for all your help with administrative issues.

A good work environment is primarily established by the people in it. Therefore, I would also like to thank all my current and former co-workers at the division. You really make working there enjoyable. A special thank you to Magnus Malmström, Daniel Arnström and Alberto Zenere, whose feedback greatly improved the manuscript of this thesis.

This work was supported by the Vinnova Competence Center LINK-SIC, which is a collaboration between industry and academia that encompasses several Swedish system-building companies. I would like to thank all the partners of the center and in particular $\mathrm{ABB}$, with which I have a close research collaboration. A special thank you is directed to Dr. Jonas Linder for acting in the role as an industry supervisor. You manage to always find time for answering my questions despite the geographical distance. To have someone with your expertise that covers both system identification and marine modelling available for research discussions is of immense value.

Finally, I would like to thank my family for always being there for me. Evelina, I can not thank you enough for your patience and encouragement. I love you! 



\section{Contents}

Notation

xiii

1 Introduction 1

1.1 Research motivation . . . . . . . . . . . . . . . . 1

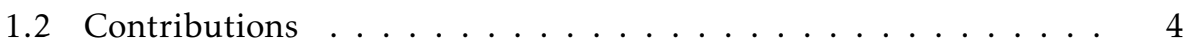

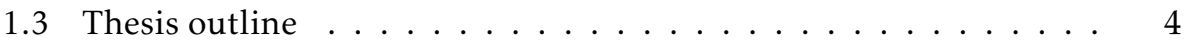

2 System Identification Preliminaries $\quad 7$

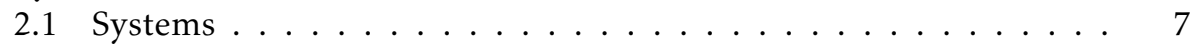

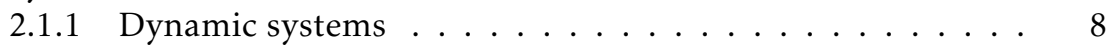

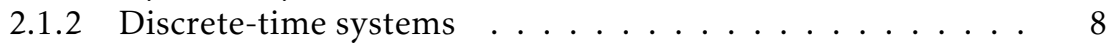

2.2 Models . . . . . . . . . . . . . . . . . . . 9

2.3 Parameter estimation . . . . . . . . . . . . . . . . 10

2.3.1 Prediction error methods . . . . . . . . . . . . . 11

2.3.2 Instrumental variable methods . . . . . . . . . . . 12

3 Marine Modelling 13

3.1 The undisturbed equations of motion . . . . . . . . . . 13

3.2 Environmental disturbances . . . . . . . . . . . . . . . . 15

3.3 Maneuvering models . . . . . . . . . . . . . . . . . 16

3.4 Nonlinear ship modelling . . . . . . . . . . . . . . . 17

3.4.1 Truncated Taylor expansion . . . . . . . . . . . 17

3.4.2 Second-order modulus models . . . . . . . . . . . 17

3.5 Deriving a model structure . . . . . . . . . . . . . . . . 18

3.5.1 Rigid-body kinetics . . . . . . . . . . . . . . . . . . 19

3.5 .2 Hydrodynamics . . . . . . . . . . . . . . . 20

3.5 .3 Aerodynamics . . . . . . . . . . . . . . . 21

3.5.4 Azimuth actuation . . . . . . . . . . . . . . 21

3.5 .5 State-space representation . . . . . . . . . . . . . 24

3.5 .6 Surge model . . . . . . . . . . . . . . . . 25

3.6 Estimating velocity states . . . . . . . . . . . . . . . . . 25

4 Eliminating Disturbances $\quad 31$ 


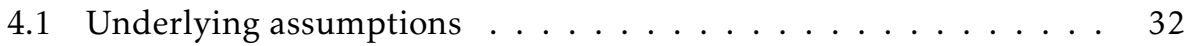

4.2 Zero-mean disturbance . . . . . . . . . . . . . . 34

4.3 General disturbance . . . . . . . . . . . . . . . . . . . . . 36

4.4 Motivating examples . . . . . . . . . . . . . . 39

4.5 Simulations ......................... 45

5 Estimating Disturbances $\quad 51$

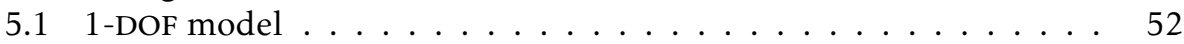

5.1 .1 Straight-line path motion . . . . . . . . . 53

5.1 .2 Augmenting the regression vector . . . . . . . . . . 55

5.1 .3 Violating the experiment condition .......... 58

5.1 .4 Including wind measurements . . . . . . . . . . . 60

5.2 Maneuvering model . . . . . . . . . . . . . . . 66

5.2 .1 The surge equation ............... 67

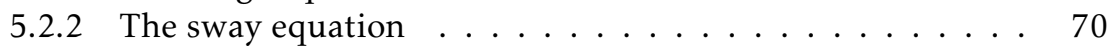

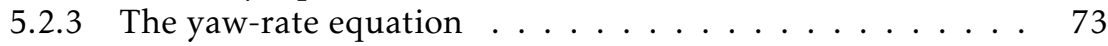

5.A Asymptotic model residuals . . . . . . . . . . . . . . 76

5.A.1 Surge equation - without wind measurements . . . . . . 76

5.A.2 Sway equation - without wind measurements . . . . . . 78

5.A.3 Sway equation - with wind measurements . . . . . . . 80

5.A.4 Yaw-rate equation - without wind measurements . . . . . 82

5.A.5 Yaw-rate equation - with wind measurements . . . . . 87

6 Simulation Study 91

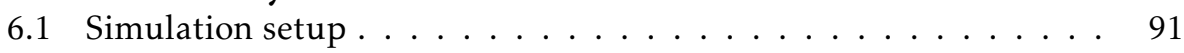

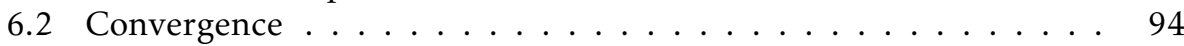

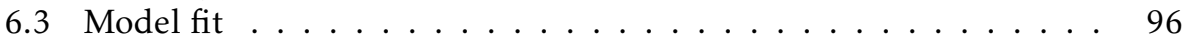

7 Experimental Study $\quad 107$

7.1 Experiment description . . . . . . . . . . . . . . . . 107

7.2 Parameter estimation ................... 108

8 Conclusions $\quad 119$

$\begin{array}{lr}\text { Bibliography } & 121\end{array}$ 


\section{Abbreviations}

\begin{tabular}{cl}
\hline Abbreviation & Meaning \\
\hline PEM & Prediction error method \\
LS & Least squares \\
IV & Instrumental variable \\
ML & Maximum likelihood \\
w.p. 1 & With probability 1 \\
SISO & Single-input single-output \\
DOF & Degrees of freedom \\
GNSS & Global navigation satellite system \\
\hline
\end{tabular}

\section{SigNALS AND SYSTEM IDENTIFICATION}

\begin{tabular}{cl}
\hline Variable & Description \\
\hline $\mathbf{u}(k)$ & Control signal \\
$\mathbf{y}(k)$ & Measured output \\
$\mathbf{e}(k)$ & Measurement noise \\
$\mathbf{w}(k)$ & Additive system disturbance \\
$\mathbf{v}(k)$ & Non-additive system disturbance \\
$\theta$ & Parameters in estimation problems \\
$\theta_{0}$ & True system parameters \\
$\hat{\mathbf{y}}(k \mid \theta)$ & The one-step-ahead predictor of $\mathbf{y}(k)$ \\
$\Phi(k)$ & The regression matrix \\
$\varphi(k)$ & Column of the regression matrix \\
$\mathbf{Z}(k)$ & The instrument matrix \\
$\zeta(k)$ & Column of the instrument matrix \\
\hline &
\end{tabular}




\section{REFERENCE FRAMES}

\begin{tabular}{cl}
\hline Frame & Description \\
\hline$b$-frame & Body-fixed reference frame, see Definition 3.1 \\
$n$-frame & World-fixed reference frame, see Definition 3.2 \\
\hline
\end{tabular}

Position AND ATtitude

\begin{tabular}{cl}
\hline Variable & Description \\
\hline$x_{n}(k)$ & Position relative the $n$-frame \\
$y_{n}(k)$ & Position relative the $n$-frame \\
$z_{n}(k)$ & Position relative the $n$-frame \\
$\phi(k)$ & Roll angle relative the $n$-frame \\
$\theta(k)$ & Pitch angle relative the $n$-frame \\
$\psi(k)$ & Yaw angle relative the $n$-frame \\
$\eta(k)$ & Vector with position and attitude states \\
\hline
\end{tabular}

\section{Generalized velocities}

\begin{tabular}{cl}
\hline Variable & Description \\
\hline$u(k)$ & Surge speed, velocity along the $x_{b}$-axis of the $b$-frame \\
$v(k)$ & Sway speed, velocity along the $y_{b}$-axis of the $b$-frame \\
$w(k)$ & Heave speed, velocity along the $z_{b}$-axis of the $b$-frame \\
$p(k)$ & Roll rate, velocity about the $x_{b}$-axis of the $b$-frame \\
$q(k)$ & Pitch rate, velocity about the $y_{b}$-axis of the $b$-frame \\
$r(k)$ & Yaw rate, velocity about the $z_{b}$-axis of the $b$-frame \\
$v(k)$ & Vector with generalized velocity states \\
\hline
\end{tabular}

\section{ENVIRONMENTAL DISTURBANCES}

\begin{tabular}{cl}
\hline Variable & Description \\
\hline$v_{c}(k)$ & Velocity of an ocean current \\
$v_{w}(k)$ & Velocity of the wind \\
$v_{r}(k)$ & Relative velocity with respect to an ocean current \\
$v_{q}(k)$ & Relative velocity with respect to the wind \\
\hline
\end{tabular}




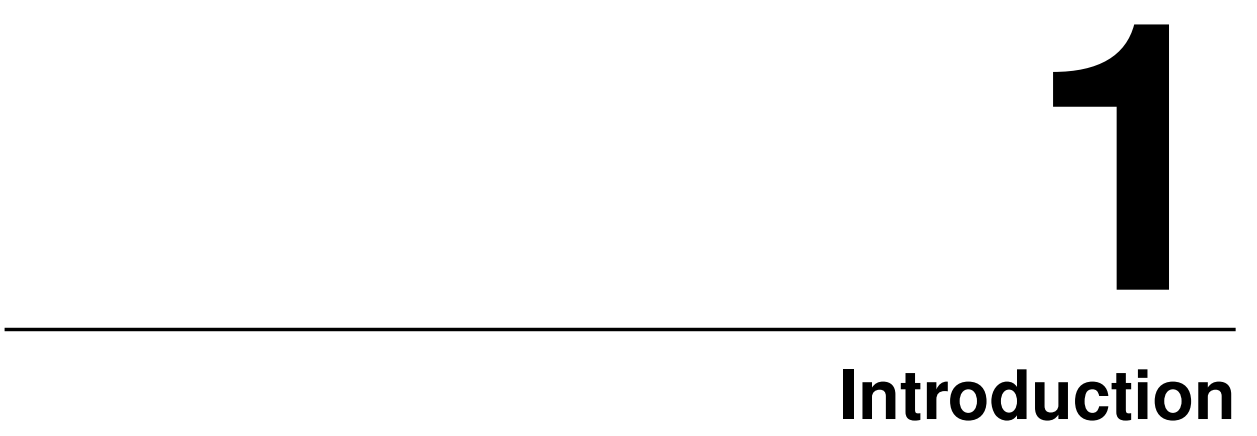

In this work, ways of finding mathematical models for marine vessels are explored. The modelling is done using measurements from onboard sensors and is a rather involved task. This is primarily due to the nonlinear dynamic forces and moments affecting the vessel. The forces and moments are primarily caused by interaction with the surrounding water but in the case of surface vessels, also by interaction with the surrounding air. This is especially true for vessels that expose a large side area to the wind, like container ships and cruise ferries. Generally, the two surrounding media will move with respect to each other which complicates things even further.

This introductory chapter contains background to the carried out work followed by descriptions of the scientific contributions of this thesis. Beyond this, the structure of the thesis is outlined.

\subsection{Research motivation}

Ship motion and control have engaged researchers for at least a century, see for example Minorsky [1922] for an early reference. Even if the controller concept has hardly changed since then, there have been great advances made. One major difference in modern-time automatic steering of ships, is that many control methods are model based. Therefore, as marine vessels are becoming increasingly autonomous having accurate simulation models available is turning into an absolute necessity. This holds both for facilitation of development and for achieving satisfactory control. In present time, it is also desired to automate more advanced maneuvers. Linear theory is useful for analyzing ship motion performed within close proximity to an equilibrium point, but it is not useful for accurately pre- 
dicting the characteristics of tight maneuvers, that are for example used during docking at ports. When general control solutions are sought, it is therefore necessary to account for nonlinear effects during modelling.

Ship dynamics depend on the forces and moments acting on the ship according to Newton's laws of motion. In addition to actuators, like thrusters and rudders, also environmental forces affect the steering dynamics in this way. Dealing with these, typically quite impactful process disturbances, in a correct way during model estimation is quite challenging already in the linear case and becomes even more difficult when models are nonlinear. In this work, tools from the research field of system identification are adopted for finding models. System identification is the study of data-based modelling of dynamical systems based on measurements of their input and output signals. If the measurement data is collected under presence of environmental disturbances and these disturbances are not accounted for during the model estimation, the resulting model might be biased. In practical terms, this means that instead of just describing the sought characteristics of the vehicle in question, the model can adapt to the weather conditions prevailing under the data acquisition. Moreover, there is always an uncertainty associated with measuring something. Dealing with this inherent uncertainty is also of importance in order to obtain accurate models.

Within the field of system identification, the challenges of parameter estimation for nonlinear model classes are widely known, see for example Ljung [2010]. As a consequence there is a substantial research effort focused on the problem. One possible way of approaching it is to consider cases where the Maximum Likelihood problem can be formulated and solved. In Schön et al. [2011], this was done using the Expectation Maximization algorithm and particle smoothing. In Abdalmoaty [2019], a prediction-error perspective with suboptimal predictors was explored. The results showed that linear predictors can give consistent estimators in a prediction-error framework, for a quite large class of nonlinear models. Larsson [2019] investigated the possibility of having a parameterized linear observer capturing unmodelled disturbance characteristics. This linear observer was an easily accessible way of compensating for miss-specified predictors. All these works deal with quite general model classes, whereas this work deals with a special type of nonlinear regression models. However, the ambition is that some insights gained in this work should assist in a better understanding of more general nonlinear system identification as well.

This work is not the first at applying system identification to marine applications. Classical techniques for system identification applied to ship maneuvering include the prediction error method [Zhou and Blanke, 1989], the extended Kalman filter [Fossen et al., 1996, Yoon and Rhee, 2003], and model reference adaption [Van Amerongen, 1984]. During the past decades, these techniques have been refined in several ways and more recently in Herrero and Gonzalez [2012], identification of a high-speed trimaran ferry was done using a nonlinear prediction-error method with the unscented Kalman filter. Moreover, in Perez et al. [2007] and Sutulo and Soares [2014], genetic algorithms were used to minimize measures of the difference between the reference response and the response 
obtained with the identified parameters. Another recently suggested technique for parameter estimation is support vector machine regression. This was applied to ships in Luo et al. [2016]. Special maneuvers based on steady-state relationships, known as circle tests, can also be employed. One work where a maneuver like this was used is Casado et al. [2007]. See Fossen [2011] for more examples regarding this kind of experiments.

Sometimes the unknown model parameters are obtained in non-data-driven ways. In Skjetne et al. [2004], a nonlinear model of a scale ship was obtained by first having some of the parameters being measured directly, during what is called towing tests. Very accurate parameter estimates can be obtained in this way but the experiments are often expensive and time consuming, especially when carried out in full scale. In Kopman et al. [2015], a nonlinear model of an underwater vehicle in the shape of a fish was developed. In that work, a nonlinear prediction-error method was used to find values for the parameters connected to the frontal part of the robotic fish whereas the tail was modelled using beam theory. Recently there have also been advances in development of methods using computational fluid dynamics for ship hydrodynamics. In Carrica et al. [2013], such a method was used to model maneuvers of both a model ship and its full-scale equivalent.

Two main approaches for dealing with nonlinearities in ship models exist in the literature. The first is using a truncated odd Taylor series expansion which was proposed by Abkowitz [1964]. Only odd terms are considered because the model must behave in the same way for positive and negative relative velocities due to ship symmetry. The models usually include nonlinear terms of orders one and three.

The second alternative was first proposed by Fedyaevsky and Sobolev [1964] and later by Norrbin [1970] and provides another nonlinear representation called second-order modulus models. The second-order modulus models do, as the name suggests, include second-order terms. The constraint that the model must be based on an odd function is resolved by including absolute values. These models are not necessarily continuously differentiable, and strictly speaking they can therefore not represent the physical system. Experience has however shown that they can describe the water's damping effects quite accurately and they are therefore often used anyway, see for example Skjetne et al. [2004].

In this work, focus is on developing accurate parameter estimators for secondorder modulus models. The work serves as a continuation of Linder [2017], where the instrumental variable method was successfully applied for estimating parameters in linear ship models. The goal is to contribute to the research field of system identification regarding parameter estimation for nonlinear model classes while at the same time complementing earlier investigations of marine modelling, primarily by putting focus on having consistent estimators. 


\subsection{Contributions}

There are three main contributions in the thesis. The first contribution is the suggestion of an experiment design where the input signal has a static offset of sufficient amplitude and the instruments in an instrumental variable method are forced to have zero mean. This two-step procedure is analyzed in Chapter 4 and shown to give consistent parameter estimators for second-order modulus models in cases where an off-the-shelf applied instrumental variable method does not. There it is also shown that non-additive disturbances with unknown first-order moments make consistency hard to achieve, even when the above-mentioned procedure is followed. This leads on to the second contribution, which is a method to estimate the first-order moments of system disturbances alongside the parameters of a second-order modulus model, something that is further explained in Chapter 5. Using this approach, it is possible to obtain consistent parameter estimators despite data being collected under affect of non-additive disturbances. The third contribution is experimental work, both in simulation and using collected ship data. This work is presented in Chapters 6 and 7.

The results in Chapter 4 have also been published in

Fredrik Ljungberg and Martin Enqvist. Obtaining consistent parameter estimators for second-order modulus models. IEEE Control Systems Letters, 3(4):781-786, 102019.

Fredrik Ljungberg and Martin Enqvist. Consistent parameter estimators for second-order modulus systems with non-additive disturbances. In Proceedings of the 21st IFAC World Congress, Berlin, Germany, 2020 (to appear).

In the first paper, performing experiments with excitation offset in combination with zero-mean instruments is suggested. The second paper deals with nonadditive system disturbances.

\subsection{Thesis outline}

In the first part of the thesis, brief theoretical introductions to a selection of topics are given. This part contains no new results but is relevant for understanding the later parts. In Chapter 2, some theoretical preliminaries to system identification are given whereas Chapter 3 serves as an introduction to ship modelling.

In Chapter 4, it is shown that the accuracy of an instrumental variable estimator for second-order modulus models can be improved by conducting experiments where the input signal has a static offset of sufficient amplitude and the instruments are forced to have zero mean. It is also shown that the possibility of obtaining consistent parameter estimators for these models depends on how process disturbances enter the system. Two scenarios where consistency can be achieved 
for instrumental variable estimators despite non-additive system disturbances are demonstrated. This is first done in theory and then verified by small-scale simulation examples.

In Chapter 5, another method of obtaining consistent parameter estimators for second-order modulus models in the case of non-additive disturbances is explored. The main idea is to augment the regression vector with elements that capture the behavior of the noise distribution, i.e. to estimate the first and secondorder moments of the disturbances alongside the model parameters. This approach is shown to give consistency even when the disturbances have unknown first-order moments.

In order to illustrate the potential of the estimators derived throughout Chapters 4 and 5, simulations were performed. In Chapter 6, the results of these simulations are described.

In Chapter 7, estimation results obtained using data provided by ABB from a fullscale marine vessel are presented. First, the studied ship and the experiments are described briefly. Then, results from using the ship data for estimation of a maneuvering model derived in Chapter 3 are presented.

Finally, the thesis is concluded in Chapter 8. There, some ideas for future work are also listed. 



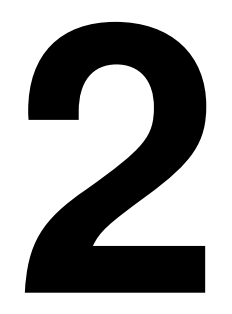

\section{System Identification Preliminaries}

System identification is the study of data-based modelling of dynamical systems based on measurements of their input and output signals. This is an important topic within the research field of automatic control, since many modern controldesign methods are model-based. System identification is a vast field of research and the aim of this chapter is not to provide the reader with a complete overview. Instead, focus is on a selection of topics that will be useful for understanding the remainder of the thesis.

\subsection{Systems}

The term system refers to an object within which several variables interact to produce observable effects. These effects are called output signals. The output signals, $\mathbf{y}$, are interesting because they are assumed to reflect the behavior of the system under the effect of external stimuli. The stimuli variables are called input signals and can be further divided into control signals, $\mathbf{u}$, which can be manipulated by a user of the system and disturbances, $\mathbf{w}$, which can not. Sometimes the disturbances can be measured, whereas other times they can only be observed through their influence on the output signals.

For discussions about modelling it is convenient to introduce the notion of a true system. The true system will be viewed as a mathematical mapping between the inputs and the outputs

$$
\mathbf{y}=f_{0}(\mathbf{u}, \mathbf{w})+\mathbf{e} .
$$

The additive variable, $\mathbf{e}$, represents the unavoidable uncertainty associated with measuring something. Whether nature is in reality explainable by a mathemat- 
ical function or not is a fundamental philosophical question which will not be explored in this thesis. The main use of the true system will be evaluation of modelling tools.

\subsubsection{Dynamic systems}

Generally, the output of a system does not only depend on the current value of the input but also on its historical values. Systems with this property are referred to as dynamic systems. The mapping from input to output is then given as a differential equation with respect to time. It will be assumed that all dynamic systems are causal, i.e. that the output does not depend on future values of the controlled input and disturbances. Then the output at a time instant $t$, does not depend on any signal at a time later than $t$.

A convenient way of expressing a causal dynamic system is the state-space representation

$$
\begin{aligned}
& \dot{\mathbf{x}}(t)=f\left(t, \mathbf{x}(t), \mathbf{u}(t), \mathbf{w}(t), \theta_{0}\right), \\
& \mathbf{y}(t)=h\left(t, \mathbf{x}(t), \mathbf{u}(t), \theta_{0}\right)+\mathbf{e}(t)
\end{aligned}
$$

Here $\mathbf{x}(t)$ is a latent variable which is referred to as the system state and $\theta_{0}$ is a vector of parameters that does not vary over time. The dot-notation indicates firstorder differentiation with respect to time. If there is no explicit time dependence in the functions $f$ and $h$, the system is said to be time invariant. Moreover, if $f$ and $h$ are linear functions in $\mathbf{x}(t), \mathbf{u}(t)$ and $\mathbf{w}(t)$, the system is said to be linear.

Linear and time-invariant dynamical systems constitute a well-studied special case for which (2.2) may be written in matrix form

$$
\begin{aligned}
& \dot{\mathbf{x}}(t)=A\left(\theta_{0}\right) \mathbf{x}(t)+B\left(\theta_{0}\right) \mathbf{u}(t)+N\left(\theta_{0}\right) \mathbf{w}(t), \\
& \mathbf{y}(t)=C\left(\theta_{0}\right) \mathbf{x}(t)+D\left(\theta_{0}\right) \mathbf{u}(t)+\mathbf{e}(t)
\end{aligned}
$$

A more general case is nonlinear and time-invariant systems

$$
\begin{aligned}
& \dot{\mathbf{x}}(t)=f\left(\mathbf{x}(t), \mathbf{u}(t), \mathbf{w}(t), \theta_{0}\right), \\
& \mathbf{y}(t)=h\left(\mathbf{x}(t), \mathbf{u}(t), \theta_{0}\right)+\mathbf{e}(t) .
\end{aligned}
$$

The systems studied in this work fall into this category.

\subsubsection{Discrete-time systems}

So far, the system representations have been given in continuous time. For many applications this is natural, because most known basic relationships are expressed in terms of differential equations. However, the only way to observe a system is by measurements and these are generally obtained as a finite collection of values, i.e. in discrete time. There are many ways to transform a continuous-time 
system representation like (2.4), to a discrete-time approximation. The simplest is perhaps Euler's explicit method. This method is based on a finite-difference approximation of the derivative

$$
\dot{\mathbf{x}}\left(k T_{s}\right) \approx \frac{1}{T_{S}}\left(\mathbf{x}\left(k T_{s}+T_{s}\right)-\mathbf{x}\left(k T_{s}\right)\right), k=1,2, \ldots
$$

Here $T_{S}$ is the time difference between two consecutive samples and $k T_{s}$ denotes the measurement sampling instants. Substituting the approximation into (2.4) gives

$$
\begin{aligned}
\mathbf{x}\left(k T_{s}+T_{s}\right) & \approx \mathbf{x}\left(k T_{s}\right)+T_{s} f\left(\mathbf{x}\left(k T_{s}\right), \mathbf{u}\left(k T_{s}\right), \mathbf{w}\left(k T_{s}\right), \theta_{0}\right) \\
& \triangleq f_{d}\left(\mathbf{x}\left(k T_{s}\right), \mathbf{u}\left(k T_{s}\right), \mathbf{w}\left(k T_{s}\right), \theta_{0}\right), \\
\mathbf{y}\left(k T_{s}\right) & =h\left(\mathbf{x}\left(k T_{s}\right), \mathbf{u}\left(k T_{s}\right), \theta_{0}\right)+\mathbf{e}\left(k T_{s}\right) .
\end{aligned}
$$

If $T_{S}$ is small with respect to the signal variations of $\mathbf{x}(t), \mathbf{u}(t)$ and $\mathbf{w}(t)$, the approximation will be accurate. Subsequently, the time indices $t$ and $k$ will be used to distinguish between continuous and discrete-time systems. Also, for simplified notation it will often be assumed that $T_{s}=1$.

\subsection{Models}

For successfully interacting with a system, it is necessary to make predictions about its behavior. This requires figuring out how the system variables relate to each other. Such an approximation of the true system will be referred to as a model. A model can sometimes be derived solely based on physical laws and prior knowledge about the system. This is called whitebox modelling. In other cases, a model can be based on collected measurement data. Let

$$
\mathcal{D}(N)=(\mathbf{y}(k), \mathbf{u}(k), \mathbf{o}(k))_{k=1}^{N},
$$

be a collection of $N$ data points. In addition to measurements of the output and control signals, the dataset, $\mathcal{D}(N)$, may include supplementary measured signals, $\mathbf{o}(k)$. This can for example be measurements of disturbances. There are many ways to characterize a mathematical model. In this work the one-step-ahead predictor

$$
\hat{\mathbf{y}}(k \mid \theta)=g(\mathcal{D}(k-1), \theta)
$$

will be used for this. The nonlinear filter $g($.$) takes as input previous data, \mathcal{D}(k-1)$, and the parameter vector $\theta$.

The identification problem is to find a predictor model which outputs are similar to those of the true system. Usually, this search is carried out over a set of candidate models. Except for the parameter vector, the predictor will depend 
on the underlying form of the filter $g($.$) . This underlying form will be referred$ to as the model structure. If no prior knowledge about a system is used when deciding upon a model structure, the identification procedure is called blackbox modelling. This is the straight opposite of whitebox modelling. Generally, any modelling in the area between both extremes is referred to as greybox modelling. There are many levels of greybox modelling and in Schoukens and Ljung [2019] a whole palette is defined. Common for all sorts of greybox modelling is that physical knowledge is first used to the extent possible. The remaining free elements are then adapted to a collected dataset. See for example Bohlin [2006] for a comprehensive treatment of greybox identification of industrial processes.

\subsection{Parameter estimation}

Once a model structure has been chosen, the parameters, $\theta$, can be determined by solving an optimization problem

$$
\hat{\theta}_{N}=\underset{\theta \in \vartheta}{\operatorname{argmin}} V_{N}(\mathcal{D}(N), \theta),
$$

where $V_{N}($.$) is a value function that depends on the data and on the chosen struc-$ ture. The search for an optimal $\theta$ is carried out over $\vartheta$, which is assumed to be an open subset of the real numbers. An optimization problem like (2.9) will be referred to as en estimator of $\theta$. Generally, each way of forming the value function, $V_{N}($.$) , will correspond to a unique estimator. In this work, different estimators$ will be compared.

A natural way of evaluating estimators is to compare their ability to converge to the values of the true system parameters $\theta_{0}$. However, given a model structure and a dataset it might be hard do find a reasonable estimator that actually do so. The issue depends both on the chosen model structure and whether the dataset is informative enough to distinguish between different models, as explained by Gevers et al. [2009]. If the data is sufficiently informative, the question remaining is whether the model structure is identifiable, i.e. if different $\theta$ can correspond to the same model.

Definition 2.1 (Global identifiability). A model structure $g($.$) is globally iden-$ tifiable if

$$
\left\{\begin{array}{l}
g(., \theta)=g\left(., \theta^{*}\right) \\
\theta, \theta^{*} \in \vartheta
\end{array} \Longrightarrow \theta=\theta^{*} .\right.
$$

This definition of identifiability is similar to the one in Grewal and Glover [1976]. A stochastic framework will be used for representing the uncertainty associated with data acquisition from the true system. The aforementioned signals, $\mathbf{e}(t)$ and $\mathbf{w}(t)$, will therefore be treated as stochastic processes. Consequently, an estimator 
will be of random nature and the quality of an estimator will have to be assessed in a statistical setting. The properties that are used to compare estimators are often asymptotic in the number of data points. One such property is consistency. An estimator of a parameter vector, $\theta$, is said to be consistent if it converges almost surely to the true value of the parameter vector.

Definition 2.2 (Consistency). An estimator of a parameter vector $\theta$ is consistent if

$$
\hat{\theta}_{N} \rightarrow \theta_{0} \text {, w.p. } 1 \text { as } N \rightarrow \infty \text {. }
$$

\subsubsection{Prediction error methods}

One of the most common ways of forming the value function in (2.9) is

$$
V_{N}(\mathcal{D}(N), \theta)=\frac{1}{N} \sum_{k=1}^{N} \ell(\mathbf{y}(k)-\hat{\mathbf{y}}(k \mid \theta)),
$$

where $\ell$ (.) is a scalar-valued function. An estimator that utilizes a value function like (2.12) is called a prediction error method (PEM). The parameters are then determined by solving

$$
\hat{\theta}_{N}^{\mathrm{PEM}}=\underset{\theta \in \vartheta}{\operatorname{argmin}} \frac{1}{N} \sum_{k=1}^{N} \ell(\mathbf{y}(k)-\hat{\mathbf{y}}(k \mid \theta)) .
$$

In general, this problem is not convex but simplifies for some model structures under specific choices of $\ell$ (.). One such scenario is when the predictor, (2.8), can be expressed as a linear function in the parameters

$$
\hat{\mathbf{y}}(k \mid \theta)=\Phi^{T}(\mathcal{D}(k-1)) \theta .
$$

Here $\Phi($.$) is called the regression matrix and its elements are known provided$ the data. Subsequently, the data dependence of $\Phi(k)=\Phi(\mathcal{D}(k-1))$ will for simplified notation not be written out explicitly. When the predictor is linear in the parameters, the residual

$$
\varepsilon(k \mid \theta)=\mathbf{y}(k)-\hat{\mathbf{y}}(k \mid \theta),
$$

is affine in the parameters. Then (2.13) will be a convex optimization problem if $\ell($.) is a convex function and $\vartheta$ is a convex set. In the special case where $\ell($.$) is$ a quadratic function, $\ell(\mathbf{x})=\mathbf{x}^{T} \mathbf{x}$, and $\vartheta$ is the set of real numbers, the estimator (2.13) can be expressed as an ordinary least-squares (LS) problem

$$
\hat{\theta}_{N}^{L S}=\underset{\theta}{\operatorname{argmin}} \frac{1}{N} \sum_{k=1}^{N}\left\|\mathbf{y}(k)-\Phi^{T}(k) \theta\right\|_{2}^{2} .
$$


The analytical solution to this optimization problem is

$$
\hat{\theta}_{N}^{L S}=\left(\frac{1}{N} \sum_{k=1}^{N} \Phi(k) \Phi^{T}(k)\right)^{-1}\left(\frac{1}{N} \sum_{k=1}^{N} \Phi(k) \mathbf{y}(k)\right),
$$

provided that the indicated inverse exists. In this work it will be assumed that the data is sufficiently informative and that the model structure is globally identifiable. In that case the inverse does exist.

\subsubsection{Instrumental variable methods}

The instrumental variable (IV) method constitutes an alternative to PEM. An IV estimator can be defined as an optimization problem similar to earlier or as the solution to a system of algebraic equations

$$
\hat{\theta}_{N}^{I V}=\operatorname{sol}\left\{\frac{1}{N} \sum_{k=1}^{N} \mathbf{Z}(k)\left(\mathbf{y}(k)-\Phi^{T}(k) \theta\right)=0\right\} .
$$

Here $\mathbf{Z}(k)$ is called the instrument matrix and the notation $\operatorname{sol}\{f(\mathbf{x})=0\}$ is used for the solution to the system of equations $f_{i}(\mathbf{x})=0, i=1, \ldots, n$. The IV estimator will be consistent if

$$
\begin{array}{r}
\bar{E}\left\{\mathbf{Z}(k) \Phi^{T}(k)\right\} \text { is full rank, } \\
\bar{E}\left\{\mathbf{Z}(k)\left(\mathbf{y}(k)-\Phi^{T}(k) \theta_{0}\right)\right\}=0,
\end{array}
$$

where the notation $\bar{E}\{\}=.\lim _{N \rightarrow \infty} \frac{1}{N} \sum_{k=1}^{N} E\{$.$\} was adopted from Ljung [1999].$ In general, if (2.19a) is fulfilled the parameters of an IV estimator will converge to the values that make

$$
\bar{E}\left\{\mathbf{Z}(k)\left(\mathbf{y}(k)-\Phi^{T}(k) \theta\right)\right\}=0 .
$$

This equation will be important for the analysis in Chapter 5 and will subsequently be referred to as the IV equation.

The analytical solution to $(2.18)$ is

$$
\hat{\theta}_{N}^{I V}=\left(\frac{1}{N} \sum_{k=1}^{N} \mathbf{Z}(k) \Phi^{T}(k)\right)^{-1}\left(\frac{1}{N} \sum_{k=1}^{N} \mathbf{Z}(k) \mathbf{y}(k)\right),
$$

provided that the indicated inverse exists. By comparing (2.17) with (2.21), the LS estimator can be seen to be a special case of the IV estimator, with $\mathbf{Z}(k)=\Phi(k)$. It is easy to find examples where an LS estimator is not consistent and the flexibility to choose $\mathbf{Z}(k)$ gives increased chances of obtaining consistency. On the other hand, the variance properties of an IV estimator depend highly on the choice of instruments and might very well be worse than those of a LS estimator. See for example Söderström and Stoica [1989] for more details about IV methods. 


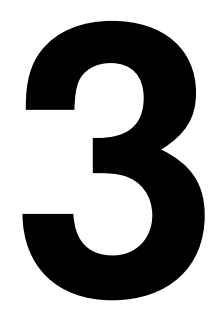

\section{Marine Modelling}

Different parametric model structures for ship dynamics have been proposed in the past. The aim of this work is not to develop new theory in that regard. Instead, focus is on developing parameter estimators, for a fairly general class of nonlinear models for marine vessels called second-order modulus models. This chapter serves as an introduction to ship modelling and contains no new results. The presented theory is based on the ideas found in for instance, Fossen [1994], Perez [2005] and Fossen [2011]. For simplified notation, the time dependence of continuous-time signals will in this chapter not be written out explicitly.

\subsection{The undisturbed equations of motion}

For describing motion of ships and other marine vehicles operating in multiple degrees of freedom (DOF), it is convenient to first define two coordinate systems.

Definition 3.1 ( $b$-frame). The $b$-frame has its origin, $O_{b}$, in the ship. The $x_{b}$ axis points towards the bow, i.e. forward, whereas the $y_{b}$-axis points starboard (right) and the $z_{b}$-axis points downwards. See Figure 3.1 for an illustration.

Definition 3.2 ( $n$-frame). The $n$-frame has its origin, $O_{n}$, fixed to earth. The $x_{n}$-axis points towards the north, whereas the $y_{n}$-axis points east and the $z_{n}$-axis points downwards, see Figure 3.1. This is sometimes referred to as a north-eastdown (NED) system.

For ships moving in wide areas, an Earth-centered reference frame can also be considered. However, for the analysis in this thesis, the above defined coordinate systems will be sufficient. 


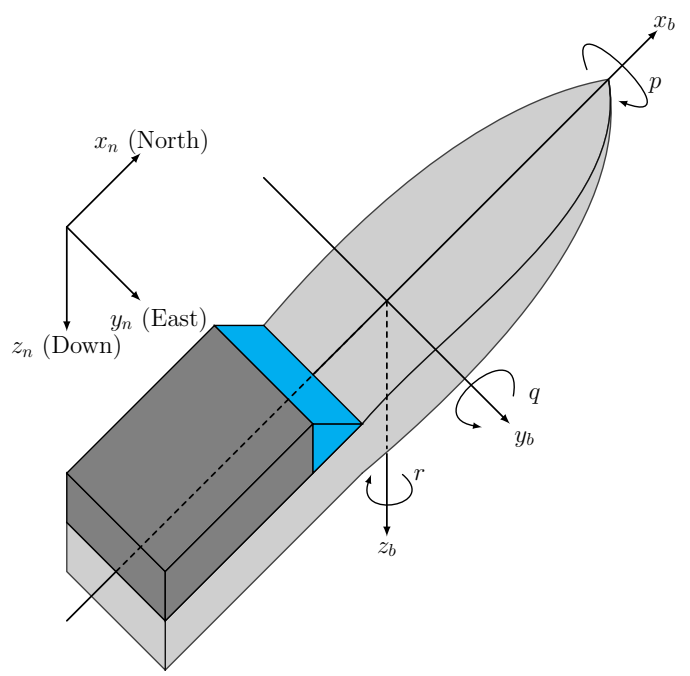

Figure 3.1: Illustration of a ship showing the reference frames in Definitions 3.1 and 3.2.

The equations of motion under undisturbed conditions are in Fossen [2011] formulated as

$$
\begin{aligned}
\dot{\eta} & =J(\eta) v, \\
M \dot{v}+C(v) v+D(v) v+g(\eta)+g_{0} & =\tau_{\mathrm{act}} .
\end{aligned}
$$

Here the first state vector

$$
\eta=\left[\begin{array}{llllll}
x_{n} & y_{n} & z_{n} & \phi & \theta & \psi
\end{array}\right]^{T},
$$

constitutes global position and attitude in the form of Euler angles between the $n$-frame and the $b$-frame. The second state vector

$$
v=\left[\begin{array}{llllll}
u & v & w & p & q & r
\end{array}\right]^{T},
$$

includes the translational velocities in the $b$-frame and angular velocities about the $b$-frame axes, as in Figure 3.1. The translational velocities, $u=\dot{x}_{b}, v=\dot{y}_{b}, w=$ $\dot{z}_{b}$ are by convention referred to as surge, sway and heave, respectively, and the Euler angles, $\phi, \theta$ and $\psi$ are for the same reason referred to as roll, pitch and yaw. Notably, the order of the Euler angles is important and the $z y x$ convention is used. This determines the structure of $J(\eta)$, which is an attitude dependent rotation matrix. Further, $M$ is a matrix including mass and inertia elements in accordance with Newton's laws, $C(v)$ includes Coriolis and centripetal forces that are due to the rotation of the $b$-frame about the $n$-frame, $D(v)$ describes energy losses due to damping and $g(\eta)+g_{0}$ are static forces, for example caused by buoyancy and gravity. The system input $\tau_{\text {act }}$ is a collection of forces and moments caused by the 
ship's actuators. Note that the model (3.1) can be nonlinear despite the matrix representation, because some matrices are velocity dependent and some matrices depend on the ship's orientation.

Any motion of a marine craft will induce motion in the surrounding water. It is therefore common to divide the mass and inertia matrix, $M$, as well as the Coriolis matrix, $C(v)$, into separate parts for rigid-body kinetics, which comprises forces and moments caused by moving the vessel itself and hydrodynamics, which comprises forces and moments caused by the moved water

$$
\begin{aligned}
M & =M_{R B}+M_{A}, \\
C(v) & =C_{R B}(v)+C_{A}(v) .
\end{aligned}
$$

From a system identification point of view this separation is unappealing, because unique identification of the two effects is not necessarily possible. It would therefore be desirable to maintain the compact notion of (3.1b). The separation is however required for a correct treatment of environmental disturbances.

\subsection{Environmental disturbances}

In addition to the ship's actuators, there are mainly three sources of environmental disturbances that affect the vessel. These are ocean currents, wind and waves. In this thesis, only ocean currents and wind are dealt with.

When the surrounding water is not at stand-still with respect to the inertial frame, which is the case if ocean currents are considered, the rigid-body effects enter the system in a different way than the hydrodynamic effects do. Denoting the generalized velocity vector of the water in the body-fixed frame as $v_{c}$ and the corresponding relative velocity of the ship as $v_{r}=v-v_{c}$, the rigid-body effects depend on the ship's absolute velocity

$$
\tau_{R B}=M_{R B} \dot{v}+C_{R B}(v) v,
$$

whereas the hydrodynamic forces and moments depend on the relative velocity between the ship and the surrounding water

$$
\tau_{\mathrm{hyd}}=M_{A} \dot{v}_{r}+C_{A}\left(v_{r}\right) v_{r}+D\left(v_{r}\right) v_{r}
$$

Regarding wind disturbances it is common to assume the principles of superposition and neglect the fact that the aerodynamic forces depend on the velocity of the ship. This is a reasonable approximation in some cases but in general the wind effect will be nonlinear and enter system both additively and multiplicatively in the equations of motion. To be able to develop consistent estimators in the later chapters of this thesis, a more accurate description of the wind forces will be needed. Therefore, denote the generalized velocity vector of the surrounding air in the body-fixed frame as $v_{w}$ and the corresponding relative velocity of the ship as $v_{q}=v-v_{w}$. The aerodynamic damping matrix $F\left(v_{q}\right)$ will be used to 
describe effects of the wind as well as regular air resistance. In theory, there will be both added-mass and Coriolis effects connected to the moved air as well but these are supposedly small and a damping matrix will be assumed sufficient for capturing all relevant aerodynamic effects

$$
\tau_{\text {air }}=F\left(v_{q}\right) v_{q}
$$

The equations of motion including environmental disturbances can now be written as

$$
M_{R B} \dot{v}+M_{A} \dot{v}_{r}+C_{R B}(v) v+C_{A}\left(v_{r}\right) v_{r}+D\left(v_{r}\right) v_{r}+F\left(v_{q}\right) v_{q}+g(\eta)+g_{0}=\tau_{\mathrm{act}} .
$$

The ocean and air currents are modelled as stationary stochastic processes in the inertial frame

$$
\begin{gathered}
v_{c, n} \sim P_{v_{c, n}}, \\
v_{w, n} \sim P_{v_{w, n}} .
\end{gathered}
$$

In the body-fixed frame, they depend on the attitude of the ship

$$
\begin{gathered}
v_{c}=J^{-1}(\eta) v_{c, n}, \\
v_{w}=J^{-1}(\eta) v_{w, n} .
\end{gathered}
$$

It will be assumed that the ocean current is almost constant in the $n$-frame, $\dot{v}_{c, n} \approx$ 0 and that the ship is turning slowly so that the acceleration of the current is negligible also in the $b$-frame, $\dot{v}_{c} \approx 0$. In this case, the added-mass term is

$$
M_{A} \dot{v}_{r}=M_{A}\left(\dot{v}-\dot{v}_{c}\right) \approx M_{A} \dot{v} .
$$

Having to assume that the ship is turning slowly is a bit restricting but it greatly simplifies the parameter estimation discussed in the forthcoming chapters. If (3.14) holds and if $M=M_{R B}+M_{A}$ is nonsingular, (3.9) can be cast on state-space form as

$$
\dot{v}=M^{-1}\left(-C_{R B}(v) v-\left(C_{A}\left(v_{r}\right)+D\left(v_{r}\right)\right) v_{r}-F\left(v_{q}\right) v_{q}+\tau_{\mathrm{act}}-g(\eta)-g_{0}\right) .
$$

\subsection{Maneuvering models}

Three DOF maneuvering models constitute an important special case of the general model described above. In this case, only motion in the horizontal plane is considered and the state vectors, with some abuse of notation, are

$$
\begin{aligned}
& v=[u, v, r]^{T}, \\
& \eta=\left[x_{n}, y_{n}, \psi\right]^{T} .
\end{aligned}
$$


This means that the dynamics associated with motion in heave, roll and pitch are neglected, $w=p=q=0$. For horizontal motion of a vessel the kinematic equations, $(3.1 \mathrm{a})$, reduce to one principal rotation about the $z_{b}$-axis

$$
J_{\Theta}(\eta)=R(\psi)=\left[\begin{array}{ccc}
\cos (\psi) & -\sin (\psi) & 0 \\
\sin (\psi) & \cos (\psi) & 0 \\
0 & 0 & 1
\end{array}\right] \text {. }
$$

In maneuvering theory, it is also common to neglect the static forces $g(\eta)$ and $g_{0}$. This means that the state-space representation (3.15) simplifies as

$$
\dot{v}=M^{-1}\left(-C_{R B}(v) v-\left(C_{A}\left(v_{r}\right)+D\left(v_{r}\right)\right) v_{r}-F\left(v_{q}\right) v_{q}+\tau_{\text {act }}\right) \text {. }
$$

\subsection{Nonlinear ship modelling}

The model (3.19) is nonlinear due to the velocity dependence of $C_{R B}(v), C_{A}\left(v_{r}\right)$, $D\left(v_{r}\right)$ and $F\left(v_{q}\right)$. There are many possible representations of the Coriolis and centripetal matrices but in general only first-order terms are present, meaning that the resulting expressions, $C_{R B}(v) v$ and $C_{A}\left(v_{r}\right) v_{r}$, only include terms of second order. The most uncertain component in the model is perhaps the damping vector, $D\left(v_{r}\right) v_{r}$, to which many hydrodynamic phenomena contribute. Based on knowledge from fluid dynamics it is often expanded in a series in one of two ways.

\subsubsection{Truncated Taylor expansion}

The first approach is to base the series on a Taylor expansion and this was suggested by Abkowitz [1964]. If Taylor expansions are considered, the even-order terms are usually neglected. This is done in order to enforce that the resulting model behaves in the same way for positive and negative relative velocities, something that is necessary due to ship symmetry.

\subsubsection{Second-order modulus models}

The other type of series expansion was first proposed by Fedyaevsky and Sobolev [1964]. Their suggestion is based on a combination of physical effects such as circulation and cross-flow drag principles, properties that are usually well-described by quadratic functions. The constraint of having a symmetric model is therefore instead resolved by use of the modulus function. These models are not necessarily continuously differentiable, and strictly speaking they can therefore not represent the physical system. However, experience has shown that they can describe the water's damping effects quite accurately and they are therefore often used anyway. Expansions of this type typically do not include any terms of higher 
order than two and the resulting models are therefore often referred to as secondorder modulus models.

For describing a general second-order modulus model, it is convenient to first define a second-order modulus function.

Definition 3.3. A second-order modulus function is a function, $f: \mathbb{R}^{n+p} \rightarrow \mathbb{R}^{m}$ that can be written as

$$
f(\mathbf{x}, \theta)=\Phi^{T}(\mathbf{x}) \theta,
$$

where each element of the $p \times m$ matrix $\Phi(\mathbf{x})$ is on one of the forms $x_{i},\left|x_{i}\right|, x_{i} x_{j}$, $x_{i}\left|x_{j}\right|$ for $i, j \leq n$ or zero and $\theta \in \mathbb{R}^{p}$ is a vector of coefficients.

It can be noted that by Definition 3.3, the sum of two second-order modulus functions can be expressed as a second-order modulus function

$$
\begin{aligned}
f_{1}\left(\mathbf{x}_{1}, \theta_{1}\right)+f_{2}\left(\mathbf{x}_{2}, \theta_{2}\right) & =\Phi_{1}^{T}\left(\mathbf{x}_{1}\right) \theta_{1}+\Phi_{2}^{T}\left(\mathbf{x}_{2}\right) \theta_{2}=\left[\begin{array}{l}
\Phi_{1}^{T}\left(\mathbf{x}_{1}\right) \Phi_{2}^{T}\left(\mathbf{x}_{2}\right)
\end{array}\right]\left[\begin{array}{l}
\theta_{1} \\
\theta_{2}
\end{array}\right] \\
& \triangleq \tilde{\Phi}^{T}\left(\left[\begin{array}{l}
\mathbf{x}_{1} \\
\mathbf{x}_{2}
\end{array}\right]\right)\left[\begin{array}{l}
\theta_{1} \\
\theta_{2}
\end{array}\right]=\tilde{f}\left(\left[\begin{array}{l}
\mathbf{x}_{1} \\
\mathbf{x}_{2}
\end{array}\right],\left[\begin{array}{l}
\theta_{1} \\
\theta_{2}
\end{array}\right]\right),
\end{aligned}
$$

and that the linear relationship $f(\mathbf{x})=A \mathbf{x}$, is a special case of a second-order modulus function.

In this work, the second-order modulus approach will be adopted. This means that the hydrodynamic damping matrix, $D\left(v_{r}\right)$, and its aerodynamic counterpart, $F\left(v_{q}\right)$, will be assumed to include elements of first order, possibly with absolute values. Together with the aforementioned assumption that $C_{R B}(v)$ and $C_{A}\left(v_{r}\right)$ only include elements of first order, each individual term on the right-hand side of (3.19) can be viewed as a second-order modulus function. Consequently, the whole equation can be viewed as a second-order modulus function

$$
\begin{aligned}
\dot{v} & =M^{-1}\left(-C_{R B}(v) v-\left(C_{A}\left(v_{r}\right)+D\left(v_{r}\right)\right) v_{r}-F\left(v_{q}\right) v_{q}+\tau_{\text {act }}\right) \\
& \triangleq f_{v}\left(v, \theta_{v}\right)+f_{v_{r}}\left(v_{r}, \theta_{v_{r}}\right)+f_{v_{q}}\left(v_{q}, \theta_{v_{q}}\right)+f_{\tau}\left(\tau_{\mathrm{act}}, \theta_{\tau}\right)=f\left(\left[\begin{array}{c}
v \\
v_{r} \\
v_{q} \\
\tau_{\mathrm{act}}
\end{array}\right],\left[\begin{array}{c}
\theta_{C} \\
\theta_{D} \\
\theta_{F} \\
\theta_{\tau}
\end{array}\right]\right) .
\end{aligned}
$$

Here $\theta_{v}, \theta_{v_{r}}, \theta_{v_{q}}$ and $\theta_{\tau}$ are time-independent parameters connected to the matrices $M, C_{R B}, C_{A}, D$ and $F$.

\subsection{Deriving a model structure}

For presenting the theory in Chapter 5, the simulations in Chapter 6 and the analysis of real data in Chapter 7, a specific ship model will be used. In the subsequent sections, that model is presented. 


\subsubsection{Rigid-body kinetics}

The effects of forces acting on a rigid body are called kinetics and can, for example, be derived based on Newton's laws. Here, the derivation is omitted and the interested reader is referred to Fossen [1994] or Fossen [2011]. One way to express the rigid-body mass matrix for maneuvering models is

$$
M_{R B}=\left[\begin{array}{ccc}
m & 0 & 0 \\
0 & m & m x_{G} \\
0 & m x_{G} & I_{z}
\end{array}\right],
$$

where $m$ is the mass of the ship, $x_{G}$ is the distance between the center of gravity and the origin $O_{b}$ and $I_{z}$ is the moment of inertia about the $z_{b}$-axis. If the distance between the center of gravity and $O_{b}$ is small, the diagonal approximation

$$
M_{R B} \approx\left[\begin{array}{ccc}
m & 0 & 0 \\
0 & m & 0 \\
0 & 0 & I_{z}
\end{array}\right]
$$

will be good. This was the case for the ship studied in Chapter 7 and therefore the diagonal structure will be used.

There are many possible representations of the Coriolis and centripetal matrix. In Fossen [2011], the representation

$$
C_{R B}(v)=\left[\begin{array}{ccc}
0 & 0 & -m\left(x_{G} r+v\right) \\
0 & 0 & m u \\
m\left(x_{G} r+v\right) & -m u & 0
\end{array}\right],
$$

was suggested. Making the same assumption as above of having $x_{G} \approx 0$, gives the slightly simplified expression

$$
C_{R B}(v) \approx\left[\begin{array}{ccc}
0 & 0 & -m v \\
0 & 0 & m u \\
m v & -m u & 0
\end{array}\right] .
$$

If the ocean current is irrotational

$$
v_{c}=\left[\begin{array}{lll}
u_{c} & v_{c} & 0
\end{array}\right]^{T},
$$

slowly changing in the $n$-frame, $\dot{v}_{c, n} \approx 0$, and $C_{R B}$ is parameterized independently of linear velocities, Hegrenæs [2010] has shown that

$$
M_{R B} \dot{v}+C_{R B}(v) v=M_{R B} \dot{v}_{r}+C_{R B}\left(v_{r}\right) v_{r} .
$$

It can be noted that the choice (3.25) gives the generalized force vector

$$
C_{R B}(v) v=\left[\begin{array}{ccc}
0 & 0 & -m v \\
0 & 0 & m u \\
m v & -m u & 0
\end{array}\right]\left[\begin{array}{l}
u \\
v \\
r
\end{array}\right]=\left[\begin{array}{c}
-m v r \\
m u r \\
0
\end{array}\right],
$$


and that an alternative representation that gives exactly the same forces is

$$
\tilde{C}_{R B}(v)=\left[\begin{array}{ccc}
0 & -m r & 0 \\
m r & 0 & 0 \\
0 & 0 & 0
\end{array}\right] .
$$

Here, the matrix is parameterized independently of linear velocities which means that the property (3.27) can be utilized.

\subsubsection{Hydrodynamics}

The added mass matrix will also be assumed to be diagonal

$$
M_{A}=\left[\begin{array}{ccc}
-X_{\dot{u}} & 0 & 0 \\
0 & -Y_{\dot{v}} & 0 \\
0 & 0 & -N_{\dot{r}}
\end{array}\right] .
$$

The parameters $X_{\dot{u}}, Y_{\dot{v}}$ and $N_{\dot{r}}$ are referred to as hydrodynamic derivatives and the notation is adopted from SNAME [1950]. For example the hydrodynamic added mass force $X$ along the $x_{b}$ axis due to an acceleration $\dot{u}$ in the $x_{b}$-direction is written as

$$
X=X_{\dot{u}} \dot{u},
$$

where $X_{\dot{u}}=\frac{\partial X}{\partial \dot{u}}$.

The hydrodynamic Coriolis and centripetal matrix is assumed to have the same structure as its rigid-body counterpart and is given by

$$
C_{A}\left(v_{r}\right)=\left[\begin{array}{ccc}
0 & 0 & Y_{\dot{v}} v_{r} \\
0 & 0 & -X_{\dot{u}} u_{r} \\
-Y_{\dot{v}} v_{r} & X_{\dot{u}} u_{r} & 0
\end{array}\right] .
$$

The expression for the hydrodynamic damping is adopted from Blanke [1981]. He suggested a simplified version of what was originally proposed by Norrbin [1970], namely that

$$
\tilde{D}\left(v_{r}\right)=\left[\begin{array}{ccc}
-X_{|u| u}\left|u_{r}\right| & 0 & 0 \\
0 & -Y_{|v| v}\left|v_{r}\right| & -Y_{|v| r}\left|v_{r}\right| \\
0 & -N_{|v| v}\left|v_{r}\right| & -N_{|v| r \mid}\left|v_{r}\right|
\end{array}\right] .
$$

This damping matrix will be supplemented with viscous friction

$$
\bar{D}=\left[\begin{array}{ccc}
-X_{u} & 0 & 0 \\
0 & -Y_{v} & 0 \\
0 & 0 & -N_{r}
\end{array}\right],
$$


such that

$$
D\left(v_{r}\right)=\bar{D}+\tilde{D}\left(v_{r}\right)=\left[\begin{array}{ccc}
-X_{u}-X_{|u| u}\left|u_{r}\right| & 0 & 0 \\
0 & -Y_{v}-Y_{|v| v}\left|v_{r}\right| & -Y_{|v| r}\left|v_{r}\right| \\
0 & -N_{|v| v \mid}\left|v_{r}\right| & -N_{r}-N_{|v| r}\left|v_{r}\right|
\end{array}\right] .
$$

Quadratic damping dominates at high speeds whereas viscous damping is more prominent at lower speeds. Having linear friction terms in the model therefore gives increased possibility of adaption to data collected when the ship is moving slowly.

\subsubsection{Aerodynamics}

The aerodynamic forces and moments are significantly smaller than the hydrodynamic forces and moments. Consequently, the aerodynamic part of the model is harder to identify. The simple representation

$$
F\left(v_{q}\right)=\left[\begin{array}{ccc}
-W_{|u| u \mid}\left|u_{q}\right| & 0 & 0 \\
0 & -W_{|v| v}\left|v_{q}\right| & 0 \\
0 & -W_{u v} u_{q} & 0
\end{array}\right],
$$

without viscous friction, will therefore be used in order to keep the number of unknown parameters low. This representation gives expressions of wind forces and moments that are similar to the ones given in Fossen [2011]. Unlike the expressions suggested by Fossen, (3.34) however gives wind forces and moments on second-order modulus form. This is convenient for the subsequent analysis in this thesis because it enables a unified treatment of disturbances caused by ocean currents and wind.

\subsubsection{Azimuth actuation}

Historically, the actuator force and moment components in the vector $\tau_{\text {act }}$, have originated from tunnel thrusters and rudders. These types of excitation are thoroughly covered in the aforementioned references. A more recently advanced technology is azimuth actuation. This is a type of thruster which it is possible to rotate about a vertical axis.

Assume that $N_{a}$ azimuth thrusters are placed on the ship's hull. Let azimuth thruster $i$ be running with a propeller speed $n_{i}$ at a variable angle $\alpha_{i}$. Further, assume that $\alpha_{i}=0$ when the azimuth thruster is pointing towards the bow of the ship (forward) and that $\alpha_{i}$ increases with negative rotation about the $z_{b}$-axis. Annotate the force in $x_{b}$-direction originating from azimuth thruster $i$ as

$$
F_{x, i}=g_{x}\left(n_{i}, \alpha_{i}, u_{a, i}\right) .
$$

Here $u_{a, i}$ is the advance velocity, i.e. the speed of the water passing thruster $i$ in negative $x_{b}$-direction. The dependence of $u_{a, i}$ is needed because the propeller 


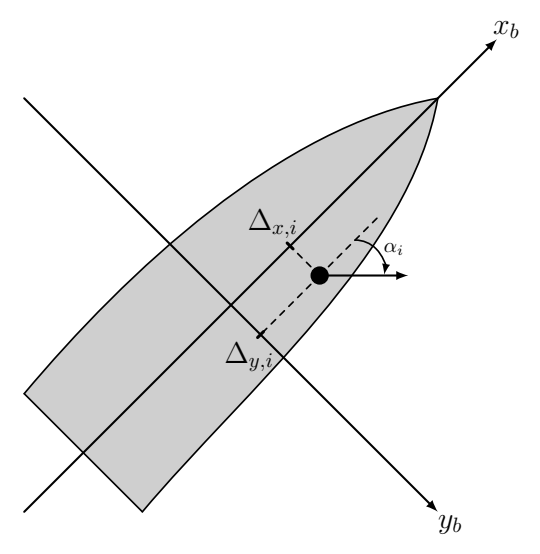

Figure 3.2: The rotation and mounting position of azimuth $i$, which is marked as a black dot.

efficiency falls off in the directional region of operation where the ship has substantial speed, i.e. when $u_{a, i}$ and $n_{i} \cos \left(\alpha_{i}\right)$ are of the same sign and $u_{a, i}$ increases in magnitude. Furthermore, some propellers show linear behavior in propeller speed while others show a quadratic one. Here it will be assumed that $g_{x}($.$) is$ linear in $n_{i}$ and consequently that the force can be expressed as

$$
g_{x}\left(n_{i}, \alpha_{i}, u_{a, i}\right)=\mu_{i} n_{i} \cos \left(\alpha_{i}\right)-\kappa_{i}^{\prime} n_{i} u_{a, i} \cos \left(\alpha_{i}\right),
$$

where $\mu_{i}$ and $\kappa_{i}^{\prime}$ are positive constants. Moreover, it will be assumed that

$$
u_{a, i}=\left(1-w_{i}\right) u_{r}
$$

where the wake fraction number, $w_{i} \in[0,1]$, determines the difference between the ship's speed and the flow velocity over the propeller disc. Following this, (3.36) can instead be expressed as

$$
\begin{aligned}
g_{x}\left(n_{i}, \alpha_{i}, u_{a, i}\right) & =\mu_{i} n_{i} \cos \left(\alpha_{i}\right)-\kappa_{i}^{\prime} n_{i} u_{r}\left(1-w_{i}\right) \cos \left(\alpha_{i}\right) \\
& =\mu_{i} n_{i} \cos \left(\alpha_{i}\right)-\kappa_{i} n_{i} u_{r} \cos \left(\alpha_{i}\right),
\end{aligned}
$$

where the last equality follows by introducing another positive constant $\kappa_{i}=$ $\kappa_{i}^{\prime}\left(1-w_{i}\right)$. The function $g_{x}($.$) is in reality more complex, for example, due to hull$ effects and because other actuators affect the force as a result of cross streams. However, the model (3.38) will be sufficient for the analysis in this work.

Following similar reasoning as above, it will be assumed that the force in $y_{b}$ direction originating from azimuth $i$ is

$$
\begin{aligned}
F_{y, i}=g_{y}\left(n_{i}, \alpha_{i}, v_{a, i}\right) & =\mu_{i} n_{i} \sin \left(\alpha_{i}\right)-\kappa_{i}^{\prime} n_{i} v_{a, i} \sin \left(\alpha_{i}\right) \\
& =\mu_{i} n_{i} \sin \left(\alpha_{i}\right)-\kappa_{i} n_{i} v_{r} \sin \left(\alpha_{i}\right)
\end{aligned}
$$


Azimuth thruster $i$ will also generate a torque and this torque will depend on where the azimuth is mounted. Assuming that azimuth $i$ is mounted at $\left(\Delta_{x, i}, \Delta_{y, i}\right)$ in the $b$-frame, as in Figure 3.2, the resulting torque about the $z_{b}$-axis is

$$
M_{i}=\Delta_{x, i} F_{y, i}-\Delta_{y, i} F_{x, i}
$$

The generalized force vector consists of the sum of the forces and moments from all the azimuth thrusters combined

$$
\tau_{\mathrm{act}}=\left[\begin{array}{c}
\sum_{i=1}^{N_{a}} \mu_{i} n_{i} \cos \left(\alpha_{i}\right)-\kappa_{i} n_{i} u_{r} \cos \left(\alpha_{i}\right) \\
\sum_{i=1}^{N_{a}} \mu_{i} n_{i} \sin \left(\alpha_{i}\right)-\kappa_{i} n_{i} v_{r} \sin \left(\alpha_{i}\right) \\
\sum_{i=1}^{N_{a}} n_{i}\left[\mu_{i}\left(\Delta_{x, i} \sin \left(\alpha_{i}\right)-\Delta_{y, i} \cos \left(\alpha_{i}\right)\right)-\kappa_{i}\left(\Delta_{x, i} v_{r} \sin \left(\alpha_{i}\right)-\Delta_{y, i} u_{r} \cos \left(\alpha_{i}\right)\right)\right]
\end{array}\right] .
$$

In order to keep the model complexity low, the dependence on the advance velocity will only be considered for the force in $x_{b}$-direction where the corresponding effect is most prominent

$$
\tau_{\mathrm{act}} \approx\left[\begin{array}{c}
\sum_{i=1}^{N_{a}} \mu_{i} n_{i} \cos \left(\alpha_{i}\right)-\kappa_{i} n_{i} u_{r} \cos \left(\alpha_{i}\right) \\
\sum_{i=1}^{N_{a}} \mu_{i} n_{i} \sin \left(\alpha_{i}\right) \\
\sum_{i=1}^{N_{a}} \mu_{i} n_{i}\left(\Delta_{x, i} \sin \left(\alpha_{i}\right)-\Delta_{y, i} \cos \left(\alpha_{i}\right)\right)
\end{array}\right]
$$

For the same reason it will be assumed that all the azimuths are equally efficient

$$
\begin{aligned}
& \mu_{i}=\mu_{j} \triangleq \mu \forall i, j=1, \ldots, N_{a}, \\
& \kappa_{i}=\kappa_{j} \triangleq \kappa \forall i, j=1, \ldots, N_{a},
\end{aligned}
$$

so that the generalized torque vector can be expressed as

$$
\tau_{\mathrm{act}}=\left[\begin{array}{c}
\mu \tilde{\tau}_{x} \\
\mu \tilde{\tau}_{y} \\
\mu \tilde{\tau}_{\psi}
\end{array}\right]+\left[\begin{array}{c}
\kappa u_{r} \tilde{\tau}_{x} \\
0 \\
0
\end{array}\right]
$$

where

$$
\tilde{\tau}=\left[\begin{array}{c}
\tilde{\tau}_{x} \\
\tilde{\tau}_{y} \\
\tilde{\tau}_{\psi}
\end{array}\right] \triangleq\left[\begin{array}{c}
\sum_{i=1}^{N_{a}} n_{i} \cos \left(\alpha_{i}\right) \\
\sum_{i=1}^{N_{a}} n_{i} \sin \left(\alpha_{i}\right) \\
\sum_{i=1}^{N_{a}} n_{i}\left(\Delta_{x, i} \sin \left(\alpha_{i}\right)-\Delta_{y, i} \cos \left(\alpha_{i}\right)\right)
\end{array}\right]
$$

is known if the system inputs, $n_{i}, \alpha_{i}$, as well as the azimuths' mounting positions, $\Delta_{x, i}, \Delta_{y, i}$, are known for all $i=1, \ldots, N_{A}$. Then, the parameters left to be estimated are $\mu$ and $\kappa$. 


\subsubsection{State-space representation}

By the property (3.27), it is possible to write the disturbed equations of motion in the horizontal plane as

$$
M \dot{v}_{r}+C\left(v_{r}\right) v_{r}+D\left(v_{r}\right) v_{r}+F\left(v_{q}\right) v_{q}=\tau_{\mathrm{act}},
$$

where $M=M_{R B}+M_{A}$ and $C\left(v_{r}\right)=C_{R B}\left(v_{r}\right)+C_{A}\left(v_{r}\right)$. The assumption $\dot{v}_{c} \approx 0$ gives, as in (3.14), that

$$
M \dot{v}_{r} \approx M \dot{v} .
$$

Then, using the proposed force and moment representations, (3.23), (3.29), (3.30), (3.32), (3.33), (3.34) and (3.45), a model on state-space form is given by

$$
\begin{aligned}
& \dot{v}=\left[\begin{array}{lll}
\dot{u} & \dot{v} & \dot{r}
\end{array}\right]^{T}=M^{-1}\left(-\left(C\left(v_{r}\right)+D\left(v_{r}\right)\right) v_{r}-F\left(v_{q}\right) v_{q}+\tau_{\text {act }}\right) \\
& =\left[\begin{array}{ccc}
\frac{1}{m-X_{\dot{u}}} & 0 & 0 \\
0 & \frac{1}{m-Y_{\dot{v}}} & 0 \\
0 & 0 & \frac{1}{I_{z}-N_{\dot{r}}}
\end{array}\right]\left(-\left[\begin{array}{c}
-m v_{r} r+Y_{\dot{v}} v_{r} r-X_{u} u_{r}-X_{|u| u}\left|u_{r}\right| u_{r} \\
m u_{r} r-X_{\dot{u}} u_{r} r-Y_{v} v_{r}-Y_{|v| v}\left|v_{r}\right| v_{r}-Y_{|v| r}\left|v_{r}\right| r \\
\left(X_{\dot{u}}-Y_{\dot{v}}\right) u_{r} v_{r}-N_{r} r-N_{|v| v \mid}\left|v_{r}\right| v_{r}-N_{|v| r}\left|v_{r}\right| r
\end{array}\right]\right. \\
& \left.-\left[\begin{array}{c}
-W_{|u| u}\left|u_{q}\right| u_{q} \\
-W_{|v| v}\left|v_{q}\right| v_{q} \\
-W_{u v} u_{q} v_{q}
\end{array}\right]+\left[\begin{array}{c}
\left(\mu+\kappa u_{r}\right) \tilde{\tau}_{x} \\
\mu \tilde{\tau}_{y} \\
\mu \tilde{\tau}_{\psi}
\end{array}\right]\right) \\
& =\left[\begin{array}{c}
\frac{1}{m-X_{\dot{u}}}\left(\left(m-Y_{\dot{v}}\right) v_{r} r+X_{u} u_{r}+X_{|u| u}\left|u_{r}\right| u_{r}+W_{|u| u}\left|u_{q}\right| u_{q}+\mu \tilde{\tau}_{x}+\kappa u_{r} \tilde{\tau}_{x}\right) \\
\frac{1}{m-Y_{\dot{v}}}\left(-\left(m-X_{\dot{u}}\right) u_{r} r+Y_{v} v_{r}+Y_{|v| v}\left|v_{r}\right| v_{r}+Y_{|v| r}\left|v_{r}\right| r+W_{|v| v}\left|v_{q}\right| v_{q}+\mu \tilde{\tau}_{y}\right) \\
\frac{1}{I_{z}-N_{\dot{r}}}\left(-\left(X_{\dot{u}}-Y_{\dot{v}}\right) u_{r} v_{r}+N_{r} r+N_{|v| v}\left|v_{r}\right| v_{r}+N_{|v| r \mid}\left|v_{r}\right| r+W_{u v} u_{q} v_{q}+\mu \tilde{\tau}_{\psi}\right)
\end{array}\right] .
\end{aligned}
$$

Casting this as a discrete-time model using Euler's explicit method gives

$$
\begin{aligned}
u\left(k T_{s}+\right. & \left.T_{s}\right)=u\left(k T_{s}\right)+\frac{T_{s}}{m-X_{\dot{u}}}\left(\left(m-Y_{\dot{v}}\right) v_{r}\left(k T_{s}\right) r\left(k T_{s}\right)+X_{u} u_{r}\left(k T_{s}\right)\right. \\
& +X_{|u| u}\left|u_{r}\left(k T_{s}\right)\right| u_{r}\left(k T_{s}\right)+W_{|u| u}\left|u_{q}\left(k T_{s}\right)\right| u_{q}\left(k T_{s}\right)+\mu \tilde{\tau}_{x}\left(k T_{s}\right) \\
& \left.+\kappa u_{r}\left(k T_{s}\right) \tilde{\tau}_{x}\left(k T_{s}\right)\right), \\
v\left(k T_{s}+\right. & \left.T_{s}\right)=v\left(k T_{s}\right)+\frac{T_{s}}{m-Y_{\dot{v}}}\left(-\left(m+X_{\dot{u}}\right) u_{r}\left(k T_{s}\right) r\left(k T_{s}\right)+Y_{v} v_{r}\left(k T_{s}\right)\right. \\
& +Y_{|v| v \mid}\left|v_{r}\left(k T_{s}\right)\right| v_{r}\left(k T_{s}\right)+Y_{|v| r}\left|v_{r}\left(k T_{s}\right)\right| r\left(k T_{s}\right)+W_{|v| v}\left|v_{q}\left(k T_{s}\right)\right| v_{q}\left(k T_{s}\right) \\
& \left.+\mu \tilde{\tau}_{y}\left(k T_{s}\right)\right), \\
r\left(k T_{s}+\right. & \left.T_{s}\right)=r\left(k T_{s}\right)+\frac{T_{s}}{I_{z}-N_{\dot{r}}}\left(-\left(X_{\dot{u}}-Y_{\dot{v}}\right) u_{r}\left(k T_{s}\right) v_{r}\left(k T_{s}\right)+N_{r} r\left(k T_{s}\right)\right. \\
& +N_{|v| v}\left|v_{r}\left(k T_{s}\right)\right| v_{r}\left(k T_{s}\right)+N_{|v| r}\left|v_{r}\left(k T_{s}\right)\right| r\left(k T_{s}\right)+W_{u v} u_{q}\left(k T_{s}\right) v_{q}\left(k T_{s}\right) \\
& \left.+\mu \tilde{\tau}_{\psi}\left(k T_{s}\right)\right) .
\end{aligned}
$$


Redefining parameters in accordance with Table 3.1 makes it possible to express this model as a regression in the parameters, which under assumption of unit sampling time can be written as

$$
\left[\begin{array}{c}
u(k) \\
v(k) \\
r(k)
\end{array}\right]=\left[\begin{array}{c}
u(k-1) \\
v(k-1) \\
r(k-1)
\end{array}\right]+\left[\begin{array}{ccc}
\varphi_{u}^{T}(k) & 0 & 0 \\
0 & \varphi_{v}^{T}(k) & 0 \\
0 & 0 & \varphi_{r}^{T}(k)
\end{array}\right] \theta=\left[\begin{array}{c}
u(k-1) \\
v(k-1) \\
r(k-1)
\end{array}\right]+\Phi^{T}(k) \theta .
$$

Here

$$
\begin{gathered}
\varphi_{u}(k)=\left[\begin{array}{llll}
u_{r}(k-1) & v_{r}(k-1) r(k-1) & u_{r}(k-1)\left|u_{r}(k-1)\right| & \ldots \\
\ldots & u_{q}(k-1)\left|u_{q}(k-1)\right| & \tilde{\tau}_{x}(k-1) & u_{r}(k-1) \tilde{\tau}_{x}(k-1)
\end{array}\right]^{T}, \\
\varphi_{v}(k)=\left[\begin{array}{llll}
v_{r}(k-1) & u_{r}(k-1) r(k-1) & v_{r}(k-1)\left|v_{r}(k-1)\right| & \ldots \\
\ldots & r(k-1)\left|v_{r}(k-1)\right| & v_{q}(k-1)\left|v_{q}(k-1)\right| & \tilde{\tau}_{y}(k-1)
\end{array}\right]^{T}, \\
\varphi_{r}(k)=\left[\begin{array}{llll}
r(k-1) & u_{r}(k-1) v_{r}(k-1) & v_{r}(k-1)\left|v_{r}(k-1)\right| & \ldots \\
\ldots & r(k-1)\left|v_{r}(k-1)\right| & u_{q}(k-1) v_{q}(k-1) & \tilde{\tau}_{\psi}(k-1)
\end{array}\right]^{T},
\end{gathered}
$$

and

$$
\begin{aligned}
& \theta=\left[\begin{array}{llllllllll}
\mathcal{X}_{u} & \mathcal{X}_{v r} & \mathcal{X}_{|u| u} & \mathcal{W}_{|u| u} & \mathcal{X}_{\mu} & \mathcal{X}_{\mathcal{\kappa}} & \mathcal{Y}_{v} & \mathcal{Y}_{u r} & \mathcal{Y}_{|v| v} & \ldots
\end{array}\right. \\
& \begin{array}{lllllllllll} 
& \mathcal{Y}_{|v| r} & \mathcal{W}_{|v| v} & \mathcal{Y}_{\mu} & \mathcal{N}_{r} & \mathcal{N}_{u v} & \mathcal{N}_{|v| v} & \mathcal{N}_{|v| r} & \mathcal{W}_{u v} & \left.\mathcal{N}_{\mu}\right]^{T} .
\end{array}
\end{aligned}
$$

\subsubsection{Surge model}

If the motion in sway and yaw as well as the dependence of the advance speed in the thrust force are neglected, a simple surge model is obtained

$$
u(k+1)=u(k)+\mathcal{X}_{u} u_{r}(k)+\mathcal{X}_{|u| u}\left|u_{r}(k)\right| u_{r}(k)+\mathcal{W}_{|u| u}\left|u_{q}(k)\right| u_{q}(k)+\mathcal{X}_{\mu} \tilde{\tau}_{x}(k) .
$$

This model is sufficiently simple and transparent for analysis but yet the estimation of its parameters is a non-trivial task which includes most of the challenges discussed in this work. It is therefore used for presenting some of the theory in Chapter 5.

\subsection{Estimating velocity states}

A situation that sometimes occurs in practice is that only parts of the state vector can be measured directly. Easiest to measure are perhaps angular velocities and 
Table 3.1: Discrete-time aggregations of the model parameters.

\begin{tabular}{||c|c||}
\hline Discrete-time parameter & Continuous-time expression \\
$\mathcal{X}_{u}$ & $T_{s} \frac{X_{u}}{m-X_{\dot{u}}}$ \\
$\mathcal{X}_{v r}$ & $T_{s} \frac{m-Y_{\dot{v}}}{m-X_{\dot{u}}}$ \\
$\mathcal{X}_{|u| u}$ & $T_{s} \frac{X_{|u| u}}{m-X_{\dot{u}}}$ \\
$\mathcal{W}_{|u| u}$ & $T_{s} \frac{W_{|u| u}}{m-X_{\dot{u}}}$ \\
$\mathcal{X}_{\mu}$ & $T_{s} \frac{\mu}{m-X_{\dot{u}}}$ \\
$\mathcal{X}_{\mathcal{K}}$ & $T_{s} \frac{\kappa}{m-X_{\dot{u}}}$ \\
$\mathcal{Y}_{v}$ & $T_{s} \frac{Y_{v}}{m-Y_{\dot{v}}}$ \\
$\mathcal{Y}_{u r}$ & $-T_{s} \frac{m-X_{\dot{u}}}{m-Y_{\dot{v}}}$ \\
$\mathcal{Y}_{|v| v}$ & $T_{s} \frac{Y_{|v| v}}{m-Y_{\dot{v}}}$ \\
$\mathcal{Y}_{|v| r}$ & $T_{s} \frac{Y_{|v| r}}{m-Y_{\dot{v}}}$ \\
$\mathcal{W}_{|v| v}$ & $T_{s} \frac{W_{|v| v}}{m-Y_{\dot{v}}}$ \\
$\mathcal{Y}_{\mu}$ & $T_{s} \frac{\mu}{m-Y_{\dot{v}}}$ \\
$\mathcal{N}_{r}$ & $T_{s} \frac{N_{r}}{I_{z}-N_{\dot{r}}}$ \\
$\mathcal{N}_{u v}$ & $-T_{s} \frac{X_{\dot{u}}-Y_{\dot{v}}}{I_{z}-N_{\dot{r}}}$ \\
$\mathcal{N}_{|v| v}$ & $T_{s} \frac{N_{|v| v}}{I_{z}-N_{\dot{r}}}$ \\
$\mathcal{N}_{|v| r}$ & $T_{s} \frac{N_{|v| r}}{I_{z}-N_{\dot{r}}}$ \\
$\mathcal{W}_{u v}$ & $T_{s} \frac{W_{u v}}{I_{z}-N_{\dot{r}}}$ \\
$\mathcal{N}_{\mu}$ & $T_{s} \frac{\mu}{I_{z}-N_{\dot{r}}}$ \\
\hline & \\
\hline
\end{tabular}


accelerations using inertial sensors. Surface vessels are also often equipped with a global navigation satellite system (GNSS) receiver for positioning. However, the linear velocities are more difficult to measure directly. A simple way of obtaining an estimate of the velocity states, $\hat{v}$, based on measurements of the position and attitude states in the $n$-frame, $\mathbf{y}_{\eta}$, is a finite difference approximation

$$
\hat{v}(k)=\frac{1}{2 T_{s}} R\left(y_{\psi}(k)\right)\left(\mathbf{y}_{\eta}(k+1)-\mathbf{y}_{\eta}(k-1)\right) \text {. }
$$

A notable downside is however that this is not an unbiased estimator of the velocities if the yaw angle is measured with uncertainty. To see this, assume that $\eta(k)$ is measured with symmetrically distributed zero-mean white noise

$$
\mathbf{y}_{\eta}(k)=\left[\begin{array}{c}
y_{x_{n}}(k) \\
y_{y_{n}}(k) \\
y_{\psi}(k)
\end{array}\right]=\eta(k)+\mathbf{e}_{\eta}(k)=\left[\begin{array}{c}
x_{n}(k) \\
y_{n}(k) \\
\psi(k)
\end{array}\right]+\left[\begin{array}{c}
e_{x_{n}}(k) \\
e_{y_{n}}(k) \\
e_{\psi}(k)
\end{array}\right] .
$$

In this case

$$
\begin{aligned}
& \bar{E}\left\{R\left(y_{\psi}(k)\right)\left(\mathbf{y}_{\eta}(k+1)-\mathbf{y}_{\eta}(k-1)\right)\right\} \\
& =\bar{E}\left\{R\left(\psi(k)+e_{\psi}(k)\right) \cdot\left[\begin{array}{c}
x_{n}(k+1)-x_{n}(k-1)+e_{x_{n}}(k+1)-e_{x_{n}}(k-1) \\
y_{n}(k+1)-y_{n}(k-1)+e_{y_{n}}(k+1)-e_{y_{n}}(k-1) \\
\psi(k+1)-\psi(k-1)+e_{\psi}(k+1)-e_{\psi}(k-1)
\end{array}\right]\right\} \\
& =\bar{E}\left\{\left[\begin{array}{ccc}
\cos \left(\psi(k)+e_{\psi}(k)\right) & -\sin \left(\psi(k)+e_{\psi}(k)\right) & 0 \\
\sin \left(\psi(k)+e_{\psi}(k)\right) & \cos \left(\psi(k)+e_{\psi}(k)\right) & 0 \\
0 & 0 & 1
\end{array}\right]\right\} \cdot\left[\begin{array}{c}
x_{n}(k+1)-x_{n}(k-1) \\
y_{n}(k+1)-y_{n}(k-1) \\
\psi(k+1)-\psi(k-1)
\end{array}\right] \\
& +\bar{E}\left\{\left[\begin{array}{ccc}
\cos \left(\psi(k)+e_{\psi}(k)\right) & -\sin \left(\psi(k)+e_{\psi}(k)\right) & 0 \\
\sin \left(\psi(k)+e_{\psi}(k)\right) & \cos \left(\psi(k)+e_{\psi}(k)\right) & 0 \\
0 & 0 & 1
\end{array}\right] \cdot\left[\begin{array}{c}
e_{x_{n}}(k+1)-e_{x_{n}}(k-1) \\
e_{y_{n}}(k+1)-e_{y_{n}}(k-1) \\
e_{\psi}(k+1)-e_{\psi}(k-1)
\end{array}\right]\right\} \\
& =\left[\begin{array}{ccc}
\bar{E}\left\{\cos \left(\psi(k)+e_{\psi}(k)\right)\right\} & -\bar{E}\left\{\sin \left(\psi(k)+e_{\psi}(k)\right)\right\} & 0 \\
\bar{E}\left\{\sin \left(\psi(k)+e_{\psi}(k)\right)\right\} & \bar{E}\left\{\cos \left(\psi(k)+e_{\psi}(k)\right)\right\} & 0 \\
0 & 0 & 1
\end{array}\right] \cdot\left[\begin{array}{c}
x_{n}(k+1)-x_{n}(k-1) \\
y_{n}(k+1)-y_{n}(k-1) \\
\psi(k+1)-\psi(k-1)
\end{array}\right] .
\end{aligned}
$$

Further it holds that

$$
\begin{aligned}
& \bar{E}\left\{\sin \left(\psi(k)+e_{\psi}(k)\right)\right\}=\bar{E}\left\{\sin (\psi(k)) \cos \left(e_{\psi}(k)\right)+\cos (\psi(k)) \sin \left(e_{\psi}(k)\right)\right\} \\
& \quad=\sin (\psi(k)) \bar{E}\left\{\cos \left(e_{\psi}(k)\right)\right\}+\cos (\psi(k)) \bar{E}\left\{\sin \left(e_{\psi}(k)\right)\right\} \\
& \quad=\bar{E}\left\{\cos \left(e_{\psi}(k)\right)\right\} \sin (\psi(k)),
\end{aligned}
$$


and that

$$
\begin{aligned}
& \bar{E}\left\{\cos \left(\psi(k)+e_{\psi}(k)\right)\right\}=\bar{E}\left\{\cos (\psi(k)) \cos \left(e_{\psi}(k)\right)-\sin (\psi(k)) \sin \left(e_{\psi}(k)\right)\right\} \\
& =\cos (\psi(k)) \bar{E}\left\{\cos \left(e_{\psi}(k)\right)\right\}-\sin (\psi(k)) \bar{E}\left\{\sin \left(e_{\psi}(k)\right)\right\} \\
& =\bar{E}\left\{\cos \left(e_{\psi}(k)\right)\right\} \cos (\psi(k)),
\end{aligned}
$$

because $e_{\psi}(k)$ following a zero-symmetric probability distribution implies that $\bar{E}\left\{\sin \left(e_{\psi}(k)\right)\right\}=0$. The expected value of $\cos \left(e_{\psi}(k)\right)$ will vary depending on the distribution of $e_{\psi}(k)$ but in general

$$
\bar{E}\left\{\cos \left(e_{\psi}(k)\right)\right\} \triangleq \rho_{e_{\psi}} \neq 1
$$

Consequently,

$$
\begin{aligned}
& \bar{E}\left\{R\left(y_{\psi}(k)\right)\left(\mathbf{y}_{\eta}(k+1)-\mathbf{y}_{\eta}(k-1)\right)\right\} \\
& =\left[\begin{array}{ccc}
\rho_{e_{\psi}} \cos (\psi(k)) & -\rho_{e_{\psi}} \sin (\psi(k)) & 0 \\
\rho_{e_{\psi}} \sin (\psi(k)) & \rho_{e_{\psi}} \cos (\psi(k)) & 0 \\
0 & 0 & 1
\end{array}\right] \cdot\left[\begin{array}{c}
x_{n}(k+1)-x_{n}(k-1) \\
y_{n}(k+1)-y_{n}(k-1) \\
\psi(k+1)-\psi(k-1)
\end{array}\right],
\end{aligned}
$$

which means that the estimates of the surge and sway velocities, $\hat{u}(k)$ and $\hat{v}(k)$, will be biased.

This issue with obtaining an unbiased estimator of the rotation matrix affects alternative ways of forming a velocity state estimator as well, for example ideas based on Kalman filtering or Kalman smoothing. If $\rho_{e_{\psi}}$ happens to be known, a natural modification to the estimator (3.57) is

$$
\hat{v}(k)=\frac{1}{2 T_{s}}\left[\begin{array}{c}
\frac{1}{\rho_{e_{\psi}}} \\
\frac{1}{\rho_{e_{\psi}}} \\
1
\end{array}\right] \circ\left[R\left(y_{\psi}(k)\right)\left(\mathbf{y}_{\eta}(k+1)-\mathbf{y}_{\eta}(k-1)\right)\right] .
$$

Here $\circ$ denotes the Hadamard (dot-wise) product. The estimator (3.64) is unbiased but the requirement of knowing $\rho_{e_{\psi}}$ is not easy to meet. Under a Gaussian assumption, $e_{\psi} \sim \mathcal{N}\left(0, \lambda_{\psi}\right)$, it is the case that $\bar{E}\left\{\cos \left(e_{\psi}(k)\right\}=\exp \left(-\lambda_{\psi} / 2\right)\right.$. Then it is sufficient to know the variance of the measurement noise. An approximation of this variance can be based on physical characteristics of the used sensor. If that information is not available, an estimate can be obtained by for example using the ideas presented in Garcia [2010].

If the variance of the measurement noise is small, the estimator (3.57) will work quite well since the bias decays exponentially. However, for being able to guarantee consistency in a subsequent step of parameter estimation as discussed in 
the remaining chapters of this thesis, it would be necessary to have a consistent estimator of $\rho_{e_{\psi}}$. Such an estimator is not possible to get in the general case and it should therefore be pointed out that the consistency results that are presented in Chapters 4 and 5, only hold as long as the measurements are acquired in the $b$-frame. Notably this does not require the velocities to be measured directly. For example a light detection and ranging solution, usage of acoustic sensors or similar ideas, would provide measurements of the ship's position relative to some object or landmass in the $b$-frame. These measurements could readily be used in a finite difference approximation to form estimates of $\hat{v}$ which are unbiased. 



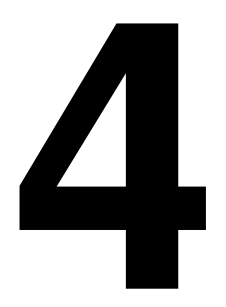

\section{Eliminating Disturbances}

In this chapter, IV estimators for a set of second-order modulus models are suggested which asymptotically eliminate the influence of disturbances on the parameter estimates when the amount of estimation data is large, i.e. IV estimators which are consistent. It is relevant to justify why the potential of the IV method is explored when the two perhaps most commonly used estimation methods in system identification are the Maximum Likelihood (ML) method and PEM. The ML method often gives consistent estimators provided that the corresponding estimation problem can be formulated. However, formulating the ML problem for parameter estimation in general requires prior knowledge about the disturbances' probability distributions. Since the environmental disturbances considered for ships usually are not well-described as white Gaussian processes, the required prior knowledge is not even necessarily restricted to first and second-order moments. This makes the ML method unsuitable in the scenario studied in this work. Another conceivable approach is the LS method. Since the work deals with regression models, the LS estimate coincides with the PEM estimate for a quadratic value function and can readily be formulated. However, such an approach often provides biased estimators under errors-in-variables conditions, a shortcoming of the LS method which will be illustrated in Example 4.1. The IV method can give consistent estimators for many model classes, despite restricted knowledge about the characteristics of the system disturbances. This is the reason why its potential for estimation of parameters in ship models has been analyzed.

Since a continuous-time second-order modulus model like (3.21) can be cast as a discrete-time model with the same type of terms, the results in this chapter are shown by use of a discrete-time state-space system with $n$ states, $m$ inputs and $n$ 
outputs

$$
\begin{aligned}
\mathbf{x}(k+1) & =f\left(\left[\begin{array}{c}
\mathbf{x}(k)+\mathbf{v}(k) \\
\mathbf{u}(k)
\end{array}\right], \theta_{0}\right)+\mathbf{w}(k), \\
\mathbf{y}(k) & =\mathbf{x}(k)+\mathbf{e}(k) .
\end{aligned}
$$

The conventional control nomenclature of Chapter 2 is used and $\mathbf{u}(k)$ is the known control signal, $\mathbf{x}(k)$ is a vector consisting of the latent system states, all of which are measured directly (with noise) and collected in the output vector $\mathbf{y}(k)$. Moreover, $\mathbf{v}(k)$ and $\mathbf{w}(k)$ are external signals that are assumed to be unknown (process disturbances) and $\mathbf{e}(k)$ constitutes an additive measurement error, which is also assumed to be unknown. The system is described by the parameter vector $\theta_{0}$, which does not vary over time. For ease of notation, unit sampling time is assumed without loss of generality.

One major simplification with respect to (3.21), is that the wind and current disturbances are combined into one generic non-additive disturbance, $\mathbf{v}(k)$. It was shown in Chapter 3 that the effect of these environmental disturbances on the ship depends on the attitude of the ship, i.e. on the system state. This state dependence is here neglected. Even in this simplified case, obtaining consistent parameter estimators is challenging.

The analysis in this chapter is divided into two cases. The scenario where the first-order moment of $\mathbf{v}(k)$ is zero will be addressed in Section 4.2 and the scenario where $\mathbf{v}(k)$ is of more general character will be dealt with in Section 4.3. This is followed by two examples, showing the challenges with developing consistent parameter estimators in the two cases. The chapter is concluded by some simulation examples that further illustrate the results. To begin with, a set of assumptions underlying the presented theory are listed.

\subsection{Underlying assumptions}

The following premises regarding the system are assumed to be imposed.

Assumption A1. $f$ is a second-order modulus function in agreement with Definition 3.3 and its structure is known.

Assumption A2. The measurement noise $\mathbf{e}(k)$ is a stationary signal with zero mean and well-defined moments of any order. Also, its amplitude is limited, $-\eta_{e}<\mathbf{e}(k)<\eta_{e}$.

Assumption A3. The process disturbance $\mathbf{w}(k)$ is a stationary signal with welldefined moments of any order. Moreover, $\mathbf{w}(k)$ is independent of $\mathbf{e}(k)$.

Assumption A4. The system is operating in open loop, i.e. the control signal, $\mathbf{u}(k)$, does not depend on the measured states, $\mathbf{y}(k)$, and is consequently assumed to be independent of the disturbances. 
Following Definition 3.3, this system can be expressed as

$$
\begin{aligned}
\mathbf{x}(k+1) & =\Phi^{T}\left(\left[\begin{array}{c}
\mathbf{x}(k)+\mathbf{v}(k) \\
\mathbf{u}(k)
\end{array}\right]\right) \theta_{0}+\mathbf{w}(k), \\
\mathbf{y}(k) & =\mathbf{x}(k)+\mathbf{e}(k) .
\end{aligned}
$$

Since the structure of the true system is known by Assumption A1, it is reasonable to consider the one-step ahead predictor model

$$
\hat{\mathbf{y}}(k \mid \theta)=\Phi^{T}\left(\left[\begin{array}{l}
\mathbf{y}(k-1) \\
\mathbf{u}(k-1)
\end{array}\right]\right) \theta,
$$

for which an additional assumption is made.

Assumption A5. The model structure is globally identifiable according to Definition 2.1.

Assume that $N_{E}$ experiments are performed, where in each $N_{D}$ data points are collected and that for each experiment, $E$, there is an $p \times n$ instrument matrix

$$
\mathbf{Z}_{E}(k)=\left[\zeta_{E, 1}(k) \quad \ldots \quad \zeta_{E, n}(k)\right]
$$

that fulfills the following assumptions.

Assumption A6. $\mathbf{Z}_{E}(k)$ is independent of the noise signals $\mathbf{e}(k), \mathbf{v}(k)$, and $\mathbf{w}(k)$.

Assumption A7. $\bar{E}\left\{\mathbf{Z}_{E}(k)\right\}=0$ and all the moments of higher order are welldefined.

Since an exact solution to (2.18) might not exist, the IV estimate is obtained as the least-squares solution to the system of $p N_{E}$ equations

$$
\left\{\begin{array}{c}
\frac{1}{N_{D}} \sum_{k=1}^{N_{D}} \mathbf{Z}_{1}(k)\left(\mathbf{y}(k)-\Phi^{T}\left(\left[\begin{array}{l}
\mathbf{y}(k-1) \\
\mathbf{u}(k-1)
\end{array}\right]\right) \theta\right)=0, \\
\vdots \\
\frac{1}{N_{D}} \sum_{k=\left(N_{E}-1\right) N_{D}+1}^{N_{E} N_{D}} \mathbf{Z}_{N_{E}}(k)\left(\mathbf{y}(k)-\Phi^{T}\left(\left[\begin{array}{l}
\mathbf{y}(k-1) \\
\mathbf{u}(k-1)
\end{array}\right]\right) \theta\right)=0 .
\end{array}\right.
$$

Finally, it is assumed that when $N_{D} \rightarrow \infty$ the parameters can be determined uniquely, i.e. that the data from all the experiments combined are sufficiently informative.

Assumption A8. $\bar{E}\left\{\left[\begin{array}{c}\mathbf{Z}_{1}(k) \\ \vdots \\ \mathbf{Z}_{N_{E}}(k)\end{array}\right] \Phi^{T}\left(\left[\begin{array}{l}\mathbf{y}(k-1) \\ \mathbf{u}(k-1)\end{array}\right]\right)\right\}$ is full rank. 


\subsection{Zero-mean disturbance}

Now the scenario where the first-order moment of $\mathbf{v}(k)$ is zero will be addressed.

Assumption A9. The process disturbance $\mathbf{v}(k)$ is a white stationary signal with zero mean and well-defined moments of any higher order. Also, its amplitude is limited, $-\eta_{v}<\mathbf{v}(k)<\eta_{v}$ and it is independent of $\mathbf{e}(k)$ as well as of earlier values of $\mathbf{w}(k)$, in other words $\bar{E}\{\mathbf{v}(k) \mathbf{w}(l)\}=\bar{E}\{\mathbf{v}(k)\} \bar{E}\{\mathbf{w}(l)\}, \forall k>l$.

The proposed estimator will be based on an assumption regarding experiment design.

Assumption A10. The input in each experiment is such that it excites the system to the extent that each of its states, $x_{1}(k), \ldots x_{n}(k)$, continuously has an amplitude that is sufficiently well-separated from the origin

$$
\left|x_{i}(k)\right|>\max \left(\eta_{e, i}, \eta_{v, i}\right) \triangleq \eta_{i},
$$

for $k=1, \ldots N_{D}$ and $i=1, \ldots n$.

Performing experiments in accordance with Assumption A10 makes it possible to temporarily treat second-order modulus functions as normal second-order functions, during the analysis of the parameter estimation.

Lemma 4.1. Provided that Assumptions A1-A10 are fulfilled, the IV method defined by (4.5) is a consistent estimator of $\theta$.

Proof: Under the assumptions, the consistency of the IV method can be investigated by analyzing the unbiasedness of the asymptotic IV estimator, i.e. the IV method defined by (4.5) when $N_{D} \rightarrow \infty$. The requirement (2.19a) is already fulfilled by Assumption A8 which means that a sufficient condition for consistency is that also (2.19b) holds, i.e. that

$$
\bar{E}\left\{\mathbf{Z}_{E}(k)\left(\mathbf{y}(k)-\Phi^{T}\left(\left[\begin{array}{c}
\mathbf{y}(k-1) \\
\mathbf{u}(k-1)
\end{array}\right]\right) \theta_{0}\right)\right\}=0,
$$

for all the experiments $E=1, \ldots N_{E}$. By denoting the columns of the regression matrix as $\Phi()=.\left[\begin{array}{lll}\varphi_{1}(.) & \ldots & \varphi_{n}(.)\end{array}\right]$, it can be seen that $(4.6)$ is fulfilled if

$$
\bar{E}\left\{\zeta_{E, i}(k)\left(y_{i}(k)-\varphi_{i}^{T}\left(\left[\begin{array}{l}
\mathbf{y}(k-1) \\
\mathbf{u}(k-1)
\end{array}\right]\right) \theta_{0}\right)\right\}=0,
$$

for $i=1, \ldots n$ and $E=1, \ldots N_{E}$. Here,

$$
\begin{aligned}
y_{i}(k)-\varphi_{i}^{T}\left(\left[\begin{array}{l}
\mathbf{y}(k-1) \\
\mathbf{u}(k-1)
\end{array}\right]\right) \theta_{0}=\varphi_{i}^{T} & \left(\left[\begin{array}{c}
\mathbf{x}(k-1)+\mathbf{v}(k-1) \\
\mathbf{u}(k-1)
\end{array}\right]\right) \theta_{0} \\
& +w_{i}(k-1)+e_{i}(k)-\varphi_{i}^{T}\left(\left[\begin{array}{l}
\mathbf{y}(k-1) \\
\mathbf{u}(k-1)
\end{array}\right]\right) \theta_{0} .
\end{aligned}
$$


Since $\bar{E}\left\{\zeta_{E, i}(k) w_{i}(k-1)\right\}=\bar{E}\left\{\zeta_{E, i}(k) e_{i}(k)\right\}=0$, by A2, A3, A6, and A7 it remains to show that

$$
\bar{E}\left\{\zeta_{E, i}(k)\left(\varphi_{i}^{T}\left(\left[\begin{array}{c}
\mathbf{x}(k-1)+\mathbf{v}(k-1) \\
\mathbf{u}(k-1)
\end{array}\right]\right)-\varphi_{i}^{T}\left(\left[\begin{array}{c}
\mathbf{x}(k-1)+\mathbf{e}(k-1) \\
\mathbf{u}(k-1)
\end{array}\right]\right)\right)\right\}=0,
$$

holds for all $i=1, \ldots, n$. and $E=1, \ldots, N_{E}$. This residual vector will consist of a combination of different kinds of elements. Elements on the form $u_{j},\left|u_{j}\right|, u_{j} u_{l}$, or $u_{j}\left|u_{l}\right|$ are zero since the input is assumed to be perfectly known. Elements on the form $\left|x_{j}\right|$ give

$$
\begin{aligned}
& \bar{E}\left\{\zeta_{E, i}(k)\left[\left|x_{j}(k-1)+v_{j}(k-1)\right|-\left|x_{j}(k-1)+e_{j}(k-1)\right|\right]\right\} \\
& \quad=\bar{E}\left\{\zeta_{E, i}(k)\left[x_{j}(k-1)+v_{j}(k-1)-\left(x_{j}(k-1)+e_{j}(k-1)\right)\right]\right\}=0,
\end{aligned}
$$

if $x_{j}>\eta_{j}$. This follows by A2, A6, A9, and A10. For the case when $x_{j}<-\eta_{j}$ only the sign of the expression changes. Cross-elements on the form $x_{j}\left|u_{l}\right|$ give

$$
\begin{aligned}
& \bar{E}\left\{\zeta_{E, i}(k)\left(\left(x_{j}(k-1)+v_{j}(k-1)\right)\left|u_{l}(k-1)\right|-\left(x_{j}(k-1)+e_{j}(k-1)\right)\left|u_{l}(k-1)\right|\right)\right\} \\
& \quad=\bar{E}\left\{\zeta_{E, i}(k)\left|u_{l}(k-1)\right|\left(v_{j}(k-1)-e_{j}(k-1)\right)\right\}=0,
\end{aligned}
$$

which follows from A2, A4, A6, and A9. Cross-elements on the form $u_{j}\left|x_{l}\right|$ give

$$
\begin{aligned}
\bar{E} & \left\{\zeta_{E, i}(k)\left(u_{j}(k-1)\left|x_{l}(k-1)+v_{l}(k-1)\right|-u_{j}(k-1)\left|x_{l}(k-1)+e_{l}(k-1)\right|\right)\right\} \\
& =\bar{E}\left\{\zeta_{E, i}(k) u_{j}(k-1)\left(x_{l}(k-1)+v_{l}(k-1)-\left(x_{l}(k-1)+e_{l}(k-1)\right)\right)\right\} \\
& =\bar{E}\left\{\zeta_{E, i}(k) u_{j}(k-1)\left(v_{l}(k-1)-e_{l}(k-1)\right)\right\}=0
\end{aligned}
$$

if $x_{l}>\eta_{l}$. This follows by A2, A4, A6, A9, and A10. For the case when $x_{l}<-\eta_{l}$, only the sign of the expression changes. Finally elements on the form $x_{j}\left|x_{l}\right|$ give

$$
\begin{aligned}
& \bar{E}\left\{\zeta _ { E , i } ( k ) \left(\left(x_{j}(k-1)+v_{j}(k-1)\right)\left|x_{l}(k-1)+v_{l}(k-1)\right|-\left(x_{j}(k-1)+e_{j}(k-1)\right)\right.\right. \\
& \left.\left.\cdot\left|x_{l}(k-1)+e_{l}(k-1)\right|\right)\right\}=\bar{E}\left\{\zeta _ { E , i } ( k ) \left(x_{j}(k-1) x_{l}(k-1)+x_{j}(k-1) v_{l}(k-1)\right.\right. \\
& +v_{j}(k-1) x_{l}(k-1)+v_{j}(k-1) v_{l}(k-1)-x_{j}(k-1) x_{l}(k-1)-x_{j}(k-1) e_{l}(k-1) \\
& \left.\left.-e_{j}(k-1) x_{l}(k-1)-e_{j}(k-1) e_{l}(k-1)\right)\right\}=\bar{E}\left\{\zeta _ { E , i } ( k ) \left(x _ { j } ( k - 1 ) \left(v_{l}(k-1)\right.\right.\right. \\
& \left.-e_{l}(k-1)\right)+x_{l}(k-1)\left(v_{j}(k-1)-e_{j}(k-1)\right)+v_{j}(k-1) v_{l}(k-1) \\
& \left.\left.-e_{j}(k-1) e_{l}(k-1)\right)\right\}=0,
\end{aligned}
$$


if $x_{l}>\eta_{l}$. This follows by A2, A3, A4, A6, A7, A9, and A10. For the case when $x_{l}<-\eta_{l}$, only the sign of the expression changes.

First and second-order elements without the modulus operator can be seen to equal zero following to the same type of reasoning. Hence, all elements in (4.8) will be zero, regardless of $i, j, l$, and $E$. Conclusively (4.6) is fulfilled so the estimator for $\theta$ is consistent. This concludes the proof.

Remark 4.1. It is important that the input is informative such that the parameters can be determined uniquely. For example, if both the regressors $x_{j}\left|x_{l}\right|$ and $x_{l}\left|x_{j}\right|$ are present in $\varphi_{i}$, both experiments where $x_{j}$ and $x_{l}$ are of the same sign and experiments where they are of opposite sign are needed.

Remark 4.2. If a whole experiment does not fulfill Assumption A10, it is possible to form new shorter datasets, only including the parts that do. Only two sequential data points are needed from each set in order to contribute to the estimate. This is due to the nature of the system following Definition 3.3, where the state at sample time $k$ only depends on the state and the input at sample time $k-1$.

Remark 4.3. Only the biggest of $\eta_{i, e}$ and $\eta_{i, v}$ is necessary to consider when the experiment is designed.

\subsection{General disturbance}

Now the scenario where the process disturbance $\mathbf{v}(k)$ is of more general character will be addressed. It will however be assumed that an independent measurement of $\mathbf{v}(k)$ is available. Therefore, let $\mathbf{y}_{1}(k)=\mathbf{x}(k)+\mathbf{e}_{1}(k),-\eta_{e_{1}}<\mathbf{e}_{1}(k)<\eta_{e_{1}}$, denote the original state measurement.

Assumption A11. The process disturbance $\mathbf{v}(k)$ is a signal with well-defined moments of any order and its amplitude is limited, $-\eta_{v}<\mathbf{v}(k)<\eta_{v}$.

Assumption A12. An unbiased measurement $\mathbf{y}_{2}(k)=\mathbf{v}(k)+\mathbf{e}_{2}(k)$ is available. The measurement noise $\mathbf{e}_{2}(k)$ is a stationary signal with zero mean and welldefined moments of any order. Also, its amplitude is limited, $-\eta_{e_{2}}<\mathbf{e}_{2}(k)<\eta_{e_{2}}$.

Assumption A13. $\mathbf{Z}_{E}(k)$ is independent of $\mathbf{e}_{2}(k)$.

Assumption A14. The measurement disturbances, $\mathbf{e}_{1}(k)$ and $\mathbf{e}_{2}(k)$, are independent of the process disturbances, $\mathbf{v}(k)$ and $\mathbf{w}(k)$.

Assumption A15. The input in each experiment is such that it excites the system to the extent that each of its states, $x_{1}(k), \ldots x_{n}(k)$, continuously has an amplitude that is sufficiently well-separated from the origin

$$
\left|x_{i}(k)\right|>\eta_{e_{1}, i}+\eta_{e_{2}, i}+\eta_{v, i} \triangleq \eta_{i, \check{e}}+\eta_{v, i}
$$

for $k=1, \ldots N_{D}$ and $i=1, \ldots n$. 
Let $\check{\mathbf{y}}(k)=\mathbf{y}_{1}(k)+\mathbf{y}_{2}(k)$ and consider the predictor

$$
\hat{\mathbf{y}}(k \mid \theta)=\Phi^{T}\left(\left[\begin{array}{c}
\check{\mathbf{y}}(k-1) \\
\mathbf{u}(k-1)
\end{array}\right]\right) \theta .
$$

Define the IV estimator as the least-squares solution to the system of $p N_{E}$ equations

$$
\left\{\begin{array}{c}
\frac{1}{N_{D}} \sum_{k=1}^{N_{D}} \mathbf{Z}_{1}(k)\left(\mathbf{y}_{1}(k)-\Phi^{T}\left(\left[\begin{array}{l}
\check{\mathbf{y}}(k-1) \\
\mathbf{u}(k-1)
\end{array}\right]\right) \theta\right)=0, \\
\vdots \\
\frac{1}{N_{D}} \sum_{k=\left(N_{E}-1\right) N_{D}+1}^{N_{E} N_{D}} \mathbf{Z}_{N_{E}}(k)\left(\mathbf{y}_{1}(k)-\Phi^{T}\left(\left[\begin{array}{l}
\check{\mathbf{y}}(k-1) \\
\mathbf{u}(k-1)
\end{array}\right]\right) \theta\right)=0 .
\end{array}\right.
$$

Lemma 4.2. Provided that Assumptions A1-A8 and A11-A15 are fulfilled, the IV method defined by (4.14) is a consistent estimator of $\theta$.

Proof: Under the assumptions, the consistency of the IV method can be investigated by analyzing the unbiasedness of the asymptotic IV estimator, i.e. the IV method defined by (4.14) when $N_{D} \rightarrow \infty$. The requirement (2.19a) is already fulfilled by $\mathrm{A} 8$ which means that a sufficient condition for consistency is that also (2.19b) holds, i.e. that

$$
\bar{E}\left\{\mathbf{Z}_{E}(k)\left(\mathbf{y}_{1}(k)-\Phi^{T}\left(\left[\begin{array}{l}
\check{\mathbf{y}}(k-1) \\
\mathbf{u}(k-1)
\end{array}\right]\right) \theta_{0}\right)\right\}=0,
$$

for all the experiments $E=1, \ldots N_{E}$. Again, by denoting the columns of the regression matrix as $\Phi()=.\left[\begin{array}{lll}\varphi_{1}(.) & \ldots & \varphi_{n}(.)\end{array}\right]$, it can be seen that (4.15) is fulfilled if

$$
\bar{E}\left\{\zeta_{E, i}(k)\left(y_{1, i}(k)-\varphi_{i}^{T}\left(\left[\begin{array}{c}
\check{\mathbf{y}}(k-1) \\
\mathbf{u}(k-1)
\end{array}\right]\right) \theta_{0}\right)\right\}=0,
$$

for $i=1, \ldots n$ and $E=1, \ldots N_{E}$. Here

$$
\begin{aligned}
y_{1, i}(k)-\varphi_{i}^{T}\left(\left[\begin{array}{c}
\check{\mathbf{y}}(k-1) \\
\mathbf{u}(k-1)
\end{array}\right]\right) \theta_{0} & =\varphi_{i}^{T}\left(\left[\begin{array}{c}
\mathbf{x}(k-1)+\mathbf{v}(k-1) \\
\mathbf{u}(k-1)
\end{array}\right]\right) \theta_{0} \\
& +w_{i}(k-1)+e_{1, i}(k)-\varphi_{i}^{T}\left(\left[\begin{array}{c}
\check{\mathbf{y}}(k-1) \\
\mathbf{u}(k-1)
\end{array}\right]\right) \theta_{0} .
\end{aligned}
$$

Since $\bar{E}\left\{\zeta_{E, i}(k) w_{i}(k-1)\right\}=\bar{E}\left\{\zeta_{E, i}(k) e_{1, i}(k)\right\}=0$, by A2, A3, A6, and A7 it remains to show that

$$
\bar{E}\left\{\zeta_{E, i}(k)\left(\varphi_{i}^{T}\left(\left[\begin{array}{c}
\mathbf{x}(k-1)+\mathbf{v}(k-1) \\
\mathbf{u}(k-1)
\end{array}\right]\right)-\varphi_{i}^{T}\left(\left[\begin{array}{l}
\check{\mathbf{y}}(k-1) \\
\mathbf{u}(k-1)
\end{array}\right]\right)\right)\right\}=0,
$$


holds for all $i=1, \ldots, n$. and $E=1, \ldots, N_{E}$. Let

$$
\begin{aligned}
& \mathbf{r}(k) \triangleq \mathbf{x}(k)+\mathbf{v}(k), \\
& \check{\mathbf{e}}(k) \triangleq \mathbf{e}_{1}(k)+\mathbf{e}_{2}(k) .
\end{aligned}
$$

Then it holds that $\check{\mathbf{y}}(k)=\mathbf{r}(k)+\check{\mathbf{e}}(k)$. Further note that A15 and (4.19) implies that

$$
\left|x_{i}(k)\right|>\eta_{\check{e}, i}+\left|v_{i}(k)\right| \Longrightarrow\left|r_{i}(k)\right|>\eta_{\check{e}, i} .
$$

Verifying (4.18) is equivalent to verifying

$$
\bar{E}\left\{\zeta_{E, i}(k)\left(\varphi_{i}^{T}\left(\left[\begin{array}{l}
\mathbf{r}(k-1) \\
\mathbf{u}(k-1)
\end{array}\right]\right)-\varphi_{i}^{T}\left(\left[\begin{array}{c}
\mathbf{r}(k-1)+\check{\mathbf{e}}(k-1) \\
\mathbf{u}(k-1)
\end{array}\right]\right)\right)\right\}=0 .
$$

The remainder of the proof is similar to that of Lemma 4.1 but is still included for completeness. The residual vector above will consist of a combination of different kinds of elements. Elements on the form $u_{j},\left|u_{j}\right|, u_{j} u_{l}$ or $u_{j}\left|u_{l}\right|$ are zero since the input is assumed to be perfectly known. Elements on the form $\left|r_{j}\right|$ give

$$
\begin{aligned}
& \bar{E}\left\{\zeta_{E, i}(k)\left(\left|r_{j}(k-1)\right|-\left|r_{j}(k-1)+\check{e}_{j}(k-1)\right|\right)\right\} \\
& =\bar{E}\left\{\zeta_{E, i}(k)\left(r_{j}(k-1)-\left(r_{j}(k-1)+\check{e}_{j}(k-1)\right)\right)\right\}=0,
\end{aligned}
$$

if $r_{j}>\eta_{\check{e}, j}$. This follows by A2, A6, A12, A13 and A15. For the case when $r_{j}<$ $-\eta_{\tilde{e}, j}$ only the sign of the expression changes. Cross-elements on the form $r_{j}\left|u_{l}\right|$ give

$$
\begin{aligned}
\bar{E} & \left\{\zeta_{E, i}(k)\left(r_{j}(k-1)\left|u_{l}(k-1)\right|-\left(r_{j}(k-1)+\check{e}_{j}(k-1)\left|u_{l}(k-1)\right|\right)\right\}\right. \\
& =-\bar{E}\left\{\zeta_{E, i}(k)\left|u_{l}(k-1)\right| \check{e}_{j}(k-1)\right\}=0,
\end{aligned}
$$

which follows from A2, A4, A6, A12 and A13. Cross-elements on the form $u_{j}\left|r_{l}\right|$ give

$$
\begin{aligned}
\bar{E} & \left\{\zeta_{E, i}(k)\left(u_{j}(k-1)\left|r_{l}(k-1)\right|-u_{j}(k-1)\left|r_{l}(k-1)+\check{e}_{l}(k-1)\right|\right)\right\} \\
& =\bar{E}\left\{\zeta_{E, i}(k) u_{j}(k-1)\left(r_{l}(k-1)-\left(r_{l}(k-1)+\check{e}_{l}(k-1)\right)\right)\right\} \\
& =-\bar{E}\left\{\zeta_{E, i}(k) u_{j}(k-1) \check{e}_{l}(k-1)\right\}=0,
\end{aligned}
$$

if $r_{l}>\eta_{\check{e}, l}$. This follows by A2, A4, A6, A12, A13, A13 and A15. For the case when $x_{l}<-\eta_{\check{e}, l}$, only the sign of the expression changes. Finally, elements on the 
form $r_{j}\left|r_{l}\right|$ give

$$
\begin{aligned}
\bar{E} & \left\{\zeta_{E, i}(k)\left(r_{j}(k-1)\left|r_{l}(k-1)\right|-\left(r_{j}(k-1)+\check{e}_{j}(k-1)\right)\left|r_{l}(k-1)+\check{e}_{l}(k-1)\right|\right)\right\} \\
& =\bar{E}\left\{\zeta_{E, i}(k)\left(r_{j}(k-1) r_{l}(k-1)-\left(r_{j}(k-1)+\check{e}_{j}(k-1)\right)\left(r_{l}(k-1)+\check{e}_{l}(k-1)\right)\right)\right\} \\
& =-\bar{E}\left\{\zeta_{E, i}(k)\left(r_{j}(k-1) \check{e}_{l}(k-1)+\check{e}_{j}(k-1) r_{l}(k-1)+\check{e}_{j}(k-1) \check{e}_{l}(k-1)\right)\right\}=0,
\end{aligned}
$$

if $r_{l}>\eta_{\check{e}, l}$. This follows by A2, A4, A6, A7, A11, A12, A13, A14 and A15. For the case when $x_{l}<-\eta_{\check{e}, l}$, only the sign of the expression changes.

First and second-order elements without the modulus operator can be seen to equal zero following to the same type of reasoning. Hence, all elements in (4.18) will be zero, regardless of $i, j, l$, and $E$. Conclusively, (4.15) is fulfilled so the estimator for $\theta$ is consistent. This concludes the proof.

Remark 4.4. In this scenario, it was never assumed that the disturbances, $\mathbf{e}_{1}(k), \mathbf{e}_{2}(k)$, $\mathbf{v}(k)$, and $\mathbf{w}(k)$, are white. In fact, $\mathbf{v}(k)$ can even include a deterministic time-dependent component.

Remark 4.5. Assumption A15 is a more restricting condition for the experiment design than Assumption A10.

Remark 4.6. The main idea of the proof is to consider the aggregation $\mathbf{r}(k)=\mathbf{x}(k)+\mathbf{v}(k)$ as state, temporarily during estimation. Instead of adding a second measurement of the disturbance $\mathbf{v}(k)$ it is possible to add a measurement of the aggregated state $\mathbf{r}(k)$. In general, two out of the three quantities $\mathbf{x}(k), \mathbf{v}(k)$ and $\mathbf{r}(k)$ need to be measured.

\subsection{Motivating examples}

Obtaining a consistent parameter estimator of even a small scale single-input single-output (SISO) second-order modulus system can prove to be a bit cumbersome. This will be illustrated by two examples. In the first example, the non-additive disturbance $\mathbf{v}(k)$ is assumed to be identically zero. Conducting experiments where the input signal has a static offset of sufficient amplitude and forcing instruments to have zero mean can already in this case improve the accuracy of an IV estimator. In the second example a non-additive disturbance is included. In that case, it is only possible to achieve consistency if the disturbance has zero mean or is measured.

Example 4.1

Consider the SISO system

$$
\begin{aligned}
x(k+1) & =n_{0} x(k)|x(k)|+f_{0} u(k)+w(k), \\
y(k) & =x(k)+e(k),
\end{aligned}
$$


where the two noise sources are mutually independent, white, stationary, uniformly distributed stochastic processes with zero mean and $-\eta_{w}<w(k)<\eta_{w}$, $-\eta_{e}<e(k)<\eta_{e}$. Assume that the input is Gaussian and white, also with zero mean and that the system is operating in open loop, i.e. that the input, $u(k)$, is not dependent of the measured state, $y(k)$, and consequently assumed to be independent of the noise signals, $w(k)$ and $e(k)$. Let $\theta_{0}=\left[\begin{array}{ll}n_{0} & f_{0}\end{array}\right]^{T}$ denote the true system parameter vector. A simple way of modelling this system is to ignore the fact that only noisy measurements of $x(k)$ are available and to consider the one-step ahead predictor model

$$
\hat{y}(k \mid \theta)=[y(k-1)|y(k-1)| u(k-1)]\left[\begin{array}{l}
n \\
f
\end{array}\right] \triangleq \varphi^{T}(k) \theta .
$$

In agreement with (2.17), the asymptotic LS estimator of the system parameters can be obtained as

$$
\lim _{N \rightarrow \infty}^{\text {w.p.1 }} \hat{\theta}_{N}^{L S}=\bar{E}\left\{\varphi(k) \varphi^{T}(k)\right\}^{-1} \bar{E}\{\varphi(k) y(k)\} .
$$

Due to the causality of the system and the fact that the input and noise sequences are white and have zero mean, it is assumed to be the case that

$$
\begin{gathered}
\bar{E}\{u(k) x(l)\}=0 \forall k \geq l, \\
\bar{E}\{e(k) x(l)\}=0, \forall k, l, \\
\bar{E}\{w(k) x(l)\}=0, \forall k \geq l .
\end{gathered}
$$

Using this and the fact that the system is operating in open loop gives

$$
\begin{aligned}
\bar{E}\left\{\varphi(k) \varphi^{T}(k)\right\} & =\left[\begin{array}{cc}
\bar{E}\left\{y(k-1)^{4}\right\} & 0 \\
0 & \bar{E}\left\{u(k-1)^{2}\right\}
\end{array}\right], \\
\bar{E}\{\varphi(k) y(k)\} & =\left[\begin{array}{c}
\bar{E}\{y(k) y(k-1)|y(k-1)|\} \\
\bar{E}\{u(k-1) y(k)\}
\end{array}\right] .
\end{aligned}
$$

It is the case that

$$
\bar{E}\left\{y(k-1)^{4}\right\}=\bar{E}\left\{x(k-1)^{4}+6 x(k-1)^{2} e(k-1)^{2}+e(k-1)^{4}\right\},
$$

because the expected value of any odd moment of a zero-symmetric distribution (if it exists) is zero. This holds for $x(k)$ and $e(k)$ alike. Also,

$$
\begin{aligned}
& \bar{E}\{y(k) y(k-1)|y(k-1)|\}=\bar{E}\left\{\left(n_{0} x(k-1)|x(k-1)|+f_{0} u(k-1)+w(k-1)+e(k)\right)\right. \\
& \cdot(x(k-1)+e(k-1))|x(k-1)+e(k-1)|\} \\
& =n_{0} \bar{E}\{x(k-1)|x(k-1)|(x(k-1)+e(k-1))|x(k-1)+e(k-1)|\},
\end{aligned}
$$


where the properties that $u(k), w(k)$ and $e(k)$ are white, mutually independent and have zero mean were used. Lastly,

$$
\begin{aligned}
\bar{E}\{u(k-1) y(k)\} & =\bar{E}\left\{u(k-1)\left(n_{0} x(k-1)|x(k-1)|+f_{0} u(k-1)+w(k-1)+e(k)\right)\right\} \\
& =f_{0} \bar{E}\left\{u(k-1)^{2}\right\},
\end{aligned}
$$

making use of the same properties. All in all this means that

$$
\lim _{N \rightarrow \infty} \hat{\theta}_{N}^{L S}=\left[\begin{array}{c}
\frac{n_{0} \bar{E}\{x|x|(x+e)|x+e|\}}{\bar{E}\left\{x^{4}+6 x^{2} e^{2}+e^{4}\right\}} \\
f_{0}
\end{array}\right],
$$

where the time index was dropped for simplified notation. This means that in presence of measurement noise, $e \neq 0$, the LS estimator is inconsistent. This result is expected since it is a known fact that the LS estimate is inconsistent under errors-in-variables (EIV) conditions, see for example Söderström [2007].

The IV estimator is given by (2.18). It has been shown in Gilson et al. [2011], that having the instrument vector represent a noise-free version of the regression vector is a good choice, at least in the linear case. Assume that completely noisefree regressors are available for use as instruments

$$
\zeta(k)=[x(k-1)|x(k-1)| \quad u(k-1)]^{T} .
$$

If $\bar{E}\left\{\zeta(k) \varphi^{T}(k)\right\}$ is nonsingular, the asymptotic IV estimator can be obtained as

$$
\lim _{N \rightarrow \infty} \hat{\theta}_{N}^{I V}=\bar{E}\left\{\zeta(k) \varphi^{T}(k)\right\}^{-1} \bar{E}\{\zeta(k) y(k)\}=\left[\begin{array}{c}
\bar{E}\{y(k) x(k-1)|x(k-1)|\} \\
\bar{E}\{x(k-1)|x(k-1)| y(k-1)|y(k-1)|\} \\
\frac{\bar{E}\{u(k-1) y(k)\}}{\bar{E}\left\{u(k-1)^{2}\right\}}
\end{array} .\right.
$$

For the new expressions, it holds that

$$
\begin{aligned}
& \bar{E}\{y(k) x(k-1)|x(k-1)|\}=\bar{E}\left\{\left(n_{0} x(k-1)|x(k-1)|+f_{0} u(k-1)\right.\right. \\
& +w(k-1)+e(k)) x(k-1)|x(k-1)|\}=n_{0} \bar{E}\left\{x(k-1)^{4}\right\}
\end{aligned}
$$

and

$$
\begin{aligned}
& \bar{E}\{x(k-1)|x(k-1)| y(k-1)|y(k-1)|\} \\
& =\bar{E}\{x(k-1)|x(k-1)|(x(k-1)+e(k-1))|x(k-1)+e(k-1)|\},
\end{aligned}
$$

so that, again omitting the time index

$$
\lim _{N \rightarrow \infty}^{\text {w.p.1 }} \hat{\theta}_{N}^{I V}=\left[\begin{array}{c}
\frac{n_{0} \bar{E}\left\{x^{4}\right\}}{\bar{E}\{x|x|(x+e)|x+e|\}} \\
f_{0}
\end{array}\right],
$$


which is inconsistent as well. For an IV estimator to be consistent, (2.19b) must hold. For this system this means that

$$
\begin{aligned}
& \bar{E}\left\{\zeta ( k ) \left(n_{0} x(k-1)|x(k-1)|+w(k-1)+e(k)\right.\right. \\
& \left.\left.\quad-n_{0}(x(k-1)+e(k-1))|x(k-1)+e(k-1)|\right)\right\}=0 .
\end{aligned}
$$

If the instruments are uncorrelated with $w(k)$ and $e(k)$ this implies that

$$
\bar{E}\{\zeta(k)(x(k-1)|x(k-1)|-(x(k-1)+e(k-1))|x(k-1)+e(k-1)|)\}=0,
$$

which is not easily fulfilled, while keeping $\bar{E}\left\{\zeta(k) \varphi^{T}(k)\right\}$ nonsingular, for any choice of $\zeta(k)$. This means that despite the fact that the instruments are uncorrelated with the noise signals, the IV estimator is inconsistent.

However, an inconsistent estimate can be avoided by using an input with an offset. Remember that the amplitude of the measurement noise has an upper bound, $|e(k)|<\eta_{e}$. The input should be such that it excites the system to the extent that its state, $x(k)$, has an amplitude that is bigger than the worst-case amplitude of the measurement noise. Consider the case where the input $u(k)=\bar{u}+\tilde{u}(k)$ is applied, where $\tilde{u}(k)$ is uniformly distributed with zero mean, $-\eta_{\tilde{u}}<\tilde{u}(k)<\eta_{\tilde{u}}$. If the system is stable this will yield an output that, with the notation $\bar{E}\{x(k)\}=\bar{x}$, can be written like

$$
x(k)=\bar{x}+\tilde{x}(k), \quad \bar{E}\{\tilde{x}(k)\}=0 .
$$

Further, assume that $\bar{u}>0, \bar{x}>0$ and

$$
x(k)=\bar{x}+\tilde{x}(k)>\eta_{e}>0 .
$$

Under the stated circumstances it can be concluded that $x(k)+e(k)>0$ and consequently that

$$
\begin{aligned}
\lim _{N \rightarrow \infty}^{\text {w.p.1 }} & \frac{1}{N} \sum_{k=1}^{N} \zeta(k)\left(y(k)-\varphi^{T}(k) \theta_{0}\right) \\
& =\bar{E}\left\{\zeta(k)\left(w(k-1)+e(k)-2 n_{0} x(k-1) e(k-1)-e(k-1)^{2}\right)\right\} .
\end{aligned}
$$

This will equal zero for all instruments that are independent of the noise signals, $w(k)$ and $e(k)$, while also fulfilling $\bar{E}\{\zeta(k)\}=0$. For example, the instrument vector

$$
\begin{aligned}
\zeta(k) & =\left[\begin{array}{lll}
x(k-1)^{2}-\bar{E}\left\{x(k-1)^{2}\right\} & u(k-1)-\bar{E}\{u(k-1)\}
\end{array}\right]^{T} \\
& =\left[\begin{array}{ll}
\tilde{x}(k-1)^{2}+2 \bar{x} \tilde{x}(k-1)-\bar{E}\left\{\tilde{x}(k-1)^{2}\right\} & \tilde{u}(k-1)
\end{array}\right]^{T},
\end{aligned}
$$


gives

$$
\begin{aligned}
\bar{E}\left\{\zeta(k) \varphi^{T}(k)\right\} & =\left[\begin{array}{cc}
m_{\tilde{x}} & 0 \\
0 & \bar{E}\left\{\tilde{u}^{2}\right\}
\end{array}\right], \\
\bar{E}\{\zeta(k) y(k)\} & =\left[\begin{array}{c}
n_{0} m_{\tilde{x}} \\
f_{0} \bar{E}\left\{\tilde{u}^{2}\right\}
\end{array}\right],
\end{aligned}
$$

where $m_{\tilde{x}}=\bar{E}\left\{\tilde{x}^{4}\right\}+4 \bar{x} \bar{E}\left\{\tilde{x}^{3}\right\}+4 \bar{x}^{2} \bar{E}\left\{\tilde{x}^{2}\right\}-\bar{E}\left\{\tilde{x}^{2}\right\}^{2}$. These computations are also based on the fact that $\tilde{x}(k)$ and $\tilde{u}(k)$ are independent. As long as the input is persistently exciting so that $\bar{E}\left\{\zeta(k) \varphi^{T}(k)\right\}$ is invertible, the asymptotic parameter estimator is consistent

$$
\lim _{N \rightarrow \infty}^{\text {w.p. } 1} \hat{\theta}_{N}^{I V}=\bar{E}\left\{\zeta(k) \varphi^{T}(k)\right\}^{-1} \bar{E}\{\zeta(k) y(k)\}=\left[\begin{array}{l}
n_{0} \\
f_{0}
\end{array}\right]=\theta_{0} .
$$

Example 4.2

Assume that data is generated based on the SISO system

$$
\begin{aligned}
x(k+1) & =n_{0}(x(k)+v(k))|x(k)+v(k)|+f_{0} u(k)+w(k), \\
y_{1}(k) & =x(k)+e_{1}(k),
\end{aligned}
$$

where $v(k)=\bar{v}+\tilde{v}(k)$ and $\tilde{v}(k)$ is white, uniformly distributed $-\eta_{\tilde{v}}<\tilde{v}(k)<\eta_{\tilde{v}}$ and has zero mean, $w(k)$ is a stationary signal with well-defined moments of any order and $e_{1}(k)$ is uniformly distributed with zero mean, $-\eta_{e_{1}}<e_{1}(k)<\eta_{e_{1}}$. Consider the predictor

$$
\hat{y}(k \mid \theta)=\left[y_{1}(k-1)\left|y_{1}(k-1)\right| \quad u(k-1)\right]\left[\begin{array}{l}
n \\
f
\end{array}\right] \triangleq \varphi^{T}(k) \theta,
$$

and the input $u(k)=\bar{u}+\tilde{u}(k)$, where $\tilde{u}(k)$ is uniformly distributed with zero mean, $-\eta_{\tilde{u}}<\tilde{u}(k)<\eta_{\tilde{u}}$. If the system is stable this will yield an output that, with the notation $\bar{E}\{x(k)\}=\bar{x}$, can be written as

$$
x(k)=\bar{x}+\tilde{x}(k), \bar{E}\{\tilde{x}(k)\}=0 .
$$

For simplicity assume that $\bar{u}>0, \bar{x}>0$ and

$$
x(k)=\bar{x}+\tilde{x}(k)>\max \left(|\bar{v}|+\eta_{\tilde{v}}, \eta_{e_{1}}\right)>0 .
$$

Under the stated circumstances it can be concluded that $x(k)+v(k)>0$ and $x(k)+$ $e_{1}(k)>0$ and as a consequence, all occurrences of the modulus operator, both in the system and in the predictor can be ignored. Provided that the input is informative such that (2.19a) holds, the consistency of an IV estimator can be 
studied by the fulfillment of (2.19b), which left-hand side in this situation is

$$
\begin{aligned}
& \bar{E}\left\{\zeta(k)\left(y_{1}(k)-\varphi^{T}(k) \theta_{0}\right)\right\}=\bar{E}\left\{\zeta ( k ) \left(n_{0}(x(k-1)+v(k-1))^{2}+f_{0} u(k-1)\right.\right. \\
& \left.\left.+w(k-1)+e_{1}(k)-n_{0}\left(x(k-1)+e_{1}(k-1)\right)^{2}-f_{0} u(k-1)\right)\right\} \\
& \quad=2 n_{0} \bar{E}\left\{\zeta(k) x(k-1)\left(v(k-1)-e_{1}(k-1)\right)\right\}+n_{0} \bar{E}\left\{\zeta(k)\left(v(k-1)^{2}-e_{1}(k-1)^{2}\right)\right\} \\
& +\bar{E}\{\zeta(k) w(k-1)\}+\bar{E}\left\{\zeta(k) e_{1}(k)\right\}=2 n_{0} \bar{v} \bar{E}\{\zeta(k) x(k-1)\},
\end{aligned}
$$

where the last equality follows if $u(k)$ and $\zeta(k)$ are independent of the disturbances, $\bar{E}\{\zeta(k)\}=0$, and the disturbances are mutually independent. If (2.19a) holds, it is the case that $\bar{E}\{\zeta(k) x(k-1)\} \neq 0$. This means that the estimator will be consistent if $\bar{v}=0$ but not otherwise.

As shown in Section 4.3, one way to get consistency in the general case when $\bar{v} \neq 0$, is to add a second sensor for measuring the disturbance, $y_{2}(k)=v(k)+e_{2}(k)$. Here it will be assumed that also $e_{2}(k)$ is uniformly distributed with zero mean, $-\eta_{e_{2}}<e_{2}(k)<\eta_{e_{2}}$. Now consider the aggregated-state predictor

$$
\hat{y}_{r}(k \mid \theta)=[\check{y}(k-1)|\check{y}(k-1)| u(k-1)]\left[\begin{array}{l}
n \\
f
\end{array}\right] \triangleq \varphi_{r}^{T}(k) \theta,
$$

where $\check{y}(k)=x(k)+v(k)+\check{e}(k)$ and $\check{e}(k)=e_{1}(k)+e_{2}(k)$. In order to be able to ignore the modulus functions it is assumed that

$$
x(k)>|\bar{v}|+\eta_{\tilde{v}}+\eta_{e_{1}}+\eta_{e_{2}}
$$

This gives another left-hand side of (2.19b)

$$
\begin{aligned}
& \bar{E}\left\{\zeta(k)\left(y_{1}(k)-\varphi_{r}^{T}(k) \theta_{0}\right)\right\}=\bar{E}\left\{\zeta ( k ) \left(n_{0}(x(k-1)+v(k-1))^{2}+f_{0} u(k-1)\right.\right. \\
& \left.\left.+w(k-1)+e_{1}(k)-n_{0}(x(k-1)+v(k-1)+\check{e}(k-1))^{2}-f_{0} u(k-1)\right)\right\} \\
& =-2 n_{0} \bar{E}\{\zeta(k)(x(k-1)+v(k-1)) \check{e}(k-1)\}-n_{0} \bar{E}\left\{\zeta(k) \check{e}(k-1)^{2}\right\}+\bar{E}\{\zeta(k) w(k-1)\} \\
& +\bar{E}\left\{\zeta(k) e_{1}(k)\right\}=-2 n_{0} \bar{E}\{\check{e}(k-1)\} \bar{E}\{\zeta(k)(v(k-1)+x(k-1))\}=0,
\end{aligned}
$$

where the last equality holds provided that the same assumptions regarding instrument vector, input, and disturbances are imposed. This means that the estimator is consistent. 


\subsection{Simulations}

In order to further illustrate the results, some simulations were performed using the SISO system

$$
\begin{aligned}
x(k+1) & =n_{0}(x(k)+v(k))|x(k)+v(k)|+f_{0} u(k)+w(k), \\
y_{1}(k) & =x(k)+e_{1}(k), \\
y_{2}(k) & =v(k)+e_{2}(k) .
\end{aligned}
$$

This system was also used in the arithmetic examples above. It is sufficiently simple and transparent for analysis but yet the estimation of its parameters is a non-trivial task, which includes all the challenges discussed in this chapter. In the simulation study, the system parameters were $n_{0}=-0.1$ and $f_{0}=1$.

The four noise sources were sampled from Gaussian distributions

$$
\begin{aligned}
w(k) & \sim \mathcal{N}(0.1,0.1), \\
v(k) & \sim \mathcal{N}(\bar{v}, 0.25), \\
e_{1}(k) & \sim \mathcal{N}(0,0.1), \\
e_{2}(k) & \sim \mathcal{N}(0,0.1),
\end{aligned}
$$

which means that neither the distributions of the measurement noises nor of the non-additive process disturbance did have finite support. This choice was made in order to test the robustness of the discussed methods. Four sets of Monte Carlo simulations were carried out. In each Monte Carlo iteration, $N=5000$ data points were used for parameter estimation. This procedure was repeated 500 times per set, using new noise sequences.

Three estimators were compared in the simulations, one LS estimator, and two IV estimators. The IV estimators differed by having zero-mean instruments or not. The estimators will be denoted as $\hat{\theta}_{N}^{L S}, \hat{\theta}_{N}^{I V_{1}}$ and $\hat{\theta}_{N}^{I V_{2}}$, where the last one uses zero-mean instruments. In Example 4.1, it was unrealistically assumed that exact noise-free versions of the regressors were available for use as instruments. A common way of obtaining instruments in practice is by simulation of an auxiliary model, see for example Thil et al. [2008]. In this simulation study, the model obtained by taking the LS estimate for the parameters was used for this so that

$$
\zeta(k)=\left[\hat{x}^{L S}(k-1)\left|\hat{x}^{L S}(k-1)\right| u(k-1)\right]^{T},
$$

where

$$
\begin{aligned}
\hat{x}^{L S}(k+1) & =\hat{n}_{N}^{L S} \hat{x}^{L S}(k)\left|\hat{x}^{L S}(k)\right|+\hat{f}_{N}^{L S} \hat{x}^{L S} u(k), \quad k=1, \ldots, N, \\
\hat{x}^{L S}(1) & =0 .
\end{aligned}
$$

The parameters were then refined by iteratively letting the instruments instead be simulated from the model parameterized by the latest version of $\hat{\theta}_{N}^{I V}$ until 
convergence, as described in Young [2008]. In order to obtain zero-mean instruments, the average value of each component of $\zeta(k)$ was simply subtracted

$$
\tilde{\zeta}_{i}(k)=\zeta_{i}(k)-\frac{1}{N} \sum_{k=1}^{N} \zeta_{i}(k), i=1,2 .
$$

The first two sets of simulations were based on different experiment designs. In both these sets, the non-additive disturbance had zero mean, $\bar{v}=0$, and the predictor model

$$
\hat{y}(k \mid \theta)=\left[y_{1}(k-1)\left|y_{1}(k-1)\right| u(k-1)\right]\left[\begin{array}{l}
n \\
f
\end{array}\right],
$$

was considered. The baseline input, $\tilde{u}(k)$, followed a zero-mean Gaussian distribution with unit variance $\tilde{u}(k) \sim \mathcal{N}(0,1)$. In the first set of simulations, this input was used right away $u(k)=\tilde{u}(k)$, which means that the input had zero mean. Histograms showing the parameter errors for the estimators are provided in Figures 4.1 and 4.2. The IV estimators coincide because the input has zero mean and the model which is used to simulate the instruments is based on an odd function. Neither the LS nor the IV estimators seems to be consistent in this setup.

In the second set of simulations, the input was modified as

$$
u(k)= \begin{cases}|\tilde{u}(k)|, & \text { if }|\tilde{u}(k)|>0.6 \\ 0.6, & \text { otherwise. }\end{cases}
$$

in order to have the system state well-separated from the origin. Histograms showing the parameter errors for the three estimators are provided in Figures 4.3, 4.4 and 4.5. In this case, the zero-mean IV estimator seems consistent.

After this, two sets of simulations were performed with $\bar{v}=0.1$ and the input (4.65). First, the predictor model (4.64) was considered. The estimation errors are then given in Figures 4.6, 4.7 and 4.8, where it can be observed that none of the estimators seem consistent.

In the fourth set of simulations, the supplementary measurements were utilized by forming the predictor

$$
\hat{y}_{r}(k \mid \theta)==[\check{y}(k-1)|\check{y}(k-1)| u(k-1)]\left[\begin{array}{l}
n \\
f
\end{array}\right],
$$

where $\check{y}(k)=y_{1}(k)+y_{2}(k)$. The corresponding estimation errors are presented in Figures $4.9,4.10$ and 4.11 . In this case, the zero-mean IV estimator, $\hat{\theta}_{N}^{I V_{2}}$, seems consistent. 

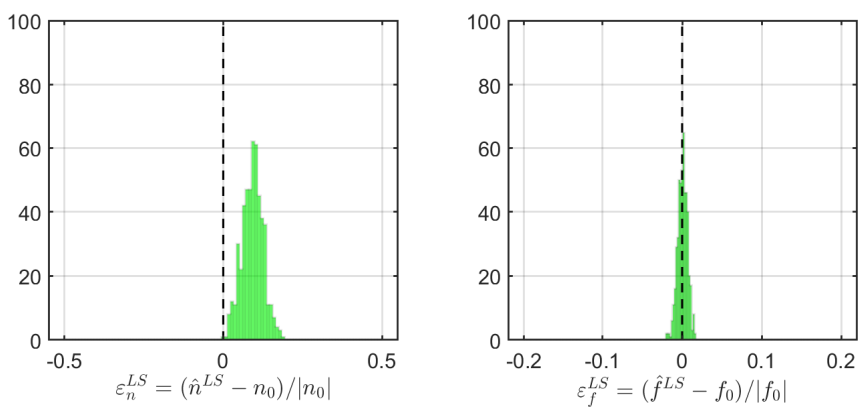

Figure 4.1: Normalized LS estimation errors for the set of Monte Carlo runs with $\bar{v}=0$ and zero-mean input. The mean errors plus/minus one standard deviation are $\varepsilon_{\hat{n}}^{L S}=0.0922 \pm 0.0330, \varepsilon_{\hat{f}}^{L S}=0.0005 \pm 0.0062$.
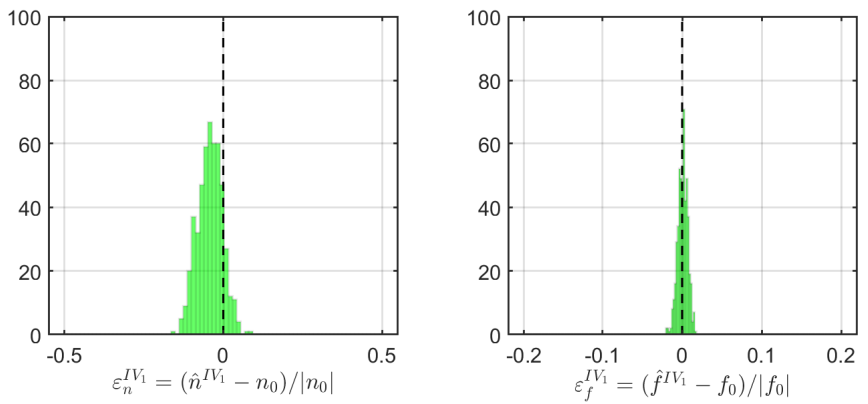

Figure 4.2: Normalized IV estimation errors for the set of Monte Carlo runs with $\bar{v}=0$ and zero-mean input. The mean errors plus/minus one standard deviation are $\varepsilon_{\hat{n}}^{I V_{1}}=-0.0423 \pm 0.0389, \varepsilon_{\hat{f}}^{I V_{1}}=0.0007 \pm 0.0062$. 

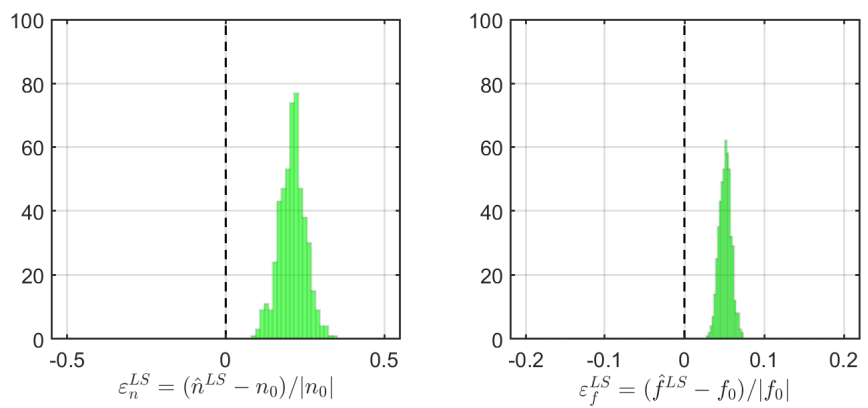

Figure 4.3: Normalized LS estimation errors for the set of Monte Carlo runs with $\bar{v}=0$ and input according to (4.65). The mean errors plus/minus one standard deviation are $\varepsilon_{\hat{n}}=0.2097 \pm 0.0416, \varepsilon_{\hat{f}}^{L S}=0.0511 \pm 0.0076$.
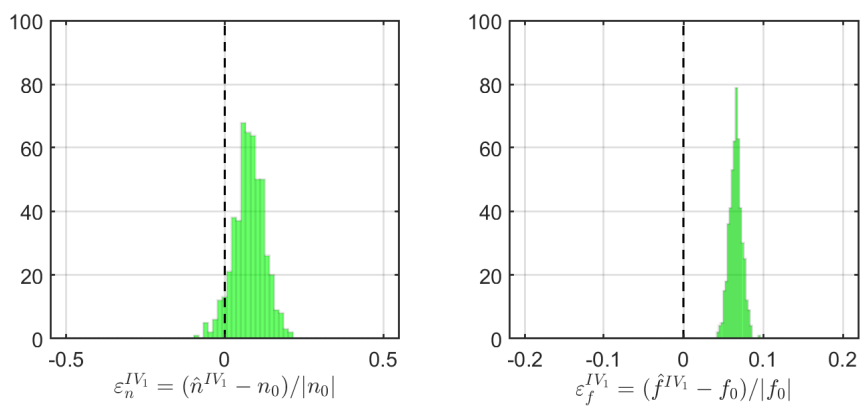

Figure 4.4: Normalized IV estimation errors for the set of Monte Carlo runs with $\bar{v}=0$ and input according to (4.65). The mean errors plus/minus one standard deviation are $\varepsilon_{\hat{n}}^{I V_{1}}=0.0762 \pm 0.0486, \varepsilon_{\hat{f}}^{I V_{1}}=0.0654 \pm 0.0079$.
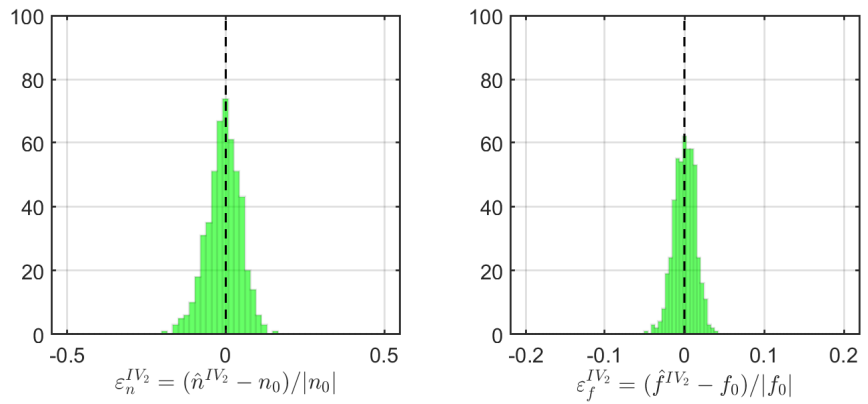

Figure 4.5: Normalized zero-mean-instrument IV estimation errors for the set of Monte Carlo runs with $\bar{v}=0$ and input according to (4.65). The mean errors plus/minus one standard deviation are $\varepsilon_{\hat{n}}^{I V_{2}}=-0.0066 \pm 0.0531$, $\varepsilon_{\hat{f}}^{I V_{2}}=0.0002 \pm 0.0137$. 

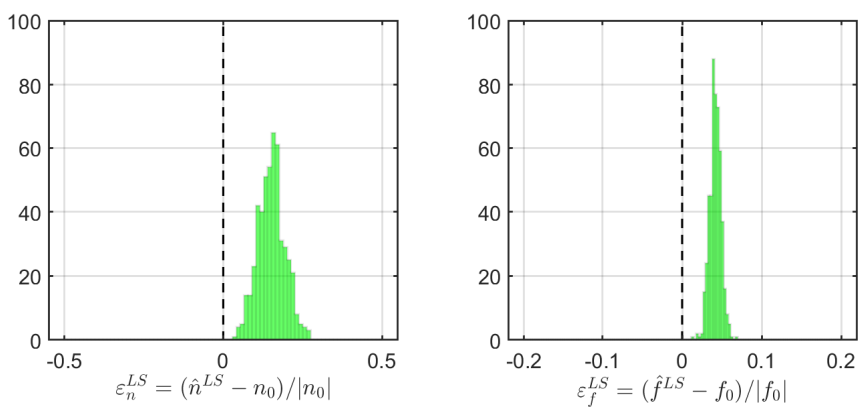

Figure 4.6: Normalized LS estimation errors for the set of Monte Carlo runs with $\bar{v}=0.1$, the predictor model (4.64) and input according to (4.65). The mean errors plus/minus one standard deviation are $\varepsilon_{\hat{n}}^{L S}=0.1511 \pm 0.0428$, $\varepsilon_{\hat{f}}=0.0417 \pm 0.0073$.
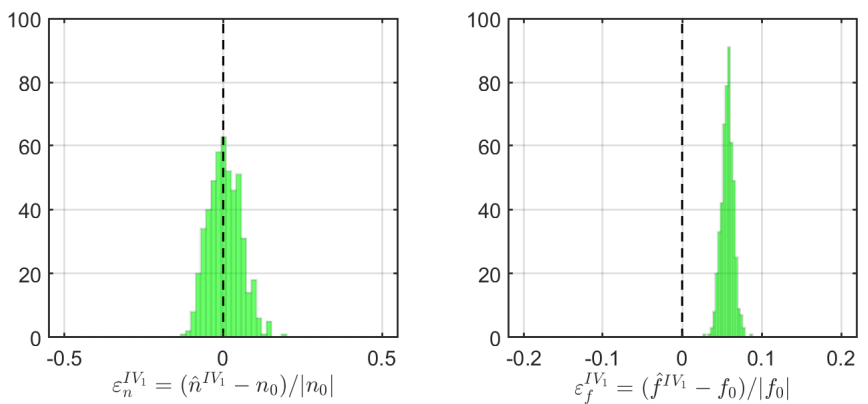

Figure 4.7: Normalized IV estimation errors for the set of Monte Carlo runs with $\bar{v}=0.1$, the predictor model (4.64) and input according to (4.65). The mean errors plus/minus one standard deviation are $\varepsilon_{\hat{n}}^{I V_{1}}=0.0051 \pm 0.0505$, $\varepsilon_{\hat{f}}^{I V_{1}}=0.0568 \pm 0.0077$.
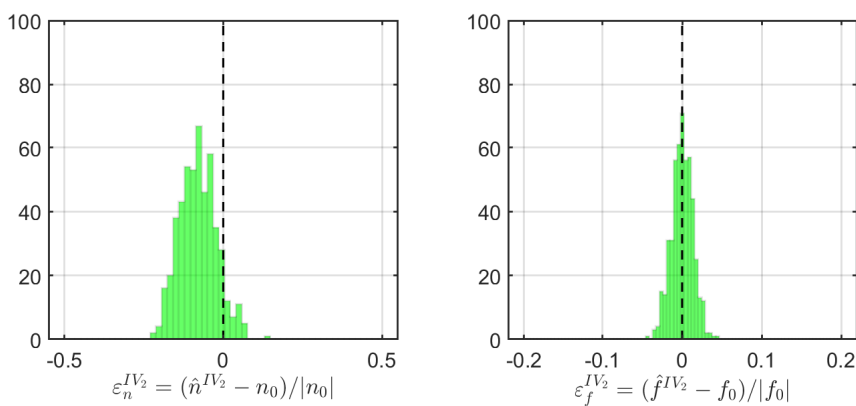

Figure 4.8: Normalized zero-mean-instrument IV estimation errors for the set of Monte Carlo runs with $\bar{v}=0.1$, the predictor model (4.64) and input according to (4.65). The mean errors plus/minus one standard deviation are $\varepsilon_{\hat{n}}^{I V_{2}}=-0.0798 \pm 0.0578, \varepsilon_{\hat{f}}^{I V_{2}}=-0.0001 \pm 0.0135$. 

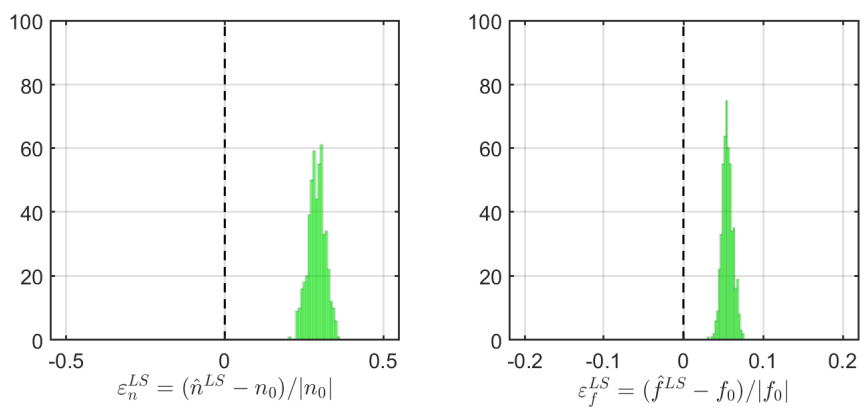

Figure 4.9: Normalized LS estimation errors for the set of Monte Carlo runs with $\bar{v}=0.1$, the predictor model (4.66) and input according to (4.65). The mean errors plus/minus one standard deviation are $\varepsilon_{\hat{n}}^{L S}=0.2899 \pm 0.0270$, $\varepsilon_{\hat{f}}=0.0552 \pm 0.0069$.
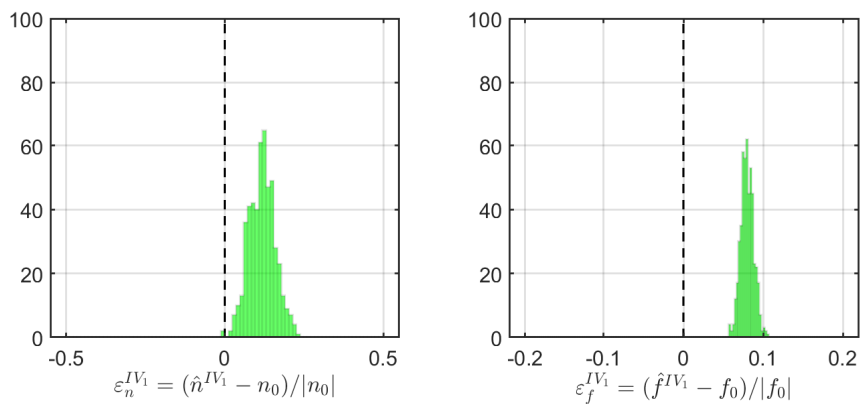

Figure 4.10: Normalized IV estimation errors for the set of Monte Carlo runs with $\bar{v}=0.1$, the predictor model (4.66) and input according to (4.65). The mean errors plus/minus one standard deviation are $\varepsilon_{\hat{n}}^{I V_{1}}=0.1168 \pm 0.0409$, $\varepsilon_{\hat{f}}^{I V_{1}}=0.0800 \pm 0.0082$.
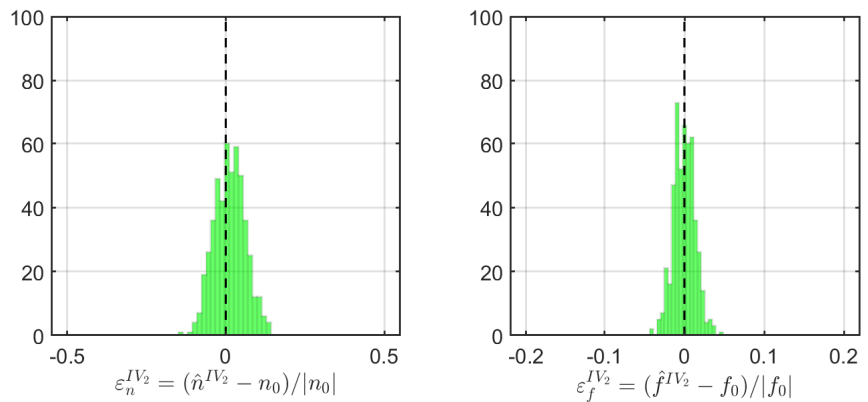

Figure 4.11: Normalized zero-mean-instrument IV estimation errors for the set of Monte Carlo runs with $\bar{v}=0.1$, the predictor model (4.66) and input according to (4.65). The mean errors plus/minus one standard deviation are $\varepsilon_{\hat{n}}^{I V_{2}}=0.0122 \pm 0.0486, \varepsilon_{\hat{f}}^{I V_{2}}=-0.0003 \pm 0.0133$. 


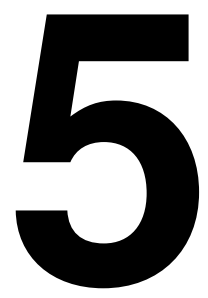

\section{Estimating Disturbances}

In the previous chapter, a way to get consistency in the more general case where the non-additive disturbance has non-zero mean, was shown to be to add a second sensor for measuring the disturbance. In practice it is not always desired, or even possible, to add additional sensors. In this chapter, another method of obtaining consistent parameter estimators in the case of non-zero mean disturbances is explored. The main idea is to augment the regression vector with elements that capture the behavior of the noise distribution, i.e. to estimate the first and second-order moments of the disturbances alongside the model parameters.

It will be assumed that data is generated based on a second-order modulus maneuvering system

$$
\begin{aligned}
v(k+1) & =f\left(v(k), v_{r}(k), v_{q}(k), \tilde{\tau}(k)\right), \\
\mathbf{y}(k) & =\left[\begin{array}{l}
y_{u}(k) \\
y_{v}(k) \\
y_{r}(k) \\
y_{\psi}(k)
\end{array}\right]=\left[\begin{array}{c}
u(k) \\
v(k) \\
r(k) \\
\psi(k)
\end{array}\right]+\left[\begin{array}{c}
e_{u}(k) \\
e_{v}(k) \\
e_{r}(k) \\
e_{\psi}(k)
\end{array}\right],
\end{aligned}
$$

where in addition to the velocity states, the heading angle, $\psi$, is assumed to be measured. Recall from Chapter 3 that $v_{r}=v-v_{c}$ and $v_{q}=v-v_{w}$, where $v_{c}$ is the velocity of an ocean current and $v_{w}$ is the wind velocity. These disturbances are assumed to be stationary stochastic processes in the $n$-frame, whereas in the body-fixed frame they depend on the attitude of the ship

$$
v_{c}(k)=R^{-1}(\psi(k)) v_{c, n}(k)=\left[\begin{array}{ccc}
\cos (\psi(k)) & \sin (\psi(k)) & 0 \\
\sin (\psi(k)) & \cos (\psi(k)) & 0 \\
0 & 0 & 1
\end{array}\right]\left[\begin{array}{c}
v_{c, N S}(k) \\
v_{c, E W}(k) \\
0
\end{array}\right]
$$




$$
v_{w}(k)=R^{-1}(\psi(k)) v_{w, n}(k)=\left[\begin{array}{ccc}
\cos (\psi(k)) & \sin (\psi(k)) & 0 \\
-\sin (\psi(k)) & \cos (\psi(k)) & 0 \\
0 & 0 & 1
\end{array}\right]\left[\begin{array}{c}
v_{w, N S}(k) \\
v_{w, E W}(k) \\
0
\end{array}\right] .
$$

The north/south component, $v_{., N S}(k)$, is positive for southern winds and the east/west component, $v_{., E W}(k)$, is positive for western winds, i.e. winds going from south to north and winds going from west to east, respectively. The measurement noises, $e_{u}(k), e_{v}(k), e_{r}(k)$, and $e_{\psi}(k)$, are assumed to follow stationary zero-symmetric distributions and be bounded in magnitude

$$
\begin{gathered}
-\eta_{e_{u}}<e_{u}(k)<\eta_{e_{u}}, \\
-\eta_{e_{v}}<e_{v}(k)<\eta_{e_{v}}, \\
-\eta_{e_{r}}<e_{r}(k)<\eta_{e_{r}}, \\
-\eta_{e_{\psi}}<e_{\psi}(k)<\eta_{e_{\psi}},
\end{gathered}
$$

whereas the environmental disturbances are assumed to have a deterministic part which is possibly non-zero and a stochastic part with zero mean which is bounded in magnitude

$$
\begin{array}{r}
v_{c, N S}(k)=\bar{v}_{c, N S}+\tilde{v}_{c, N S}(k), \quad-\eta_{\tilde{v}_{c}}<\tilde{v}_{c, N S}(k)<\eta_{\tilde{v}_{c}}, \\
v_{c, E W}(k)=\bar{v}_{c, E W}+\tilde{v}_{c, E W}(k), \quad-\eta_{\tilde{v}_{c}}<\tilde{v}_{c, E W}(k)<\eta_{\tilde{v}_{c}}, \\
v_{w, N S}(k)=\bar{v}_{w, N S}+\tilde{v}_{w, N S}(k), \quad-\eta_{\tilde{v}_{w}}<\tilde{v}_{w, N S}(k)<\eta_{\tilde{v}_{w}}, \\
v_{w, E W}(k)=\bar{v}_{w, E W}+\tilde{v}_{w, E W}(k), \quad-\eta_{\tilde{v}_{w}}<\tilde{v}_{w, E W}(k)<\eta_{\tilde{v}_{w}} .
\end{array}
$$

Moreover, it will be assumed that the measurement noises are independent, both mutually and of the environmental disturbances.

The ideas of this chapter are first presented in Section 5.1 by use of a simple SISO system. The fact that this system is simple makes it transparent for analysis. The ideas are then shown to be generalizable to estimation of a maneuvering model in Section 5.2.

\subsection{1-DOF model}

In Chapter 3, the simple surge model (3.56) was derived. Assume that data is generated based on that model and that the absolute surge speed and the yaw angle are measured

$$
\begin{aligned}
u(k+1) & =u(k)+\mathcal{X}_{u} u_{r}(k)+\mathcal{X}_{|u| u}\left|u_{r}(k)\right| u_{r}(k)+\mathcal{W}_{|u| u \mid}\left|u_{q}(k)\right| u_{q}(k)+\mathcal{X}_{\mu} \tilde{\tau}_{x}(k), \\
y_{u}(k) & =u(k)+e_{u}(k), \\
y_{\psi}(k) & =\psi(k)+e_{\psi}(k) .
\end{aligned}
$$

Annotate the parameters required to perform undisturbed simulations of system (5.6) as $\theta_{0}=\left[\begin{array}{lll}1+\mathcal{X}_{u} & \mathcal{X}_{|u| u}+\mathcal{W}_{|u| u} & \mathcal{X}_{\mu}\end{array}\right]^{T}$. Note that for performing undisturbed 
simulations, it is not required to uniquely identify the hydrodynamic and aerodynamic damping as separate effects, but solely an estimate of the aggregation $\mathcal{X}_{|u| u}+\mathcal{W}_{|u| u}$ is needed. Moreover, let $\theta_{0}^{*}=\left[\begin{array}{llll}1+\mathcal{X}_{u} & \mathcal{X}_{|u| u} & \mathcal{W}_{|u| u} & \mathcal{X}_{\mu}\end{array}\right]^{T}$ denote a parameter vector where the two damping effects are separated.

Assume that experiments are performed in a way such that $u_{r}(k)>0, u_{q}(k)>0$ and $u(k)+e_{u}(k)>0$. By the previous assumptions, (5.4) and (5.5), a sufficient condition for fulfilling this is

$$
u(k)>\max \left(\eta_{e_{u}}, \bar{v}_{c, N S}+\eta_{\tilde{v}_{c}}, \bar{v}_{c, E W}+\eta_{\tilde{v}_{c}}, \bar{v}_{w, N S}+\eta_{\tilde{v}_{w}}, \bar{v}_{w, E W}+\eta_{\tilde{v}_{w}}\right) .
$$

Here, it was utilized that $|\sin (\psi(k))| \leq 1$ and $|\cos (\psi(k))| \leq 1$. Under assumption of an experiment design like (5.7), (5.6a) may be rewritten as

$$
\begin{aligned}
u(k+1) & =u(k)+\mathcal{X}_{u}\left(u(k)-u_{c}(k)\right)+\mathcal{X}_{|u| u}\left(u(k)-u_{c}(k)\right)^{2}+\mathcal{W}_{|u| u}\left(u(k)-u_{w}(k)\right)^{2} \\
& +\mathcal{X}_{\mu} \tilde{\tau}_{x}(k)=\left(1+\mathcal{X}_{u}-2 \mathcal{X}_{|u| u} u_{c}(k)-2 \mathcal{W}_{|u| u} u_{w}(k)\right) u(k) \\
& +\left(\mathcal{X}_{|u| u}+\mathcal{W}_{|u| u}\right) u(k)^{2}+\mathcal{X}_{\mu} \tilde{\tau}_{x}(k)-\mathcal{X}_{u} u_{c}(k)+\mathcal{X}_{|u| u} u_{c}(k)^{2}+\mathcal{W}_{|u| u} u_{w}(k)^{2}
\end{aligned}
$$

\subsubsection{Straight-line path motion}

First, consider a predictor that only utilizes the surge measurement

$$
\hat{y}_{u, 1}(k \mid \theta)=\left[\begin{array}{ll}
y_{u}(k-1) & y_{u}(k-1)\left|y_{u}(k-1)\right| \quad \tilde{\tau}_{x}(k-1)
\end{array}\right]\left[\begin{array}{c}
\theta_{1} \\
\theta_{2} \\
\theta_{3}
\end{array}\right] \triangleq \varphi_{u, 1}^{T}(k) \theta,
$$

and assume that the ship is moving on a straight-line path

$$
\psi(k)=\bar{\psi}, k=1, \ldots, N .
$$

In this case, the attitude dependence of the environmental disturbances can be ignored

$$
\begin{gathered}
u_{c}(k)=v_{c, N S}(k) \cos (\bar{\psi})+v_{c, E W}(k) \sin (\bar{\psi}) \triangleq u_{c, \bar{\psi}}(k), \\
u_{w}(k)=v_{w, N S}(k) \cos (\bar{\psi})+v_{w, E W}(k) \sin (\bar{\psi}) \triangleq u_{w, \bar{\psi}}(k) .
\end{gathered}
$$

Assume that there exists an instrument vector $\zeta_{u, 1}(k)$ that is independent of $u_{c, \bar{\psi}}(k), u_{w, \bar{\psi}}(k), e_{u}(k)$ and has zero mean, $\bar{E}\left\{\zeta_{u, 1}(k)\right\}=0$. Also, assume that the input is informative so that (2.19a) holds. By the IV equation, (2.20), the parameters of an IV estimator will converge to the values that make

$$
\bar{E}\left\{\zeta_{u, 1}(k)\left(y_{u}(k)-\varphi_{u, 1}^{T}(k) \theta\right)\right\}=0
$$


This expectation written out explicitly is

$$
\begin{aligned}
\bar{E} & \left\{\zeta _ { u , 1 } ( k ) \left(\left(1+\mathcal{X}_{u}-2 \mathcal{X}_{|u| u} u_{c, \bar{\psi}}(k-1)-2 \mathcal{W}_{|u| u} u_{w, \bar{\psi}}(k-1)\right) u(k-1)+\left(\mathcal{X}_{|u| u}\right.\right.\right. \\
& \left.+\mathcal{W}_{|u| u}\right) u(k-1)^{2}+\mathcal{X}_{\mu} \tilde{\tau}_{x}(k-1)-\mathcal{X}_{u} u_{c, \bar{\psi}}(k-1)+\mathcal{X}_{|u| u} u_{c, \bar{\psi}}(k-1)^{2} \\
& +\mathcal{W}_{|u| u} u_{w, \bar{\psi}}(k-1)^{2}+e_{u}(k)-\theta_{1}\left(u(k-1)+e_{u}(k-1)\right)-\theta_{2}\left(u(k-1)^{2}\right. \\
& \left.\left.\left.+2 u(k-1) e_{u}(k-1)+e_{u}(k-1)^{2}\right)-\theta_{3} \tilde{\tau}_{x}(k-1)\right)\right\} \\
& =\left(1+\mathcal{X}_{u}-2 \mathcal{X}_{|u| u} \bar{u}_{c, \bar{\psi}}-2 \mathcal{W}_{|u| u} \bar{u}_{w, \bar{\psi}}-\theta_{1}\right) \bar{E}\left\{\zeta_{u, 1}(k) u(k-1)\right\} \\
& +\left(\mathcal{X}_{|u| u}+\mathcal{W}_{|u| u}-\theta_{2}\right) \bar{E}\left\{\zeta_{u, 1}(k) u(k-1)^{2}\right\}+\left(\mathcal{X}_{\mu}-\theta_{3}\right) \bar{E}\left\{\zeta_{u, 1}(k) \tilde{\tau}_{x}(k-1)\right\},
\end{aligned}
$$

where

$$
\begin{gathered}
\bar{u}_{c, \bar{\psi}}=\bar{E}\left\{u_{c}(k-1)\right\}=\bar{v}_{c, N S} \cos (\bar{\psi})+\bar{v}_{c, E W} \sin (\bar{\psi}), \\
\bar{u}_{w, \bar{\psi}}=\bar{E}\left\{u_{w}(k-1)\right\}=\bar{v}_{w, N S} \cos (\bar{\psi})+\bar{v}_{w, E W} \sin (\bar{\psi}) .
\end{gathered}
$$

From (5.13) it can be seen that the asymptotic parameter estimates are

$$
\lim _{N \rightarrow \infty} \hat{\theta}_{N}^{I V}=\left[\begin{array}{c}
1+\mathcal{X}_{u}-2 \mathcal{X}_{|u| u} \bar{u}_{c, \bar{\psi}}-2 \mathcal{W}_{|u| u} \bar{u}_{w, \bar{\psi}} \\
\mathcal{X}_{|u| u}+\mathcal{W}_{|u| u} \\
\mathcal{X}_{\mu}
\end{array}\right] \neq \theta_{0} .
$$

There is a clear bias in the estimate of the linear damping. The fact that the IV estimator is biased in this scenario is in agreement with Example 4.2.

Now look at a slightly modified setup, where halfway through the ship is rotated half a lap about the $z_{b}$-axis

$$
\psi(k)= \begin{cases}\bar{\psi}, & \text { for } k=1, \ldots, N / 2, \\ \bar{\psi}+\pi, & \text { for } k=N / 2+1, \ldots, N\end{cases}
$$

The only differences in the calculation of the asymptotic parameter estimates in this case are

$$
\begin{gathered}
\bar{u}_{c, \bar{\psi}}=\bar{E}\left\{u_{c}(k-1)\right\}=0, \\
\bar{u}_{w, \bar{\psi}}=\bar{E}\left\{u_{w}(k-1)\right\}=0,
\end{gathered}
$$

which makes the IV estimator consistent

$$
\lim _{N \rightarrow \infty}^{\text {w.p.1 }} \hat{\theta}_{N}^{I V}=\left[\begin{array}{c}
1+\mathcal{X}_{u} \\
\mathcal{X}_{|u| u}+\mathcal{W}_{|u| u} \\
\mathcal{X}_{\mu}
\end{array}\right]=\theta_{0}
$$


It would be convenient if this modified experiment design could be generalized for identification of more complete models. However, the assumption that the instruments are independent of the environmental disturbances' effect in the $b$ frame is a problem. It was shown in Chapter 3 that motion in surge and yaw are coupled. Most instruments that are independent of $\psi(k)$ are therefore also independent of $u(k)$ and the above considered case where the ship is moving on a straight-line path is an exception. If the experiment design was restricted to motion along straight paths it would be impossible to identify cross terms. Therefore, the result of obtaining consistency by an experiment design like (5.16), is in general of limited usefulness for identification of maneuvering models.

\subsubsection{Augmenting the regression vector}

Based on the observation that the estimation bias depends on the average attitude of the ship, a more general way of obtaining consistency will now be illustrated. Modify the regression vector to

$$
\begin{aligned}
& \varphi_{u, 2}(k)=\left[\begin{array}{llll}
y_{u}(k-1) & y_{u}(k-1)\left|y_{u}(k-1)\right| & \tilde{\tau}_{x}(k-1) & \cos \left(y_{\psi}(k-1)\right)
\end{array} \ldots\right. \\
& \sin \left(y_{\psi}(k-1)\right)\left|y_{u}(k-1)\right| \cos \left(y_{\psi}(k-1)\right) \quad\left|y_{u}(k-1)\right| \sin \left(y_{\psi}(k-1)\right) \\
& \operatorname{sgn}\left(\left(y_{u}(k-1)\right) \cos ^{2}\left(y_{\psi}(k-1)\right) \operatorname{sgn}\left(\left(y_{u}(k-1)\right) \sin \left(2 y_{\psi}(k-1)\right)\right]^{T}\right. \text {, }
\end{aligned}
$$

where $\operatorname{sgn}($.$) is the signum function and consider the corresponding predictor$

$$
\hat{y}_{u, 2}(k \mid \theta)=\varphi_{u, 2}^{T}(k) \cdot\left[\begin{array}{lllllllll}
\theta_{1} & \theta_{2} & \theta_{3} & b_{1} & b_{2} & b_{3} & b_{4} & b_{5} & b_{6}
\end{array}\right]^{T} \triangleq \varphi_{u, 2}^{T}(k) \theta .
$$

Notably, the final six elements of $\theta$ are annotated differently. What they have in common is that they are not needed for performing undisturbed simulations of system (5.6). The main reason for including their corresponding terms in the model is to obtain more accurate estimates of the first three parameters. Assume that there exists an instrument vector $\zeta_{u, 2}(k) \in \mathbb{R}^{9}$, which is independent of $e_{u}(k)$ and $e_{\psi}(k)$, has zero mean and fulfills (2.19a). Recall that in Chapter 3

$$
\rho_{e_{\psi}}=\bar{E}\left\{\cos \left(e_{\psi}(k-1)\right)\right\}
$$

was defined. Now also let

$$
\sigma_{e_{\psi}} \triangleq \bar{E}\left\{\cos ^{2}\left(e_{\psi}(k-1)\right)\right\}
$$


By use of trigonometric identities and the assumptions that $e_{\psi}(k)$ follows a zerosymmetric distribution and $\bar{E}\left\{\zeta_{u, 2}(k)\right\}=0$, it can be observed that

$$
\begin{aligned}
& \bar{E}\left\{\zeta_{u, 2}(k) \sin \left(2 y_{\psi}(k-1)\right)\right\}=\bar{E}\left\{\zeta _ { u , 2 } ( k ) \left(\sin (2 \psi(k-1)) \cos \left(2 e_{\psi}(k-1)\right)\right.\right. \\
& \left.\left.+\cos (2 \psi(k-1)) \sin \left(2 e_{\psi}(k-1)\right)\right)\right\}=\bar{E}\left\{\zeta_{u, 2}(k) \sin (2 \psi(k-1))\right\} \bar{E}\left\{\cos \left(2 e_{\psi}(k-1)\right)\right\} \\
& =\bar{E}\left\{\zeta_{u, 2}(k) \sin (2 \psi(k-1))\right\} \bar{E}\left\{\cos ^{2}\left(e_{\psi}(k-1)\right)-\sin ^{2}\left(e_{\psi}(k-1)\right)\right\} \\
& =\left(2 \sigma_{e_{\psi}}-1\right) \bar{E}\left\{\zeta_{u, 2}(k) \sin (2 \psi(k-1))\right\}, \\
& \bar{E}\left\{\zeta_{u, 2}(k) \cos ^{2}\left(y_{\psi}(k-1)\right)\right\}=\frac{1}{2} \bar{E}\left\{\zeta_{u, 2}(k)\left(1+\cos \left(2 y_{\psi}(k-1)\right)\right)\right\} \\
& =\frac{1}{2} \bar{E}\left\{\zeta_{u, 2}(k)\left(\cos (2 \psi(k-1)) \cos \left(2 e_{\psi}(k-1)\right)-\sin (2 \psi(k-1)) \sin \left(2 e_{\psi}(k-1)\right)\right)\right\} \\
& =\frac{1}{2} \bar{E}\left\{\zeta_{u, 2}(k) \cos (2 \psi(k-1))\right\} \bar{E}\left\{\cos \left(2 e_{\psi}(k-1)\right)\right\} \\
& =\left(2 \sigma_{e_{\psi}}-1\right) \bar{E}\left\{\zeta_{u, 2}(k) \cos ^{2}(\psi(k-1))\right\}
\end{aligned}
$$

and also that

$$
\begin{aligned}
\bar{E}\left\{\zeta_{u, 2}(k) \sin ^{2}\left(y_{\psi}(k-1)\right)\right\} & =\bar{E}\left\{\zeta_{u, 2}(k)\left(1-\cos ^{2}\left(y_{\psi}(k-1)\right)\right)\right\} \\
& =-\bar{E}\left\{\zeta_{u, 2}(k) \cos ^{2}\left(y_{\psi}(k-1)\right)\right\} .
\end{aligned}
$$

For analyzing the convergence of an IV estimator, again the IV equation, (2.20), can be studied. Moreover, again assume that the experiments are performed such that (5.7) is fulfilled and recall that this implies that the surge-speed measurements are positive. Using (5.23), (5.24) and (5.25), the left-hand side of the IV equation can in this case be expressed explicitly as

$$
\begin{aligned}
& \bar{E}\left\{\zeta_{u, 2}(k)\left(y_{u}(k)-\varphi_{u, 2}^{T}(k) \theta\right)\right\}=\bar{E}\left\{\zeta _ { u , 2 } ( k ) \left[\left(1+\mathcal{X}_{u}-2 \mathcal{X}_{|u| u}\left(v_{c, N S}(k-1)\right.\right.\right.\right. \\
& \left.\cdot \cos (\psi(k-1))+v_{c, E W}(k-1) \sin (\psi(k-1))\right)-2 \mathcal{W}_{|u| u}\left(v_{w, N S}(k-1) \cos (\psi(k-1))\right. \\
& \left.\left.+v_{w, E W}(k-1) \sin (\psi(k-1))\right)\right) u(k-1)+\left(\mathcal{X}_{|u| u}+\mathcal{W}_{|u| u}\right) u(k-1)^{2}+\mathcal{X}_{\mu} \tilde{\tau}_{x}(k-1) \\
& -\mathcal{X}_{u}\left(v_{c, N S}(k-1) \cos (\psi(k-1))+v_{c, E W}(k-1) \sin (\psi(k-1))\right)+\mathcal{X}_{|u| u}\left(v_{c, N S}(k)^{2}\right. \\
& \cdot \cos ^{2}(\psi(k-1))+2 v_{c, N S}(k-1) v_{c, E W}(k-1) \cos (\psi(k-1)) \sin (\psi(k-1))
\end{aligned}
$$




$$
\begin{aligned}
& \left.+v_{c, E W}(k-1)^{2} \sin ^{2}(\psi(k-1))\right)+\mathcal{W}_{|u| u}\left(v_{w, N S}(k)^{2} \cos ^{2}(\psi(k-1))+2 v_{w, N S}(k-1)\right. \\
& \left.\cdot v_{w, E W}(k-1) \cos (\psi(k-1)) \sin (\psi(k-1))+v_{w, E W}(k-1)^{2} \sin ^{2}(\psi(k-1))\right) \\
& +e_{u}(k)-\theta_{1}\left(u(k-1)+e_{u}(k-1)\right)-\theta_{2}\left(u(k-1)^{2}+2 u(k-1) e_{u}(k-1)\right. \\
& \left.+e_{u}(k-1)^{2}\right)-\theta_{3} \tilde{\tau}_{x}(k-1)-b_{1} \cos \left(\psi(k-1)+e_{\psi}(k-1)\right) \\
& -b_{2} \sin \left(\psi(k-1)+e_{\psi}(k-1)\right)-b_{3}\left(u(k-1)+e_{u}(k-1)\right) \cos \left(\psi(k-1)+e_{\psi}(k-1)\right) \\
& -b_{4}\left(u(k-1)+e_{u}(k-1)\right) \sin \left(\psi(k-1)+e_{\psi}(k-1)\right)-b_{5} \cos ^{2}\left(\psi(k-1)+e_{\psi}(k-1)\right) \\
& \left.\left.-b_{6} \sin \left(2\left(\psi(k-1)+e_{\psi}(k-1)\right)\right)\right]\right\}=\left(1+\mathcal{X}_{u}-\theta_{1}\right) \bar{E}\left\{\zeta_{u, 2}(k) u(k-1)\right\} \\
& +\left(\mathcal{X}_{|u| u}+\mathcal{W}_{|u| u}-\theta_{2}\right) \bar{E}\left\{\zeta_{u, 2}(k) u(k-1)^{2}\right\}+\left(\mathcal{X}_{\mu}-\theta_{3}\right) \bar{E}\left\{\zeta_{u, 2}(k) \tilde{\tau}_{x}(k-1)\right\} \\
& +\left(-\mathcal{X}_{u} \bar{v}_{c, N S}-\rho_{e_{\psi}} b_{1}\right) \bar{E}\left\{\zeta_{u, 2}(k) \cos (\psi(k-1))\right\} \\
& +\left(-\mathcal{X}_{u} \bar{v}_{c, E W}-\rho_{e_{\psi}} b_{2}\right) \bar{E}\left\{\zeta_{u, 2}(k) \sin (\psi(k-1))\right\} \\
& +\left(-2 \mathcal{X}_{|u| u} \bar{v}_{c, N S}-2 \mathcal{W}_{|u| u} \bar{v}_{w, N S}-\rho_{e_{\psi}} b_{3}\right) \bar{E}\left\{\zeta_{u, 2}(k) \cos (\psi(k-1)) u(k-1)\right\} \\
& +\left(-2 \mathcal{X}_{|u| u} \bar{v}_{c, E W}-2 \mathcal{W}_{|u| u} \bar{v}_{w, E W}-\rho_{e_{\psi}} b_{4}\right) \bar{E}\left\{\zeta_{u, 2}(k) \sin (\psi(k-1)) u(k-1)\right\} \\
& +\left(\mathcal{X}_{|u| u}\left(\bar{E}\left\{v_{c, N S}(k-1)^{2}\right\}-\bar{E}\left\{v_{c, E W}(k-1)^{2}\right\}\right)+\mathcal{W}_{|u| u}\left(\bar{E}\left\{v_{w, N S}(k-1)^{2}\right\}\right.\right. \\
& \left.\left.-\bar{E}\left\{v_{w, E W}(k-1)^{2}\right\}\right)-\left(2 \sigma_{e_{\psi}}-1\right) b_{5}\right) \bar{E}\left\{\zeta_{u, 2}(k) \cos ^{2}(\psi(k-1))\right\} \\
& +\left(\mathcal{X}_{|u| u} \bar{E}\left\{v_{c, N S}(k-1) v_{c, E W}(k-1)\right\}+\mathcal{W}_{|u| u} \bar{E}\left\{v_{w, N S}(k-1) v_{w, E W}(k-1)\right\}\right. \\
& \left.-\left(2 \sigma_{e_{\psi}}-1\right) b_{6}\right) \bar{E}\left\{\zeta_{u, 2}(k) \sin (2 \psi(k-1))\right\} \text {. }
\end{aligned}
$$

From this it can be seen that the asymptotic parameter estimates are

$$
\lim _{N \rightarrow \infty}^{\text {w.p.1 }} \hat{\theta}_{N}^{I V}=\left[\begin{array}{c}
1+\mathcal{X}_{u} \\
\mathcal{X}_{|u| u}+\mathcal{W}_{|u| u} \\
\mathcal{X}_{\mu} \\
-\frac{1}{\rho_{e_{\psi}}} \mathcal{X}_{u} \bar{v}_{c, N S} \\
-\frac{1}{\rho_{e_{\psi}}} \mathcal{X}_{u} \bar{v}_{c, E W} \\
\frac{1}{\rho_{e_{\psi}}}\left(-2 \mathcal{X}_{|u| u} \bar{v}_{c, N S}-2 \mathcal{W}_{|u| u} \bar{v}_{w, N S}\right) \\
\frac{1}{\rho_{e_{\psi}}}\left(-2 \mathcal{X}_{|u| u} \bar{v}_{c, E W}-2 \mathcal{W}_{|u| u} \bar{v}_{w, E W}\right) \\
\frac{1}{2 \sigma_{e_{\psi}}-1} \xi_{1}\left(v_{c, n}, v_{w, n}\right) \\
\frac{1}{2 \sigma_{e_{\psi}}-1} \xi_{2}\left(v_{c, n}, v_{w, n}\right)
\end{array}\right]=\left[\begin{array}{c}
\theta_{0} \\
\sim
\end{array}\right] \text {, }
$$


where

$$
\begin{aligned}
& \xi_{1}\left(v_{c, n}, v_{w, n}\right)= \mathcal{X}_{|u| u}\left(\bar{E}\left\{v_{c, N S}(k-1)^{2}\right\}-\bar{E}\left\{v_{c, E W}(k-1)^{2}\right\}\right) \\
&+\mathcal{W}_{|u| u}\left(\bar{E}\left\{v_{w, N S}(k-1)^{2}\right\}-\bar{E}\left\{v_{w, E W}(k-1)^{2}\right\}\right), \\
& \xi_{2}\left(v_{c, n}, v_{w, n}\right)=\mathcal{X}_{|u| u} \bar{E}\left\{v_{c, N S}(k-1) v_{c, E W}(k-1)\right\} \\
&+\mathcal{W}_{|u| u} \bar{E}\left\{v_{w, N S}(k-1) v_{w, E W}(k-1)\right\},
\end{aligned}
$$

depend on the second-order moments of the environmental disturbances. To begin with, this is a consistent estimator of $\theta_{0}$. In addition, the estimator provides information about the first and second-order moments of the environmental disturbances. The first element in $\hat{\theta}_{N}^{I V}$ can be used together with the fourth and fifth element to form

$$
\begin{gathered}
\hat{\bar{v}}_{c, N S}=\frac{\left[\hat{\theta}_{N}^{I V}\right]_{4}}{1-\left[\hat{\theta}_{N}^{I V}\right]_{1}} \rightarrow \frac{1}{\rho_{e_{\psi}}} \bar{v}_{c, N S} \text { w.p. } 1 \text { as } N \rightarrow \infty, \\
\hat{\bar{v}}_{c, E W}=\frac{\left[\hat{\theta}_{N}^{I V}\right]_{5}}{1-\left[\hat{\theta}_{N}^{I V}\right]_{1}} \rightarrow \frac{1}{\rho_{e_{\psi}}} \bar{v}_{c, E W} \text { w.p. } 1 \text { as } N \rightarrow \infty .
\end{gathered}
$$

These disturbance estimates are biased in the same way as the estimates of the linear velocities in Section 3.6. If the yaw angle, $\psi$, is measured with high accuracy, it will be the case that $\rho_{e_{\psi}} \lesssim 1$ and consequently the bias error will be small. In general, the magnitude of $\bar{v}_{c, N S}$ and $\bar{v}_{c, E W}$ will asymptotically be overestimated but since the scale error is the same for both estimates the ratio between them will be correct.

There are too many unknowns to work out independent estimates of $\bar{v}_{w, N S}$ and $\bar{v}_{w, E W}$. However, the sixth and seventh elements of $\hat{\theta}_{N}^{I V}$ give information about the first-order moment of the combined effect of the ocean current and the wind.

\subsubsection{Violating the experiment condition}

It is relevant to study what happens when the experiment assumptions underlying (5.7) are not met. First, consider the completely opposite condition

$$
u(k)<-\max \left(\eta_{e_{u}}, \bar{v}_{c, N S}+\eta_{\tilde{v}_{c}}, \bar{v}_{c, E W}+\eta_{\tilde{v}_{c}}, \bar{v}_{w, N S}+\eta_{\tilde{v}_{w}}, \bar{v}_{w, E W}+\eta_{\tilde{v}_{w}}\right) .
$$

Fulfilling this implies that $u_{r}(k)<0$ and $u_{q}(k)<0$, and in this case (5.6a) may be rewritten as

$$
\begin{aligned}
u(k+1) & =u(k)+\mathcal{X}_{u}\left(u(k)-u_{c}(k)\right)-\mathcal{X}_{|u| u}\left(u(k)-u_{c}(k)\right)^{2}-\mathcal{W}_{|u| u}\left(u(k)-u_{w}(k)\right)^{2} \\
& +\mathcal{X}_{\mu} \tilde{\tau}_{x}(k)=\left(1+\mathcal{X}_{u}+2 \mathcal{X}_{|u| u} u_{c}(k)+2 \mathcal{W}_{|u| u} u_{w}(k)\right) u(k) \\
& -\left(\mathcal{X}_{|u| u}+\mathcal{W}_{|u| u}\right) u(k)^{2}+\mathcal{X}_{\mu} \tilde{\tau}_{x}(k)-\mathcal{X}_{u} u_{c}(k)-\mathcal{X}_{|u| u} u_{c}(k)^{2}-\mathcal{W}_{|u| u} u_{w}(k)^{2}
\end{aligned}
$$


Furthermore, (5.32) implies that $y_{u}(k)<0$ and with the same instrument vector as before, an IV estimator using predictor (5.20) does in this scenario give a lefthand side of the IV equation which can be expressed as

$$
\begin{aligned}
& \bar{E}\left\{\zeta_{u, 2}(k)\left(y_{u}(k)-\varphi_{u, 2}^{T}(k) \theta\right)\right\}=\bar{E}\left\{\zeta _ { u , 2 } ( k ) \left[\left(1+\mathcal{X}_{u}+2 \mathcal{X}_{|u| u}\left(v_{c, N S}(k-1)\right.\right.\right.\right. \\
& \left.\cdot \cos (\psi(k-1))+v_{c, E W}(k-1) \sin (\psi(k-1))\right)+2 \mathcal{W}_{|u| u}\left(v_{w, N S}(k-1) \cos (\psi(k-1))\right. \\
& \left.\left.+v_{w, E W}(k-1) \sin (\psi(k-1))\right)\right) u(k-1)-\left(\mathcal{X}_{|u| u}+\mathcal{W}_{|u| u}\right) u(k-1)^{2}+\mathcal{X}_{\mu} \tilde{\tau}_{x}(k-1) \\
& -\mathcal{X}_{u}\left(v_{c, N S}(k-1) \cos (\psi(k-1))+v_{c, E W}(k-1) \sin (\psi(k-1))\right)-\mathcal{X}_{|u| u}\left(v_{c, N S}(k)^{2}\right. \\
& \cdot \cos ^{2}(\psi(k-1))+2 v_{c, N S}(k-1) v_{c, E W}(k-1) \cos (\psi(k-1)) \sin (\psi(k-1)) \\
& \left.+v_{c, E W}(k-1)^{2} \sin ^{2}(\psi(k-1))\right)-\mathcal{W}_{|u| u}\left(v_{w, N S}(k)^{2} \cos ^{2}(\psi(k-1))+2 v_{w, N S}(k-1)\right. \\
& \left.\cdot v_{w, E W}(k-1) \cos (\psi(k-1)) \sin (\psi(k-1))+v_{w, E W}(k-1)^{2} \sin ^{2}(\psi(k-1))\right) \\
& +e_{u}(k)-\theta_{1}\left(u(k-1)+e_{u}(k-1)\right)+\theta_{2}\left(u(k-1)^{2}+2 u(k-1) e_{u}(k-1)\right. \\
& \left.+e_{u}(k-1)^{2}\right)-\theta_{3} \tilde{\tau}_{x}(k-1)-b_{1} \cos \left(\psi(k-1)+e_{\psi}(k-1)\right) \\
& -b_{2} \sin \left(\psi(k-1)+e_{\psi}(k-1)\right)+b_{3}\left(u(k-1)+e_{u}(k-1)\right) \cos \left(\psi(k-1)+e_{\psi}(k-1)\right) \\
& +b_{4}\left(u(k-1)+e_{u}(k-1)\right) \sin \left(\psi(k-1)+e_{\psi}(k-1)\right)+b_{5} \cos ^{2}\left(\psi(k-1)+e_{\psi}(k-1)\right) \\
& \left.\left.+b_{6} \sin \left(2\left(\psi(k-1)+e_{\psi}(k-1)\right)\right)\right]\right\}=\left(1+\mathcal{X}_{u}-\theta_{1}\right) \bar{E}\left\{\zeta_{u, 2}(k) u(k-1)\right\} \\
& -\left(\mathcal{X}_{|u| u}+\mathcal{W}_{|u| u}-\theta_{2}\right) \bar{E}\left\{\zeta_{u, 2}(k) u(k-1)^{2}\right\}+\left(\mathcal{X}_{\mu}-\theta_{3}\right) \bar{E}\left\{\zeta_{u, 2}(k) \tilde{\tau}_{x}(k-1)\right\} \\
& +\left(-\mathcal{X}_{u} \bar{v}_{c, N S}-\rho_{e_{\psi}} b_{1}\right) \bar{E}\left\{\zeta_{u, 2}(k) \cos (\psi(k-1))\right\} \\
& +\left(-\mathcal{X}_{u} \bar{v}_{c, E W}-\rho_{e_{\psi}} b_{2}\right) \bar{E}\left\{\zeta_{u, 2}(k) \sin (\psi(k-1))\right\} \\
& -\left(-2 \mathcal{X}_{|u| u} \bar{v}_{c, N S}-2 \mathcal{W}_{|u| u} \bar{v}_{w, N S}-\rho_{e_{\psi}} b_{3}\right) \bar{E}\left\{\zeta_{u, 2}(k) \cos (\psi(k-1)) u(k-1)\right\} \\
& -\left(-2 \mathcal{X}_{|u| u} \bar{v}_{c, E W}-2 \mathcal{W}_{|u| u} \bar{v}_{w, E W}-\rho_{e_{\psi}} b_{4}\right) \bar{E}\left\{\zeta_{u, 2}(k) \sin (\psi(k-1)) u(k-1)\right\} \\
& -\left(\mathcal{X}_{|u| u}\left(\bar{E}\left\{v_{c, N S}(k-1)^{2}\right\}-\bar{E}\left\{v_{c, E W}(k-1)^{2}\right\}\right)+\mathcal{W}_{|u| u}\left(\bar{E}\left\{v_{w, N S}(k-1)^{2}\right\}\right.\right. \\
& \left.\left.-\bar{E}\left\{v_{w, E W}(k-1)^{2}\right\}\right)-\left(2 \sigma_{e_{\psi}}-1\right) b_{5}\right) \bar{E}\left\{\zeta_{u, 2}(k) \cos ^{2}(\psi(k-1))\right\} \\
& -\left(\mathcal{X}_{|u| u} \bar{E}\left\{v_{c, N S}(k-1) v_{c, E W}(k-1)\right\}+\mathcal{W}_{|u| u} \bar{E}\left\{v_{w, N S}(k-1) v_{w, E W}(k-1)\right\}\right. \\
& \left.-\left(2 \sigma_{e_{\psi}}-1\right) b_{6}\right) \bar{E}\left\{\zeta_{u, 2}(k) \sin (2 \psi(k-1))\right\} \text {. }
\end{aligned}
$$


From this it can be seen that the asymptotic parameter estimates in (5.27) again follow. The fact that the same parameters are recovered in this case is natural because all the second-order modulus terms that alter sign in the true system, also alter sign in the predictor model. Therefore, even if the sign of some terms in the IV equation changes, the same parameter vector still makes it fulfilled.

An inherently challenging scenario is encountered if $u_{r}(k)$ and $u_{q}(k)$ are of opposite sign. If for example $u_{r}(k)>0, u_{q}(k)<0$ and $u(k)+e_{u}(k)>0$, which could be the case if the ship is moving forward under heavy tail wind, the ship would experience a decelerating effect from the surrounding water and an accelerating effect from the surrounding air. In this situation, it is difficult to get an estimate of the sought sum $\mathcal{X}_{|u| u}+\mathcal{W}_{|u| u}$ and merely an estimate of the difference $\mathcal{X}_{|u| u}-\mathcal{W}_{|u| u}$ would be obtainable. Without adding more measurements it is therefore necessary to assume that the experiments can be performed with positive excitation of sufficient amplitude to fulfill (5.7), or with negative excitation enough to fulfill the opposite condition (5.32). In theory, there could be other conditional regions where the sought sum could be found. For example, if the ship, the ocean current and the wind all were moving in the same direction but the ship was moving at a lower speed than the other two, it could possibly be the case that $u_{r}(k)<0$, $u_{q}(k)<0$ and $u(k)+e_{u}(k)>0$. Then, the sum $\mathcal{X}_{|u| u}+\mathcal{W}_{|u| u}$ could actually be estimated. However, requiring that experiments fulfill a condition like that is not practically feasible, especially in the later analyzed 3-DOF case.

\subsubsection{Including wind measurements}

In general, for being able to uniquely separate the aerodynamic and hydrodynamic damping effects, either the wind velocity or the velocity of the ocean current needs to be measured. It is possible, but not so common, to have sensors for measuring the relative velocity between the ship and the surrounding water. On the other hand, ships are often equipped with means for measuring wind speed and direction. In this section, a way to include wind measurements in the estimation framework is shown. The wind velocity can however be measured in different ways which leads to various challenges. Sensors that measure wind speed are called anemometers and most common is perhaps to measure the wind's speed and direction using a propeller-based anemometer attached to a weather vane, as described in Fossen [2011]. Another option is to use ultrasonic anemometers. Furthermore, the wind sensors are usually mounted onboard the ship and provide relative measurements but in some cases land-based weather stations are used. 


\section{Cartesian measurements in the $b$-frame}

Most convenient from a system identification point of view is to directly have Cartesian measurements in the ship's center of rotation

$$
\begin{aligned}
& y_{u_{q}}(k)=u_{q}(k)+e_{u_{q}}(k), \\
& y_{v_{q}}(k)=v_{q}(k)+e_{v_{q}}(k) .
\end{aligned}
$$

An ultrasonic anemometer measures the time taken for an acoustic pulse to travel from one transducer to another and compares it with the time it takes for a pulse to travel in the reverse direction. The wind speed along the axis spanned by the two transducers can then be calculated from the difference in time of flight. Using four transducers, this can be done both in $x_{b}$ - and $y_{b}$-directions. Cartesian measurements of relative wind in the $b$-frame, like (5.35), are therefore naturally obtained when using ultrasonic anemometers mounted onboard the ship. Notably, if the sensors are not mounted with the ship's center of rotation in mind, the measured relative wind speeds will also depend on the ship's yaw rate. Cartesian measurements in the $b$-frame can also be obtained by mounting two conventional propeller-based anemometers in tunnels aligning with the $x_{b}$ - and $y_{b}$-axis, respectively.

\section{Polar measurements in the $b$-frame}

Use of weather vanes is common but poses some challenges for system identification. There are many ways to represent the wind conditions and one is as earlier, the decomposition into a north/south and an east/west component in the $n$-frame. For discussions about weather-vane measurements, a representation on polar form is however more useful. Therefore, let

$$
V_{q}(k)=\sqrt{u_{q}(k)^{2}+v_{q}(k)^{2}},
$$

denote the relative speed between the ship and the wind and let $\beta_{q}(k)$ denote the relative angle, such that the connection to the decomposed form is

$$
\left[\begin{array}{c}
v_{q, N S}(k) \\
v_{q, E W}(k)
\end{array}\right]=\left[\begin{array}{c}
V_{q}(k) \cos \left(\beta_{q}(k)\right) \\
V_{q}(k) \sin \left(\beta_{q}(k)\right)
\end{array}\right] .
$$

In the $b$-frame, the relative velocities depend both on $\beta_{q}(k)$ and on $\psi(k)$

$$
\left[\begin{array}{l}
u_{q}(k) \\
v_{q}(k)
\end{array}\right]=\left[\begin{array}{c}
V_{q}(k) \cos \left(\beta_{q}(k)-\psi(k)\right) \\
V_{q}(k) \sin \left(\beta_{q}(k)-\psi(k)\right)
\end{array}\right] .
$$

If the angle of attack is defined as in Figure 5.1, then

$$
\gamma_{q}(k)=2 \pi-\beta_{q}(k)+\psi(k)
$$




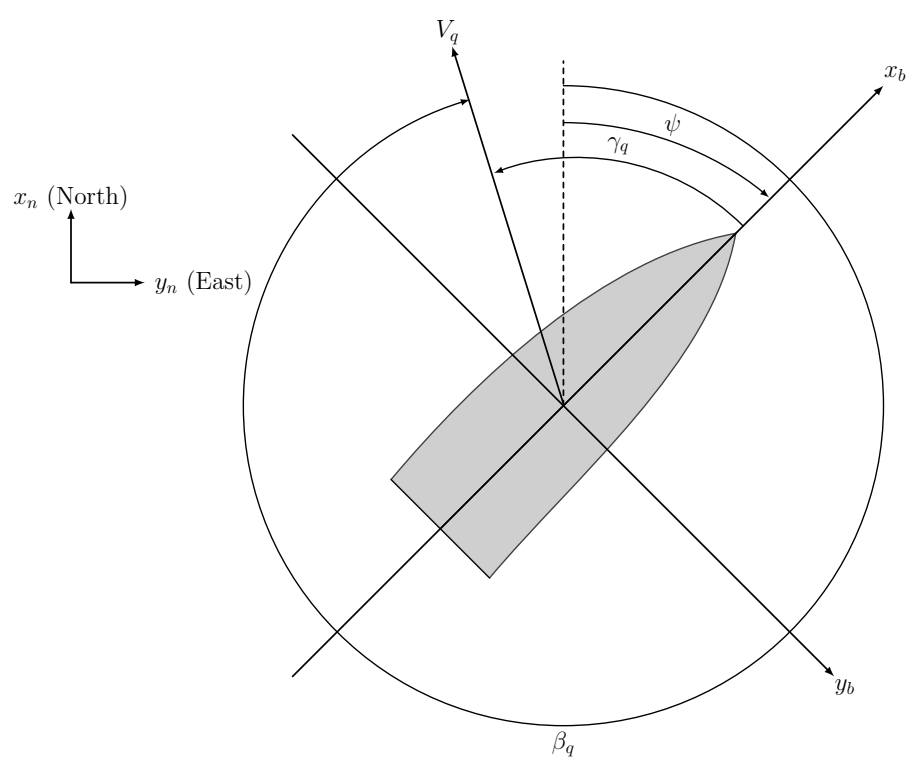

Figure 5.1: Relative speed between the ship and the wind $V_{q}$, relative wind angle $\beta_{q}$ and angle of attack $\gamma_{q}$ relative to the bow of the ship.

and the $b$-frame velocities can equivalently be expressed as

$$
\left[\begin{array}{c}
u_{q}(k) \\
v_{q}(k)
\end{array}\right]=\left[\begin{array}{c}
V_{q}(k) \cos \left(-\gamma_{q}(k)\right) \\
V_{q}(k) \sin \left(-\gamma_{q}(k)\right)
\end{array}\right]=\left[\begin{array}{c}
V_{q}(k) \cos \left(\gamma_{q}(k)\right) \\
-V_{q}(k) \sin \left(\gamma_{q}(k)\right)
\end{array}\right] .
$$

An anemometer mounted together with a weather vane measures the relative speed between the ship and the wind

$$
y_{V_{q}}(k)=V_{q}(k)+e_{V_{q}}(k),
$$

whereas the weather vane itself measures the angle between a reference line on the ship and the vector representing the relative motion between the body and the surrounding air. If the weather vane is mounted in the ship's center of rotation and such that this reference line coincides with the $x_{b}$-axis, it will measure the angle of attack

$$
y_{\gamma_{q}}(k)=\gamma_{q}(k)+e_{\gamma_{q}}(k) .
$$

Using these actual measurements, artificial ones of the corresponding surge and sway components can be formed

$$
\begin{aligned}
& y_{u_{q}}^{\prime}(k)=y_{V_{q}}(k) \cos \left(y_{\gamma_{q}}(k)\right), \\
& y_{v_{q}}^{\prime}(k)=-y_{V_{q}}(k) \sin \left(y_{\gamma_{q}}(k)\right) .
\end{aligned}
$$


Notably, these artificial measurements are not unbiased even if $e_{V_{q}}(k)$ and $e_{\gamma_{q}}(k)$ follow zero-symmetric distributions

$$
\begin{aligned}
\bar{E}\left\{y_{u_{q}}^{\prime}(k)\right\} & =\bar{E}\left\{\left(V_{q}(k)+e_{V_{q}}(k)\right) \cos \left(\gamma_{q}(k)+e_{\gamma_{q}}(k)\right)\right\} \\
& =\bar{E}\left\{V_{q}(k)\left(\cos \left(\gamma_{q}(k)\right) \cos \left(e_{\gamma_{q}}(k)\right)-\sin \left(\gamma_{q}(k)\right) \sin \left(e_{\gamma_{q}}(k)\right)\right)\right\} \\
& =\rho_{e_{\gamma}} V_{q}(k) \cos \left(\gamma_{q}(k)\right)=\rho_{e_{\gamma}} u_{q}(k), \\
\bar{E}\left\{y_{v_{q}}^{\prime}(k)\right\} & =-\bar{E}\left\{\left(V_{q}(k)+e_{V_{q}}(k)\right) \sin \left(\gamma_{q}(k)+e_{\gamma_{q}}(k)\right)\right\} \\
& =-\bar{E}\left\{V_{q}(k)\left(\sin \left(\gamma_{q}(k)\right) \cos \left(e_{\gamma_{q}}(k)\right)+\cos \left(\gamma_{q}(k)\right) \sin \left(e_{\gamma_{q}}(k)\right)\right)\right\} \\
& =-\rho_{e_{\gamma}} V_{q}(k) \sin \left(\gamma_{q}(k)\right)=\rho_{e_{\gamma}} v_{q}(k) .
\end{aligned}
$$

Here, the bias depends on the expectation

$$
\rho_{e_{\gamma}} \triangleq \bar{E}\left\{\cos \left(e_{\gamma_{q}}(k)\right)\right\} \text {. }
$$

If the regressor $y_{u_{q}}^{\prime}(k-1)\left|y_{u_{q}}^{\prime}(k-1)\right|$ is included in the predictor it is consequently not possible to obtain a consistent estimator in the same way as discussed earlier.

\section{Measurements in the $n$-frame}

If the used sensors are land-based, the wind velocity will instead be measured in the $n$-frame and not naturally be relative to the velocity of the ship. In this case, a mapping to the $b$-frame must be carried out before the measurements are used to form regressors. Since the rotation matrix is based on measurements of the yaw angle and not known exactly, this will again cause problems in the same way as discussed in Section 3.6. Provided that the yaw angle is measured with high accuracy, the measurements will still be useful after the coordinate transformation. Then, estimates of the relative velocity can in turn be obtained by use of the already available measurements of the ship's absolute velocity. This is in agreement with Remark 4.6.

\section{Including a wind regressor}

Assume that measurements of relative wind speed in surge direction, (5.35a), are available and that the corresponding measurement disturbances have zero mean and are bounded in magnitude

$$
-\eta_{e_{u_{q}}}<e_{u_{q}}(k)<\eta_{e_{u_{q}}} .
$$


Further, augment the regression vector as

$$
\begin{aligned}
& \varphi_{u, 3}(k)=\left[y_{u}(k-1) \quad y_{u}(k-1)\left|y_{u}(k-1)\right| \quad y_{u_{q}}(k-1)\left|y_{u_{q}}(k-1)\right| \quad \tilde{\tau}_{x}(k-1)\right. \\
& \cos \left(y_{\psi}(k-1)\right) \sin \left(y_{\psi}(k-1)\right)\left|y_{u}(k-1)\right| \cos \left(y_{\psi}(k-1)\right) \\
& \left|y_{u}(k-1)\right| \sin \left(y_{\psi}(k-1)\right) \operatorname{sgn}\left(\left(y_{u}(k-1)\right) \cos ^{2}\left(y_{\psi}(k-1)\right)\right. \\
& \operatorname{sgn}\left(\left(y_{u}(k-1)\right) \sin \left(2 y_{\psi}(k-1)\right)\right]^{T} \text {, }
\end{aligned}
$$

and form the corresponding predictor

$$
\hat{y}_{u, 3}(k \mid \theta)=\varphi_{u, 3}^{T}(k) \cdot\left[\begin{array}{llllll}
\theta_{1} & \ldots & \theta_{4} & b_{1} & \ldots & b_{6}
\end{array}\right]^{T} \triangleq \varphi_{u, 3}^{T}(k) \theta
$$

Now consider the modified experiment condition

$$
\left\{\begin{array}{l}
u(k)>\max \left(\eta_{e_{u}}, \bar{v}_{c, N S}+\eta_{\tilde{v}_{c}}, \bar{v}_{c, E W}+\eta_{\tilde{v}_{c}}\right), \\
u_{q}(k)>\eta_{e_{u_{q}}},
\end{array}\right.
$$

under which (5.6a) can be expressed as

$$
\begin{aligned}
u(k+1) & =u(k)+\mathcal{X}_{u}\left(u(k)-u_{c}(k)\right)+\mathcal{X}_{|u| u}\left(u(k)-u_{c}(k)\right)^{2}+\mathcal{W}_{|u| u} u_{q}(k)^{2} \\
& +\mathcal{X}_{\mu} \tilde{\tau}_{x}(k)=\left(1+\mathcal{X}_{u}-2 \mathcal{X}_{|u| u} u_{c}(k)\right) u(k)+\mathcal{X}_{|u| u} u(k)^{2} \\
& +\mathcal{W}_{|u| u} u_{q}(k)^{2}+\mathcal{X}_{\mu} \tilde{\tau}_{x}(k)-\mathcal{X}_{u} u_{c}(k)+\mathcal{X}_{|u| u} u_{c}(k)^{2}
\end{aligned}
$$

Assume that there exists an instrument vector $\zeta_{u, 3}(k) \in \mathbb{R}^{10}$, which is independent of $e_{u}(k), e_{\psi}(k)$ and $e_{u_{q}}(k)$, has zero mean and fulfills (2.19a). The left-hand side of the IV equation can in this case be expressed as

$$
\begin{aligned}
& \bar{E}\left\{\zeta_{u, 3}(k)\left(y_{u}(k)-\varphi_{u, 3}^{T}(k) \theta\right)\right\}=\bar{E}\left\{\zeta _ { u , 3 } ( k ) \left[\left(1+\mathcal{X}_{u}-2 \mathcal{X}_{|u| u}\left(v_{c, N S}(k-1)\right.\right.\right.\right. \\
& \left.\left.\cdot \cos (\psi(k-1))+v_{c, E W}(k-1) \sin (\psi(k-1))\right)\right) u(k-1)+\mathcal{X}_{|u| u} u(k-1)^{2} \\
& +\mathcal{W}_{|u| u} u_{q}(k-1)^{2}+\mathcal{X}_{\mu} \tilde{\tau}_{x}(k-1)-\mathcal{X}_{u}\left(v_{c, N S}(k-1) \cos (\psi(k-1))\right. \\
& \left.+v_{c, E W}(k-1) \sin (\psi(k-1))\right)+\mathcal{X}_{|u| u}\left(v_{c, N S}(k)^{2} \cos ^{2}(\psi(k-1))\right. \\
& +2 v_{c, N S}(k-1) v_{c, E W}(k-1) \cos (\psi(k-1)) \sin (\psi(k-1)) \\
& \left.+v_{c, E W}(k-1)^{2} \sin ^{2}(\psi(k-1))\right)+e_{u}(k)-\theta_{1}\left(u(k-1)+e_{u}(k-1)\right) \\
& -\theta_{2}\left(u(k-1)^{2}+2 u(k-1) e_{u}(k-1)+e_{u}(k-1)^{2}\right) \\
& -\theta_{3}\left(u_{q}(k-1)^{2}+2 e_{u_{q}}(k-1) u_{q}(k-1)+e_{u_{q}}(k-1)^{2}\right)-\theta_{4} \tilde{\tau}_{x}(k-1)
\end{aligned}
$$




$$
\begin{aligned}
& -b_{1} \cos \left(\psi(k-1)+e_{\psi}(k-1)\right)-b_{2} \sin \left(\psi(k-1)+e_{\psi}(k-1)\right) \\
& -b_{3}\left(u(k-1)+e_{u}(k-1)\right) \cos \left(\psi(k-1)+e_{\psi}(k-1)\right) \\
& -b_{4}\left(u(k-1)+e_{u}(k-1)\right) \sin \left(\psi(k-1)+e_{\psi}(k-1)\right)-b_{5} \cos ^{2}\left(\psi(k-1)+e_{\psi}(k-1)\right) \\
& \left.\left.-b_{6} \sin \left(2\left(\psi(k-1)+e_{\psi}(k-1)\right)\right)\right]\right\} \\
& =\left(1+\mathcal{X}_{u}-\theta_{1}\right) \bar{E}\left\{\zeta_{u, 3}(k) u(k-1)\right\}+\left(\mathcal{X}_{|u| u}-\theta_{2}\right) \bar{E}\left\{\zeta_{u, 3}(k) u(k-1)^{2}\right\} \\
& +\left(\mathcal{W}_{|u| u}-\theta_{3}\right) \bar{E}\left\{\zeta_{u, 3}(k) u_{q}(k-1)^{2}\right\}+\left(\mathcal{X}_{\mu}-\theta_{4}\right) \bar{E}\left\{\zeta_{u, 3}(k) \tilde{\tau}_{x}(k-1)\right\} \\
& +\left(-\mathcal{X}_{u} \bar{v}_{c, N S}-\rho_{e_{\psi}} b_{1}\right) \bar{E}\left\{\zeta_{u, 3}(k) \cos (\psi(k-1))\right\} \\
& +\left(-\mathcal{X}_{u} \bar{v}_{c, E W}-\rho_{e_{\psi}} b_{2}\right) \bar{E}\left\{\zeta_{u, 3}(k) \sin (\psi(k-1))\right\} \\
& +\left(-2 \mathcal{X}_{|u| u} \bar{v}_{c, N S}-\rho_{e_{\psi}} b_{3}\right) \bar{E}\left\{\zeta_{u, 3}(k) \cos (\psi(k-1)) u(k-1)\right\} \\
& +\left(-2 \mathcal{X}_{|u| u} \bar{v}_{c, E W}-\rho_{e_{\psi}} b_{4}\right) \bar{E}\left\{\zeta_{u, 3}(k) \sin (\psi(k-1)) u(k-1)\right\} \\
& +\left(\mathcal{X}_{|u| u}\left(\bar{E}\left\{v_{c, N S}(k-1)^{2}-v_{c, E W}(k-1)^{2}\right\}\right)-\left(2 \sigma_{e_{\psi}}-1\right) b_{5}\right) \bar{E}\left\{\zeta_{u, 3}(k) \cos ^{2}(\psi(k-1))\right\} \\
& +\left(\mathcal{X}_{|u| u} \bar{E}\left\{v_{c, N S}(k-1) v_{c, E W}(k-1)\right\}-\left(2 \sigma_{e_{\psi}}-1\right) b_{6}\right) \bar{E}\left\{\zeta_{u, 3}(k) \sin (2 \psi(k-1))\right\} .
\end{aligned}
$$

From this it can be seen that the asymptotic parameter estimates are

$$
\lim _{N \rightarrow \infty} \hat{\theta}_{N}^{I V}=\left[\begin{array}{c}
1+\mathcal{X}_{u} \\
\mathcal{X}_{|u| u} \\
\mathcal{W}_{|u| u} \\
\mathcal{X}_{\mu} \\
-\frac{1}{\rho_{e_{\psi}}} \mathcal{X}_{u} \bar{v}_{c, N S} \\
-\frac{1}{\rho_{e_{\psi}}} \mathcal{X}_{u} \bar{v}_{c, E W} \\
-\frac{2}{\rho_{e_{\psi}}} \mathcal{X}_{|u| u} \bar{v}_{c, N S} \\
-\frac{2}{\rho_{e_{\psi}}} \mathcal{X}_{|u| u} \bar{v}_{c, E W} \\
\frac{1}{2 \sigma_{e_{\psi}}-1} \mathcal{X}_{|u| u}\left(\bar{E}\left\{v_{c, N S}(k-1)^{2}\right\}-\bar{E}\left\{v_{c, E W}(k-1)^{2}\right\}\right) \\
\frac{1}{2 \sigma_{e_{\psi}}-1} \mathcal{X}_{|u| u} \bar{E}\left\{v_{c, N S}(k-1) v_{c, E W}(k-1)\right\}
\end{array}\right]=\left[\begin{array}{c}
\theta_{0}^{*} \\
\sim
\end{array}\right] .
$$

As before, it is possible to instead perform the experiments such that the converse condition

$$
\left\{\begin{array}{l}
u(k)<-\max \left(\eta_{e_{u}}, \bar{v}_{c, N S}+\eta_{\tilde{v}_{c}}, \bar{v}_{c, E W}+\eta_{\tilde{v}_{c}}\right), \\
u_{q}(k)<-\eta_{e_{u_{q}}},
\end{array}\right.
$$


is fulfilled. In this case, the sign of terms coming from modulus functions in expression (5.52) changes but as in the previously analyzed scenario without supplementary wind measurements, the asymptotic parameter estimates are unaffected. Moreover, since the relative velocity between the ship and the surrounding air is measured separately, it is no longer necessary to perform experiments where $u_{q}(k)$ is of the same sign as $u_{r}(k)$. In other words, any of the conditions

$$
\left\{\begin{array}{l}
u(k)<-\max \left(\eta_{e_{u}}, \bar{v}_{c, N S}+\eta_{\tilde{v}_{c}}, \bar{v}_{c, E W}+\eta_{\tilde{v}_{c}}\right), \\
u_{q}(k)>\eta_{e_{u_{q}}},
\end{array}\right.
$$

or

$$
\left\{\begin{array}{l}
u(k)>\max \left(\eta_{e_{u}}, \bar{v}_{c, N S}+\eta_{\tilde{v}_{c}}, \bar{v}_{c, E W}+\eta_{\tilde{v}_{c}}\right), \\
u_{q}(k)<-\eta_{e_{u_{q}}},
\end{array}\right.
$$

can instead be met. This changes the sign of the aerodynamic term, both in the true system equation and in the predictor and does consequently not affect the asymptotic parameter estimates. The fact that (5.55) and (5.56) can be considered is important, because the wind speed is sometimes higher than the maximum speed of the ship which makes conditions like (5.50) and (5.54) hard to meet. This is especially true when models with multiple DOF are considered. Finally and perhaps most importantly, when wind measurements are included in the framework it is possible to uniquely identify the hydrodynamic and aerodynamic damping as separate effects. This added information can be utilized at a later stage when ship motion is predicted.

\subsection{Maneuvering model}

Now consider the maneuvering model (3.51). Assume that data is generated based on that model with unit sampling time and that all the absolute-velocity states as well as the yaw angle and relative wind velocity components are measured according to

$$
\begin{aligned}
{\left[\begin{array}{l}
u(k) \\
v(k) \\
r(k)
\end{array}\right]=} & {\left[\begin{array}{l}
u(k-1) \\
v(k-1) \\
r(k-1)
\end{array}\right]+\left[\begin{array}{ccc}
\varphi_{u, 0}^{T}(k) & 0 & 0 \\
0 & \varphi_{v, 0}^{T}(k) & 0 \\
0 & 0 & \varphi_{r, 0}^{T}(k)
\end{array}\right] \theta_{0}, } \\
\mathbf{y}(k)= & {\left[\begin{array}{l}
y_{u}(k) \\
y_{v}(k) \\
y_{r}(k) \\
y_{\psi}(k) \\
y_{u_{q}}(k) \\
y_{v_{q}}(k)
\end{array}\right]=\left[\begin{array}{c}
u(k) \\
v(k) \\
r(k) \\
\psi(k) \\
u_{q}(k) \\
v_{q}(k)
\end{array}\right]+\left[\begin{array}{c}
e_{u}(k) \\
e_{v}(k) \\
e_{r}(k) \\
e_{\psi}(k) \\
e_{u_{q}}(k) \\
e_{v_{q}}(k)
\end{array}\right] . }
\end{aligned}
$$

Here, the added subscript, 0 , indicates that this is now considered to be the true system. As before, assume that the measurement and environmental distur- 
bances are bounded in magnitude and that the measurement disturbances follow zero-symmetric distributions.

The same trick of augmenting the regression matrix with bias-capturing elements, can be applied also in this case. Subsequently, the asymptotic results of a set of IV estimators will be provided. Due to the diagonal structure of (5.57a) and the fact that one-step-ahead predictors are considered, models for each DOF can be analyzed separately. For each DOF, the analysis covers both the scenario with supplementary wind measurements and the scenario without. The proofs are quite straightforward but require tedious calculations and the interested reader will find additional justification for the claimed results in the appendix of this chapter.

It will frequently be assumed that the experiments fulfill a set of fairly restrictive assumptions like (5.50), (5.54), (5.55) or (5.56). It should be stressed that it is not necessary to focus on fulfilling a complete set of assumptions directly when the experiments are performed. This is the case because as pointed out in Remark 4.2, it is possible to later form new shorter datasets where each one fulfills one set of assumptions.

\subsubsection{The surge equation}

The surge state equation in (3.51) is

$$
\begin{aligned}
u(k+1)= & u(k)+\mathcal{X}_{v r} v_{r}(k) r(k)+\mathcal{X}_{u} u_{r}(k)+\mathcal{X}_{|u| u \mid}\left|u_{r}(k)\right| u_{r}(k) \\
& +\mathcal{W}_{|u| u \mid}\left|u_{q}(k)\right| u_{q}(k)+\mathcal{X}_{\mu} \tilde{\tau}_{x}(k)+\mathcal{X}_{\kappa} u_{r}(k) \tilde{\tau}_{x}(k) .
\end{aligned}
$$

A similar case was covered in the Section 5.1 but in (5.58) there are two additional terms in comparison to (5.6a), proportional to $v_{r}(k) r(k)$ and $u_{r}(k) \tilde{\tau}_{x}(k)$, respectively. Expanding these gives

$$
\begin{aligned}
v_{r}(k) r(k) & =\left(v(k)+v_{c, N S} \sin (\psi(k))-v_{c, E W} \cos (\psi(k))\right) r(k) \\
& =v(k) r(k)+v_{c, N S} r(k) \sin (\psi(k))-v_{c, E W} r(k) \cos (\psi(k)),
\end{aligned}
$$

and

$$
\begin{aligned}
u_{r}(k) \tilde{\tau}_{x}(k) & =\left(u(k)-v_{c, N S} \cos (\psi(k))-v_{c, E W} \sin (\psi(k))\right) \tilde{\tau}_{x}(k) \\
& =u(k) \tilde{\tau}_{x}(k)-v_{c, N S} \tilde{\tau}_{x}(k) \cos (\psi(k))-v_{c, E W} \tilde{\tau}_{x}(k) \sin (\psi(k)),
\end{aligned}
$$

which means that on top of the previous two regressors, four additional regressors need to be added for capturing bias effects. 


\section{No wind measurement}

In the case where the relative wind velocity is not measured, the regression vector

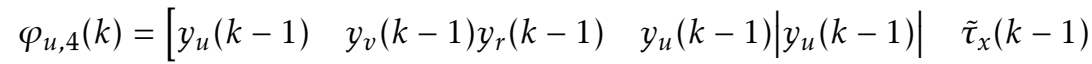

$$
\begin{aligned}
& y_{u}(k-1) \tilde{\tau}_{x}(k-1) \quad \cos \left(y_{\psi}(k-1)\right) \sin \left(y_{\psi}(k-1)\right) \\
& \left|y_{u}(k-1)\right| \cos \left(y_{\psi}(k-1)\right)\left|y_{u}(k-1)\right| \sin \left(y_{\psi}(k-1)\right) \\
& y_{r}(k-1) \cos \left(y_{\psi}(k-1)\right) \quad y_{r}(k-1) \sin \left(y_{\psi}(k-1)\right) \\
& \tilde{\tau}_{x}(k-1) \cos \left(y_{\psi}(k-1)\right) \quad \tilde{\tau}_{x}(k-1) \sin \left(y_{\psi}(k-1)\right) \\
& \operatorname{sgn}\left(\left(y_{u}(k-1)\right) \cos ^{2}\left(y_{\psi}(k-1)\right) \operatorname{sgn}\left(\left(y_{u}(k-1)\right) \sin \left(2 y_{\psi}(k-1)\right)\right]^{T},\right.
\end{aligned}
$$

and its corresponding predictor

$$
\hat{y}_{u, 4}(k \mid \theta)=\varphi_{u, 4}^{T}(k) \cdot\left[\begin{array}{llllll}
\theta_{1} & \ldots & \theta_{5} & b_{1} & \ldots & b_{10}
\end{array}\right]^{T} \triangleq \varphi_{u, 4}^{T}(k) \theta,
$$

can be used. Assume that the experiment design fulfills (5.7) or (5.32). Also assume that there exists an instrument vector $\zeta_{u, 4}(k) \in \mathbb{R}^{15}$, which is independent of $e_{u}(k), e_{v}(k), e_{r}(k)$ and $e_{\psi}(k)$, has zero mean and fulfills (2.19a). Then an IV estimator can be shown to asymptotically give the parameter estimates

$$
\lim _{N \rightarrow \infty} \hat{\theta}_{N}^{I V}=\left[\begin{array}{c}
1+\mathcal{X}_{u} \\
\mathcal{X}_{v r} \\
\mathcal{X}_{|u| u}+\mathcal{W}_{|u| u} \\
\mathcal{X}_{\mu} \\
\mathcal{X}_{\mathcal{K}} \\
-\frac{1}{\rho_{e_{\psi}}} \mathcal{X}_{u} \bar{v}_{c, N S} \\
-\frac{1}{\rho_{e_{\psi}}} \mathcal{X}_{u} \bar{v}_{c, E W} \\
\frac{1}{\rho_{e_{\psi}}}\left(-2 \mathcal{X}_{|u| u} \bar{v}_{c, N S}-2 \mathcal{W}_{|u| u} \bar{v}_{w, N S}\right) \\
\frac{1}{\rho_{e_{\psi}}}\left(-2 \mathcal{X}_{|u| u} \bar{v}_{c, E W}-2 \mathcal{W}_{|u| u} \bar{v}_{w, E W}\right) \\
-\frac{1}{\rho_{e_{\psi}}} \mathcal{X}_{v r} \bar{v}_{c, E W} \\
\frac{1}{\rho_{e_{\psi}}} \mathcal{X}_{v r} \bar{v}_{c, N S} \\
-\frac{1}{\rho_{e_{\psi}}} \mathcal{X}_{\kappa} \bar{v}_{c, N S} \\
-\frac{1}{\rho_{e_{\psi}}} \mathcal{X}_{\kappa} \bar{v}_{c, E W} \\
\frac{1}{2 \sigma_{e_{\psi}}-1} \xi_{1}\left(v_{c, n}, v_{w, n}\right) \\
\frac{1}{2 \sigma_{e_{\psi}}-1} \xi_{2}\left(v_{c, n}, v_{w, n}\right)
\end{array}\right],
$$

where $\xi_{1}($.$) and \xi_{2}($.$) are defined by (5.28) and (5.29), respectively. This is a consis-$ tent estimator of the parameters needed for performing undisturbed simulations 
of (5.58) and in addition the estimator provides information about the first and second-order moments of the environmental disturbances. An explicit expression for the left-hand side of the IV equation is given by (5.92) in the appendix of this chapter.

\section{With wind measurement}

There is no wind disturbance present in the terms $\mathcal{X}_{v r} v_{r}(k) r(k)$ and $\mathcal{X}_{\kappa} u_{r}(k) \tilde{\tau}_{x}(k)$. Therefore, the predictor (5.49) can readily be augmented with the same six regressors as above

$$
\begin{aligned}
& \varphi_{u, 5}(k)=\left[y_{u}(k-1) \quad y_{v}(k-1) y_{r}(k-1) \quad y_{u}(k-1)\left|y_{u}(k-1)\right| \quad y_{u_{q}}(k-1)\left|y_{u_{q}}(k-1)\right|\right. \\
& \tilde{\tau}_{x}(k-1) \quad y_{u}(k-1) \tilde{\tau}_{x}(k-1) \quad \cos \left(y_{\psi}(k-1)\right) \quad \sin \left(y_{\psi}(k-1)\right) \\
& \left|y_{u}(k-1)\right| \cos \left(y_{\psi}(k-1)\right) \quad\left|y_{u}(k-1)\right| \sin \left(y_{\psi}(k-1)\right) \quad y_{r}(k-1) \cos \left(y_{\psi}(k-1)\right) \\
& y_{r}(k-1) \sin \left(y_{\psi}(k-1)\right) \quad \tilde{\tau}_{x}(k-1) \cos \left(y_{\psi}(k-1)\right) \quad \tilde{\tau}_{x}(k-1) \sin \left(y_{\psi}(k-1)\right) \\
& \operatorname{sgn}\left(\left(y_{u}(k-1)\right) \cos ^{2}\left(y_{\psi}(k-1)\right) \operatorname{sgn}\left(\left(y_{u}(k-1)\right) \sin \left(2 y_{\psi}(k-1)\right)\right]^{T}\right. \text {, }
\end{aligned}
$$

$$
\hat{y}_{u, 5}(k \mid \theta)=\varphi_{u, 5}^{T}(k) \cdot\left[\begin{array}{llllll}
\theta_{1} & \ldots & \theta_{6} & b_{1} & \ldots & b_{10}
\end{array}\right]^{T} \triangleq \varphi_{u, 5}^{T}(k) \theta
$$

Assume that the experiments fulfill any of the conditions (5.50), (5.54), (5.55) or (5.56). Also assume that there exists an instrument vector $\zeta_{u, 5}(k) \in \mathbb{R}^{16}$, which is independent of $e_{u}(k), e_{v}(k), e_{r}(k), e_{\psi}(k)$ and $e_{u_{q}}(k)$, has zero mean and fulfills 
(2.19a). Then an IV estimator can be shown to asymptotically give

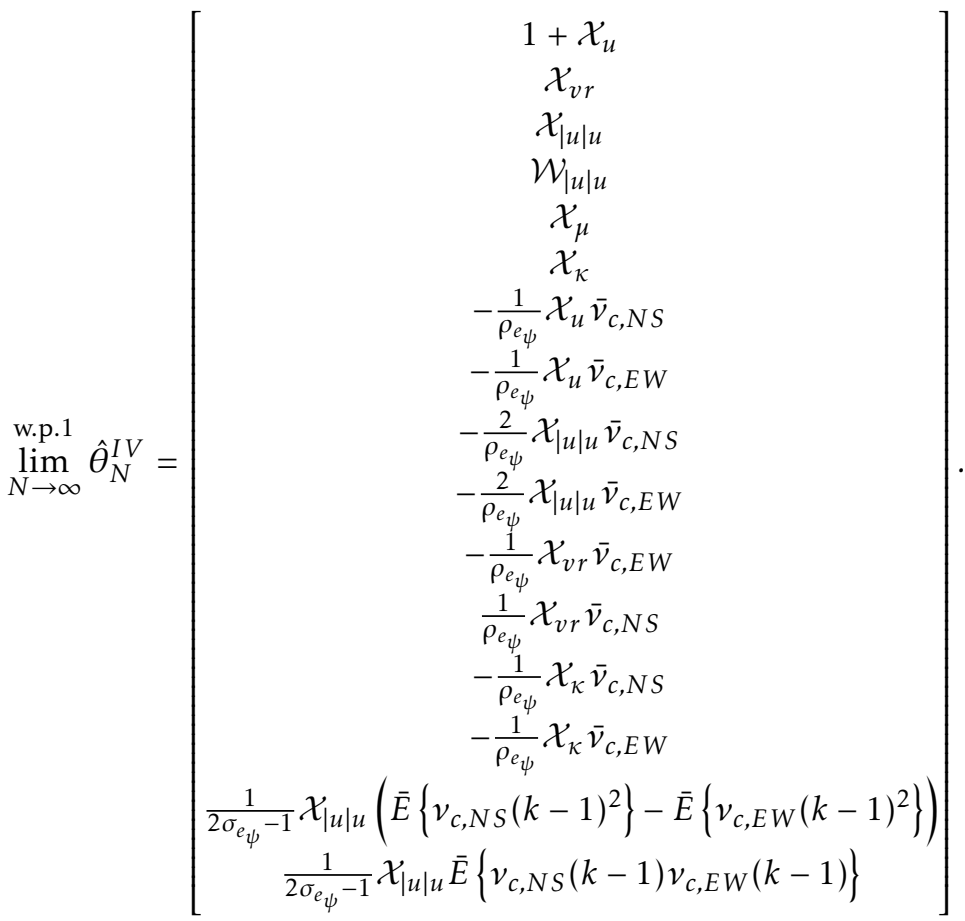

This is also a consistent estimator of the parameters needed for performing undisturbed simulations of (5.58). Moreover, since the relative wind speed $u_{q}(k)$ is measured separately it is possible to uniquely identify the hydrodynamic and aerodynamic damping as separate effects. The asymptotic parameter estimates (5.66) follow from the discussion in Section 5.1.4 together with the reasoning about (5.92), which is given in the appendix of this chapter.

\subsubsection{The sway equation}

The sway state equation in (3.51) is

$$
\begin{aligned}
v(k+1) & =v(k)+\mathcal{Y}_{u r} u_{r}(k) r(k)+\mathcal{Y}_{v} v_{r}(k)+\mathcal{Y}_{|v| v \mid}\left|v_{r}(k)\right| v_{r}(k) \\
& +\mathcal{Y}_{|v| r \mid}\left|v_{r}(k)\right| r(k)+\mathcal{W}_{|v| v \mid}\left|v_{q}(k)\right| v_{q}(k)+\mathcal{Y}_{\mu} \tilde{\tau}_{y}(k) .
\end{aligned}
$$

Notably, only $v_{r}(k)$ and $v_{q}(k)$ appear in the modulus functions.

\section{No wind measurement}

In the case of not measuring the relative wind velocity, assume that the experiments are performed such that $v_{r}(k)>0, v_{q}(k)>0$ and $v(k)+e_{v}(k)>0$ or such 
that $v_{r}(k)<0, v_{q}(k)<0$ and $v(k)+e_{v}(k)<0$. By the previous assumptions $(5.4)$ and (5.5), sufficient conditions for fulfilling this are

$$
\begin{aligned}
& v(k)>\max \left(\eta_{e_{v}}, \bar{v}_{c, N S}+\eta_{\tilde{v}_{c}}, \bar{v}_{c, E W}+\eta_{\tilde{v}_{c}}, \bar{v}_{w, N S}+\eta_{\tilde{v}_{w}}, \bar{v}_{w, E W}+\eta_{\tilde{v}_{w}}\right), \\
& v(k)<-\max \left(\eta_{e_{v}}, \bar{v}_{c, N S}+\eta_{\tilde{v}_{c}}, \bar{v}_{c, E W}+\eta_{\tilde{v}_{c}}, \bar{v}_{w, N S}+\eta_{\tilde{v}_{w}}, \bar{v}_{w, E W}+\eta_{\tilde{v}_{w}}\right),
\end{aligned}
$$

respectively. Consider in this case the regression vector

$$
\begin{aligned}
& \varphi_{v, 1}(k)=\left[y_{v}(k-1) \quad y_{u}(k-1) y_{r}(k-1) \quad y_{v}(k-1)\left|y_{v}(k-1)\right| \quad y_{r}(k-1)\left|y_{v}(k-1)\right|\right. \\
& \tilde{\tau}_{y}(k-1) \cos \left(y_{\psi}(k-1)\right) \sin \left(y_{\psi}(k-1)\right)\left|y_{v}(k-1)\right| \cos \left(y_{\psi}(k-1)\right) \\
& \left|y_{v}(k-1)\right| \sin \left(y_{\psi}(k-1)\right) \quad y_{r}(k-1) \cos \left(y_{\psi}(k-1)\right) \\
& y_{r}(k-1) \sin \left(y_{\psi}(k-1)\right) \operatorname{sgn}\left(\left(y_{v}(k-1)\right) \cos ^{2}\left(y_{\psi}(k-1)\right)\right. \\
& \operatorname{sgn}\left(\left(y_{v}(k-1)\right) \sin \left(2 y_{\psi}(k-1)\right)\right]^{T} \text {, }
\end{aligned}
$$

and the corresponding sway predictor

$$
\hat{y}_{v, 1}(k \mid \theta)=\varphi_{v, 1}^{T}(k) \cdot\left[\begin{array}{llllll}
\theta_{1} & \ldots & \theta_{5} & b_{1} & \ldots & b_{8}
\end{array}\right]^{T} \triangleq \varphi_{v, 1}^{T}(k) \theta .
$$

Assume that there exists an instrument vector $\zeta_{v, 1}(k) \in \mathbb{R}^{13}$, which is independent of $e_{u}(k), e_{v}(k), e_{r}(k)$ and $e_{\psi}(k)$, has zero mean and fulfills (2.19a). Then an IV estimator can be shown to asymptotically give the parameter estimates

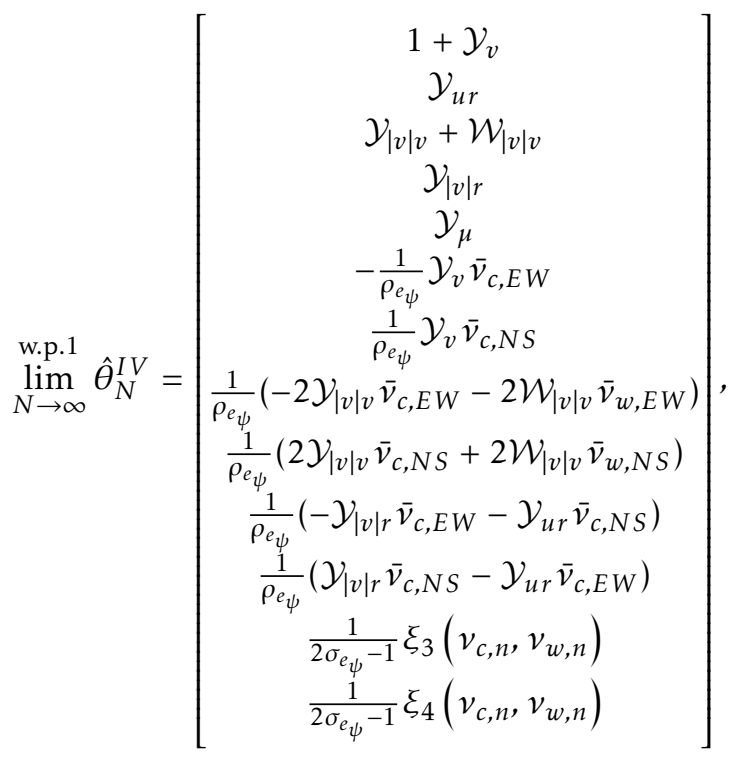


where

$$
\begin{aligned}
\xi_{3}\left(v_{c, n}, v_{w, n}\right)=\mathcal{Y}_{|v| v}\left(\bar{E}\left\{v_{c, E W}(k-1)^{2}\right\}-\bar{E}\left\{v_{c, N S}(k-1)^{2}\right\}\right) & \\
& +\mathcal{W}_{|v| v}\left(\bar{E}\left\{v_{w, E W}(k-1)^{2}\right\}-\bar{E}\left\{v_{w, N S}(k-1)^{2}\right\}\right), \\
\xi_{4}\left(v_{c, n}, v_{w, n}\right)=-\mathcal{Y}_{|v| v} \bar{E}\{ & \left.v_{c, N S}(k-1) v_{c, E W}(k-1)\right\} \\
& -\mathcal{W}_{|v| v} \bar{E}\left\{v_{w, N S}(k-1) v_{w, E W}(k-1)\right\} .
\end{aligned}
$$

The proof is provided in the appendix of this chapter. This is a consistent estimator of the parameters needed for performing undisturbed simulations of (5.67) and in addition the estimator provides information about the first and secondorder moments of the environmental disturbances.

\section{With wind measurement}

Similarly to the surge case, wind measurements can readily be included in the predictor

$$
\begin{aligned}
& \varphi_{v, 2}(k)=\left[y_{v}(k-1) \quad y_{u}(k-1) y_{r}(k-1) \quad y_{v}(k-1)\left|y_{v}(k-1)\right| \quad y_{r}(k-1)\left|y_{v}(k-1)\right|\right. \\
& y_{v_{q}}^{\prime}(k-1)\left|y_{v_{q}}^{\prime}(k-1)\right| \quad \tilde{\tau}_{y}(k-1) \quad \cos \left(y_{\psi}(k-1)\right) \quad \sin \left(y_{\psi}(k-1)\right) \\
& \left|y_{v}(k-1)\right| \cos \left(y_{\psi}(k-1)\right) \quad\left|y_{v}(k-1)\right| \sin \left(y_{\psi}(k-1)\right) \quad y_{r}(k-1) \cos \left(y_{\psi}(k-1)\right) \\
& y_{r}(k-1) \sin \left(y_{\psi}(k-1)\right) \operatorname{sgn}\left(\left(y_{v}(k-1)\right) \cos ^{2}\left(y_{\psi}(k-1)\right)\right. \\
& \operatorname{sgn}\left(\left(y_{v}(k-1)\right) \sin \left(2 y_{\psi}(k-1)\right)\right]^{T} \text {, } \\
& \hat{y}_{v, 2}(k \mid \theta)=\varphi_{v, 2}^{T}(k) \cdot\left[\begin{array}{llllll}
\theta_{1} & \ldots & \theta_{6} & b_{1} & \ldots & b_{8}
\end{array}\right]^{T} \triangleq \varphi_{v, 2}^{T}(k) \theta
\end{aligned}
$$

In this case, assume that any of the conditions

$$
\begin{aligned}
& \left\{\begin{array}{l}
v(k)>\max \left(\eta_{e_{v}}, \bar{v}_{c, N S}+\eta_{\tilde{v}_{c}}, \bar{v}_{c, E W}+\eta_{\tilde{v}_{c}}\right), \\
v_{q}(k)>\eta_{e_{v_{q}}},
\end{array}\right. \\
& \left\{\begin{array}{l}
v(k)<-\max \left(\eta_{e_{v}}, \bar{v}_{c, N S}+\eta_{\tilde{v}_{c}}, \bar{v}_{c, E W}+\eta_{\tilde{v}_{c}}\right), \\
v_{q}(k)>\eta_{e_{v_{q}}},
\end{array}\right. \\
& \left\{\begin{array}{l}
v(k)>\max \left(\eta_{e_{v}}, \bar{v}_{c, N S}+\eta_{\tilde{v}_{c}}, \bar{v}_{c, E W}+\eta_{\tilde{v}_{c}}\right), \\
v_{q}(k)<-\eta_{e_{v_{q}}},
\end{array}\right.
\end{aligned}
$$




$$
\left\{\begin{array}{l}
v(k)<-\max \left(\eta_{e_{v}}, \bar{v}_{c, N S}+\eta_{\tilde{v}_{c}}, \bar{v}_{c, E W}+\eta_{\tilde{v}_{c}}\right), \\
v_{q}(k)<-\eta_{e_{v_{q}}}
\end{array}\right.
$$

are met. Provided that there exists an instrument vector $\zeta_{v, 2}(k) \in \mathbb{R}^{14}$, which is independent of $e_{u}(k), e_{v}(k), e_{r}(k), e_{\psi}(k)$ and $e_{v_{q}}(k)$, has zero mean and fulfills (2.19a), an IV estimator can be shown to asymptotically give

$$
\lim _{N \rightarrow \infty} \hat{\theta}_{N}^{I V}=\left[\begin{array}{c}
1+\mathcal{Y}_{v} \\
\mathcal{Y}_{u r} \\
\mathcal{Y}_{|v| v} \\
\mathcal{Y}_{|v| r} \\
\mathcal{W}_{|v| v} \\
\mathcal{Y}_{\mu} \\
-\frac{1}{\rho_{e_{\psi}}} \mathcal{Y}_{v} \bar{v}_{c, E W} \\
\frac{1}{\rho_{e_{\psi}}} \mathcal{Y}_{v} \bar{v}_{c, N S} \\
-\frac{2}{\rho_{e_{\psi}}} \mathcal{Y}_{|v| v} \bar{v}_{c, E W} \\
\frac{2}{\rho_{e_{\psi}}} \mathcal{Y}_{|v| v} \bar{v}_{c, N S} \\
\frac{1}{\rho_{e_{\psi}}}\left(-\mathcal{Y}_{|v| r} \bar{\nu}_{c, E W}-\mathcal{Y}_{u r} \bar{\nu}_{c, N S}\right) \\
\frac{1}{\rho_{e_{\psi}}}\left(\mathcal{Y}_{|v| r} \bar{\nu}_{c, N S}-\mathcal{Y}_{u r} \bar{v}_{c, E W}\right) \\
\frac{1}{2 \sigma_{e_{\psi}}-1} \mathcal{Y}_{|v| v}\left(\bar{E}\left\{v_{c, E W}(k-1)^{2}-v_{c, N S}(k-1)^{2}\right\}\right) \\
\frac{1}{2 \sigma_{e_{\psi}}-1} \mathcal{Y}_{|v| v} \bar{E}\left\{v_{c, N S}(k-1) v_{c, E W}(k-1)\right\}
\end{array}\right] .
$$

The proof is provided in the appendix of this chapter. This is also a consistent estimator of the parameters needed for performing undisturbed simulations of (5.67). Moreover, since the relative wind speed $v_{q}(k)$ is measured separately it is possible to uniquely identify the hydrodynamic and aerodynamic damping as separate effects.

\subsubsection{The yaw-rate equation}

The yaw-rate equation in (3.51) is

$$
\begin{aligned}
r(k+1) & =r(k)+\mathcal{N}_{u v} u_{r}(k) v_{r}(k)+\mathcal{N}_{v} r(k)+\mathcal{N}_{|v| v}\left|v_{r}(k)\right| v_{r}(k) \\
& +\mathcal{N}_{|v| r \mid}\left|v_{r}(k)\right| r(k)+\mathcal{W}_{u v} u_{q}(k) v_{q}(k)+\mathcal{N}_{\mu} \tilde{\tau}_{\psi}(k) .
\end{aligned}
$$

Finding a general predictor that gives the same estimates of the disturbance parameters for positive and negative sway velocities is hard for this state equation. This is because bias-capturing regressors on the forms $y_{v}(k-1) \cos \left(y_{\psi}(k-1)\right)$ and $y_{v}(k-1) \sin \left(y_{\psi}(k-1)\right)$ are needed to capture bias terms that come from terms that both do and do not include modulus functions. In this case, a predictor that 
gives accurate estimates of the parameters needed for performing undisturbed simulations of the system, regardless of excitation direction, will therefore be deemed sufficient.

\section{No wind measurement}

Assume that the experiments are performed such that any of the conditions

$$
\begin{aligned}
& v(k)>\max \left(\eta_{e_{v}}, \bar{v}_{c, N S}+\eta_{\tilde{v}_{c}}, \bar{v}_{c, E W}+\eta_{\tilde{v}_{c}}\right), \\
& v(k)<-\max \left(\eta_{e_{v}}, \bar{v}_{c, N S}+\eta_{\tilde{v}_{c}}, \bar{v}_{c, E W}+\eta_{\tilde{v}_{c}}\right),
\end{aligned}
$$

are met. Notably, fulfilling any of the experiment conditions used for the sway equation, (5.68), (5.69), (5.77), (5.78), (5.79) or (5.80), implies fulfillment of (5.83) or (5.84) as well. The reason that milder experiment conditions can be considered here is that no term including the absolute value of a relative wind-velocity component is present in (5.82). Further, now consider the regression vector

$$
\begin{aligned}
& \varphi_{r, 1}(k)=\left[y_{r}(k-1) \quad y_{u}(k-1) y_{v}(k-1) \quad y_{v}(k-1)\left|y_{v}(k-1)\right| \quad y_{r}(k-1)\left|y_{v}(k-1)\right|\right. \\
& \tilde{\tau}_{\psi}(k-1) \quad y_{r}(k-1) \cos \left(y_{\psi}(k-1)\right) \quad y_{r}(k-1) \sin \left(y_{\psi}(k-1)\right) \\
& y_{v}(k-1) \cos \left(y_{\psi}(k-1)\right) \quad y_{v}(k-1) \sin \left(y_{\psi}(k-1)\right) \\
& y_{u}(k-1) \cos \left(y_{\psi}(k-1)\right) \quad y_{u}(k-1) \sin \left(y_{\psi}(k-1)\right) \\
& \left.\cos ^{2}\left(y_{\psi}(k-1)\right) \sin \left(2 y_{\psi}(k-1)\right)\right]^{T} \text {, }
\end{aligned}
$$

and the corresponding predictor

$$
\hat{y}_{r, 1}(k \mid \theta)=\varphi_{r, 1}^{T}(k) \cdot\left[\begin{array}{llllll}
\theta_{1} & \ldots & \theta_{5} & b_{1} & \ldots & b_{8}
\end{array}\right]^{T} \triangleq \varphi_{r, 1}^{T}(k) \theta .
$$

Also assume that there exists an instrument vector $\zeta_{r, 1}(k) \in \mathbb{R}^{13}$, which is independent of $e_{u}(k), e_{v}(k), e_{r}(k)$ and $e_{\psi}(k)$, has zero mean and fulfills (2.19a). In this case, an IV estimator can be shown to asymptotically give

$$
\lim _{N \rightarrow \infty} \hat{\theta}_{N}^{I V}=\left[\begin{array}{c}
1+\mathcal{N}_{r} \\
\mathcal{N}_{u v}+\mathcal{W}_{u v} \\
\mathcal{N}_{|v| v} \\
\mathcal{N}_{|v| r} \\
\mathcal{N}_{\mu} \\
\sim
\end{array}\right] \text {. }
$$

The proof is provided in the appendix of this chapter. This is a consistent estimator of the parameters needed for performing undisturbed simulations of (5.82). 


\section{With wind measurement}

Wind measurements can readily be included in a yaw-rate predictor as well, such that

$$
\begin{aligned}
& \varphi_{r, 2}(k)=\left[y_{r}(k-1) \quad y_{u}(k-1) y_{v}(k-1) \quad y_{v}(k-1)\left|y_{v}(k-1)\right| \quad y_{r}(k-1)\left|y_{v}(k-1)\right|\right. \\
& y_{u_{q}}(k-1) y_{v_{q}}(k-1) \quad \tilde{\tau}_{\psi}(k-1) \quad y_{r}(k-1) \cos \left(y_{\psi}(k-1)\right) \\
& y_{r}(k-1) \sin \left(y_{\psi}(k-1)\right) \quad y_{v}(k-1) \cos \left(y_{\psi}(k-1)\right) \\
& y_{v}(k-1) \sin \left(y_{\psi}(k-1)\right) \quad y_{u}(k-1) \cos \left(y_{\psi}(k-1)\right) \\
& \left.y_{u}(k-1) \sin \left(y_{\psi}(k-1)\right) \quad \cos ^{2}\left(y_{\psi}(k-1)\right) \sin \left(2 y_{\psi}(k-1)\right)\right]^{T} \text {, } \\
& \hat{y}_{r, 2}(k \mid \theta)=\varphi_{r, 2}^{T}(k) \cdot\left[\begin{array}{llllll}
\theta_{1} & \ldots & \theta_{6} & b_{1} & \ldots & b_{8}
\end{array}\right]^{T} \triangleq \varphi_{r, 2}^{T}(k) \theta .
\end{aligned}
$$

Since no term that includes the absolute value of a relative wind-velocity component is present in (5.82), the same experiment conditions, (5.83) and (5.84), can be considered in this case as well. As earlier, assume that there exists an instrument vector $\zeta_{r, 2}(k) \in \mathbb{R}^{13}$, which is independent of $e_{u}(k), e_{v}(k), e_{r}(k), e_{\psi}(k), e_{u_{q}}(k)$ and $e_{v_{q}}(k)$, has zero mean and fulfills (2.19a). Then an IV estimator can be shown to asymptotically give

$$
\lim _{N \rightarrow \infty} \hat{\theta}_{N}^{I V}=\left[\begin{array}{c}
1+\mathcal{N}_{r} \\
\mathcal{N}_{u v} \\
\mathcal{N}_{|v| v} \\
\mathcal{N}_{|v| r} \\
\mathcal{W}_{u v} \\
\mathcal{N}_{\mu} \\
\sim
\end{array}\right] .
$$

The proof is provided in the appendix of this chapter. This is also a consistent estimator of the parameters needed for performing undisturbed simulations of (5.82). Moreover, since the relative wind velocity components, $u_{q}(k)$ and $v_{q}(k)$, are measured separately it is possible to uniquely identify the hydrodynamic and aerodynamic damping as separate effects.

In summary, consistent estimators of the parameters needed for performing undisturbed simulations of the maneuvering model (3.51) have been suggested. Both for the scenario with supplementary wind measurements and for the scenario without. 


\section{Appendix}

\section{A Asymptotic model residuals}

In this appendix, justification is provided for results proclaimed in Section 5.2.

\section{A.1 Surge equation - without wind measurements}

Under assumption of an experiment design fulfilling (5.7), (5.58) may be rewritten as

$$
\begin{aligned}
u(k+1) & =u(k)+\mathcal{X}_{v r}\left(v(k)-v_{c}(k)\right) r(k)+\mathcal{X}_{u}\left(u(k)-u_{c}(k)\right)+\mathcal{X}_{|u| u}\left(u(k)-u_{c}(k)\right)^{2} \\
& +\mathcal{W}_{|u| u}\left(u(k)-u_{w}(k)\right)^{2}+\mathcal{X}_{\mu} \tilde{\tau}_{x}(k)+\mathcal{X}_{\kappa}\left(u(k)-u_{c}(k)\right) \tilde{\tau}_{x}(k) \\
& =\left(1+\mathcal{X}_{u}-2 \mathcal{X}_{|u| u} u_{c}(k)-2 \mathcal{W}_{|u| u} u_{w}(k)\right) u(k)+\mathcal{X}_{v r} v(k) r(k) \\
& +\left(\mathcal{X}_{|u| u}+\mathcal{W}_{|u| u}\right) u(k)^{2}+\mathcal{X}_{\mu} \tilde{\tau}_{x}(k)+\mathcal{X}_{\kappa} u(k) \tilde{\tau}_{x}(k)-\mathcal{X}_{u} u_{c}(k)+\mathcal{X}_{|u| u} u_{c}(k)^{2} \\
& +\mathcal{W}_{|u| u} u_{w}(k)^{2}-\mathcal{X}_{v r} v_{c}(k) r(k)-\mathcal{X}_{\kappa} u_{c}(k) \tilde{\tau}_{x}(k) .
\end{aligned}
$$

In this case, the left-hand side of the IV equation when the predictor (5.62) is used can be expressed as

$$
\begin{aligned}
& \bar{E}\left\{\zeta_{u, 4}(k)\left(y_{u}(k)-\varphi_{u, 4}^{T}(k) \theta\right)\right\}=\bar{E}\left\{\zeta _ { u , 4 } ( k ) \left[\left(1+\mathcal{X}_{u}-2 \mathcal{X}_{|u| u}\left(v_{c, N S}(k-1)\right.\right.\right.\right. \\
& \left.\cdot \cos (\psi(k-1))+v_{c, E W}(k-1) \sin (\psi(k-1))\right)-2 \mathcal{W}_{|u| u}\left(v_{w, N S}(k-1) \cos (\psi(k-1))\right. \\
& \left.\left.+v_{w, E W}(k-1) \sin (\psi(k-1))\right)\right) u(k-1)+\mathcal{X}_{v r} v(k-1) r(k-1)+\left(\mathcal{X}_{|u| u}+\mathcal{W}_{|u| u}\right) \\
& \cdot u(k-1)^{2}+\mathcal{X}_{\mu} \tilde{\tau}_{x}(k-1)+\mathcal{X}_{\kappa} u(k-1) \tilde{\tau}_{x}(k-1)-\mathcal{X}_{u}\left(v_{c, N S}(k-1) \cos (\psi(k-1))\right. \\
& \left.+v_{c, E W}(k-1) \sin (\psi(k-1))\right)+\mathcal{X}_{|u| u}\left(v_{c, N S}(k)^{2} \cos ^{2}(\psi(k-1))+2 v_{c, N S}(k-1)\right.
\end{aligned}
$$




$$
\begin{aligned}
& \left.\cdot v_{c, E W}(k-1) \cos (\psi(k-1)) \sin (\psi(k-1))+v_{c, E W}(k-1)^{2} \sin ^{2}(\psi(k-1))\right) \\
& +\mathcal{W}_{|u| u}\left(v_{w, N S}(k)^{2} \cos ^{2}(\psi(k-1))+2 v_{w, N S}(k-1) v_{w, E W}(k-1) \cos (\psi(k-1))\right. \\
& \left.\cdot \sin (\psi(k-1))+v_{w, E W}(k-1)^{2} \sin ^{2}(\psi(k-1))\right)-\mathcal{X}_{v r}\left(-v_{c, N S}(k-1) \sin (\psi(k-1))\right. \\
& \left.+v_{c, E W}(k-1) \cos (\psi(k-1))\right) r(k-1)-\mathcal{X}_{\mathcal{K}}\left(v_{c, N S}(k-1) \cos (\psi(k-1))\right. \\
& \left.+v_{c, E W}(k-1) \sin (\psi(k-1))\right) \tilde{\tau}_{x}(k-1)+e_{u}(k)-\theta_{1}\left(u(k-1)+e_{u}(k-1)\right) \\
& -\theta_{2}\left(v(k-1) r(k-1)+v(k-1) e_{r}(k-1)+r(k-1) e_{v}(k-1)+e_{v}(k-1) e_{r}(k-1)\right) \\
& -\theta_{3}\left(u(k-1)^{2}+2 u(k-1) e_{u}(k-1)+e_{u}(k-1)^{2}\right)-\theta_{4} \tilde{\tau}_{x}(k-1) \\
& -\theta_{5} \tilde{\tau}_{x}(k-1)\left(u(k-1)+e_{u}(k-1)\right)-b_{1} \cos \left(\psi(k-1)+e_{\psi}(k-1)\right) \\
& -b_{2} \sin \left(\psi(k-1)+e_{\psi}(k-1)\right)-b_{3}\left(u(k-1)+e_{u}(k-1)\right) \cos \left(\psi(k-1)+e_{\psi}(k-1)\right) \\
& -b_{4}\left(u(k-1)+e_{u}(k-1)\right) \sin \left(\psi(k-1)+e_{\psi}(k-1)\right)-b_{5}\left(r(k-1)+e_{r}(k-1)\right) \\
& \cdot \cos \left(\psi(k-1)+e_{\psi}(k-1)\right)-b_{6}\left(r(k-1)+e_{r}(k-1)\right) \sin \left(\psi(k-1)+e_{\psi}(k-1)\right) \\
& -b_{7} \tilde{\tau}_{x} \cos \left(\psi(k-1)+e_{\psi}(k-1)\right)-b_{8} \tilde{\tau}_{x} \sin \left(\psi(k-1)+e_{\psi}(k-1)\right) \\
& \left.\left.-b_{9} \cos ^{2}\left(\psi(k-1)+e_{\psi}(k-1)\right)-b_{10} \sin \left(2\left(\psi(k-1)+e_{\psi}(k-1)\right)\right)\right]\right\} \\
& =\left(1+\mathcal{X}_{u}-\theta_{1}\right) \bar{E}\left\{\zeta_{u, 4}(k) u(k-1)\right\}+\left(\mathcal{X}_{v r}-\theta_{2}\right) \bar{E}\left\{\zeta_{u, 4}(k) v(k-1) r(k-1)\right\} \\
& +\left(\mathcal{X}_{|u| u}+\mathcal{W}_{|u| u}-\theta_{3}\right) \bar{E}\left\{\zeta_{u, 4}(k) u(k-1)^{2}\right\}+\left(\mathcal{X}_{\mu}-\theta_{4}\right) \bar{E}\left\{\zeta_{u, 4}(k) \tilde{\tau}_{x}(k-1)\right\} \\
& +\left(\mathcal{X}_{\kappa}-\theta_{5}\right) \bar{E}\left\{\zeta_{u, 4}(k) u(k-1) \tilde{\tau}_{x}(k-1)\right\} \\
& +\left(-\mathcal{X}_{u} \bar{v}_{c, N S}-\rho_{e_{\psi}} b_{1}\right) \bar{E}\left\{\zeta_{u, 4}(k) \cos (\psi(k-1))\right\} \\
& +\left(-\mathcal{X}_{u} \bar{v}_{c, E W}-\rho_{e_{\psi}} b_{2}\right) \bar{E}\left\{\zeta_{u, 4}(k) \sin (\psi(k-1))\right\} \\
& +\left(-2 \mathcal{X}_{|u| u} \bar{v}_{c, N S}-2 \mathcal{W}_{|u| u} \bar{v}_{w, N S}-\rho_{e_{\psi}} b_{3}\right) \bar{E}\left\{\zeta_{u, 4}(k) \cos (\psi(k-1)) u(k-1)\right\} \\
& +\left(-2 \mathcal{X}_{|u| u} \bar{v}_{c, E W}-2 \mathcal{W}_{|u| u} \bar{v}_{w, E W}-\rho_{e_{\psi}} b_{4}\right) \bar{E}\left\{\zeta_{u, 4}(k) \sin (\psi(k-1)) u(k-1)\right\} \\
& +\left(-\mathcal{X}_{v r} \bar{v}_{c, E W}-\rho_{e_{\psi}} b_{5}\right) \bar{E}\left\{\zeta_{u, 4}(k) r(k-1) \cos (\psi(k-1))\right\} \\
& +\left(\mathcal{X}_{v r} \bar{\nu}_{c, N S}-\rho_{e_{\psi}} b_{6}\right) \bar{E}\left\{\zeta_{u, 4}(k) r(k-1) \sin (\psi(k-1))\right\} \\
& +\left(-\mathcal{X}_{\kappa} \bar{\nu}_{c, N S}-\rho_{e_{\psi}} b_{7}\right) \bar{E}\left\{\zeta_{u, 4}(k) \tilde{\tau}_{x}(k-1) \cos (\psi(k-1))\right\} \\
& +\left(-\mathcal{X}_{\kappa} \bar{v}_{c, E W}-\rho_{e_{\psi}} b_{8}\right) \bar{E}\left\{\zeta_{u, 4}(k) \tilde{\tau}_{x}(k-1) \sin (\psi(k-1))\right\}
\end{aligned}
$$




$$
\begin{aligned}
& +\left(\mathcal{X}_{|u| u}\left(\bar{E}\left\{v_{c, N S}(k-1)^{2}\right\}-\bar{E}\left\{v_{c, E W}(k-1)^{2}\right\}\right)+\mathcal{W}_{|u| u}\left(\bar{E}\left\{v_{w, N S}(k-1)^{2}\right\}\right.\right. \\
& \left.\left.-\bar{E}\left\{v_{w, E W}(k-1)^{2}\right\}\right)-\left(2 \sigma_{e_{\psi}}-1\right) b_{9}\right) \bar{E}\left\{\zeta_{u, 4}(k) \cos ^{2}(\psi(k-1))\right\} \\
& +\left(\mathcal{X}_{|u| u} \bar{E}\left\{v_{c, N S}(k-1) v_{c, E W}(k-1)\right\}+\mathcal{W}_{|u| u} \bar{E}\left\{v_{w, N S}(k-1) v_{w, E W}(k-1)\right\}\right. \\
& \left.-\left(2 \sigma_{e_{\psi}}-1\right) b_{10}\right) \bar{E}\left\{\zeta_{u, 4}(k) \sin (2 \psi(k-1))\right\} .
\end{aligned}
$$

From this it can be seen that the asymptotic parameter estimates of (5.63) follow. If the experiments are done such that (5.32) is fulfilled, the sign of terms coming from modulus functions change. However, calculations analogous to the ones in Section 5.1 show that this does not affect the asymptotic parameter estimates.

\section{A.2 Sway equation - without wind measurements}

Under assumption of an experiment design fulfilling (5.68), (5.67) may be rewritten as

$$
\begin{aligned}
v(k+1) & =v(k)+\mathcal{Y}_{u r}\left(u(k)-u_{c}(k)\right) r(k)+\mathcal{Y}_{v}\left(v(k)-v_{c}(k)\right)+\mathcal{Y}_{|v| v}\left(v(k)-v_{c}(k)\right)^{2} \\
& +\mathcal{Y}_{|v| r}\left(v(k)-v_{c}(k)\right) r(k)+\mathcal{W}_{|v| v}\left(v(k)-v_{w}(k)\right)^{2}+\mathcal{Y}_{\mu} \tilde{\tau}_{y}(k) \\
& =\left(1+\mathcal{Y}_{v}-2 \mathcal{Y}_{|v| v} v_{c}(k)-2 \mathcal{W}_{|v| v} v_{w}(k)\right) v(k)+\mathcal{Y}_{u r} u(k) r(k) \\
& +\left(\mathcal{Y}_{|v| v}+\mathcal{W}_{|v| v}\right) v(k)^{2}+\mathcal{Y}_{|v| r} v(k) r(k)+\mathcal{Y}_{\mu} \tilde{\tau}_{y}(k)-\mathcal{Y}_{v} v_{c}(k)+\mathcal{Y}_{|v| v} v_{c}(k)^{2} \\
& +\mathcal{W}_{|v| v} v_{w}(k)^{2}-\mathcal{Y}_{|v| r} v_{c}(k) r(k)-\mathcal{Y}_{u r} u_{c}(k) r(k) .
\end{aligned}
$$

In this case, the left-hand side of the IV equation when the predictor (5.71) is used can be expressed as

$$
\begin{aligned}
& \bar{E}\left\{\zeta_{v, 1}(k)\left(y_{v}(k)-\varphi_{v, 1}^{T}(k) \theta\right)\right\}=\bar{E}\left\{\zeta _ { v , 1 } ( k ) \left[\left(1+\mathcal{Y}_{v}-2 \mathcal{Y}_{|v| v}\left(-v_{c, N S}(k-1)\right.\right.\right.\right. \\
& \left.\cdot \sin (\psi(k-1))+v_{c, E W}(k-1) \cos (\psi(k-1))\right)-2 \mathcal{W}_{|u| u}\left(-v_{w, N S}(k-1)\right. \\
& \left.\left.\cdot \sin (\psi(k-1))+v_{w, E W}(k-1) \cos (\psi(k-1))\right)\right) v(k-1)+\mathcal{Y}_{u r} u(k-1) r(k-1) \\
& +\left(\mathcal{Y}_{|v| v}+\mathcal{W}_{|v| v}\right) v(k-1)^{2}+\mathcal{Y}_{|v| r} v(k-1) r(k-1)+\mathcal{Y}_{\mu} \tilde{\tau}_{y}(k-1)-\mathcal{Y}_{v}\left(-v_{c, N S}(k-1)\right. \\
& \left.\cdot \sin (\psi(k-1))+v_{c, E W}(k-1) \cos (\psi(k-1))\right)+\mathcal{Y}_{|v| v}\left(v_{c, N S}(k)^{2} \sin ^{2}(\psi(k-1))\right. \\
& \left.-2 v_{c, N S}(k-1) v_{c, E W}(k-1) \cos (\psi(k-1)) \sin (\psi(k-1))+v_{c, E W}(k-1)^{2} \cos ^{2}(\psi(k-1))\right) \\
& +\mathcal{W}_{|v| v}\left(v_{w, N S}(k-1)^{2} \sin ^{2}(\psi(k-1))-2 v_{w, N S}(k-1) v_{w, E W}(k-1) \cos (\psi(k-1))\right. \\
& \left.\cdot \sin (\psi(k-1))+v_{w, E W}(k-1)^{2} \cos ^{2}(\psi(k-1))\right)-\mathcal{Y}_{|v| r}\left(-v_{c, N S}(k-1)\right.
\end{aligned}
$$




$$
\begin{aligned}
& \left.\cdot \sin (\psi(k-1))+v_{c, E W}(k-1) \cos (\psi(k-1))\right) r(k-1)-\mathcal{Y}_{u r}\left(v_{c, N S}(k-1)\right. \\
& \left.\cdot \cos (\psi(k-1))+v_{c, E W}(k-1) \sin (\psi(k-1))\right) r(k-1)+e_{v}(k) \\
& -\theta_{1}\left(v(k-1)+e_{v}(k-1)\right) \\
& -\theta_{2}\left(u(k-1) r(k-1)+u(k-1) e_{r}(k-1)+r(k-1) e_{u}(k-1)+e_{u}(k-1) e_{r}(k-1)\right) \\
& -\theta_{3}\left(v(k-1)^{2}+2 v(k-1) e_{v}(k-1)+e_{v}(k-1)^{2}\right) \\
& -\theta_{4}\left(v(k-1) r(k-1)+v(k-1) e_{r}(k-1)+r(k-1) e_{v}(k-1)+e_{v}(k-1) e_{r}(k-1)\right) \\
& -\theta_{5} \tilde{\tau}_{y}(k-1)-b_{1} \cos \left(\psi(k-1)+e_{\psi}(k-1)\right)-b_{2} \sin \left(\psi(k-1)+e_{\psi}(k-1)\right) \\
& -b_{3}\left(u(k-1)+e_{u}(k-1)\right) \cos \left(\psi(k-1)+e_{\psi}(k-1)\right) \\
& -b_{4}\left(u(k-1)+e_{u}(k-1)\right) \sin \left(\psi(k-1)+e_{\psi}(k-1)\right) \\
& -b_{5}\left(r(k-1)+e_{r}(k-1)\right) \cos \left(\psi(k-1)+e_{\psi}(k-1)\right) \\
& -b_{6}\left(r(k-1)+e_{r}(k-1)\right) \sin \left(\psi(k-1)+e_{\psi}(k-1)\right) \\
& \left.\left.-b_{7} \cos ^{2}\left(\psi(k-1)+e_{\psi}(k-1)\right)-b_{8} \sin \left(2\left(\psi(k-1)+e_{\psi}(k-1)\right)\right)\right]\right\} \\
& =\left(1+\mathcal{Y}_{v}-\theta_{1}\right) \bar{E}\left\{\zeta_{v, 1}(k) v(k-1)\right\}+\left(\mathcal{Y}_{u r}-\theta_{2}\right) \bar{E}\left\{\zeta_{v, 1}(k) u(k-1) r(k-1)\right\} \\
& +\left(\mathcal{Y}_{|v| v}+\mathcal{W}_{|v| v}-\theta_{3}\right) \bar{E}\left\{\zeta_{v, 1}(k) v(k-1)^{2}\right\}+\left(\mathcal{Y}_{|v| r}-\theta_{4}\right) \bar{E}\left\{\zeta_{v, 1}(k) v(k-1) r(k-1)\right\} \\
& +\left(\mathcal{Y}_{\mu}-\theta_{5}\right) \bar{E}\left\{\zeta_{v, 1}(k) \tilde{\tau}_{y}(k-1)\right\} \\
& +\left(-\mathcal{Y}_{v} \bar{v}_{c, E W}-\rho_{e_{\psi}} b_{1}\right) \bar{E}\left\{\zeta_{v, 1}(k) \cos (\psi(k-1))\right\} \\
& +\left(\mathcal{Y}_{v} \bar{\nu}_{c, N S}-\rho_{e_{\psi}} b_{2}\right) \bar{E}\left\{\zeta_{v, 1}(k) \sin (\psi(k-1))\right\} \\
& +\left(-2 \mathcal{Y}_{|v| v} \bar{v}_{c, E W}-2 \mathcal{W}_{|v| v} \bar{v}_{w, E W}-\rho_{e_{\psi}} b_{3}\right) \bar{E}\left\{\zeta_{v, 1}(k) \cos (\psi(k-1)) v(k-1)\right\} \\
& +\left(2 \mathcal{Y}_{|v| v} \bar{v}_{c, N S}+2 \mathcal{W}_{|v| v} \bar{\nu}_{w, N S}-\rho_{e_{\psi}} b_{4}\right) \bar{E}\left\{\zeta_{v, 1}(k) \sin (\psi(k-1)) v(k-1)\right\} \\
& +\left(-\mathcal{Y}_{|v| r} \bar{v}_{c, E W}-\mathcal{Y}_{u r} \bar{v}_{c, N S}-\rho_{e_{\psi}} b_{5}\right) \bar{E}\left\{\zeta_{v, 1}(k) r(k-1) \cos (\psi(k-1))\right\} \\
& +\left(\mathcal{Y}_{|v| r} \bar{\nu}_{c, N S}-\mathcal{Y}_{u r} \bar{\nu}_{c, E W}-\rho_{e_{\psi}} b_{6}\right) \bar{E}\left\{\zeta_{v, 1}(k) r(k-1) \sin (\psi(k-1))\right\} \\
& +\left(\mathcal{Y}_{|v| v}\left(\bar{E}\left\{v_{c, E W}(k-1)^{2}\right\}-\bar{E}\left\{v_{c, N S}(k-1)^{2}\right\}\right)+\mathcal{W}_{|v| v}\left(\bar{E}\left\{v_{w, E W}(k-1)^{2}\right\}\right.\right. \\
& \left.\left.-\bar{E}\left\{v_{w, N S}(k-1)^{2}\right\}\right)-\left(2 \sigma_{e_{\psi}}-1\right) b_{7}\right) \bar{E}\left\{\zeta_{v, 1}(k) \cos ^{2}(\psi(k-1))\right\} \\
& +\left(-\mathcal{Y}_{|v| v} \bar{E}\left\{v_{c, N S}(k-1) v_{c, E W}(k-1)\right\}-\mathcal{W}_{|v| v} \bar{E}\left\{v_{w, N S}(k-1) v_{w, E W}(k-1)\right\}\right.
\end{aligned}
$$




$$
\left.-\left(2 \sigma_{e_{\psi}}-1\right) b_{8}\right) \bar{E}\left\{\zeta_{v, 1}(k) \sin (2 \psi(k-1))\right\}
$$

From this it can be seen that the asymptotic parameter estimates of (5.72) follow. If the experiments are done such that (5.69) is fulfilled, the sign of terms coming from modulus functions change. Calculations analogous to the ones in Section 5.1 show that this does not affect the asymptotic parameter estimates.

\section{A.3 Sway equation - with wind measurements}

Under assumption of an experiment design fulfilling (5.77), (5.67) may be rewritten as

$$
\begin{aligned}
v(k+1) & =v(k)+\mathcal{Y}_{u r}\left(u(k)-u_{c}(k)\right) r(k)+\mathcal{Y}_{v}\left(v(k)-v_{c}(k)\right)+\mathcal{Y}_{|v| v}\left(v(k)-v_{c}(k)\right)^{2} \\
& +\mathcal{Y}_{|v| r}\left(v(k)-v_{c}(k)\right) r(k)+\mathcal{W}_{|v| v} v_{q}(k)^{2}+\mathcal{Y}_{\mu} \tilde{\tau}_{y}(k) \\
& =\left(1+\mathcal{Y}_{v}-2 \mathcal{Y}_{|v| v} v_{c}(k)\right) v(k)+\mathcal{Y}_{u r} u(k) r(k)+\mathcal{Y}_{|v| v} v(k)^{2} \\
& +\mathcal{Y}_{|v| r} v(k) r(k)+\mathcal{W}_{|v| v} v_{q}(k)^{2}+\mathcal{Y}_{\mu} \tilde{\tau}_{y}(k)-\mathcal{Y}_{v} v_{c}(k) \\
& +\mathcal{Y}_{|v| v} v_{c}(k)^{2}-\mathcal{Y}_{|v| r} v_{c}(k) r(k)-\mathcal{Y}_{u r} u_{c}(k) r(k) .
\end{aligned}
$$

In this case, the left-hand side of the IV equation when the predictor (5.76) is used can be expressed as

$$
\begin{aligned}
& \bar{E}\left\{\zeta_{v, 2}(k)\left(y_{v}(k)-\varphi_{v, 2}^{T}(k) \theta\right)\right\}=\bar{E}\left\{\zeta _ { v , 2 } ( k ) \left[\left(1+\mathcal{Y}_{v}-2 \mathcal{Y}_{|v| v}\left(-v_{c, N S}(k-1)\right.\right.\right.\right. \\
& \left.\left.\cdot \sin (\psi(k-1))+v_{c, E W}(k-1) \cos (\psi(k-1))\right)\right) v(k-1)+\mathcal{Y}_{u r} u(k-1) r(k-1) \\
& +\mathcal{Y}_{|v| v} v(k-1)^{2}+\mathcal{Y}_{|v| r} v(k-1) r(k-1)+\mathcal{W}_{|v| v} v_{q}(k-1)^{2}+\mathcal{Y}_{\mu} \tilde{\tau}_{y}(k-1) \\
& -\mathcal{Y}_{v}\left(-v_{c, N S}(k-1) \sin (\psi(k-1))+v_{c, E W}(k-1) \cos (\psi(k-1))\right) \\
& +\mathcal{Y}_{|v| v}\left(v_{c, N S}(k)^{2} \sin ^{2}(\psi(k-1))-2 v_{c, N S}(k-1) v_{c, E W}(k-1) \cos (\psi(k-1))\right. \\
& \left.\cdot \sin (\psi(k-1))+v_{c, E W}(k-1)^{2} \cos ^{2}(\psi(k-1))\right)-\mathcal{Y}_{|v| r}\left(-v_{c, N S}(k-1)\right. \\
& \left.\cdot \sin (\psi(k-1))+v_{c, E W}(k-1) \cos (\psi(k-1))\right) r(k-1)-\mathcal{Y}_{u r}\left(v_{c, N S}(k-1)\right. \\
& \left.\cdot \cos (\psi(k-1))+v_{c, E W}(k-1) \sin (\psi(k-1))\right) r(k-1)+e_{v}(k) \\
& -\theta_{1}\left(v(k-1)+e_{v}(k-1)\right) \\
& -\theta_{2}\left(u(k-1) r(k-1)+u(k-1) e_{r}(k-1)+r(k-1) e_{u}(k-1)+e_{u}(k-1) e_{r}(k-1)\right) \\
& -\theta_{3}\left(v(k-1)^{2}+2 v(k-1) e_{v}(k-1)+e_{v}(k-1)^{2}\right) \\
& -\theta_{4}\left(v(k-1) r(k-1)+v(k-1) e_{r}(k-1)+r(k-1) e_{v}(k-1)+e_{v}(k-1) e_{r}(k-1)\right)
\end{aligned}
$$




$$
\begin{aligned}
& -\theta_{5}\left(v_{q}(k-1)^{2}+2 v_{q}(k-1) e_{v_{q}}(k-1)+e_{v_{q}}(k-1)^{2}\right) \\
& -\theta_{6} \tilde{\tau}_{y}(k-1)-b_{1} \cos \left(\psi(k-1)+e_{\psi}(k-1)\right)-b_{2} \sin \left(\psi(k-1)+e_{\psi}(k-1)\right) \\
& -b_{3}\left(u(k-1)+e_{u}(k-1)\right) \cos \left(\psi(k-1)+e_{\psi}(k-1)\right) \\
& -b_{4}\left(u(k-1)+e_{u}(k-1)\right) \sin \left(\psi(k-1)+e_{\psi}(k-1)\right) \\
& -b_{5}\left(r(k-1)+e_{r}(k-1)\right) \cos \left(\psi(k-1)+e_{\psi}(k-1)\right) \\
& -b_{6}\left(r(k-1)+e_{r}(k-1)\right) \sin \left(\psi(k-1)+e_{\psi}(k-1)\right) \\
& \left.\left.-b_{7} \cos ^{2}\left(\psi(k-1)+e_{\psi}(k-1)\right)-b_{8} \sin \left(2\left(\psi(k-1)+e_{\psi}(k-1)\right)\right)\right]\right\} \\
& =\left(1+\mathcal{Y}_{v}-\theta_{1}\right) \bar{E}\left\{\zeta_{v, 2}(k) v(k-1)\right\}+\left(\mathcal{Y}_{u r}-\theta_{2}\right) \bar{E}\left\{\zeta_{v, 2}(k) u(k-1) r(k-1)\right\} \\
& +\left(\mathcal{Y}_{|v| v}-\theta_{3}\right) \bar{E}\left\{\zeta_{v, 2}(k) v(k-1)^{2}\right\}+\left(\mathcal{Y}_{|v| r}-\theta_{4}\right) \bar{E}\left\{\zeta_{v, 2}(k) v(k-1) r(k-1)\right\} \\
& +\left(\mathcal{W}_{|v| v}-\theta_{5}\right) \bar{E}\left\{\zeta_{v, 2}(k) v_{q}(k-1)^{2}\right\} \\
& +\left(\mathcal{Y}_{\mu}-\theta_{6}\right) \bar{E}\left\{\zeta_{v, 2}(k) \tilde{\tau}_{y}(k-1)\right\}+\left(-\mathcal{Y}_{v} \bar{\nu}_{c, E W}-\rho_{e_{\psi}} b_{1}\right) \bar{E}\left\{\zeta_{v, 2}(k) \cos (\psi(k-1))\right\} \\
& +\left(\mathcal{Y}_{v} \bar{v}_{c, N S}-\rho_{e_{\psi}} b_{2}\right) \bar{E}\left\{\zeta_{v, 2}(k) \sin (\psi(k-1))\right\} \\
& +\left(-2 \mathcal{Y}_{|v| v} \bar{v}_{c, E W}-\rho_{e_{\psi}} b_{3}\right) \bar{E}\left\{\zeta_{v, 2}(k) \cos (\psi(k-1)) v(k-1)\right\} \\
& +\left(2 \mathcal{Y}_{|v| v} \bar{\nu}_{c, N S}-\rho_{e_{\psi}} b_{4}\right) \bar{E}\left\{\zeta_{v, 2}(k) \sin (\psi(k-1)) v(k-1)\right\} \\
& +\left(-\mathcal{Y}_{|v| r} \bar{\nu}_{c, E W}-\mathcal{Y}_{u r} \bar{\nu}_{c, N S}-\rho_{e_{\psi}} b_{5}\right) \bar{E}\left\{\zeta_{v, 2}(k) r(k-1) \cos (\psi(k-1))\right\} \\
& +\left(\mathcal{Y}_{|v| r} \bar{v}_{c, N S}-\mathcal{Y}_{u r} \bar{v}_{c, E W}-\rho_{e_{\psi}} b_{6}\right) \bar{E}\left\{\zeta_{v, 2}(k) r(k-1) \sin (\psi(k-1))\right\} \\
& +\left(\mathcal{Y}_{|v| v}\left(\bar{E}\left\{v_{c, E W}(k-1)^{2}-v_{c, N S}(k-1)^{2}\right\}\right)-\left(2 \sigma_{e_{\psi}}-1\right) b_{7}\right) \bar{E}\left\{\zeta_{v, 2}(k) \cos ^{2}(\psi(k-1))\right\} \\
& +\left(-\mathcal{Y}_{|v| v} \bar{E}\left\{v_{c, N S}(k-1) v_{c, E W}(k-1)\right\}-\left(2 \sigma_{e_{\psi}}-1\right) b_{8}\right) \bar{E}\left\{\zeta_{v, 2}(k) \sin (2 \psi(k-1))\right\} \text {. }
\end{aligned}
$$

From (5.96) it can be seen that the asymptotic parameter estimates of (5.81) follow. If the experiments are done such that (5.80) is fulfilled, the signs of terms coming from modulus functions change. Calculations analogous to the ones in Section 5.1 show that this does not affect the asymptotic parameter estimates. If the relative wind-velocity component $v_{q}(k)$ is of opposite sign to its absolutevelocity counterpart $v(k)$, as in (5.78) and (5.79), the sign of the aerodynamic term changes. This does not affect the asymptotic parameter estimates either. 


\section{A.4 Yaw-rate equation - without wind measurements}

Under assumption of an experiment design fulfilling (5.83), (5.82) may be rewritten as

$$
\begin{aligned}
r(k+1) & =r(k)+\mathcal{N}_{u v}\left(u(k)-u_{c}(k)\right)\left(v(k)-v_{c}(k)\right)+\mathcal{N}_{r} r(k)+\mathcal{N}_{|v| v}\left(v(k)-v_{c}(k)\right)^{2} \\
& +\mathcal{N}_{|v| r}\left(v(k)-v_{c}(k)\right) r(k)+\mathcal{W}_{u v}\left(u(k)-u_{w}(k)\right)\left(v(k)-v_{w}(k)\right)+\mathcal{N}_{\mu} \tilde{\tau}_{\psi}(k) \\
& =\left(1+\mathcal{N}_{r}-\mathcal{N}_{|v| r} v_{c}(k)\right) r(k)+\left(\mathcal{N}_{u v}+\mathcal{W}_{u v}\right) u(k) v(k)+\mathcal{N}_{|v| v} v(k)^{2} \\
& +\mathcal{N}_{|v| r} v(k) r(k)+\mathcal{N}_{\mu} \tilde{\tau}_{\psi}(k)-\left(\mathcal{N}_{u v} u_{c}(k)+2 \mathcal{N}_{|v| v} v_{c}(k)+\mathcal{W}_{u v} u_{w}(k)\right) v(k) \\
& -\left(\mathcal{N}_{u v} v_{c}(k)+\mathcal{W}_{u v} v_{w}(k)\right) u(k)+\mathcal{N}_{|v| v} v_{c}(k)^{2}+\mathcal{N}_{u v} u_{c}(k) v_{c}(k) \\
& +\mathcal{W}_{u v} u_{w}(k) v_{w}(k) .
\end{aligned}
$$

In this case, the left-hand side of the IV equation when the predictor (5.86) is used can be expressed as

$$
\begin{aligned}
& \bar{E}\left\{\zeta_{r, 1}(k)\left(y_{r}(k)-\varphi_{r, 1}^{T}(k) \theta\right)\right\}=\bar{E}\left\{\zeta _ { r , 1 } ( k ) \left[\left(1+\mathcal{N}_{r}-\mathcal{N}_{|v| r}\left(-v_{c, N S}(k-1)\right.\right.\right.\right. \\
& \left.\left.\cdot \sin (\psi(k-1))+v_{c, E W}(k-1) \cos (\psi(k-1))\right)\right) r(k-1)+\left(\mathcal{N}_{u v}+\mathcal{W}_{u v}\right) u(k-1) \\
& \cdot v(k-1)+\mathcal{N}_{|v| v} v(k-1)^{2}+\mathcal{N}_{|v| r} v(k-1) r(k-1)+\mathcal{N}_{\mu} \tilde{\tau}_{\psi}(k-1)-\left(\mathcal { N } _ { u v } \left(v_{c, N S}(k-1)\right.\right. \\
& \left.\cdot \cos (\psi(k-1))+v_{c, E W}(k-1) \sin (\psi(k-1))\right)+2 \mathcal{N}_{|v| v}\left(-v_{c, N S}(k-1)\right. \\
& \left.\cdot \sin (\psi(k-1))+v_{c, E W}(k-1) \cos (\psi(k-1))\right)+\mathcal{W}_{u v}\left(v_{w, N S}(k-1)\right. \\
& \left.\left.\cdot \cos (\psi(k-1))+v_{w, E W}(k-1) \sin (\psi(k-1))\right)\right) v(k-1)-\left(\mathcal { N } _ { u v } \left(-v_{c, N S}(k-1)\right.\right. \\
& \left.\cdot \sin (\psi(k-1))+v_{c, E W}(k-1) \cos (\psi(k-1))\right)+\mathcal{W}_{u v}\left(-v_{w, N S}(k-1) \sin (\psi(k-1))\right. \\
& \left.\left.+v_{w, E W}(k-1) \cos (\psi(k-1))\right)\right) u(k-1)+\mathcal{N}_{|v| v}\left(v_{c, N S}(k-1)^{2} \sin ^{2}(\psi(k-1))\right. \\
& \left.-2 v_{c, N S}(k-1) v_{c, E W}(k-1) \cos (\psi(k-1)) \sin (\psi(k-1))+v_{c, E W}(k-1)^{2} \cos ^{2}(\psi(k-1))\right) \\
& +\mathcal{N}_{u v}\left(-v_{c, N S}(k-1) v_{c, E W}(k-1) \sin ^{2}(\psi(k-1))-\left(v_{c, N S}(k-1)^{2}-v_{c, E W}(k-1)^{2}\right)\right. \\
& \left.\cdot \cos (\psi(k-1)) \sin (\psi(k-1))+v_{c, N S}(k-1) v_{c, E W}(k-1) \cos ^{2}(\psi(k-1))\right) \\
& +\mathcal{W}_{u v}\left(-v_{w, N S}(k-1) v_{w, E W}(k-1) \sin ^{2}(\psi(k-1))-\left(v_{w, N S}(k-1)^{2}-v_{w, E W}(k-1)^{2}\right)\right. \\
& \left.\cdot \cos (\psi(k-1)) \sin (\psi(k-1))+v_{w, N S}(k-1) v_{w, E W}(k-1) \cos ^{2}(\psi(k-1))\right) \\
& +e_{r}(k)-\theta_{1}\left(r(k-1)+e_{r}(k-1)\right)
\end{aligned}
$$




$$
\begin{aligned}
& -\theta_{2}\left(u(k-1) v(k-1)+u(k-1) e_{v}(k-1)+v(k-1) e_{u}(k-1)+e_{u}(k-1) e_{v}(k-1)\right) \\
& -\theta_{3}\left(v(k-1)^{2}+2 v(k-1) e_{v}(k-1)+e_{v}(k-1)^{2}\right) \\
& -\theta_{4}\left(v(k-1) r(k-1)+v(k-1) e_{r}(k-1)+r(k-1) e_{v}(k-1)+e_{v}(k-1) e_{r}(k-1)\right) \\
& -\theta_{5} \tilde{\tau}_{\psi}(k-1)-b_{1}\left(r(k-1)+e_{r}(k-1)\right) \cos \left(\psi(k-1)+e_{\psi}(k-1)\right) \\
& -b_{2}\left(r(k-1)+e_{r}(k-1)\right) \sin \left(\psi(k-1)+e_{\psi}(k-1)\right) \\
& -v_{3}\left(v(k-1)+e_{v}(k-1)\right) \cos \left(\psi(k-1)+e_{\psi}(k-1)\right) \\
& -b_{4}\left(v(k-1)+e_{v}(k-1)\right) \sin \left(\psi(k-1)+e_{\psi}(k-1)\right) \\
& -b_{5}\left(u(k-1)+e_{u}(k-1)\right) \cos \left(\psi(k-1)+e_{\psi}(k-1)\right) \\
& -b_{6}\left(u(k-1)+e_{u}(k-1)\right) \sin \left(\psi(k-1)+e_{\psi}(k-1)\right) \\
& \left.\left.-b_{7} \cos ^{2}\left(\psi(k-1)+e_{\psi}(k-1)\right)-b_{8} \sin \left(2\left(\psi(k-1)+e_{\psi}(k-1)\right)\right)\right]\right\} \\
& =\left(1+\mathcal{N}_{r}-\theta_{1}\right) \bar{E}\left\{\zeta_{r, 1}(k) r(k-1)\right\}+\left(\mathcal{N}_{u v}+\mathcal{W}_{u v}-\theta_{2}\right) \bar{E}\left\{\zeta_{r, 1}(k) u(k-1) v(k-1)\right\} \\
& +\left(\mathcal{N}_{|v| v}-\theta_{3}\right) \bar{E}\left\{\zeta_{r, 1}(k) v(k-1)^{2}\right\}+\left(\mathcal{N}_{|v| r}-\theta_{4}\right) \bar{E}\left\{\zeta_{r, 1}(k) v(k-1) r(k-1)\right\} \\
& +\left(\mathcal{N}_{\mu}-\theta_{5}\right) \bar{E}\left\{\zeta_{r, 1}(k) \tilde{\tau}_{\psi}(k-1)\right\} \\
& +\left(-\mathcal{N}_{|v| r} \bar{v}_{c, E W}-\rho_{e_{\psi}} b_{1}\right) \bar{E}\left\{\zeta_{r, 1}(k) r(k-1) \cos (\psi(k-1))\right\} \\
& +\left(\mathcal{N}_{|v| r} \bar{v}_{c, N S}-\rho_{e_{\psi}} b_{2}\right) \bar{E}\left\{\zeta_{r, 1}(k) r(k-1) \sin (\psi(k-1))\right\} \\
& +\left(-\mathcal{N}_{u v} \bar{v}_{c, N S}-2 \mathcal{N}_{|v| v} \bar{v}_{c, E W}-\mathcal{W}_{u v} \bar{v}_{w, N S}-\rho_{e_{\psi}} b_{3}\right) \bar{E}\left\{\zeta_{r, 1}(k) v(k-1) \cos (\psi(k-1))\right\} \\
& +\left(-\mathcal{N}_{u v} \bar{v}_{c, E W}+2 \mathcal{N}_{|v| v} \bar{v}_{c, N S}-\mathcal{W}_{u v} \bar{v}_{w, E W}-\rho_{e_{\psi}} b_{4}\right) \bar{E}\left\{\zeta_{r, 1}(k) v(k-1) \sin (\psi(k-1))\right\} \\
& +\left(-\mathcal{N}_{u v} \bar{v}_{c, E W}-\mathcal{W}_{u v} \bar{v}_{w, E W}-\rho_{e_{\psi}} b_{5}\right) \bar{E}\left\{\zeta_{r, 1}(k) u(k-1) \cos (\psi(k-1))\right\} \\
& +\left(\mathcal{N}_{u v} \bar{v}_{c, N S}+\mathcal{W}_{u v} \bar{v}_{w, N S}-\rho_{e_{\psi}} b_{6}\right) \bar{E}\left\{\zeta_{r, 1}(k) u(k-1) \sin (\psi(k-1))\right\} \\
& +\left(\mathcal{N}_{|v| v} \bar{E}\left\{v_{c, E W}(k-1)^{2}-v_{c, N S}(k-1)^{2}\right\}+2 \mathcal{N}_{u v} \bar{E}\left\{v_{c, N S}(k-1) v_{c, E W}(k-1)\right\}\right. \\
& \left.+2 \mathcal{W}_{u v} \bar{E}\left\{v_{w, N S}(k-1) v_{w, E W}(k-1)\right\}-\left(2 \sigma_{e_{\psi}}-1\right) b_{7}\right) \bar{E}\left\{\zeta_{r, 1}(k) \cos ^{2}(\psi(k-1))\right\} \\
& +\left(-\mathcal{N}_{|v| v} \bar{E}\left\{v_{c, N S}(k-1) v_{c, E W}(k-1)\right\}+\frac{1}{2} \mathcal{N}_{u v} \bar{E}\left\{v_{c, E W}(k-1)^{2}-v_{c, N S}(k-1)^{2}\right\}\right. \\
& \left.+\frac{1}{2} \mathcal{W}_{u v} \bar{E}\left\{v_{w, E W}(k-1)^{2}-v_{w, N S}(k-1)^{2}\right\}-\left(2 \sigma_{e_{\psi}}-1\right) b_{8}\right) \bar{E}\left\{\zeta_{r, 1}(k) \sin (2 \psi(k-1))\right\} \text {. }
\end{aligned}
$$


From this it can be seen that the asymptotic parameter estimates

$$
\lim _{N \rightarrow \infty} \hat{\theta}_{N}^{I V}=\left[\begin{array}{c}
1+\mathcal{N}_{r} \\
\mathcal{N}_{u v}+\mathcal{W}_{u v} \\
\mathcal{N}_{|v| v} \\
\mathcal{N}_{|v| r} \\
\mathcal{N}_{\mu} \\
-\frac{1}{\rho_{e_{\psi}}} \mathcal{N}_{|v| r} \bar{v}_{c, E W} \\
\frac{1}{\rho_{e_{\psi}}} \mathcal{N}_{|v| r} \bar{v}_{c, N S} \\
\frac{1}{\rho_{e_{\psi}}}\left(-\mathcal{N}_{u v} \bar{v}_{c, N S}-2 \mathcal{N}_{|v| v} \bar{v}_{c, E W}-\mathcal{W}_{u v} \bar{v}_{w, N S}\right) \\
\frac{1}{\rho_{e_{\psi}}}\left(-\mathcal{N}_{u v} \bar{v}_{c, E W}+2 \mathcal{N}_{|v| v} \bar{v}_{c, N S}-\mathcal{W}_{u v} \bar{v}_{w, E W}\right) \\
-\frac{1}{\rho_{e_{\psi}}}\left(\mathcal{N}_{u v} \bar{v}_{c, E W}+\mathcal{W}_{u v} \bar{v}_{w, E W}\right) \\
\frac{1}{\rho_{e_{\psi}}}\left(\mathcal{N}_{u v} \bar{v}_{c, N S}+\mathcal{W}_{u v} \bar{v}_{w, N S}\right) \\
\frac{1}{2 \sigma_{e_{\psi}}-1} \xi_{5}\left(\nu_{c, n}, v_{w, n}\right) \\
\frac{1}{2 \sigma_{e_{\psi}}-1} \xi_{6}\left(\nu_{c, n}, v_{w, n}\right)
\end{array}\right],
$$

follow. Here

$$
\begin{aligned}
& \xi_{5}\left(v_{c, n}, v_{w, n}\right)=\mathcal{N}_{|v| v}\left(\bar{E}\left\{v_{c, E W}(k-1)^{2}\right\}-\bar{E}\left\{v_{c, N S}(k-1)^{2}\right\}\right) \\
& +2\left(\mathcal{N}_{u v} \bar{E}\left\{v_{c, N S}(k-1) v_{c, E W}(k-1)\right\}+\mathcal{W}_{u v} \bar{E}\left\{v_{w, N S}(k-1) v_{w, E W}(k-1)\right\}\right), \\
& \xi_{6}\left(v_{c, n}, v_{w, n}\right)=-\mathcal{N}_{|v| v} \bar{E}\left\{v_{c, N S}(k-1) v_{c, E W}(k-1)\right\} \\
& +\frac{1}{2}\left(\mathcal{N}_{u v} \bar{E}\left\{v_{c, E W}(k-1)^{2}-v_{c, N S}(k-1)^{2}\right\}+\mathcal{W}_{u v} \bar{E}\left\{v_{w, E W}(k-1)^{2}-v_{w, N S}(k-1)^{2}\right\}\right) .
\end{aligned}
$$

If the experiments are done such that (5.84) is fulfilled, (5.82) may be rewritten as

$$
\begin{aligned}
r(k+1) & =r(k)+\mathcal{N}_{u v}\left(u(k)-u_{c}(k)\right)\left(v(k)-v_{c}(k)\right)+\mathcal{N}_{r} r(k)-\mathcal{N}_{|v| v}\left(v(k)-v_{c}(k)\right)^{2} \\
& -\mathcal{N}_{|v| r}\left(v(k)-v_{c}(k)\right) r(k)+\mathcal{W}_{u v}\left(u(k)-u_{w}(k)\right)\left(v(k)-v_{w}(k)\right)+\mathcal{N}_{\mu} \tilde{\tau}_{\psi}(k) \\
& =\left(1+\mathcal{N}_{r}+\mathcal{N}_{|v| r} v_{c}(k)\right) r(k)+\left(\mathcal{N}_{u v}+\mathcal{W}_{u v}\right) u(k) v(k)-\mathcal{N}_{|v| v} v(k)^{2} \\
& -\mathcal{N}_{|v| r} v(k) r(k)+\mathcal{N}_{\mu} \tilde{\tau}_{\psi}(k)-\left(\mathcal{N}_{u v} u_{c}(k)-2 \mathcal{N}_{|v| v} v_{c}(k)+\mathcal{W}_{u v} u_{w}(k)\right) v(k) \\
& -\left(\mathcal{N}_{u v} v_{c}(k)+\mathcal{W}_{u v} v_{w}(k)\right) u(k)-\mathcal{N}_{|v| v} v_{c}(k)^{2}+\mathcal{N}_{u v} u_{c}(k) v_{c}(k) \\
& +\mathcal{W}_{u v} u_{w}(k) v_{w}(k) .
\end{aligned}
$$

In this case, the left-hand side of the IV equation can be expressed as $\bar{E}\left\{\zeta_{r, 1}(k)\left(y_{r}(k)-\varphi_{r, 1}^{T}(k) \theta\right)\right\}=\bar{E}\left\{\zeta_{r, 1}(k)\left[\left(1+\mathcal{N}_{r}+\mathcal{N}_{|v| r}\left(-v_{c, N S}(k-1)\right.\right.\right.\right.$ 


$$
\begin{aligned}
& \left.\left.\cdot \sin (\psi(k-1))+v_{c, E W}(k-1) \cos (\psi(k-1))\right)\right) r(k-1)+\left(\mathcal{N}_{u v}+\mathcal{W}_{u v}\right) u(k-1) \\
& \cdot v(k-1)-\mathcal{N}_{|v| v} v(k-1)^{2}-\mathcal{N}_{|v| r} v(k-1) r(k-1)+\mathcal{N}_{\mu} \tilde{\tau}_{\psi}(k-1)-\left(\mathcal { N } _ { u v } \left(v_{c, N S}(k-1)\right.\right. \\
& \left.\cdot \cos (\psi(k-1))+v_{c, E W}(k-1) \sin (\psi(k-1))\right)-2 \mathcal{N}_{|v| v}\left(-v_{c, N S}(k-1)\right. \\
& \left.\cdot \sin (\psi(k-1))+v_{c, E W}(k-1) \cos (\psi(k-1))\right)+\mathcal{W}_{u v}\left(v_{w, N S}(k-1)\right. \\
& \left.\left.\cdot \cos (\psi(k-1))+v_{w, E W}(k-1) \sin (\psi(k-1))\right)\right) v(k-1)-\left(\mathcal { N } _ { u v } \left(-v_{c, N S}(k-1)\right.\right. \\
& \left.\cdot \sin (\psi(k-1))+v_{c, E W}(k-1) \cos (\psi(k-1))\right)+\mathcal{W}_{u v}\left(-v_{w, N S}(k-1) \sin (\psi(k-1))\right. \\
& \left.\left.+v_{w, E W}(k-1) \cos (\psi(k-1))\right)\right) u(k-1)-\mathcal{N}_{|v| v}\left(v_{c, N S}(k-1)^{2} \sin ^{2}(\psi(k-1))\right. \\
& \left.-2 v_{c, N S}(k-1) v_{c, E W}(k-1) \cos (\psi(k-1)) \sin (\psi(k-1))+v_{c, E W}(k-1)^{2} \cos ^{2}(\psi(k-1))\right) \\
& +\mathcal{N}_{u v}\left(-v_{c, N S}(k-1) v_{c, E W}(k-1) \sin ^{2}(\psi(k-1))-\left(v_{c, N S}(k-1)^{2}-v_{c, E W}(k-1)^{2}\right)\right. \\
& \left.\cdot \cos (\psi(k-1)) \sin (\psi(k-1))+v_{c, N S}(k-1) v_{c, E W}(k-1) \cos ^{2}(\psi(k-1))\right) \\
& +\mathcal{W}_{u v}\left(-v_{w, N S}(k-1) v_{w, E W}(k-1) \sin ^{2}(\psi(k-1))-\left(v_{w, N S}(k-1)^{2}-v_{w, E W}(k-1)^{2}\right)\right. \\
& \left.\cdot \cos (\psi(k-1)) \sin (\psi(k-1))+v_{w, N S}(k-1) v_{w, E W}(k-1) \cos ^{2}(\psi(k-1))\right) \\
& +e_{r}(k)-\theta_{1}\left(r(k-1)+e_{r}(k-1)\right) \\
& -\theta_{2}\left(u(k-1) v(k-1)+u(k-1) e_{v}(k-1)+v(k-1) e_{u}(k-1)+e_{u}(k-1) e_{v}(k-1)\right) \\
& +\theta_{3}\left(v(k-1)^{2}+2 v(k-1) e_{v}(k-1)+e_{v}(k-1)^{2}\right) \\
& +\theta_{4}\left(v(k-1) r(k-1)+v(k-1) e_{r}(k-1)+r(k-1) e_{v}(k-1)+e_{v}(k-1) e_{r}(k-1)\right) \\
& -\theta_{5} \tilde{\tau}_{\psi}(k-1)-b_{1}\left(r(k-1)+e_{r}(k-1)\right) \cos \left(\psi(k-1)+e_{\psi}(k-1)\right) \\
& -b_{2}\left(r(k-1)+e_{r}(k-1)\right) \sin \left(\psi(k-1)+e_{\psi}(k-1)\right) \\
& -b_{3}\left(v(k-1)+e_{v}(k-1)\right) \cos \left(\psi(k-1)+e_{\psi}(k-1)\right) \\
& -b_{4}\left(v(k-1)+e_{v}(k-1)\right) \sin \left(\psi(k-1)+e_{\psi}(k-1)\right) \\
& -b_{5}\left(u(k-1)+e_{u}(k-1)\right) \cos \left(\psi(k-1)+e_{\psi}(k-1)\right) \\
& -b_{6}\left(u(k-1)+e_{u}(k-1)\right) \sin \left(\psi(k-1)+e_{\psi}(k-1)\right) \\
& \left.\left.-b_{7} \cos ^{2}\left(\psi(k-1)+e_{\psi}(k-1)\right)-b_{8} \sin \left(2\left(\psi(k-1)+e_{\psi}(k-1)\right)\right)\right]\right\} \\
& =\left(1+\mathcal{N}_{r}-\theta_{1}\right) \bar{E}\left\{\zeta_{r, 1}(k) r(k-1)\right\}+\left(\mathcal{N}_{u v}+\mathcal{W}_{u v}-\theta_{2}\right) \bar{E}\left\{\zeta_{r, 1}(k) u(k-1) v(k-1)\right\}
\end{aligned}
$$




$$
\begin{aligned}
& -\left(\mathcal{N}_{|v| v}-\theta_{3}\right) \bar{E}\left\{\zeta_{r, 1}(k) v(k-1)^{2}\right\}-\left(\mathcal{N}_{|v| r}-\theta_{4}\right) \bar{E}\left\{\zeta_{r, 1}(k) v(k-1) r(k-1)\right\} \\
& +\left(\mathcal{N}_{\mu}-\theta_{5}\right) \bar{E}\left\{\zeta_{r, 1}(k) \tilde{\tau}_{\psi}(k-1)\right\} \\
& +\left(\mathcal{N}_{|v| r} \bar{v}_{c, E W}-\rho_{e_{\psi}} b_{1}\right) \bar{E}\left\{\zeta_{r, 1}(k) r(k-1) \cos (\psi(k-1))\right\} \\
& +\left(-\mathcal{N}_{|v| r} \bar{v}_{c, N S}-\rho_{e_{\psi}} b_{2}\right) \bar{E}\left\{\zeta_{r, 1}(k) r(k-1) \sin (\psi(k-1))\right\} \\
& +\left(-\mathcal{N}_{u v} \bar{v}_{c, N S}+2 \mathcal{N}_{|v| v} \bar{v}_{c, E W}-\mathcal{W}_{u v} \bar{v}_{w, N S}-\rho_{e_{\psi}} b_{3}\right) \bar{E}\left\{\zeta_{r, 1}(k) v(k-1) \cos (\psi(k-1))\right\} \\
& +\left(-\mathcal{N}_{u v} \bar{v}_{c, E W}-2 \mathcal{N}_{|v| v} \bar{v}_{c, N S}-\mathcal{W}_{u v} \bar{v}_{w, E W}-\rho_{e_{\psi}} b_{4}\right) \bar{E}\left\{\zeta_{r, 1}(k) v(k-1) \sin (\psi(k-1))\right\} \\
& +\left(-\mathcal{N}_{u v} \bar{v}_{c, E W}-\mathcal{W}_{u v} \bar{v}_{w, E W}-\rho_{e_{\psi}} b_{5}\right) \bar{E}\left\{\zeta_{r, 1}(k) u(k-1) \cos (\psi(k-1))\right\} \\
& +\left(\mathcal{N}_{u v} \bar{v}_{c, N S}+\mathcal{W}_{u v} \bar{v}_{w, N S}-\rho_{e_{\psi}} b_{6}\right) \bar{E}\left\{\zeta_{r, 1}(k) u(k-1) \sin (\psi(k-1))\right\} \\
& +\left(-\mathcal{N}_{|v| v} \bar{E}\left\{v_{c, E W}(k-1)^{2}-v_{c, N S}(k-1)^{2}\right\}+2 \mathcal{N}_{u v} \bar{E}\left\{v_{c, N S}(k-1) v_{c, E W}(k-1)\right\}\right. \\
& \left.+2 \mathcal{W}_{u v} \bar{E}\left\{v_{w, N S}(k-1) v_{w, E W}(k-1)\right\}-\left(2 \sigma_{e_{\psi}}-1\right) b_{7}\right) \bar{E}\left\{\zeta_{r, 1}(k) \cos { }^{2}(\psi(k-1))\right\} \\
& +\left(\mathcal{N}_{|v| v} \bar{E}\left\{v_{c, N S}(k-1) v_{c, E W}(k-1)\right\}+\frac{1}{2} \mathcal{N}_{u v} \bar{E}\left\{v_{c, E W}(k-1)^{2}-v_{c, N S}(k-1)^{2}\right\}\right. \\
& \left.+\frac{1}{2} \mathcal{W}_{u v} \bar{E}\left\{v_{w, E W}(k-1)^{2}-v_{w, N S}(k-1)^{2}\right\}-\left(2 \sigma_{e_{\psi}}-1\right) b_{8}\right) \bar{E}\left\{\zeta_{r, 1}(k) \sin (2 \psi(k-1))\right\}
\end{aligned}
$$

From this it can be seen that the asymptotic parameter estimates are

$$
\lim _{N \rightarrow \infty} \hat{\theta}_{N}^{I V}=\left[\begin{array}{c}
1+\mathcal{N}_{r} \\
\mathcal{N}_{u v}+\mathcal{W}_{u v} \\
\mathcal{N}_{|v| v} \\
\mathcal{N}_{|v| r} \\
\mathcal{N}_{\mu} \\
\frac{1}{\rho_{e_{\psi}}} \mathcal{N}_{|v| r} \bar{v}_{c, E W} \\
-\frac{1}{\rho_{e_{\psi}}} \mathcal{N}_{|v| r} \bar{v}_{c, N S} \\
\frac{1}{\rho_{e_{\psi}}}\left(-\mathcal{N}_{u v} \bar{v}_{c, N S}+2 \mathcal{N}_{|v| v} \bar{v}_{c, E W}-\mathcal{W}_{u v} \bar{v}_{w, N S}\right) \\
\frac{1}{\rho_{e_{\psi}}}\left(-\mathcal{N}_{u v} \bar{v}_{c, E W}-2 \mathcal{N}_{|v| v} \bar{v}_{c, N S}-\mathcal{W}_{u v} \bar{v}_{w, E W}\right) \\
-\frac{1}{\rho_{e_{\psi}}}\left(\mathcal{N}_{u v} \bar{v}_{c, E W}+\mathcal{W}_{u v} \bar{v}_{w, E W}\right) \\
\frac{1}{\rho_{e_{\psi}}}\left(\mathcal{N}_{u v} \bar{v}_{c, N S}+\mathcal{W}_{u v} \bar{v}_{w, N S}\right) \\
\frac{1}{2 \sigma_{e_{\psi}}-1} \xi_{7}\left(\nu_{c, n}, v_{w, n}\right) \\
\frac{1}{2 \sigma_{e_{\psi}}-1} \xi_{8}\left(v_{c, n}, v_{w, n}\right)
\end{array}\right]
$$


where

$$
\begin{aligned}
& \xi_{7}\left(v_{c, n}, v_{w, n}\right)=-\mathcal{N}_{|v| v}\left(\bar{E}\left\{v_{c, E W}(k-1)^{2}\right\}-\bar{E}\left\{v_{c, N S}(k-1)^{2}\right\}\right) \\
& +2\left(\mathcal{N}_{u v} \bar{E}\left\{v_{c, N S}(k-1) v_{c, E W}(k-1)\right\}+\mathcal{W}_{u v} \bar{E}\left\{v_{w, N S}(k-1) v_{w, E W}(k-1)\right\}\right) \\
& \xi_{8}\left(v_{c, n}, v_{w, n}\right)=\mathcal{N}_{|v| v} \bar{E}\left\{v_{c, N S}(k-1) v_{c, E W}(k-1)\right\} \\
& +\frac{1}{2}\left(\mathcal{N}_{u v} \bar{E}\left\{v_{c, E W}(k-1)^{2}-v_{c, N S}(k-1)^{2}\right\}+\mathcal{W}_{u v} \bar{E}\left\{v_{w, E W}(k-1)^{2}-v_{w, N S}(k-1)^{2}\right\}\right) .
\end{aligned}
$$

\section{A.5 Yaw-rate equation - with wind measurements}

Under assumption of an experiment design fulfilling (5.83), (5.82) may be rewritten as

$$
\begin{aligned}
r(k+1) & =r(k)+\mathcal{N}_{u v}\left(u(k)-u_{c}(k)\right)\left(v(k)-v_{c}(k)\right)+\mathcal{N}_{r} r(k)+\mathcal{N}_{|v| v}\left(v(k)-v_{c}(k)\right)^{2} \\
& +\mathcal{N}_{|v| r}\left(v(k)-v_{c}(k)\right) r(k)+\mathcal{W}_{u v} u_{q}(k) v_{q}(k)+\mathcal{N}_{\mu} \tilde{\tau}_{\psi}(k) \\
& =\left(1+\mathcal{N}_{r}-\mathcal{N}_{|v| r} v_{c}(k)\right) r(k)+\mathcal{N}_{u v} u(k) v(k)+\mathcal{N}_{|v| v} v(k)^{2}+\mathcal{N}_{|v| r} v(k) r(k) \\
& +\mathcal{W}_{u v} u_{q}(k) v_{q}(k)+\mathcal{N}_{\mu} \tilde{\tau}_{\psi}(k)-\left(\mathcal{N}_{u v} u_{c}(k)+2 \mathcal{N}_{|v| v} v_{c}(k)\right) v(k) \\
& -\mathcal{N}_{u v} v_{c}(k) u(k)+\mathcal{N}_{|v| v} v_{c}(k)^{2}+\mathcal{N}_{u v} u_{c}(k) v_{c}(k) .
\end{aligned}
$$

In this case, the left-hand side of the IV equation when the predictor (5.89) is used can be expressed as

$$
\begin{aligned}
& \bar{E}\left\{\zeta_{r, 2}(k)\left(y_{r}(k)-\varphi_{r, 2}^{T}(k) \theta\right)\right\}=\bar{E}\left\{\zeta _ { r , 2 } ( k ) \left[\left(1+\mathcal{N}_{r}-\mathcal{N}_{|v| r}\left(-v_{c, N S}(k-1)\right.\right.\right.\right. \\
& \left.\left.\cdot \sin (\psi(k-1))+v_{c, E W}(k-1) \cos (\psi(k-1))\right)\right) r(k-1)+\mathcal{N}_{u v} u(k-1) v(k-1) \\
& +\mathcal{N}_{|v| v} v(k-1)^{2}+\mathcal{N}_{|v| r} v(k-1) r(k-1)+\mathcal{W}_{u v} u_{q}(k-1) v_{q}(k-1)+\mathcal{N}_{\mu} \tilde{\tau}_{\psi}(k-1) \\
& -\left(\mathcal{N}_{u v}\left(v_{c, N S}(k-1) \cos (\psi(k-1))+v_{c, E W}(k-1) \sin (\psi(k-1))\right)+2 \mathcal{N}_{|v| v}\left(-v_{c, N S}(k-1)\right.\right. \\
& \left.\left.\cdot \sin (\psi(k-1))+v_{c, E W}(k-1) \cos (\psi(k-1))\right)\right) v(k-1)-\mathcal{N}_{u v}\left(-v_{c, N S}(k-1)\right. \\
& \left.\cdot \sin (\psi(k-1))+v_{c, E W}(k-1) \cos (\psi(k-1))\right) u(k-1)+\mathcal{N}_{|v| v}\left(v_{c, N S}(k-1)^{2} \sin ^{2}(\psi(k-1))\right. \\
& \left.-2 v_{c, N S}(k-1) v_{c, E W}(k-1) \cos (\psi(k-1)) \sin (\psi(k-1))+v_{c, E W}(k-1)^{2} \cos ^{2}(\psi(k-1))\right) \\
& +\mathcal{N}_{u v}\left(-v_{c, N S}(k-1) v_{c, E W}(k-1) \sin ^{2}(\psi(k-1))-\left(v_{c, N S}(k-1)^{2}-v_{c, E W}(k-1)^{2}\right)\right.
\end{aligned}
$$




$$
\begin{aligned}
& \left.\cdot \cos (\psi(k-1)) \sin (\psi(k-1))+v_{c, N S}(k-1) v_{c, E W}(k-1) \cos ^{2}(\psi(k-1))\right) \\
& +e_{r}(k)-\theta_{1}\left(r(k-1)+e_{r}(k-1)\right) \\
& -\theta_{2}\left(u(k-1) v(k-1)+u(k-1) e_{v}(k-1)+v(k-1) e_{u}(k-1)+e_{u}(k-1) e_{v}(k-1)\right) \\
& -\theta_{3}\left(v(k-1)^{2}+2 v(k-1) e_{v}(k-1)+e_{v}(k-1)^{2}\right) \\
& -\theta_{4}\left(v(k-1) r(k-1)+v(k-1) e_{r}(k-1)+r(k-1) e_{v}(k-1)+e_{v}(k-1) e_{r}(k-1)\right) \\
& -\theta_{5}\left(u_{q}(k-1) v_{q}(k-1)+u_{q}(k-1) e_{v_{q}}(k-1)+v_{q}(k-1) e_{u_{q}}(k-1)+e_{u_{q}}(k-1) e_{v_{q}}(k-1)\right) \\
& -\theta_{6} \tilde{\tau}_{\psi}(k-1)-b_{1}\left(r(k-1)+e_{r}(k-1)\right) \cos \left(\psi(k-1)+e_{\psi}(k-1)\right) \\
& -b_{2}\left(r(k-1)+e_{r}(k-1)\right) \sin \left(\psi(k-1)+e_{\psi}(k-1)\right) \\
& -v_{3}\left(v(k-1)+e_{v}(k-1)\right) \cos \left(\psi(k-1)+e_{\psi}(k-1)\right) \\
& -b_{4}\left(v(k-1)+e_{v}(k-1)\right) \sin \left(\psi(k-1)+e_{\psi}(k-1)\right) \\
& -b_{5}\left(u(k-1)+e_{u}(k-1)\right) \cos \left(\psi(k-1)+e_{\psi}(k-1)\right) \\
& -b_{6}\left(u(k-1)+e_{u}(k-1)\right) \sin \left(\psi(k-1)+e_{\psi}(k-1)\right) \\
& \left.\left.-b_{7} \cos ^{2}\left(\psi(k-1)+e_{\psi}(k-1)\right)-b_{8} \sin \left(2\left(\psi(k-1)+e_{\psi}(k-1)\right)\right)\right]\right\} \\
& =\left(1+\mathcal{N}_{r}-\theta_{1}\right) \bar{E}\left\{\zeta_{r, 2}(k) r(k-1)\right\}+\left(\mathcal{N}_{u v}-\theta_{2}\right) \bar{E}\left\{\zeta_{r, 2}(k) u(k-1) v(k-1)\right\} \\
& +\left(\mathcal{N}_{|v| v}-\theta_{3}\right) \bar{E}\left\{\zeta_{r, 2}(k) v(k-1)^{2}\right\}+\left(\mathcal{N}_{|v| r}-\theta_{4}\right) \bar{E}\left\{\zeta_{r, 2}(k) v(k-1) r(k-1)\right\} \\
& +\left(\mathcal{W}_{u v}-\theta_{5}\right) \bar{E}\left\{\zeta_{r, 2}(k) u_{q}(k-1) v_{q}(k-1)\right\}+\left(\mathcal{N}_{\mu}-\theta_{6}\right) \bar{E}\left\{\zeta_{r, 2}(k) \tilde{\tau}_{\psi}(k-1)\right\} \\
& +\left(-\mathcal{N}_{|v| r} \bar{v}_{c, E W}-\rho_{e_{\psi}} b_{1}\right) \bar{E}\left\{\zeta_{r, 2}(k) r(k-1) \cos (\psi(k-1))\right\} \\
& +\left(\mathcal{N}_{|v| r} \bar{v}_{c, N S}-\rho_{e_{\psi}} b_{2}\right) \bar{E}\left\{\zeta_{r, 2}(k) r(k-1) \sin (\psi(k-1))\right\} \\
& +\left(-\mathcal{N}_{u v} \bar{v}_{c, N S}-2 \mathcal{N}_{|v| v} \bar{v}_{c, E W}-\rho_{e_{\psi}} b_{3}\right) \bar{E}\left\{\zeta_{r, 2}(k) v(k-1) \cos (\psi(k-1))\right\} \\
& +\left(-\mathcal{N}_{u v} \bar{v}_{c, E W}+2 \mathcal{N}_{|v| v} \bar{v}_{c, N S}-\rho_{e_{\psi}} b_{4}\right) \bar{E}\left\{\zeta_{r, 2}(k) v(k-1) \sin (\psi(k-1))\right\} \\
& +\left(-\mathcal{N}_{u v} \bar{v}_{c, E W}-\rho_{e_{\psi}} b_{5}\right) \bar{E}\left\{\zeta_{r, 2}(k) u(k-1) \cos (\psi(k-1))\right\} \\
& +\left(\mathcal{N}_{u v} \bar{v}_{c, N S}-\rho_{e_{\psi}} b_{6}\right) \bar{E}\left\{\zeta_{r, 2}(k) u(k-1) \sin (\psi(k-1))\right\} \\
& +\left(\mathcal{N}_{|v| v} \bar{E}\left\{v_{c, E W}(k-1)^{2}-v_{c, N S}(k-1)^{2}\right\}+2 \mathcal{N}_{u v} \bar{E}\left\{v_{c, N S}(k-1) v_{c, E W}(k-1)\right\}\right. \\
& \left.-\left(2 \sigma_{e_{\psi}}-1\right) b_{7}\right) \bar{E}\left\{\zeta_{r, 2}(k) \cos ^{2}(\psi(k-1))\right\} \\
& +\left(-\mathcal{N}_{|v| v} \bar{E}\left\{v_{c, N S}(k-1) v_{c, E W}(k-1)\right\}+\frac{1}{2} \mathcal{N}_{u v} \bar{E}\left\{v_{w, E W}(k-1)^{2}-v_{w, N S}(k-1)^{2}\right\}\right.
\end{aligned}
$$




$$
\left.-\left(2 \sigma_{e_{\psi}}-1\right) b_{8}\right) \bar{E}\left\{\zeta_{r, 2}(k) \sin (2 \psi(k-1))\right\} \text {. }
$$

From this it can be seen that the asymptotic parameter estimates

$$
\lim _{N \rightarrow \infty} \hat{\theta}_{N}^{I V}=\left[\begin{array}{c}
1+\mathcal{N}_{r} \\
\mathcal{N}_{u v} \\
\mathcal{N}_{|v| v} \\
\mathcal{N}_{|v| r} \\
\mathcal{W}_{u v} \\
\mathcal{N}_{\mu} \\
-\frac{1}{\rho_{e_{\psi}}} \mathcal{N}_{|v| r} \bar{v}_{c, E W} \\
\frac{1}{\rho_{e_{\psi}}} \mathcal{N}_{|v| r} \bar{v}_{c, N S} \\
\frac{1}{\rho_{e_{\psi}}}\left(-\mathcal{N}_{u v} \bar{v}_{c, N S}-2 \mathcal{N}_{|v| v} \bar{v}_{c, E W}\right) \\
\frac{1}{\rho_{e_{\psi}}}\left(-\mathcal{N}_{u v} \bar{v}_{c, E W}+2 \mathcal{N}_{|v| v} \bar{v}_{c, N S}\right) \\
-\frac{1}{\rho_{e_{\psi}}} \mathcal{N}_{u v} \bar{v}_{c, E W} \\
\frac{1}{\rho_{e_{\psi}}} \mathcal{N}_{u v} \bar{v}_{c, N S} \\
\frac{1}{2 \sigma_{e_{\psi}}-1} \xi_{5}\left(v_{c, n}, 0\right) \\
\frac{1}{2 \sigma_{e_{\psi}}-1} \xi_{6}\left(v_{c, n}, 0\right)
\end{array}\right] \text {, }
$$

follow. Here, $\xi_{5}($.$) and \xi_{6}($.$) are defined by (5.100) and (5.101), respectively. If$ the experiments are done such that (5.84) is fulfilled, the expressions for the estimated disturbance terms change in a way that is similar to the case without wind measurements handled above. However, the parameters needed for performing undisturbed simulations stay the same. 



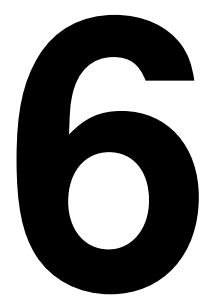

\section{Simulation Study}

In order to illustrate the potential of the estimators derived throughout Chapters 4 and 5, simulation studies have been performed. The results of these studies are presented in this chapter. First, the general simulation setup is described. After that, the results of two sets of Monte Carlo simulations are presented. These simulations show the convergences proclaimed in Chapter 5. Finally, the results of a set of simulations are shown, where the amount of estimation data is varied.

\subsection{Simulation setup}

Throughout all simulations, data was generated based on the maneuvering model

$$
\begin{aligned}
{\left[\begin{array}{l}
u(k) \\
v(k) \\
r(k)
\end{array}\right] } & =\left[\begin{array}{l}
u(k-1) \\
v(k-1) \\
r(k-1)
\end{array}\right]+\left[\begin{array}{ccc}
\varphi_{u, 0}^{T}(k) & 0 & 0 \\
0 & \varphi_{v, 0}^{T}(k) & 0 \\
0 & 0 & \varphi_{r, 0}^{T}(k)
\end{array}\right] \theta_{0}, \\
\mathbf{y}(k) & =\left[\begin{array}{l}
y_{u}(k) \\
y_{v}(k) \\
y_{r}(k) \\
y_{\psi}(k)
\end{array}\right]=\left[\begin{array}{c}
u(k) \\
v(k) \\
r(k) \\
\psi(k)
\end{array}\right]+\left[\begin{array}{c}
e_{u}(k) \\
e_{v}(k) \\
e_{r}(k) \\
e_{\psi}(k)
\end{array}\right]
\end{aligned}
$$

defined by (3.51), with unit sampling time and $\theta_{0}$ according to the middle column of Table 6.1. This $\theta_{0}$ was chosen based on the models found using the experimental data presented in Chapter 7 , but the aerodynamic coefficients were scaled up slightly. Conceivably, this could correspond to a case where the ship is loaded differently and therefore has a bigger area exposed to the surrounding air. 
Table 6.1: System premises for simulation.

\begin{tabular}{||c|c|c||}
\hline Parameter & True system & Nominal model \\
\hline $\mathcal{X}_{u}$ & -0.05 & -0.2 \\
\hline $\mathcal{X}_{v r}$ & 1 & 0.8 \\
\hline $\mathcal{X}_{|u| u}$ & -0.05 & 0 \\
\hline $\mathcal{W}_{|u| u}$ & -0.0005 & 0 \\
\hline $\mathcal{X}_{\mu}$ & 0.02 & 0.01 \\
\hline $\mathcal{X}_{\kappa}$ & -0.0025 & 0 \\
\hline $\mathcal{Y}_{v}$ & -0.2 & -0.3 \\
\hline $\mathcal{Y}_{u r}$ & -0.65 & -0.8 \\
\hline $\mathcal{Y}_{|v| v}$ & -0.2 & 0 \\
\hline $\mathcal{Y}_{|v| r}$ & -0.1 & 0 \\
\hline $\mathcal{W}_{|v| v}$ & -0.0015 & 0 \\
\hline $\mathcal{Y}_{\mu}$ & 0.02 & 0.01 \\
\hline $\mathcal{N}_{r}$ & -0.1 & -0.15 \\
\hline $\mathcal{N}_{u v}$ & -0.0015 & 0 \\
\hline $\mathcal{N}_{|v| v}$ & -0.001 & 0 \\
\hline $\mathcal{N}_{|v| r}$ & -0.04 & 0 \\
\hline $\mathcal{W}_{u v}$ & -0.00003 & 0 \\
\hline $\mathcal{N}_{\mu}$ & 0.0003 & 0.00015 \\
\hline
\end{tabular}

All disturbances were sampled from Gaussian distributions

$$
\begin{aligned}
e_{u}(k) & \sim \mathcal{N}\left(0,2 \cdot 10^{-4}\right), \\
e_{v}(k) & \sim \mathcal{N}\left(0,2 \cdot 10^{-4}\right), \\
e_{r}(k) & \sim \mathcal{N}\left(0,2 \cdot 10^{-4}\right), \\
e_{\psi}(k) & \sim \mathcal{N}\left(0,10^{-4}\right), \\
v_{c, N S}(k) & \sim \mathcal{N}\left(0.2,10^{-3}\right), \\
v_{c, E W}(k) & \sim \mathcal{N}\left(-0.2,10^{-3}\right), \\
v_{w, N S}(k) & \sim \mathcal{N}\left(\bar{v}_{w, N S}, 10^{-3}\right), \\
v_{w, E W}(k) & \sim \mathcal{N}\left(\bar{v}_{w, E W}, 10^{-3}\right),
\end{aligned}
$$

which means that neither the distributions of the measurement noises nor of the environmental disturbances did have finite support.

Six estimators were compared in the simulation study, three LS estimators denoted $\hat{\theta}_{N}^{L S_{1}}, \hat{\theta}_{N}^{L S_{2}}, \hat{\theta}_{N}^{L S_{3}}$, and three IV estimators denoted $\hat{\theta}_{N}^{I V_{1}}, \hat{\theta}_{N}^{I V_{2}}, \hat{\theta}_{N}^{I V_{3}}$. The 
estimators $\hat{\theta}_{N}^{L S_{1}}$ and $\hat{\theta}_{N}^{I V_{1}}$ relied on basic predictors in each DOF

$$
\begin{aligned}
& {\left[\begin{array}{c}
\hat{y}_{u, 6}(k \mid \theta) \\
\hat{y}_{v, 3}(k \mid \theta) \\
\hat{y}_{r, 3}(k \mid \theta)
\end{array}\right]=\left[\begin{array}{ccc}
\varphi_{u, 6}^{T}(k) & 0 & 0 \\
0 & \varphi_{v, 3}^{T}(k) & 0 \\
0 & 0 & \varphi_{r, 3}^{T}(k)
\end{array}\right]\left[\begin{array}{c}
\theta_{1} \\
\vdots \\
\theta_{15}
\end{array}\right],} \\
& \varphi_{u, 6}(k)=\left[y_{u}(k-1) \quad y_{v}(k-1) y_{r}(k-1) \quad y_{u}(k-1)\left|y_{u}(k-1)\right| \ldots\right. \\
& \left.\ldots \quad \tilde{\tau}_{x}(k-1) \quad y_{u}(k-1) \tilde{\tau}_{x}(k-1)\right]^{T} \text {, } \\
& \varphi_{v, 3}(k)=\left[y_{v}(k-1) \quad y_{u}(k-1) y_{r}(k-1) \quad y_{v}(k-1)\left|y_{v}(k-1)\right| \ldots\right. \\
& \left.\ldots \quad y_{r}(k-1)\left|y_{v}(k-1)\right| \quad \tilde{\tau}_{y}(k-1)\right]^{T} \text {, } \\
& \varphi_{r, 3}(k)=\left[\begin{array}{lll}
y_{r}(k-1) & y_{u}(k-1) y_{v}(k-1) & y_{v}(k-1)\left|y_{v}(k-1)\right|
\end{array} \ldots\right. \\
& \left.\ldots y_{r}(k-1)\left|y_{v}(k-1)\right| \quad \tilde{\tau}_{\psi}(k-1)\right]^{T} \text {. }
\end{aligned}
$$

The other estimators used the augmented predictors suggested in Chapter 5. Two of them, $\hat{\theta}_{N}^{L S_{2}}$ and $\hat{\theta}_{N}^{I V_{2}}$, did not utilize wind measurements and were based on the predictor model

$$
\left[\begin{array}{c}
\hat{y}_{u, 4}(k \mid \theta) \\
\hat{y}_{v, 1}(k \mid \theta) \\
\hat{y}_{r, 1}(k \mid \theta)
\end{array}\right]=\left[\begin{array}{ccc}
\varphi_{u, 4}^{T}(k) & 0 & 0 \\
0 & \varphi_{v, 1}^{T}(k) & 0 \\
0 & 0 & \varphi_{r, 1}^{T}(k)
\end{array}\right] \theta,
$$

where $\varphi_{u, 4}^{T}(k), \varphi_{v, 1}^{T}(k)$ and $\varphi_{r, 1}^{T}(k)$ are given by (5.61), (5.70) and (5.85), respectively. Here the parameter vector is partitioned as

$$
\theta=\left[\begin{array}{lllllll}
\theta_{1} \ldots \theta_{5} & b_{1} \ldots b_{10} & \theta_{6} \ldots \theta_{10} & b_{11} \ldots b_{18} & \theta_{11} \ldots \theta_{15} & b_{19} \ldots b_{26}
\end{array}\right]^{T},
$$

such that $\theta_{1} \ldots \theta_{15}$ completely define the undisturbed simulation model. The final two estimators, $\hat{\theta}_{N}^{L S_{3}}$ and $\hat{\theta}_{N}^{I V_{3}}$, also utilized wind measurements

$$
\left[\begin{array}{c}
\hat{y}_{u, 5}(k \mid \theta) \\
\hat{y}_{v, 2}(k \mid \theta) \\
\hat{y}_{r, 2}(k \mid \theta)
\end{array}\right]=\left[\begin{array}{ccc}
\varphi_{u, 5}^{T}(k) & 0 & 0 \\
0 & \varphi_{v, 2}^{T}(k) & 0 \\
0 & 0 & \varphi_{r, 2}^{T}(k)
\end{array}\right] \theta .
$$

Here $\varphi_{u, 5}^{T}(k), \varphi_{v, 2}^{T}(k)$ and $\varphi_{r, 2}^{T}(k)$ are given by (5.64), (5.75) and (5.88), respectively. In this case, the parameter vector is partitioned as

$$
\theta=\left[\begin{array}{lllllll}
\theta_{1} \ldots \theta_{6} & b_{1} \ldots b_{10} & \theta_{7} \ldots \theta_{12} & b_{11} \ldots b_{18} & \theta_{13} \ldots \theta_{18} & b_{19} \ldots b_{26}
\end{array}\right]^{T} .
$$


Notably, these estimators provide more information by estimating the aerodynamic drag coefficients independently.

As in the simulations carried out in Chapter 4, a refinement procedure was performed for the IV estimators. Here, the first set of instruments were however obtained by simulation of a nominal model with crude parameter values. The nominal model had the same structure as the true system and its parameters are given in the right column of Table 6.1. All the IV estimators used zero-mean instruments. In order to obtain zero-mean instruments, the average value of each component of the instrument vector was simply subtracted.

The actuator configuration was adopted from the ship studied in Chapter 7 . That ship has two azimuth thrusters mounted along the centerline, one at the front and one in the rear. The input was generated by rotating both these thrusters with a nominal offset $\bar{\alpha}=\pi / 4$ radians and having both propellers run with the same positive nominal speed $\bar{n}=10$. The propellers' speeds and angles were then varied around these nominal values

$$
\begin{aligned}
& n_{1}(k)=n_{2}(k)=\bar{n}+\tilde{n}(k), \\
& \alpha_{1}(k)=\bar{\alpha}+\tilde{\alpha}_{1}(k), \\
& \alpha_{2}(k)=\bar{\alpha}+\tilde{\alpha}_{2}(k) .
\end{aligned}
$$

The time-varying components $\tilde{n}(k), \tilde{\alpha}_{1}(k)$ and $\tilde{\alpha}_{2}(k)$ were smoothed pulses of varying width that excited the system well. With an input signal like this the ship has a positive surge and sway speed. The data therefore fulfills the requirements of having the surge and sway states well-separated from the origin, simultaneously.

\subsection{Convergence}

In order to illustrate the consistency results found in Chapter 5, histograms of estimation errors following Monte Carlo simulations would ideally have been shown. Since there are many unknown parameters to estimate in the maneuvering model (3.51), this would however have required a large number of histograms. It was therefore decided to instead show the results in a table format, providing the estimation errors as an average plus/minus one standard deviation for each parameter. Here, the property of the origin being within this spun interval will be taken as an indication of consistency. It can however be noted that an estimation bias can be obscured by a high variance and also that a seemingly significant bias might vanish when $N \rightarrow \infty$. It is consequently hard to prove the consistency of an estimator using data.

To begin with, a set of 500 simulations were performed with moderately low wind velocity, $\bar{v}_{w, N S}=\bar{v}_{w, E W}=1 \mathrm{~m} / \mathrm{s}$. In each Monte Carlo iteration, $N=5000$ data points were used for parameter estimation. Data from one of these experiments is shown in Figure 6.1. It can be noted that the ship for the major part of the experiment is moving faster than the surrounding air. This means that the conditions (5.7), (5.32), (5.68) and (5.69), for the most part are fulfilled. Estimation 

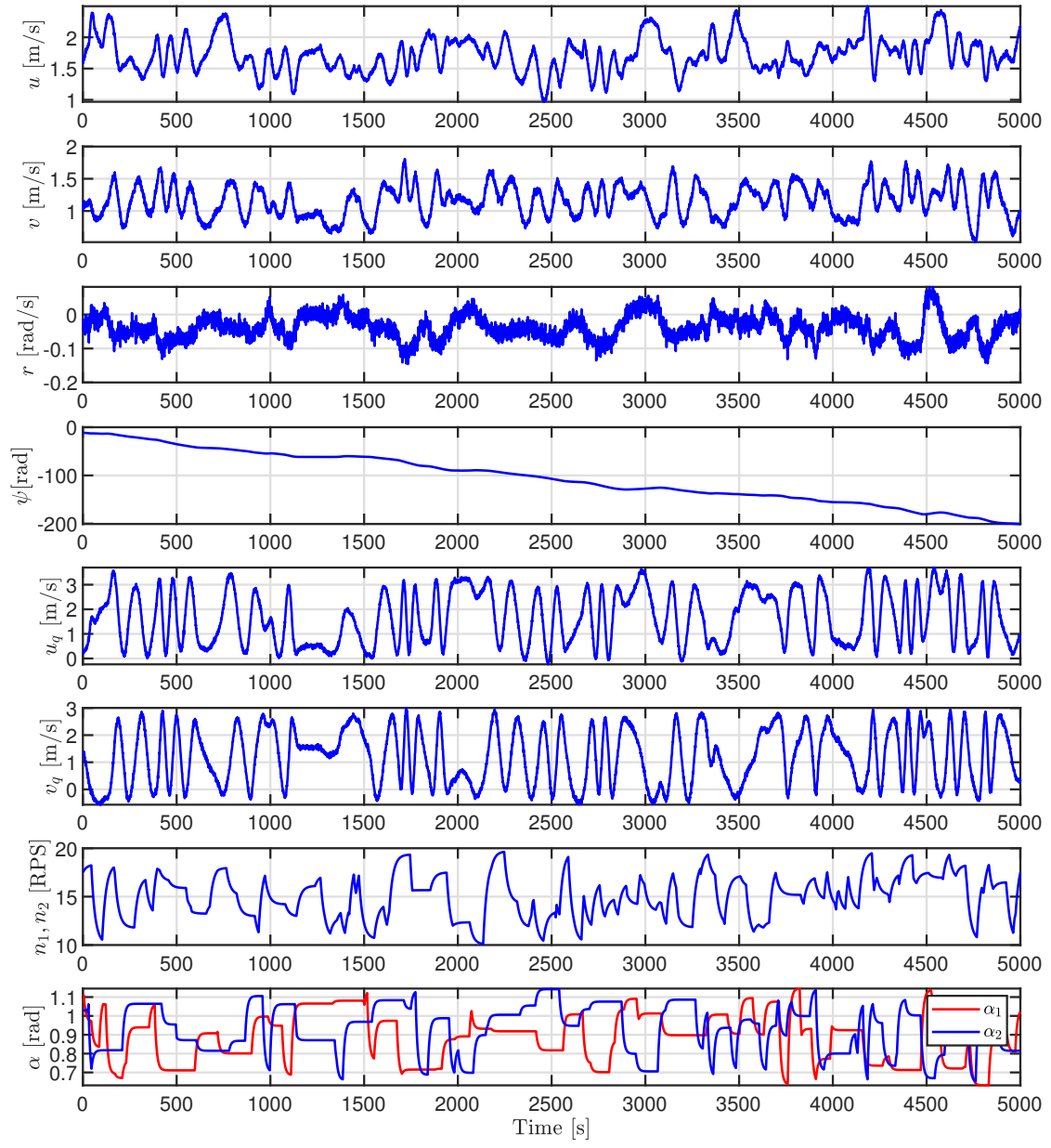

Figure 6.1: Example of a set of simulated data from the Monte Carlo simulations with low wind speed, $\bar{v}_{w, N S}=\bar{v}_{w, E W}=1 \mathrm{~m} / \mathrm{s}$.

errors for the six estimators are provided in Tables 6.2, 6.3 and 6.4. In Tables 6.2 and 6.4, it can be seen that neither of the LS estimators seem consistent. Actually, the only two estimators that seem consistent in this scenario are $\hat{\theta}_{N}^{I V_{2}}$ and $\hat{\theta}_{N}^{I V_{3}}$ because as seen in Table 6.3, also the IV estimator using basic predictors in each DOF seems inconsistent. From the estimators $\hat{\theta}_{N}^{L S_{3}}$ and $\hat{\theta}_{N}^{I V_{3}}$, independent estimates of the aerodynamic drag coefficients are obtained. Notably, these estimates are quite uncertain which is reasonable following the mild wind conditions. The high standard deviation also partly depends on the way the estimation errors are normalized.

After this, another set of 500 simulations were performed. This time a higher wind speed, $\bar{v}_{w, N S}=\bar{v}_{w, E W}=10 \mathrm{~m} / \mathrm{s}$, was used. Estimation errors for the six 
estimators are this time provided in Tables 6.5, 6.6 and 6.7. In this case, only the IV estimator that utilizes wind measurements seems to be consistent. The fact that $\hat{\theta}_{N}^{I V_{2}}$ is biased in this scenario is reasonable, because in this simulation setup the wind moves much faster than the ship. Therefore, the conditions (5.7), (5.32), (5.68) and (5.69) are occasionally violated during the simulation experiments, which results in biased estimates of the parameters associated with the surge and sway predictors. Recall that for the yaw-rate predictor, the milder experiment conditions (5.83) and (5.84) could be considered. These conditions do not depend on the wind and in Table 6.6, it can be seen that the parameters associated with the yaw-rate predictor are still estimated accurately by $\hat{\theta}_{N}^{I V_{2}}$, despite the increased wind speed. For the estimators utilizing wind measurements, the estimates of the aerodynamic drag coefficients associated with surge and yaw rate can in Table 6.7 be seen to be more accurate than in Table 6.4. This is likely due to the increased aerodynamic excitation that follows the more severe wind conditions in the second set of simulations.

\subsection{Model fit}

In a situation with limited amount of estimation data, an inconsistent estimator might very well give better performing models than a consistent one. In order to evaluate the variance properties of the suggested estimators, some simulations were therefore performed where the amount of estimation data was varied. Instead of looking at parameter-estimation errors, the results of these simulations were assessed using the normalized model-fit metric

$$
\text { fit }(\hat{y}(k \mid \theta))=100\left(1-\sqrt{\frac{\sum_{k=1}^{N}(y(k)-\hat{y}(k))^{2}}{\sum_{k=1}^{N}\left(y(k)-\frac{1}{N} \sum_{k=1}^{N} y(k)\right)^{2}}}\right) .
$$

This metric is sometimes interpreted as a percentage because the best possible outcome is 100. The output of a model can however have arbitrarily bad fit and values below 0 are therefore also possible.

Also these simulations were divided into the two cases with moderately low wind speed, $\bar{v}_{w, N S}=\bar{v}_{w, E W}=1 \mathrm{~m} / \mathrm{s}$, and high wind speed, $\bar{v}_{w, N S}=\bar{v}_{w, E W}=10 \mathrm{~m} / \mathrm{s}$. The model fit was calculated by comparing the simulated response with an undisturbed set of validation data. In the validation dataset, a more conventional input signal was used, which made the ship move forward in a zig-zag manner. The more conventional input signal was used in order to evaluate the models in a more realistic scenario. The results are given in Figures 6.2 and 6.3, for the two cases respectively. The figures were obtained by averaging the results of 100 Monte Carlo iterations, for different values of $N$ between 1000 and 5000. The average values of fit plus/minus one standard deviation are marked with triangles. In Figure 6.2, it can be seen that for low amounts of data, the models obtained from $\hat{\theta}_{N}^{L S_{2}}$ and $\hat{\theta}_{N}^{L S_{3}}$ are performing better than models found from their corre- 
sponding IV estimators. However, after a certain breakpoint the accuracy of the models obtained with the estimators $\hat{\theta}_{N}^{I V_{2}}$ and $\hat{\theta}_{N}^{I V_{3}}$, seem to overtake the accuracy of those obtained with LS estimators. In the case of high wind speed, the shortcomings of the estimator $\hat{\theta}_{N}^{I V_{2}}$ are clear, especially in surge. The corresponding LS estimator, $\hat{\theta}_{N}^{L S_{2}}$, also seems to give less accurate models in this scenario.

The regular estimators, $\hat{\theta}_{N}^{L S_{1}}$ and $\hat{\theta}_{N}^{I V_{1}}$, generate models with low model fit in both cases and in order to make the other results clear, the figures are retained to only show values of model fit between 0 and 100. As a consequence, the results of those estimators are sometimes not shown. 
Table 6.2: The mean plus/minus one standard deviation of normalized estimation errors for two LS estimators not utilizing wind measurements. Data from the set of Monte Carlo runs with low wind speed was used for estimation.

\begin{tabular}{|c|c|c|}
\hline & $L S_{1}$ & $L S_{2}$ \\
\hline$\frac{\hat{\theta}_{1}-\mathcal{X}_{u}}{\left|\mathcal{X}_{u}\right|}$ & $0.0401 \pm 0.0010$ & $0.0398 \pm 0.0039$ \\
\hline$\frac{\hat{\theta}_{2}-\mathcal{X}_{r v}}{\left|\mathcal{X}_{r v}\right|}$ & $-0.9107 \pm 0.0042$ & $-0.6689 \pm 0.0158$ \\
\hline$\frac{\hat{\theta}_{3}-\left(\mathcal{X}_{|u| u}+\mathcal{W}_{|u| u}\right)}{\left|\mathcal{X}_{|u| u}+\mathcal{W}_{|u| u}\right|}$ & $1.0345 \pm 0.0100$ & $0.4916 \pm 0.0403$ \\
\hline$\frac{\hat{\theta}_{4}-\mathcal{X}_{\mu}}{\left|\mathcal{X}_{\mu}\right|}$ & $-0.9030 \pm 0.0049$ & $-0.6815 \pm 0.0222$ \\
\hline$\frac{\hat{\theta}_{5}-\mathcal{X}_{\kappa}}{\left|\mathcal{X}_{\kappa}\right|}$ & $0.7986 \pm 0.0192$ & $0.9269 \pm 0.0701$ \\
\hline$\frac{\hat{\theta}_{6}-\mathcal{Y}_{v}}{\left|\mathcal{Y}_{v}\right|}$ & $0.2320 \pm 0.0007$ & $0.0719 \pm 0.0056$ \\
\hline$\frac{\hat{\theta}_{7}-\mathcal{Y}_{u r}}{\left|\mathcal{Y}_{u r}\right|}$ & $0.4403 \pm 0.0186$ & $0.5888 \pm 0.0379$ \\
\hline$\frac{\hat{\theta}_{8}-\left(\mathcal{Y}_{|v| v}-\mathcal{W}_{|v| v}\right)}{\left|\mathcal{Y}_{|v| v}-\mathcal{W}_{|v| v}\right|}$ & $1.0262 \pm 0.0022$ & $0.3519 \pm 0.0163$ \\
\hline$\frac{\hat{\theta}_{9}-\mathcal{Y}_{|v| r}}{\left|\mathcal{Y}_{|v| r}\right|}$ & $5.6544 \pm 0.1574$ & $-0.7297 \pm 0.3399$ \\
\hline$\frac{\hat{\theta}_{10}-\mathcal{Y}_{\mu}}{\left|\mathcal{Y}_{\mu}\right|}$ & $-0.9817 \pm 0.0008$ & $-0.3264 \pm 0.0163$ \\
\hline$\frac{\hat{\theta}_{11}-\mathcal{N}_{r}}{\left|\mathcal{N}_{r}\right|}$ & $-0.3801 \pm 0.0239$ & $-0.5614 \pm 0.0394$ \\
\hline$\frac{\hat{\theta}_{12}-\left(\mathcal{N}_{u v}+\mathcal{W}_{u v}\right)}{\left|\mathcal{N}_{u v}+\mathcal{W}_{u v}\right|}$ & $-3.9929 \pm 0.2840$ & $-3.5999 \pm 0.4637$ \\
\hline$\frac{\hat{\theta}_{13}-\mathcal{N}_{|v| v}}{\left|\mathcal{N}_{|v| v \mid}\right|}$ & $-3.7116 \pm 0.6099$ & $-8.1639 \pm 1.0556$ \\
\hline$\frac{\hat{\theta}_{14}-\mathcal{N}_{|v| r}}{\left|\mathcal{N}_{|v| r \mid}\right|}$ & $-5.4772 \pm 0.4391$ & $-4.3743 \pm 0.7357$ \\
\hline$\frac{\hat{\theta}_{15}-\mathcal{N}_{\mu}}{\left|\mathcal{N}_{\mu}\right|}$ & $4.0316 \pm 0.1033$ & $4.5444 \pm 0.1145$ \\
\hline
\end{tabular}


Table 6.3: The mean plus/minus one standard deviation of normalized estimation errors for two IV estimators not utilizing wind measurements. Data from the set of Monte Carlo runs with low wind speed was used for estimation.

\begin{tabular}{||c|c|c||}
\hline & $I V_{1}$ & $I V_{2}$ \\
\hline$\frac{\hat{\theta}_{1}-\mathcal{X}_{u}}{\left|\mathcal{X}_{u}\right|}$ & $1.8895 \pm 0.2385$ & $-0.0008 \pm 0.0289$ \\
\hline$\frac{\hat{\theta}_{2}-\mathcal{X}_{r v}}{\left|\mathcal{X}_{r v}\right|}$ & $1.3503 \pm 0.2421$ & $0.0013 \pm 0.0374$ \\
\hline$\frac{\hat{\theta}_{3}-\left(\mathcal{X}_{|u| u}+\mathcal{W}_{|u| u}\right)}{\left|\mathcal{X}_{|u| u}+\mathcal{W}_{|u| u}\right|}$ & $-15.1362 \pm 1.9721$ & $0.0037 \pm 0.2056$ \\
\hline$\frac{\hat{\theta}_{4}-\mathcal{X}_{\mu}}{\left|\mathcal{X}_{\mu}\right|}$ & $-1.4233 \pm 0.1925$ & $0.0021 \pm 0.0640$ \\
\hline$\frac{\hat{\theta}_{5}-\mathcal{X}_{\kappa}}{\left|\mathcal{X}_{k}\right|}$ & $10.9371 \pm 1.3990$ & $-0.0061 \pm 0.2313$ \\
\hline$\frac{\hat{\theta}_{6}-\mathcal{Y}_{v}}{\left|\mathcal{Y}_{v}\right|}$ & $0.7552 \pm 0.0836$ & $-0.0072 \pm 0.0679$ \\
\hline$\frac{\hat{\theta}_{7}-\mathcal{Y}_{u r}}{\left|\mathcal{Y}_{u r}\right|}$ & $0.2307 \pm 0.0602$ & $-0.0125 \pm 0.1104$ \\
\hline$\frac{\hat{\theta}_{8}-\left(\mathcal{Y}_{|v| v}-\mathcal{W}_{|v| v}\right)}{\left|\mathcal{Y}_{|v| v}-\mathcal{Y}_{|v| v}\right|}$ & $-0.5611 \pm 0.1489$ & $-0.0086 \pm 0.1304$ \\
\hline$\frac{\hat{\theta}_{9}-\mathcal{Y}_{|v| r}}{\left|\mathcal{Y}_{|v| r}\right|}$ & $2.8439 \pm 0.4505$ & $-0.0330 \pm 0.6008$ \\
\hline$\frac{\hat{\theta}_{10}-\mathcal{Y}_{\mu}}{\left|\mathcal{Y}_{\mu}\right|}$ & $-0.5728 \pm 0.0226$ & $0.0139 \pm 0.0861$ \\
\hline$\frac{\hat{\theta}_{11}-\mathcal{N}_{r}}{\left|\mathcal{N}_{r}\right|}$ & $0.0302 \pm 0.0121$ & $-0.0016 \pm 0.0111$ \\
\hline$\frac{\hat{\theta}_{12}-\left(\mathcal{N}_{u v}+\mathcal{W}_{u v}\right)}{\left|\mathcal{N}_{u v}+\mathcal{W}_{u v}\right|}$ & $-0.0833 \pm 0.1217$ & $-0.0064 \pm 0.1047$ \\
\hline$\frac{\hat{\theta}_{13}-\mathcal{N}_{|v| v}}{\left|\mathcal{N}_{|v| v}\right|}$ & $-0.4563 \pm 0.3229$ & $-0.0163 \pm 0.2068$ \\
\hline$\frac{\hat{\theta}_{14}-\mathcal{N}_{|v| r}}{\left|\mathcal{N}_{|v| r}\right|}$ & $-0.5657 \pm 0.2404$ & $-0.0026 \pm 0.1708$ \\
\hline$\frac{\hat{\theta}_{15}-\mathcal{N}_{\mu}}{\left|\mathcal{N}_{\mu}\right|}$ & $0.0090 \pm 0.0698$ & $0.0096 \pm 0.0480$ \\
\hline
\end{tabular}


Table 6.4: The mean plus/minus one standard deviation of normalized estimation errors for two estimators utilizing wind measurements. Data from the set of Monte Carlo runs with low wind speed was used for estimation.

\begin{tabular}{||c|c|c||}
\hline & $L S_{3}$ & $I V_{3}$ \\
\hline$\frac{\hat{\theta}_{1}-\mathcal{X}_{u}}{\left|\mathcal{X}_{u}\right|}$ & $0.0157 \pm 0.0047$ & $-0.0007 \pm 0.0289$ \\
\hline$\frac{\hat{\theta}_{2}-\mathcal{X}_{r v}}{\left|\mathcal{X}_{r v}\right|}$ & $-0.6770 \pm 0.0158$ & $0.0013 \pm 0.0374$ \\
\hline$\frac{\hat{\theta}_{3}-\mathcal{X}_{|u| u}}{\left|\mathcal{X}_{|u| u \mid}\right|}$ & $0.3411 \pm 0.0457$ & $-0.0013 \pm 0.2119$ \\
\hline$\frac{\hat{\theta}_{4}-\mathcal{W}_{|u| u}}{\left|\mathcal{W}_{|u| u}\right|}$ & $32.7737 \pm 3.0803$ & $0.4715 \pm 4.1298$ \\
\hline$\frac{\hat{\theta}_{5}-\mathcal{X}_{\mu}}{\left|\mathcal{X}_{\mu}\right|}$ & $-0.6667 \pm 0.0234$ & $0.0021 \pm 0.0640$ \\
\hline$\frac{\hat{\theta}_{6}-\mathcal{X}_{\kappa}}{\left|\mathcal{X}_{\kappa}\right|}$ & $0.8242 \pm 0.0764$ & $-0.0059 \pm 0.2312$ \\
\hline$\frac{\hat{\theta}_{7}-\mathcal{Y}_{v}}{\left|\mathcal{Y}_{v}\right|}$ & $0.0538 \pm 0.0071$ & $-0.0069 \pm 0.0734$ \\
\hline$\frac{\hat{\theta}_{8}-\mathcal{Y}_{u r}}{\left|\mathcal{Y}_{u r}\right|}$ & $0.5935 \pm 0.0380$ & $-0.0123 \pm 0.1121$ \\
\hline$\frac{\hat{\theta}_{9}-\mathcal{Y}_{|v| v}}{\left|\mathcal{Y}_{|v| v \mid}\right|}$ & $0.3263 \pm 0.0171$ & $-0.0159 \pm 0.1334$ \\
\hline$\frac{\hat{\theta}_{10}-\mathcal{W}_{|v| v}}{\left|\mathcal{W}_{|v| v}\right|}$ & $-0.7192 \pm 0.3408$ & $-0.0326 \pm 0.5997$ \\
\hline$\frac{\hat{\theta}_{11}-\mathcal{Y}_{|v| r}}{\left|\mathcal{Y}_{|v| r \mid}\right|}$ & $8.3754 \pm 1.7210$ & $0.9334 \pm 3.1922$ \\
\hline$\frac{\hat{\theta}_{12}-\mathcal{Y}_{\mu}}{\left|\mathcal{Y}_{\mu}\right|}$ & $-0.3325 \pm 0.0163$ & $0.0137 \pm 0.0871$ \\
\hline$\frac{\hat{\theta}_{13}-\mathcal{N}_{r}}{\left|\mathcal{N}_{r}\right|}$ & $-0.5806 \pm 0.0449$ & $-0.0016 \pm 0.0111$ \\
\hline$\frac{\hat{\theta}_{11}-\mathcal{N}_{u v}}{\left|\mathcal{N}_{u v}\right|}$ & $-4.1452 \pm 0.6798$ & $-0.1087 \pm 1.5434$ \\
\hline$\frac{\hat{\theta}_{15}-\mathcal{N}_{|v| v}}{\left|\mathcal{N}_{|v| v \mid}\right|}$ & $-7.9390 \pm 1.0842$ & $-0.0187 \pm 0.2091$ \\
\hline$\frac{\hat{\theta}_{16}-\mathcal{N}_{|v| r}}{\mid \mathcal{N}_{|v| r \mid}}$ & $-4.0171 \pm 0.8344$ & $-0.0024 \pm 0.1717$ \\
\hline$\frac{\hat{\theta}_{17}-\mathcal{W}_{u v}}{\left|\mathcal{W}_{u v}\right|}$ & $33.4305 \pm 34.5225$ & $5.1621 \pm 77.2216$ \\
\hline$\frac{\hat{\theta}_{18}-\mathcal{N}_{\mu}}{\left|\mathcal{N}_{\mu}\right|}$ & $4.5405 \pm 0.1145$ & $0.0097 \pm 0.0483$ \\
\hline
\end{tabular}


Table 6.5: The mean plus/minus one standard deviation of normalized estimation errors for two LS estimators not utilizing wind measurements. Data from the set of Monte Carlo runs with high wind speed was used for estimation.

\begin{tabular}{||c|c|c||}
\hline & $L S_{1}$ & $L S_{2}$ \\
\hline$\frac{\hat{\theta}_{1}-\mathcal{X}_{u}}{\left|\mathcal{X}_{u}\right|}$ & $0.0404 \pm 0.0009$ & $0.0565 \pm 0.0036$ \\
\hline$\frac{\hat{\theta}_{2}-\mathcal{X}_{r v}}{\left|\mathcal{X}_{r v}\right|}$ & $-0.8982 \pm 0.0032$ & $-0.7351 \pm 0.0148$ \\
\hline$\frac{\hat{\theta}_{3}-\left(\mathcal{X}_{|u| u}+\mathcal{W}_{|u| u}\right)}{\left|\mathcal{X}_{|u| u}+\mathcal{W}_{|u| u}\right|}$ & $1.0272 \pm 0.0094$ & $0.3862 \pm 0.0354$ \\
\hline$\frac{\hat{\theta}_{4}-\mathcal{X}_{\mu}}{\left|\mathcal{X}_{\mu}\right|}$ & $-0.9234 \pm 0.0042$ & $-0.7975 \pm 0.0211$ \\
\hline$\frac{\hat{\theta}_{5}-\mathcal{X}_{\kappa}}{\left|\mathcal{X}_{k}\right|}$ & $0.8834 \pm 0.0175$ & $1.2534 \pm 0.0694$ \\
\hline$\frac{\hat{\theta}_{6}-\mathcal{Y}_{v}}{\left|\mathcal{Y}_{v}\right|}$ & $0.2241 \pm 0.0013$ & $0.1949 \pm 0.0029$ \\
\hline$\frac{\hat{\theta}_{7}-\mathcal{Y}_{u r}}{\left|\mathcal{Y}_{u r}\right|}$ & $0.5046 \pm 0.0137$ & $0.9679 \pm 0.0233$ \\
\hline$\frac{\hat{\theta}_{8}-\left(\mathcal{Y}_{|v| v}-\mathcal{W}_{|v| v}\right)}{\left|\mathcal{Y}_{|v| v}-\mathcal{Y}_{|v| v}\right|}$ & $1.0588 \pm 0.0029$ & $0.6328 \pm 0.0124$ \\
\hline$\frac{\hat{\theta}_{9}-\mathcal{Y}_{|v| r}}{\left|\mathcal{Y}_{|v| r}\right|}$ & $5.2393 \pm 0.1217$ & $-0.9368 \pm 0.2149$ \\
\hline$\frac{\hat{\theta}_{10}-\mathcal{Y}_{\mu}}{\left|\mathcal{Y}_{\mu}\right|}$ & $-0.9864 \pm 0.0008$ & $-0.7088 \pm 0.0082$ \\
\hline$\frac{\hat{\theta}_{11}-\mathcal{N}_{r}}{\left|\mathcal{N}_{r}\right|}$ & $-0.3320 \pm 0.0141$ & $-0.5500 \pm 0.0308$ \\
\hline$\frac{\hat{\theta}_{12}-\left(\mathcal{N}_{u v}+\mathcal{W}_{u v}\right)}{\left|\mathcal{N}_{u v}+\mathcal{W}_{u v}\right|}$ & $-3.0330 \pm 0.1623$ & $1.2716 \pm 0.3710$ \\
\hline$\frac{\hat{\theta}_{13}-\mathcal{N}_{|v| v}}{\left|\mathcal{N}_{|v| v}\right|}$ & $1.2763 \pm 0.3242$ & $-12.0168 \pm 1.0420$ \\
\hline$\frac{\hat{\theta}_{14}-\mathcal{N}_{|v| r}}{\left|\mathcal{N}_{|v| r}\right|}$ & $-0.4307 \pm 0.2661$ & $-3.0029 \pm 0.6034$ \\
\hline$\frac{\hat{\theta}_{15}-\mathcal{N}_{\mu}}{\left|\mathcal{N}_{\mu}\right|}$ & $2.0552 \pm 0.0698$ & $3.9702 \pm 0.1036$ \\
\hline
\end{tabular}


Table 6.6: The mean plus/minus one standard deviation of normalized estimation errors for two IV estimators not utilizing wind measurements. Data from the set of Monte Carlo runs with high wind speed was used for estimation.

\begin{tabular}{||c|c|c||}
\hline & $I V_{1}$ & $I V_{2}$ \\
\hline$\frac{\hat{\theta}_{1}-\mathcal{X}_{u}}{\left|\mathcal{X}_{u}\right|}$ & $-6.2544 \pm 1.0798$ & $-0.1564 \pm 0.0299$ \\
\hline$\frac{\hat{\theta}_{2}-\mathcal{X}_{r v}}{\left|\mathcal{X}_{v v}\right|}$ & $1.1187 \pm 0.3285$ & $0.0117 \pm 0.0449$ \\
\hline$\frac{\hat{\theta}_{3}-\left(\mathcal{X}_{|u| u}+\mathcal{W}_{|u| u}\right)}{\left|\mathcal{X}_{|u| u}+\mathcal{W}_{|u| u}\right|}$ & $43.8002 \pm 7.5375$ & $1.0357 \pm 0.2102$ \\
\hline$\frac{\hat{\theta}_{4}-\mathcal{X}_{\mu}}{\left|\mathcal{X}_{\mu}\right|}$ & $11.3908 \pm 2.1581$ & $0.1748 \pm 0.0773$ \\
\hline$\frac{\hat{\theta}_{5}-\mathcal{X}_{k}}{\left|\mathcal{X}_{k}\right|}$ & $-45.7600 \pm 8.4159$ & $-0.7723 \pm 0.2732$ \\
\hline$\frac{\hat{\theta}_{6}-\mathcal{Y}_{v}}{\left|\mathcal{Y}_{v}\right|}$ & $0.6852 \pm 0.1407$ & $-0.1451 \pm 0.0475$ \\
\hline$\frac{\hat{\theta}_{7}-\mathcal{Y}_{u r}}{\left|\mathcal{Y}_{u r}\right|}$ & $-7.9334 \pm 1.4339$ & $0.4831 \pm 0.0763$ \\
\hline$\frac{\hat{\theta}_{8}-\left(\mathcal{Y}_{|v| v}-\mathcal{W}_{|v| v}\right)}{\left|\mathcal{Y}_{|v| v}-\mathcal{W}_{|v| v}\right|}$ & $2.4907 \pm 0.3081$ & $0.2072 \pm 0.0994$ \\
\hline$\frac{\hat{\theta}_{9}-\mathcal{Y}_{|v| r}}{\left|\mathcal{Y}_{|v| r}\right|}$ & $101.1160 \pm 15.9178$ & $-3.3620 \pm 0.6771$ \\
\hline$\frac{\hat{\theta}_{10}-\mathcal{Y}_{\mu}}{\left|\mathcal{Y}_{\mu}\right|}$ & $-1.9724 \pm 0.1508$ & $-0.0513 \pm 0.0682$ \\
\hline$\frac{\hat{\theta}_{11}-\mathcal{N}_{r}}{\left|\mathcal{N}_{r}\right|}$ & $-0.0265 \pm 0.0160$ & $-0.0009 \pm 0.0100$ \\
\hline$\frac{\hat{\theta}_{12}-\left(\mathcal{N}_{u v}+\mathcal{W}_{u v}\right)}{\left|\mathcal{N}_{u v}+\mathcal{W}_{u v}\right|}$ & $0.2630 \pm 0.1011$ & $-0.0010 \pm 0.1041$ \\
\hline$\frac{\hat{\theta}_{13}-\mathcal{N}_{|v| v}}{\left|\mathcal{N}_{|v| v}\right|}$ & $-0.1248 \pm 0.3742$ & $-0.0147 \pm 0.2372$ \\
\hline$\frac{\hat{\theta}_{14}-\mathcal{N}_{|v| r}}{\left|\mathcal{N}_{|v| r \mid}\right|}$ & $0.6394 \pm 0.2264$ & $0.0023 \pm 0.1697$ \\
\hline$\frac{\hat{\theta}_{15}-\mathcal{N}_{\mu}}{\left|\mathcal{N}_{\mu}\right|}$ & $-0.1070 \pm 0.0543$ & $0.0040 \pm 0.0410$ \\
\hline
\end{tabular}


Table 6.7: The mean plus/minus one standard deviation of normalized estimation errors for two estimators utilizing wind measurements. Data from the set of Monte Carlo runs with high wind speed was used for estimation.

\begin{tabular}{||c|c|c||}
\hline & $L S_{3}$ & $I V_{3}$ \\
\hline$\frac{\hat{\theta}_{1}-\mathcal{X}_{u}}{\left|\mathcal{X}_{u}\right|}$ & $0.0626 \pm 0.0044$ & $-0.0013 \pm 0.0291$ \\
\hline$\frac{\hat{\theta}_{2}-\mathcal{X}_{r v}}{\left|\mathcal{X}_{r v}\right|}$ & $-0.6230 \pm 0.0161$ & $0.0025 \pm 0.0438$ \\
\hline$\frac{\hat{\theta}_{3}-\mathcal{X}_{|u| u}}{\left|\mathcal{X}_{|u| u}\right|}$ & $0.1447 \pm 0.0473$ & $0.0057 \pm 0.2130$ \\
\hline$\frac{\hat{\theta}_{4}-\mathcal{W}_{|u| u}}{\left|\mathcal{W}_{|u| u}\right|}$ & $0.3638 \pm 0.0166$ & $-0.0012 \pm 0.0367$ \\
\hline$\frac{\hat{\theta}_{5}-\mathcal{X}_{\mu}}{\left|\mathcal{X}_{\mu}\right|}$ & $-0.6854 \pm 0.0246$ & $0.0039 \pm 0.0726$ \\
\hline$\frac{\hat{\theta}_{6}-\mathcal{X}_{\kappa}}{\left|\mathcal{X}_{k}\right|}$ & $1.2374 \pm 0.0867$ & $-0.0090 \pm 0.2633$ \\
\hline$\frac{\hat{\theta}_{7}-\mathcal{Y}_{v}}{\left|\mathcal{Y}_{v}\right|}$ & $0.0751 \pm 0.0059$ & $-0.0024 \pm 0.0534$ \\
\hline$\frac{\hat{\theta}_{8}-\mathcal{Y}_{u r}}{\mathcal{Y}_{u r} \mid}$ & $0.5884 \pm 0.0330$ & $-0.0099 \pm 0.1117$ \\
\hline$\frac{\hat{\theta}_{9}-\mathcal{Y}_{|v| v}}{\left|\mathcal{Y}_{|v| v \mid}\right|}$ & $0.2993 \pm 0.0174$ & $-0.0057 \pm 0.1239$ \\
\hline$\frac{\hat{\theta}_{10}-\mathcal{W}_{|v| v}}{\mid \mathcal{W}_{|v| v \mid}}$ & $-0.9430 \pm 0.2943$ & $0.0205 \pm 0.7147$ \\
\hline$\frac{\hat{\theta}_{11}-\mathcal{Y}_{|v| r}}{\left|\mathcal{Y}_{|v| r}\right|}$ & $0.3199 \pm 0.0158$ & $-0.0051 \pm 0.0758$ \\
\hline$\frac{\hat{\theta}_{12}-\mathcal{Y}_{\mu}}{\left|\mathcal{Y}_{\mu}\right|}$ & $-0.2973 \pm 0.0144$ & $0.0063 \pm 0.0802$ \\
\hline$\frac{\hat{\theta}_{13}-\mathcal{N}_{r}}{\left|\mathcal{N}_{r}\right|}$ & $-0.4985 \pm 0.0308$ & $-0.0010 \pm 0.0110$ \\
\hline$\frac{\hat{\theta}_{14}-\mathcal{N}_{u v}}{\left|\mathcal{N}_{u v}\right|}$ & $-1.3072 \pm 0.4833$ & $0.0027 \pm 0.1590$ \\
\hline$\frac{\hat{\theta}_{15}-\mathcal{N}_{|v| v}}{\left|\mathcal{N}_{|v| v \mid}\right|}$ & $-11.6011 \pm 1.0547$ & $-0.0180 \pm 0.2399$ \\
\hline$\frac{\hat{\theta}_{16}-\mathcal{N}_{|v| r}}{\left|\mathcal{N}_{|v| r \mid}\right|}$ & $-4.3462 \pm 0.6150$ & $0.0038 \pm 0.1849$ \\
\hline$\frac{\hat{\theta}_{17}-\mathcal{W}_{u v}}{\left|\mathcal{W}_{u v}\right|}$ & $-2.8341 \pm 0.3977$ & $-0.1005 \pm 6.0789$ \\
\hline$\frac{\hat{\theta}_{18}-\mathcal{N}_{\mu}}{\left|\mathcal{N}_{\mu}\right|}$ & $4.1584 \pm 0.1077$ & $0.0043 \pm 0.0418$ \\
\hline
\end{tabular}



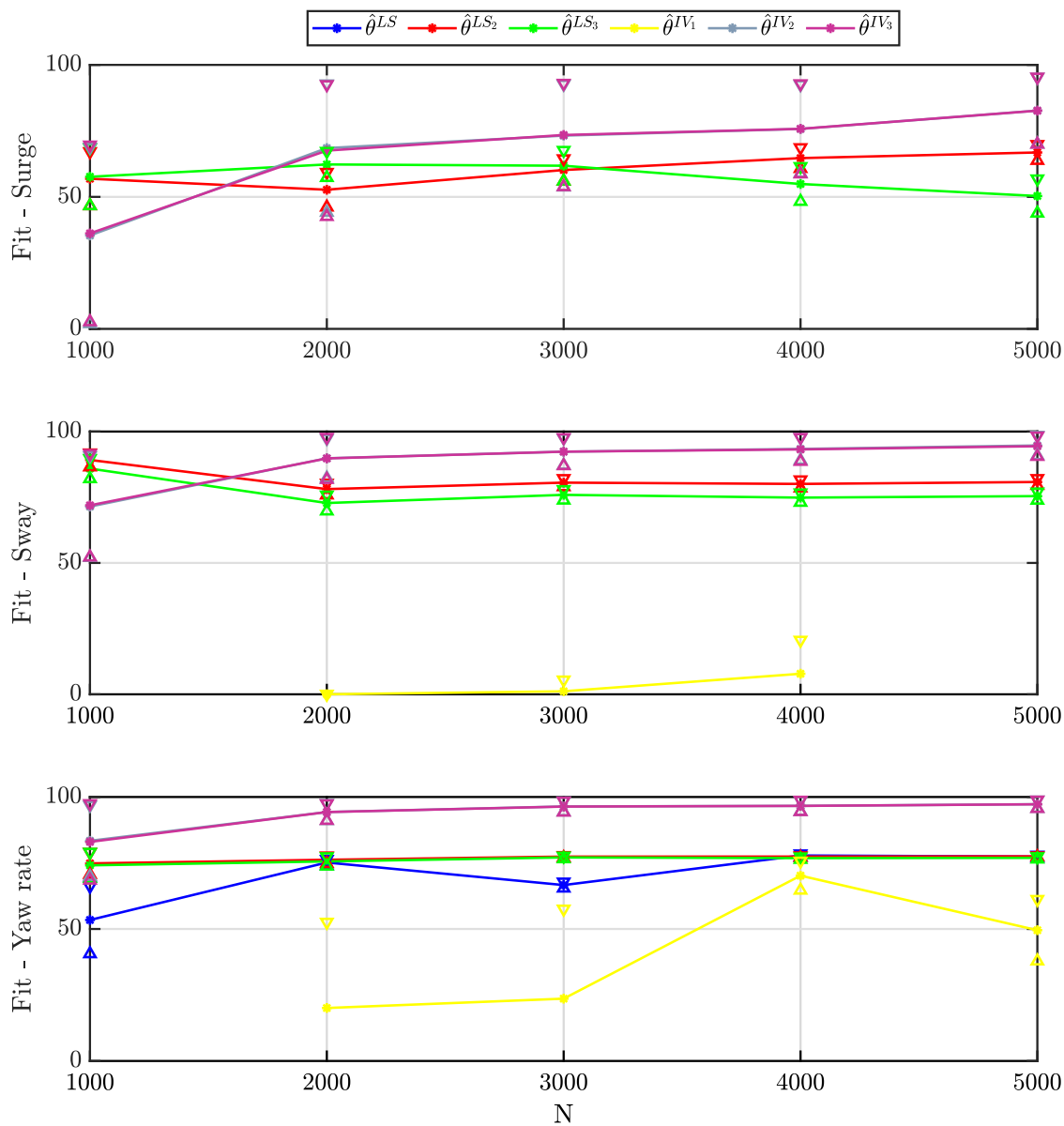

Figure 6.2: The average fit of models obtained from the six examined estimators for different values of $N$. Data with low wind speed was used for estimation. The figure only shows positive fit values, therefore the yellow and blue lines are sometimes missing. Also, the gray and magenta lines almost overlap which makes the gray line hard to see. The triangles indicate average model fit plus/minus one standard deviation. 

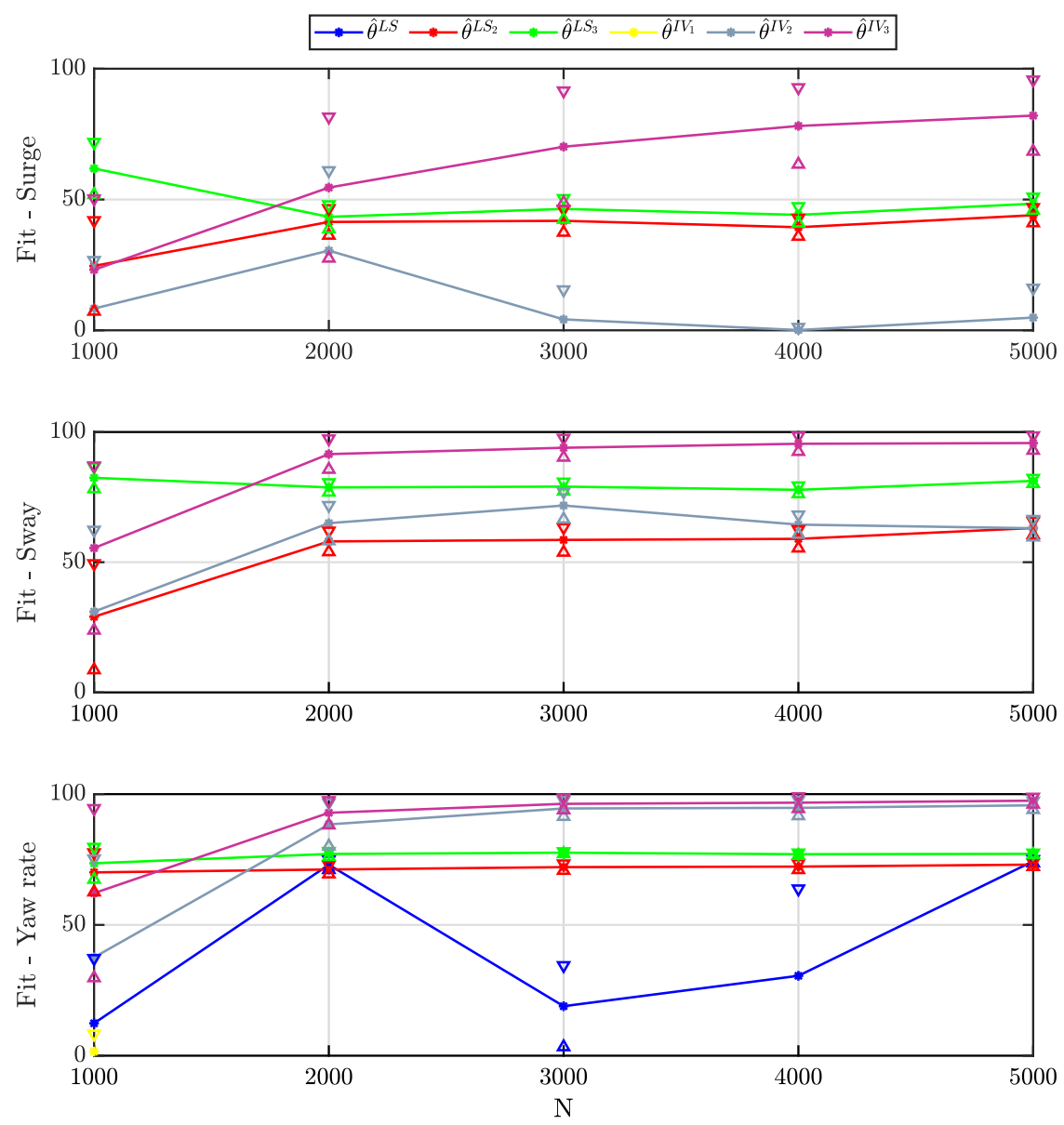

Figure 6.3: The average fit of models obtained from the six examined estimators for different values of $N$. Data with high wind speed was used for estimation. The figure only shows positive fit values, therefore the yellow and blue lines are sometimes missing. The triangles indicate average model fit plus/minus one standard deviation. 



\section{Experimental Study}

In this chapter, estimation results obtained using data from a full-scale marine vessel are presented. The data is provided by ABB, which is a Swiss-Swedish corporation that among other things works with automation technologies for marine vessels. First, the studied ship and the experiments are described briefly. Since no substantial ocean currents could be observed at the experiment location, the estimators do not need to account for disturbances of that type. Therefore, the estimators suggested in Chapter 5 are then simplified by removal of some redundant regressors. Finally, results from using the ship data for estimation of the surge-sway-yaw model (3.51) are presented.

\subsection{Experiment description}

The ship that was used to collect the data is roughly 30 meters long and has an actuator setup with two azimuth thrusters, $N_{a}=2$. The thrusters are mounted along the centerline, one at the front and one in the rear

$$
\begin{aligned}
\Delta_{x, 1} & =9, \\
\Delta_{x, 2} & =-9, \\
\Delta_{y, 1} & =0, \\
\Delta_{y, 2} & =0 .
\end{aligned}
$$

Notably, this thruster configurations makes it possible to excite the ship in sway without having a forward speed. 
For sensing, a GNSS receiver was used to provide measurements of the ship's position. The receiver collected new information once every second and had two antennas, which made it possible to also obtain estimates of the ship's yaw angle. Further, the ship was equipped with a propeller-based anemometer mounted on a weather vane for measuring wind speed and direction. The wind sensors collected data every five seconds and in order to obtain the same sampling frequency as the GNSS receiver, the wind measurements were padded under a zero-orderhold assumption. The actuator signals were sampled at a higher rate than the sensors but this was not utilized in the parameter estimation and the fact that the actuator signals sometimes changed in between the sampling points was neglected. To summarize, all the data was resampled at $T_{s}=1 \mathrm{~s}$.

The data was collected on two separate occasions with different weather conditions. One set of data was collected on a day with wind speeds of less than 3 $\mathrm{m} / \mathrm{s}$, i.e. light breeze by the definition in Fossen [2011]. On the other day of experiments, the wind speed was about $10 \mathrm{~m} / \mathrm{s}$, which corresponds to fresh breeze by the same set of definitions. On both these days, six shorter experiments were carried out with varying level of excitation in surge and sway. Each of the shorter experiments lasted for about 6-10 minutes. In total, the 12 batches of data consisted of 5840 samples which corresponds to roughly 97 minutes of experiment time.

In Figure 7.1, data from one of these short experiments is shown. For visualization purposes, the measurements from the GNSS receiver have been converted to a coordinate system with origin in the initial position of the ship. The anemometer measurements, $V_{q}$ and $\gamma_{q}$, constitute wind speed and wind angle relative to the speed and attitude of the ship, respectively, i.e. polar measurements in the $b$-frame as explained in Section 5.1.4. The fact that the measurements are relative can be seen by their tight connection to the ship's yaw angle. The two bottom plots show the actuator signals. The propellers both rotate with the same positive speed and the angle of the bow thruster is kept still while the angle of the stern thruster is varied. An exception is in the end where the bow thruster is used to decelerate the ship.

This actuation makes the ship go forward in a zig-zag manner which notably is not in agreement with the proposed idea of avoiding the origin. In other words, the experiments were not conducted in the way suggested in the earlier chapters of this work. Furthermore, all experiments were carried out at the same geographical location. In this region the water was essentially at standstill and no substantial ocean currents could be observed. These two facts of course significantly limits what hypotheses the experimental data can be expected to verify.

\subsection{Parameter estimation}

In Chapters 4 and 5, parameter estimation biases are discussed. To see the effects of such model errors on real data, it is necessary to have validation data which is collected independently of the estimation data. Since there is presumably a 

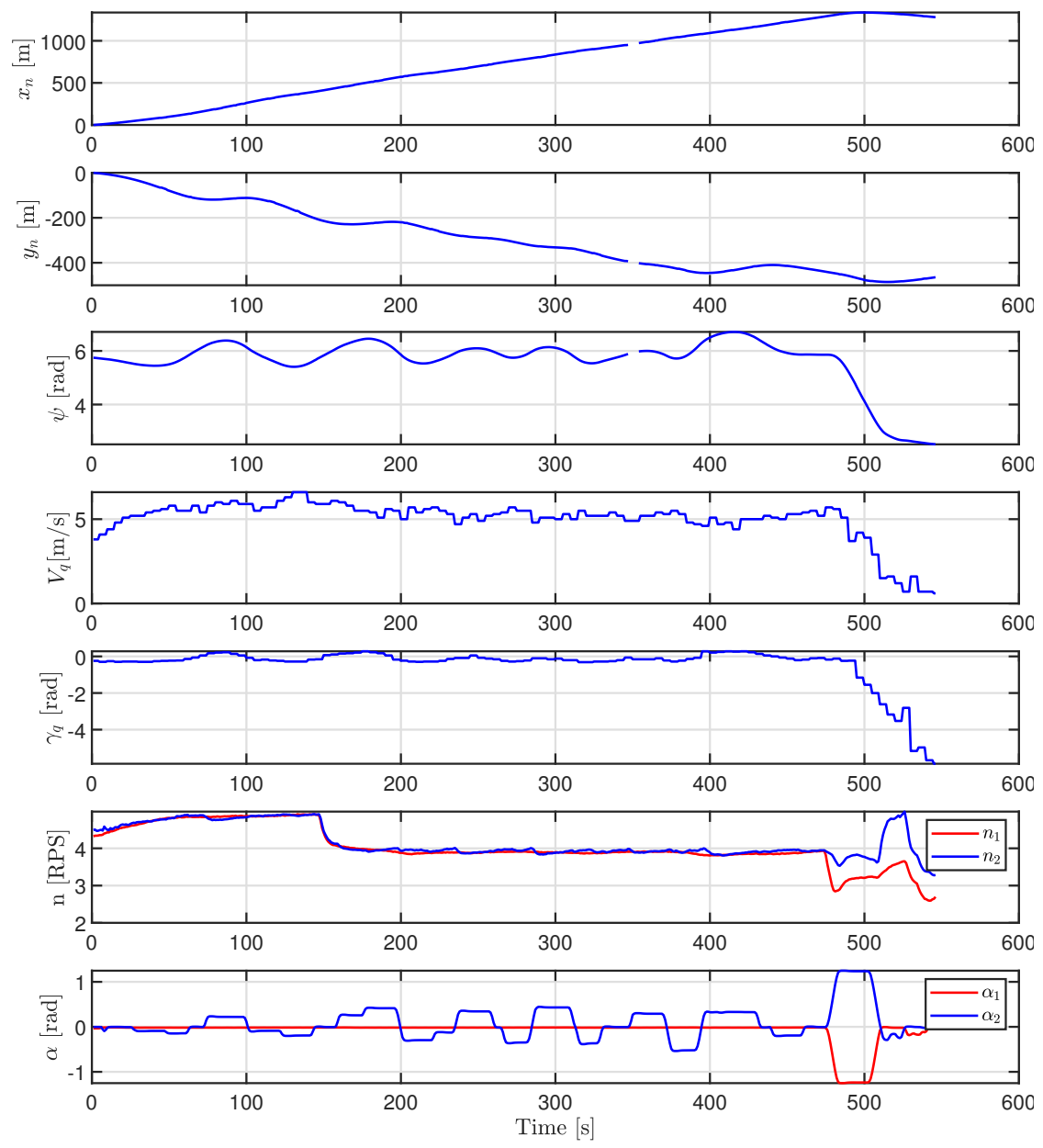

Figure 7.1: Example of a batch of measurement data from the less windy day. 
slowly changing deterministic trend in the wind disturbance, it is not sufficient to consider two in time adjacent experiments as independent. Therefore, it was decided to solely use the data from the most windy day for estimation and keep the data from the other day aside for validation purposes. All the data from the windy day was however not necessary for estimating the parameters of a model and smaller sets of estimation data were formed. Each set was made up by two experiments with surge as main excitaion and two experiments with sway as main excitation. This partitioning was chosen in order to have balanced sets of estimation data and avoid an uneven weighting of different DOF. There are nine ways of forming datasets in this way. Basing the analysis on multiple estimation datasets also gives the possibility of providing some statistical measures for the shown results. However, since the amount of experimental data is quite limited and the discussed effects are small with respect to other model errors, the shown results will be uncertain.

Six estimators were deemed relevant to compare in this experimental analysis, denoted $\hat{\theta}_{N}^{L S_{1}}, \hat{\theta}_{N}^{L S_{2}}, \hat{\theta}_{N}^{L S_{3}}, \hat{\theta}_{N}^{I V_{1}}, \hat{\theta}_{N}^{I V_{2}}, \hat{\theta}_{N}^{I V_{3}}$. These estimators roughly correspond to the ones analyzed in Chapter 6 . However, since there is no ocean-current disturbance present here, many of the bias-capturing regressors are redundant. This can be seen by inspecting the earlier shown asymptotic estimates (5.63), (5.66), (5.72), (5.81), (5.99), (5.104), (5.109) and noting that when $\bar{v}_{c, N S}=\bar{v}_{c, E W}=0$, many of the bias parameters are zero. In order to improve the variance properties of the estimators, the redundant regressors were removed. The estimators not utilizing wind measurements, $\hat{\theta}_{N}^{L S_{2}}$ and $\hat{\theta}_{N}^{I V_{2}}$, used predictors based on the regression vectors

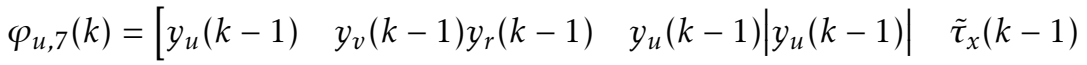

$$
\begin{aligned}
& y_{u}(k-1) \tilde{\tau}_{x}(k-1) \quad y_{u}(k-1) \cos \left(y_{\psi}(k-1)\right) \\
& \left.y_{u}(k-1) \sin \left(y_{\psi}(k-1)\right) \quad \cos ^{2}\left(y_{\psi}(k-1)\right) \sin \left(2 y_{\psi}(k-1)\right)\right]^{T} \text {, } \\
& \varphi_{v, 4}(k)=\left[\begin{array}{lll}
y_{v}(k-1) & y_{u}(k-1) y_{r}(k-1) \quad y_{v}(k-1)\left|y_{v}(k-1)\right|
\end{array}\right. \\
& y_{r}(k-1)\left|y_{v}(k-1)\right| \quad \tilde{\tau}_{y}(k-1) \quad \cos \left(y_{\psi}(k-1)\right) \quad \sin \left(y_{\psi}(k-1)\right) \\
& \left.\cos ^{2}\left(y_{\psi}(k-1)\right) \sin \left(2 y_{\psi}(k-1)\right)\right]^{T} \text {, }
\end{aligned}
$$

and

$$
\begin{aligned}
& \varphi_{r, 4}(k)=\left[y_{r}(k-1) \quad y_{u}(k-1) y_{v}(k-1) \quad y_{v}(k-1)\left|y_{v}(k-1)\right|\right. \\
& y_{r}(k-1)\left|y_{v}(k-1)\right| \quad \tilde{\tau}_{\psi}(k-1) \quad y_{v}(k-1) \cos \left(y_{\psi}(k-1)\right) \\
& y_{v}(k-1) \sin \left(y_{\psi}(k-1)\right) \quad y_{u}(k-1) \cos \left(y_{\psi}(k-1)\right) \\
& \left.y_{u}(k-1) \sin \left(y_{\psi}(k-1)\right) \quad \cos ^{2}\left(y_{\psi}(k-1)\right) \quad \sin \left(2 y_{\psi}(k-1)\right)\right]^{T} \text {. }
\end{aligned}
$$


Notably, in addition to removal of redundant regressors, these predictors were modified slightly. For example, to be in complete agreement with the analysis in Chapter 5, the regressors $|v(k-1)| \cos (\psi(k-1))$ and $|v(k-1)| \sin (\psi(k-1))$ should have been included in the sway predictor. However, including them did have a very negative impact on the resulting models and consequently they were replaced with the simpler regressors $\cos (\psi(k-1))$ and $\sin (\psi(k-1))$. Moreover, the absolute values and signum functions were removed from the bias-capturing regressors. Recall that in severe wind conditions, the experiment-design requirements, (5.7), (5.32), (5.68) and (5.69), are violated. The analysis of the estimators $\hat{\theta}_{N}^{L S_{2}}$ and $\hat{\theta}_{N}^{I V_{2}}$, in Chapter 5, does therefore not apply. The above shown predictors turned out to give the best results in the subsequent validations. The reason why the modifications improved the results is not yet understood

The estimators utilizing wind measurements, $\hat{\theta}_{N}^{L S_{3}}$ and $\hat{\theta}_{N}^{I V_{3}}$, do in this case not include any $\psi$-dependent regressors at all and the only difference between them and the estimators relying on basic predictors, $\hat{\theta}_{N}^{L S_{1}}$ and $\hat{\theta}_{N}^{I V_{1}}$, are one windmeasurement-dependent regressor in each DOF

$$
\begin{aligned}
& \varphi_{u, 8}(k)=\left[\begin{array}{lll}
y_{u}(k-1) & y_{v}(k-1) y_{r}(k-1) & y_{u}(k-1) \mid y_{u}(k-1)
\end{array} \mid\right. \\
& \left.y_{u_{q}}^{\prime}(k-1)\left|y_{u_{q}}^{\prime}(k-1)\right| \quad \tilde{\tau}_{x}(k-1) \quad y_{u}(k-1) \tilde{\tau}_{x}(k-1)\right]^{T} \text {, } \\
& \varphi_{v, 5}(k)=\left[y_{v}(k-1) \quad y_{u}(k-1) y_{r}(k-1) \quad y_{v}(k-1)\left|y_{v}(k-1)\right|\right. \\
& \left.y_{r}(k-1)\left|y_{v}(k-1)\right| \quad y_{v_{q}}^{\prime}(k-1)\left|y_{v_{q}}^{\prime}(k-1)\right| \quad \tilde{\tau}_{y}(k-1)\right]^{T} \text {, }
\end{aligned}
$$

and

$$
\begin{aligned}
& \varphi_{r, 5}(k)=\left[\begin{array}{lll}
y_{r}(k-1) & y_{u}(k-1) y_{v}(k-1) \quad y_{v}(k-1) \mid y_{v}(k-1)
\end{array} \mid\right. \\
& \left.y_{r}(k-1)\left|y_{v}(k-1)\right| \quad y_{u_{q}}^{\prime}(k-1) y_{v_{q}}^{\prime}(k-1) \quad \tilde{\tau}_{\psi}(k-1)\right]^{T} \text {. }
\end{aligned}
$$

Here, $y_{u_{q}}^{\prime}(k-1)$ and $y_{v_{q}}^{\prime}(k-1)$ are the artificial wind measurements in (5.43).

As earlier, the instruments of the IV estimators were generated based on simulation of nominal models. These nominal models were obtained by picking the corresponding LS parameter estimates, i.e. $\hat{\theta}_{N}^{L S_{i}}$ was used to simulate instruments for $\hat{\theta}_{N}^{I V_{i}}$ for $i=1,2,3$. In the simulations, the disturbances were assumed to be zero. This means that the wind measurements were not used. Since each batch of estimation data included data from multiple experiments, one instrument matrix

$$
\mathbf{Z}_{E}^{i}(k)=\left[\zeta_{E, u}^{i}(k) \quad \zeta_{E, v}^{i}(k) \quad \zeta_{E, r}^{i}(k)\right], E=1, \ldots N_{E},
$$

was formed for each experiment. This is in agreement with the discussion in Chapter 4. Further, as mentioned earlier the experiments were not conducted 
with excitation offset and for that reason there was no merit in removing the mean from the instruments. In practice, it turned out to be difficult to simulate the attitude of the ship because the simulated yaw angle eventually diverged from the measurements. Also, there is no easy way of simulating the wind velocity. Therefore, instead of simulated yaw angle and relative wind velocity, the corresponding measurements were used in the instrument vector. Notably, this violates the assumption made in Chapter 5 regarding the instrument vector being independent of the measurement noises. With these changes, no advantage of a refinement procedure could be observed and therefore only one IV-step was conducted.

In order to perform parameter estimation in the way discussed previously in the thesis, estimates of the ship's velocity states were needed. For this, the finite difference approximation (3.57) was used. Doing this for the batch of measured data shown in Figure 7.1, gives the velocity estimates in Figure 7.2. Finite-difference estimates were used both for parameter estimation and model validation. The validation was done by computing the normalized model fit, (6.12), between the simulated model output and the velocity estimates based on finite-difference approximations of the measurements. The output was simulated for one batch of validation data at a time, similar to the instrument generation. Unlike the simulations done for instrument generation, the outputs of the models obtained from the estimators $\hat{\theta}_{N}^{L S_{3}}$ and $\hat{\theta}_{N}^{I V_{3}}$, were generated using wind measurements. This means that it was not a pure simulation. For the models obtained from $\hat{\theta}_{N}^{L S_{2}}$ and $\hat{\theta}_{N}^{I V_{2}}$, it was however assumed that the disturbances were zero. This means that the bias-capturing regressors only were active in the estimation step.

The aforementioned datasets were used to estimate sets of model parameters and the average model fit plus/minus one standard deviation, obtained when the corresponding models are cross validated on data from the less windy day, are given in Table 7.1. The standard deviations are high and it is therefore hard to conclude anything with certainty. However, adding bias-capturing regressors in the predictor seems to improve the accuracy of the LS estimators. The same thing can not be said about the IV estimators, where the conventional IV estimator, $\hat{\theta}_{N}^{I V_{1}}$, also seems to perform well. Notably, there is no observable merit in using wind measurements in an IV estimator either. The only thing that can be seen is that the conventional LS estimator, $\hat{\theta}_{N}^{L S_{1}}$, seems to be performing worse than the other estimators in surge and about equally well in the other two DOF. Even this is however hard to conclude due to the high uncertainty.

In order to make it more clear that $\hat{\theta}_{N}^{L S_{1}}$ is not achieving as good results as the other estimators, a type of sign test was conducted. The results of this test are provided in Table 7.2, where it can be seen that the models obtained from $\hat{\theta}_{N}^{L S_{1}}$ have the lowest fit in surge for almost all the estimation datasets. Moreover, it can be observed that $\hat{\theta}_{N}^{L S_{1}}$ often gives worse performing models than the other two LS estimators in every DOF.

It is surprising that there is no notable merit in including wind measurements 

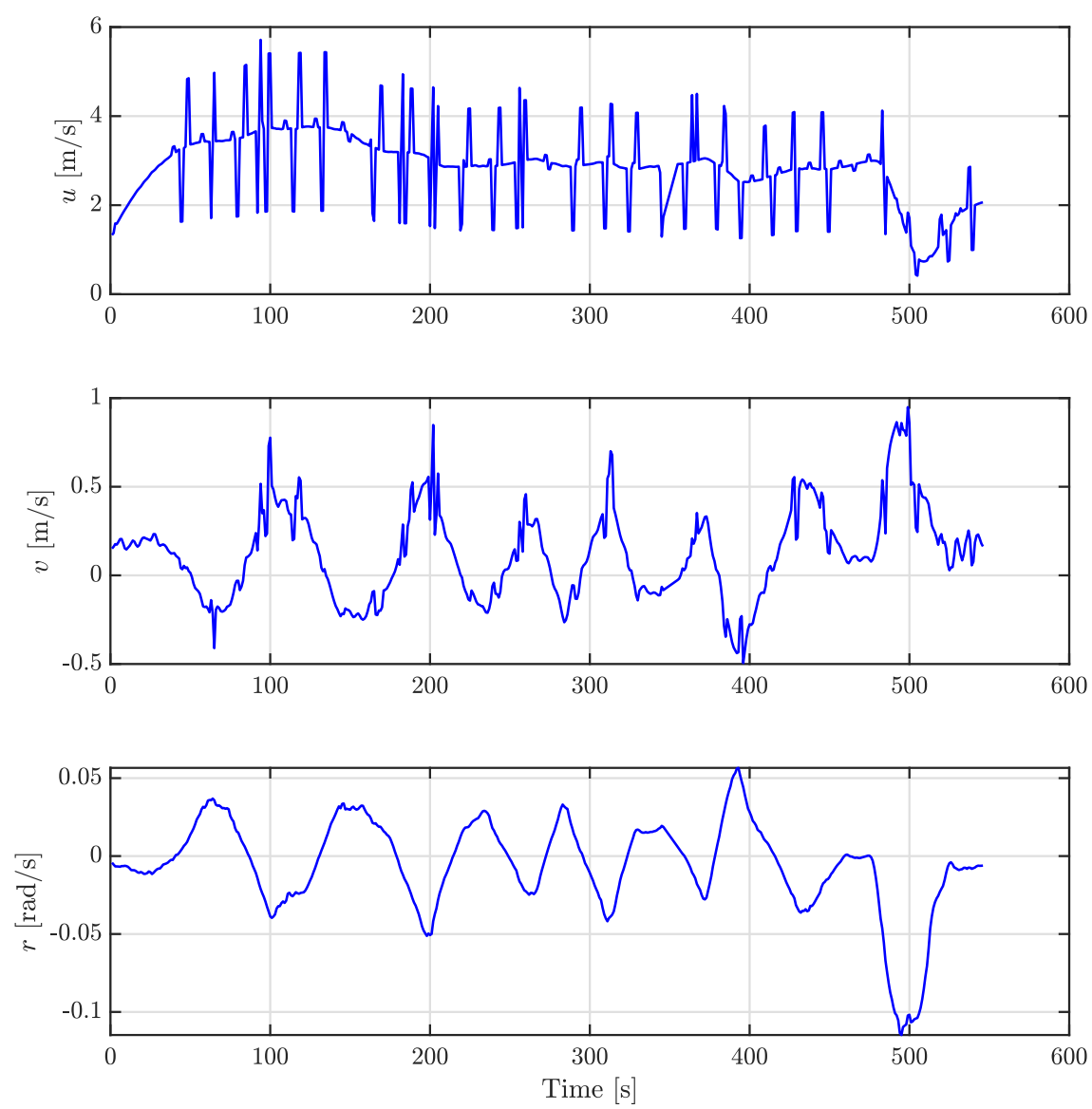

Figure 7.2: Finite-difference approximations of the velocity components following a batch of measurement data from the less windy day. Velocity estimates of this type were used both for estimation and validation. 
Table 7.1: The average fit of models obtained from the six examined estimators using 9 different estimation datasets from the most windy day, validated on all data from the less windy day. The average fit is given plus/minus one standard deviation in each DOF.

\begin{tabular}{||c|c|c|c||}
\hline Estimator & Fit - Surge & Fit - Sway & Fit - Yaw rate \\
\hline$\hat{\theta}_{N}^{L S_{1}}$ & $48.9206 \pm 6.0295$ & $44.6541 \pm 4.6111$ & $38.0489 \pm 9.7221$ \\
\hline$\hat{\theta}_{N}^{L S_{2}}$ & $57.3144 \pm 2.7117$ & $49.9672 \pm 2.7275$ & $43.5381 \pm 6.0683$ \\
\hline$\hat{\theta}_{N}^{L S_{3}}$ & $53.5811 \pm 4.5087$ & $48.3428 \pm 3.4570$ & $39.8135 \pm 9.1647$ \\
\hline$\hat{\theta}_{N}^{I V_{1}}$ & $55.7913 \pm 3.7346$ & $44.9494 \pm 3.0644$ & $45.1150 \pm 1.4595$ \\
\hline$\hat{\theta}_{N}^{I V_{2}}$ & $56.1931 \pm 4.7991$ & $45.9648 \pm 2.7519$ & $43.3887 \pm 2.6456$ \\
\hline$\hat{\theta}_{N}^{I V_{3}}$ & $56.0920 \pm 8.4091$ & $43.1615 \pm 6.0109$ & $43.1556 \pm 5.7910$ \\
\hline
\end{tabular}

Table 7.2: Sign-test comparison of models obtained using 9 different estimation datasets from the most windy day, validated on all data from the less windy day. The number of times each estimator gives a model with higher fit than the estimator $\hat{\theta}_{N}^{L S_{1}}$ is given in each DOF.

\begin{tabular}{||c|c|c|c||}
\hline Estimator & \#Wins - Surge & \#Wins - Sway & \#Wins - Yaw rate \\
\hline$\hat{\theta}_{N}^{L S_{2}}$ & $9 / 9$ & $9 / 9$ & $7 / 9$ \\
\hline$\hat{\theta}_{N}^{L S_{3}}$ & $9 / 9$ & $9 / 9$ & $9 / 9$ \\
\hline$\hat{\theta}_{N}^{I V_{1}}$ & $9 / 9$ & $3 / 9$ & $7 / 9$ \\
\hline$\hat{\theta}_{N}^{I V_{2}}$ & $8 / 9$ & $4 / 9$ & $6 / 9$ \\
\hline$\hat{\theta}_{N}^{I V_{3}}$ & $8 / 9$ & $4 / 9$ & $6 / 9$ \\
\hline
\end{tabular}


Table 7.3: Estimated aerodynamic drag coefficients obtained using 9 different estimation datasets from the most windy day. The estimates are given plus/minus one standard deviation.

\begin{tabular}{||c|c|c|c||}
\hline Estimator & $\mathcal{W}_{|u| u}$ & $\mathcal{W}_{|v| v}$ & $\mathcal{W}_{u v}$ \\
\hline$\hat{\theta}_{N}^{L S_{3}}$ & $(-9.38 \pm 2.22) \cdot 10^{-5}$ & $(-2.51 \pm 0.25) \cdot 10^{-4}$ & $(-2.32 \pm 0.25) \cdot 10^{-7}$ \\
\hline$\hat{\theta}_{N}^{I V_{3}}$ & $(-8.16 \pm 2.92) \cdot 10^{-5}$ & $(-2.99 \pm 0.49) \cdot 10^{-4}$ & $(-0.53 \pm 0.46) \cdot 10^{-7}$ \\
\hline
\end{tabular}

Table 7.4: Estimated linear hydrodynamic damping coefficients obtained using 9 different estimation datasets from the most windy day. The estimates are given plus/minus one standard deviation.

\begin{tabular}{||c|c|c|c||}
\hline Estimator & $\mathcal{X}_{u}$ & $\mathcal{Y}_{v}$ & $\mathcal{N}_{r}$ \\
\hline$\hat{\theta}_{N}^{L S_{3}}$ & $-0.0522 \pm 0.0030$ & $-0.1369 \pm 0.0134$ & $-0.0879 \pm 0.0220$ \\
\hline$\hat{\theta}_{N}^{I V_{3}}$ & $-0.0578 \pm 0.0026$ & $-0.3005 \pm 0.0236$ & $-0.1498 \pm 0.0165$ \\
\hline
\end{tabular}

for an IV estimator. The estimated aerodynamic damping coefficients from $\hat{\theta}_{N}^{L S_{3}}$ and $\hat{\theta}_{N}^{I V_{3}}$, following the estimation datasets used above, are given in Table 7.3. These coefficients attain negative values which is reasonable from a physical point of view and the corresponding standard deviations are small. However, when compared with the linear hydrodynamic damping coefficients in Table 7.4, the aerodynamic damping effects can be seen to be quite insignificant.

In Table 7.3 it can also be seen that the aerodynamic damping is notably bigger in sway. This is also reasonable from a physical standpoint, because the area which is exposed to wind in that direction is likely larger than the frontal area of the ship. In order to see a notable effect of the discussed methods another type of test was conducted based on this observation. There are three different ways of selecting a set of two experiments from the most windy day with sway as main excitation. These were used as estimation data and the three experiments with sway as main excitation from the less windy day were used as validation data. The resulting values of model fit in sway are provided in Table 7.5. In this special case it can be seen that the conventional IV estimator, $\hat{\theta}_{N}^{I V_{1}}$, yields less accurate models than the IV estimators with augmented predictors, $\hat{\theta}_{N}^{I V_{2}}$ and $\hat{\theta}_{N}^{I V_{3}}$. The same thing can be said about the conventional LS estimator, $\hat{\theta}_{N}^{L S_{1}}$, which gives worse models than the corresponding estimators with augmented predictors $\hat{\theta}_{N}^{L S_{2}}$ and $\hat{\theta}_{N}^{L S_{3}}$. These observations were verified in sign tests, similar to above. The results of those tests are given in Tables 7.6 and 7.7. The special case with a sway-specific model is of limited relevance in practice. Still, the results shown in 
Table 7.5: The average fit in sway of models obtained using 3 different estimation datasets with sway as main excitation from the most windy day, validated on data from the less windy day. The average fit is given plus/minus one standard deviation.

\begin{tabular}{||c|c||}
\hline Estimator & Fit - Sway \\
\hline$\hat{\theta}_{N}^{L S_{1}}$ & $50.3056 \pm 4.4771$ \\
\hline$\hat{\theta}_{N}^{L S_{2}}$ & $64.5389 \pm 2.8203$ \\
\hline$\hat{\theta}_{N}^{L S_{3}}$ & $62.2644 \pm 4.1303$ \\
\hline$\hat{\theta}_{N}^{I V_{1}}$ & $63.3711 \pm 8.3876$ \\
\hline$\hat{\theta}_{N}^{I V_{2}}$ & $71.9800 \pm 2.8652$ \\
\hline$\hat{\theta}_{N}^{I V_{3}}$ & $70.3522 \pm 3.4760$ \\
\hline
\end{tabular}

Tables 7.5, 7.6 and 7.7 give an indication that the discussed bias effects exist and that augmenting the predictor with wind-dependent regressors in some cases can improve the accuracy of an IV estimator. 
Table 7.6: Sign-test comparison of models obtained using 3 different estimation datasets from the most windy day with sway as main excitation, validated on data from the less windy day. The number of times each estimator gives a model with higher fit than $\hat{\theta}_{N}^{L S_{1}}$ in sway is given.

\begin{tabular}{||c|c||}
\hline Estimator & \#Wins - Sway \\
\hline$\hat{\theta}_{N}^{L S_{2}}$ & $3 / 3$ \\
\hline$\hat{\theta}_{N}^{L S_{3}}$ & $3 / 3$ \\
\hline$\hat{\theta}_{N}^{I V_{1}}$ & $3 / 3$ \\
\hline$\hat{\theta}_{N}^{I V_{2}}$ & $3 / 3$ \\
\hline$\hat{\theta}_{N}^{I V_{3}}$ & $3 / 3$ \\
\hline
\end{tabular}

Table 7.7: Sign-test comparison of models obtained using 3 different estimation datasets from the most windy day with sway as main excitation, validated on data from the less windy day. The number of times each estimator gives a model with higher fit than $\hat{\theta}_{N}^{I V_{1}}$ in sway is given.

\begin{tabular}{||c|c||}
\hline Estimator & \#Wins - Sway \\
\hline$\hat{\theta}_{N}^{L S_{1}}$ & $0 / 3$ \\
\hline$\hat{\theta}_{N}^{L S_{2}}$ & $2 / 3$ \\
\hline$\hat{\theta}_{N}^{L S_{3}}$ & $2 / 3$ \\
\hline$\hat{\theta}_{N}^{I V_{2}}$ & $3 / 3$ \\
\hline$\hat{\theta}_{N}^{I V_{3}}$ & $3 / 3$ \\
\hline
\end{tabular}





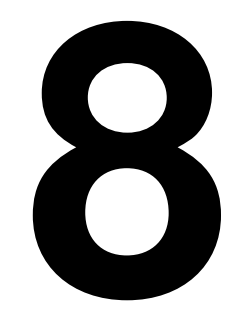

\section{Conclusions}

In this thesis, nonlinear system identification has been applied for marine applications. In particular, parameter estimators for a special type on nonlinear regression models called second-order modulus models have been developed. This is primarily of interest for maritime applications, where these type of models are common but the usefulness of the results can extend to other applications as well.

The trade-off between variance and bias is unavoidable in system identification and in this work the primary focus has been to asymptotically eliminate the latter. For a company developing controllers for ships, it is of importance to have ways of obtaining accurate simulations models. However, it is also of relevance that the data acquisition is not too time consuming because performing experiments is expensive. In a situation with limited amount of estimation data, an inconsistent estimator might very well give better performing models than a consistent one. The simulation examples in Section 6.3 illustrate this. The estimators using predictors with augmented regression vectors suggested in Section 5.2, have more than twice as many parameters as the estimators using basic predictors. This adds complexity to the model structure and a natural side effect of that is higher variance in the parameter estimates. One possible way of reducing the variance is to enforce that some of the bias terms are zero, already in the experiment design. Thereby, the corresponding regressors would not have to be included in the predictor. As seen in Section 5.1, this can be done in the case where the ship is moving along a straight-line path. Removing all the estimation bias for a 3-DOF model with cross terms in this way, is perhaps not feasible. Enforcing that some terms are zero could however improve the variance properties of the estimators. This is a possible area of future work.

Moreover, the results in this thesis are based on the assumption that the absolute velocity states are measured directly. In practice it is more common that only 
some components of the state vector are available immediately, whereas other components must be obtained by filtering techniques. This leads on to discussions like the one in Section 3.6. Similar problems arise when wind sensors are not mounted onboard the ship and also when weather vanes are used, regardless of where they are mounted. The root of the problem lies in the uncertainty of a mapping from one coordinate system to another that follows an uncertain angle measurement. In theory, the model could instead be represented with $n$-frame velocities or cast on polar form. These are not the conventional ways of expressing ship models but they constitute interesting alternatives.

The results using real data shown in Chapter 7 are not at all as clear as the results shown in Chapter 6, that were obtained using simulated data. In fact, no benefit of adding bias-capturing regressors can be observed for an IV estimator when real data is used for estimation, unless only sway motion is considered. There are multiple plausible explanations for this. The most likely reason is perhaps that there are no observable ocean currents present at the experiment location. The forces and moments caused by aerodynamic drag enter the system in ways that are similar to their hydrodynamic counterparts. However, the aerodynamic effects seem to be very small in comparison, even in quite severe wind conditions. Furthermore, the experiments carried out to collect data were not done in the way proposed in this thesis. Due to these two reasons, it is possible that the suggested approach proves useful in another situation.

A third plausible explanation is that there are structural model errors caused by the model not being able to describe the data, which are more impactful than the discussed estimation bias. Techniques based on nonlinear blackbox modelling are increasing in popularity and it would be interesting to see how well such a method performs in comparison to the greybox approach taken in this work. It is then possible to have a very flexible model structure but a potential drawback is the lack of means to deal with environmental disturbances. A two-step solution where a greybox model is first identified for the sake of capturing disturbance characteristics and the estimated disturbance properties in a second step are used as input to a blackbox-model estimator, might therefore be beneficial.

Finally, there is a close connection between the work in Chapter 5 and design of disturbance observers. It would be interesting to see how accurately the environmental disturbance properties can be estimated using attitude-dependent regressors in the case where a model of the undisturbed system is already known. 


\section{Bibliography}

Mohamed Abdalmoaty. Identification of stochastic nonlinear dynamical models using estimating functions. PhD thesis, KTH Royal Institute of Technology, Stockholm, Sweden, 2019.

Martin A. Abkowitz. Lectures on ship hydrodynamics - steering and manoeuvrability. Technical Report HY-5, Hydro- and Aerodynamics Laboratory, Lyngby, Denmark, 1964.

Mogens Blanke. Ship propulsion losses related to automatic steering and prime mover control. PhD thesis, Technical University of Denmark, Lyngby, Denmark, 1981.

Torsten Bohlin. Practical grey-box process identification: theory and applications. Springer Science \& Business Media, London, U.K., 2006.

Pablo M. Carrica, Farzad Ismail, Mark Hyman, Shanti Bhushan, and Frederick Stern. Turn and zigzag maneuvers of a surface combatant using a URANS approach with dynamic overset grids. Journal of Marine Science and Technology, 18(2):166-181, 2013.

Manuel Haro Casado, Ramón Ferreiro, and Francisco J. Velasco. Identification of nonlinear ship model parameters based on the turning circle test. Journal of Ship Research, 51(2):174-181, 2007.

K. K. Fedyaevsky and G. V. Sobolev. Control and stability in ship design. State Union Ship-building House, St. Petersburg, Russia, 1964.

Thor I. Fossen. Guidance and control of ocean vehicles. John Wiley \& Sons Inc, Chichester, U.K., 1994.

Thor I. Fossen. Handbook of marine craft hydrodynamics and motion control. John Wiley \& Sons, Chichester, U.K., 2011.

Thor I. Fossen, Svein I. Sagatun, and Asgeir J. Sørensen. Identification of dynamically positioned ships. Journal of Control Engineering Practice, 4(3):369-376, 1996. 
Damien Garcia. Robust smoothing of gridded data in one and higher dimensions with missing values. Computational Statistics \& Data Analysis, 54(4):1167$1178,2010$.

Michel Gevers, Alexandre Sanfelice Bazanella, Xavier Bombois, and Ljubisa Miskovic. Identification and the information matrix: how to get just sufficiently rich? IEEE Transactions on Automatic Control, 54(12):2828-2840, 2009.

Marion Gilson, Hugues Garnier, Peter C. Young, and Paul Van den Hof. Optimal instrumental variable method for closed-loop identification. IET Control Theory \& Applications, 5(10):1147-1154, 2011.

Mohinder S. Grewal and Keith Glover. Identifiability of linear and nonlinear dynamical systems. IEEE Transactions on Automatic Control, 21(6):833-837, 1976.

Øyvind Hegrenæs. Autonomous navigation for underwater vehicles. PhD thesis, Norwegian University of Science and Technology, Trondheim, Norway, 2010.

Elias Revestido Herrero and Francisco J. Velasco Gonzalez. Two-step identification of non-linear manoeuvring models of marine vessels. Ocean Engineering, 53:72-82, 2012.

Vladislav Kopman, Jeffrey Laut, Francesco Acquaviva, Alessandro Rizzo, and Maurizio Porfiri. Dynamic modeling of a robotic fish propelled by a compliant tail. IEEE Journal of Oceanic Engineering, 40(1):209-221, 2015.

Roger Larsson. Flight test system identification. PhD thesis, Linköping University, Linköping, Sweden, 2019.

Jonas Linder. Indirect system identification for unknown input problems: With applications to ships. PhD thesis, Linköping University, Linköping, Sweden, 2017.

Lennart Ljung. System identification: theory for the user (2nd edition). Prentice Hall, Upper Saddle River, N. J., 1999.

Lennart Ljung. Perspectives on system identification. Annual Reviews in Control, 34(1):1-12, 2010.

Fredrik Ljungberg and Martin Enqvist. Obtaining consistent parameter estimators for second-order modulus models. IEEE Control Systems Letters, 3(4): 781-786, 102019.

Fredrik Ljungberg and Martin Enqvist. Consistent parameter estimators for second-order modulus systems with non-additive disturbances. In Proceedings of the 21st IFAC World Congress, Berlin, Germany, 2020 (to appear). 
Weilin Luo, C. Guedes Soares, and Zaojian Zou. Parameter identification of ship maneuvering model based on support vector machines and particle swarm optimization. Journal of Offshore Mechanics and Arctic Engineering, 138(3), 2016. Art. no. 031101.

Nicolas Minorsky. Directional stability of automatically steered bodies. Journal of the American Society for Naval Engineers, 34(2):280-309, 1922.

Nils H. Norrbin. Theory and observation on the use of a mathematical model for ship maneuvering in deep and confined waters. In Proceedings of the 8th symposium on naval hydrodynamics, pages 807--904, Pasadena, California, 1970.

Tristan Perez. Ship motion control: course keeping and roll stabilisation using rudder and fins. Springer-Verlag, London, U.K., 2005.

Tristan Perez, Tim Mak, Tony Armstrong, Andrew Ross, and Thor I Fossen. Validation of a 4DOF manoeuvring model of a high-speed vehicle-passenger trimaran. In Proceedings of the 9th International Conference on Fast Sea Transportation FAST2007, pages 23-27, Shanghai, China, 2007.

Thomas B. Schön, Adrian Wills, and Brett Ninness. System identification of nonlinear state-space models. Automatica, 47(1):39-49, 2011.

Johan Schoukens and Lennart Ljung. Nonlinear system identification: A useroriented roadmap. IEEE Control Systems Magazine, 39(6):28-99, 2019.

Roger Skjetne, Øyvind N. Smogeli, and Thor I. Fossen. A nonlinear ship manoeuvering model: Identification and adaptive control with experiments for a model ship. Modeling, Identification and Control, 25(1):3-27, 2004.

SNAME. The society of naval architects and marine engineers. Nomenclature for treating the motion of a submerged body through a fluid. Technical and Research Bulletin No 1-5, 1950.

Torsten Söderström. Errors-in-variables methods in system identification. Automatica, 43(6):939-958, 2007.

Torsten Söderström and Petre Stoica. System identification. Prentice Hall, Englewood Cliffs, N. J., 1989.

Serge Sutulo and C. Guedes Soares. An algorithm for offline identification of ship manoeuvring mathematical models from free-running tests. Ocean Engineering, 79:10-25, 2014.

Stéphane Thil, Marion Gilson, and Hugues Garnier. On instrumental variablebased methods for errors-in-variables model identification. In Proceedings of the 17th IFAC World Congress, pages 426-431, Seoul, Korea, 2008.

Job Van Amerongen. Adaptive steering of ships-a model reference approach. Automatica, 20(1):3-14, 1984. 
Hyeon Kyu Yoon and Key Pyo Rhee. Identification of hydrodynamic coefficients in ship maneuvering equations of motion by estimation-before-modeling technique. Ocean Engineering, 30(18):2379-2404, 2003.

Peter C. Young. The refined instrumental variable method. Journal Européen des Systemes Automatisés, 42(2-3):149-179, 2008.

Wei-Wu Zhou and Mogens Blanke. Identification of a class of non-linear state space models using RPE techniques. IEEE Transactions on Automatic Control, 34(3):312-316, 1989. 


\section{Licentiate Theses \\ Division of Automatic Control \\ Linköping University}

P. Andersson: Adaptive Forgetting through Multiple Models and Adaptive Control of Car Dynamics. Thesis No. 15, 1983.

B. Wahlberg: On Model Simplification in System Identification. Thesis No. 47, 1985.

A. Isaksson: Identification of Time Varying Systems and Applications of System Identification to Signal Processing. Thesis No. 75, 1986.

G. Malmberg: A Study of Adaptive Control Missiles. Thesis No. 76, 1986.

S. Gunnarsson: On the Mean Square Error of Transfer Function Estimates with Applications to Control. Thesis No. 90, 1986.

M. Viberg: On the Adaptive Array Problem. Thesis No. 117, 1987.

K. Ståhl: On the Frequency Domain Analysis of Nonlinear Systems. Thesis No. 137, 1988.

A. Skeppstedt: Construction of Composite Models from Large Data-Sets. Thesis No. 149, 1988.

P. A. J. Nagy: MaMiS: A Programming Environment for Numeric/Symbolic Data Processing. Thesis No. 153, 1988.

K. Forsman: Applications of Constructive Algebra to Control Problems. Thesis No. 231, 1990.

I. Klein: Planning for a Class of Sequential Control Problems. Thesis No. 234, 1990.

F. Gustafsson: Optimal Segmentation of Linear Regression Parameters. Thesis No. 246, 1990.

H. Hjalmarsson: On Estimation of Model Quality in System Identification. Thesis No. 251, 1990.

S. Andersson: Sensor Array Processing; Application to Mobile Communication Systems and Dimension Reduction. Thesis No. 255, 1990.

K. Wang Chen: Observability and Invertibility of Nonlinear Systems: A Differential Algebraic Approach. Thesis No. 282, 1991.

J. Sjöberg: Regularization Issues in Neural Network Models of Dynamical Systems. Thesis No. 366, 1993.

P. Pucar: Segmentation of Laser Range Radar Images Using Hidden Markov Field Models. Thesis No. 403, 1993.

H. Fortell: Volterra and Algebraic Approaches to the Zero Dynamics. Thesis No. 438, 1994.

T. McKelvey: On State-Space Models in System Identification. Thesis No. 447, 1994.

T. Andersson: Concepts and Algorithms for Non-Linear System Identifiability. Thesis No. 448, 1994.

P. Lindskog: Algorithms and Tools for System Identification Using Prior Knowledge. Thesis No. 456, 1994.

J. Plantin: Algebraic Methods for Verification and Control of Discrete Event Dynamic Systems. Thesis No. 501, 1995.

J. Gunnarsson: On Modeling of Discrete Event Dynamic Systems, Using Symbolic Algebraic Methods. Thesis No. 502, 1995.

A. Ericsson: Fast Power Control to Counteract Rayleigh Fading in Cellular Radio Systems. Thesis No. 527, 1995.

M. Jirstrand: Algebraic Methods for Modeling and Design in Control. Thesis No. 540, 1996.

K. Edström: Simulation of Mode Switching Systems Using Switched Bond Graphs. Thesis No. 586, 1996. 
J. Palmqvist: On Integrity Monitoring of Integrated Navigation Systems. Thesis No. 600, 1997.

A. Stenman: Just-in-Time Models with Applications to Dynamical Systems. Thesis No. 601, 1997.

M. Andersson: Experimental Design and Updating of Finite Element Models. Thesis No. 611, 1997.

U. Forssell: Properties and Usage of Closed-Loop Identification Methods. Thesis No. 641, 1997.

M. Larsson: On Modeling and Diagnosis of Discrete Event Dynamic systems. Thesis No. 648, 1997.

N. Bergman: Bayesian Inference in Terrain Navigation. Thesis No. 649, 1997.

V. Einarsson: On Verification of Switched Systems Using Abstractions. Thesis No. 705, 1998.

J. Blom, F. Gunnarsson: Power Control in Cellular Radio Systems. Thesis No. 706, 1998.

P. Spångéus: Hybrid Control using LP and LMI methods - Some Applications. Thesis No. 724, 1998.

M. Norrlöf: On Analysis and Implementation of Iterative Learning Control. Thesis No. 727, 1998.

A. Hagenblad: Aspects of the Identification of Wiener Models. Thesis No. 793, 1999.

F. Tjärnström: Quality Estimation of Approximate Models. Thesis No. 810, 2000.

C. Carlsson: Vehicle Size and Orientation Estimation Using Geometric Fitting. Thesis No. 840, 2000.

J. Löfberg: Linear Model Predictive Control: Stability and Robustness. Thesis No. 866, 2001.

O. Härkegård: Flight Control Design Using Backstepping. Thesis No. 875, 2001.

J. Elbornsson: Equalization of Distortion in A/D Converters. Thesis No. 883, 2001.

J. Roll: Robust Verification and Identification of Piecewise Affine Systems. Thesis No. 899, 2001.

I. Lind: Regressor Selection in System Identification using ANOVA. Thesis No. 921, 2001.

R. Karlsson: Simulation Based Methods for Target Tracking. Thesis No. 930, 2002.

P.-J. Nordlund: Sequential Monte Carlo Filters and Integrated Navigation. Thesis No. 945, 2002.

M. Östring: Identification, Diagnosis, and Control of a Flexible Robot Arm. Thesis No. 948, 2002.

C. Olsson: Active Engine Vibration Isolation using Feedback Control. Thesis No. 968, 2002.

J. Jansson: Tracking and Decision Making for Automotive Collision Avoidance. Thesis No. 965, 2002.

N. Persson: Event Based Sampling with Application to Spectral Estimation. Thesis No. 981, 2002.

D. Lindgren: Subspace Selection Techniques for Classification Problems. Thesis No. 995, 2002.

E. Geijer Lundin: Uplink Load in CDMA Cellular Systems. Thesis No. 1045, 2003.

M. Enqvist: Some Results on Linear Models of Nonlinear Systems. Thesis No. 1046, 2003.

T. Schön: On Computational Methods for Nonlinear Estimation. Thesis No. 1047, 2003.

F. Gunnarsson: On Modeling and Control of Network Queue Dynamics. Thesis No. 1048, 2003.

S. Björklund: A Survey and Comparison of Time-Delay Estimation Methods in Linear Systems. Thesis No. 1061, 2003. 
M. Gerdin: Parameter Estimation in Linear Descriptor Systems. Thesis No. 1085, 2004.

A. Eidehall: An Automotive Lane Guidance System. Thesis No. 1122, 2004.

E. Wernholt: On Multivariable and Nonlinear Identification of Industrial Robots. Thesis No. 1131, 2004.

J. Gillberg: Methods for Frequency Domain Estimation of Continuous-Time Models. Thesis No. 1133, 2004.

G. Hendeby: Fundamental Estimation and Detection Limits in Linear Non-Gaussian Systems. Thesis No. 1199, 2005.

D. Axehill: Applications of Integer Quadratic Programming in Control and Communication. Thesis No. 1218, 2005.

J. Sjöberg: Some Results On Optimal Control for Nonlinear Descriptor Systems. Thesis No. 1227, 2006.

D. Törnqvist: Statistical Fault Detection with Applications to IMU Disturbances. Thesis No. 1258, 2006.

H. Tidefelt: Structural algorithms and perturbations in differential-algebraic equations. Thesis No. 1318, 2007.

S. Moberg: On Modeling and Control of Flexible Manipulators. Thesis No. 1336, 2007.

J. Wallén: On Kinematic Modelling and Iterative Learning Control of Industrial Robots. Thesis No. 1343, 2008.

J. Harju Johansson: A Structure Utilizing Inexact Primal-Dual Interior-Point Method for Analysis of Linear Differential Inclusions. Thesis No. 1367, 2008.

J. D. Hol: Pose Estimation and Calibration Algorithms for Vision and Inertial Sensors. Thesis No. 1370, 2008.

H. Ohlsson: Regression on Manifolds with Implications for System Identification. Thesis No. 1382, 2008.

D. Ankelhed: On low order controller synthesis using rational constraints. Thesis No. 1398, 2009.

P. Skoglar: Planning Methods for Aerial Exploration and Ground Target Tracking. Thesis No. 1420, 2009.

C. Lundquist: Automotive Sensor Fusion for Situation Awareness. Thesis No. 1422, 2009.

C. Lyzell: Initialization Methods for System Identification. Thesis No. 1426, 2009.

R. Falkeborn: Structure exploitation in semidefinite programming for control. Thesis No. 1430, 2010.

D. Petersson: Nonlinear Optimization Approaches to $\mathcal{H}_{2}$-Norm Based LPV Modelling and Control. Thesis No. 1453, 2010.

Z. Sjanic: Navigation and SAR Auto-focusing in a Sensor Fusion Framework. Thesis No. 1464, 2011.

K. Granström: Loop detection and extended target tracking using laser data. Thesis No. 1465, 2011.

J. Callmer: Topics in Localization and Mapping. Thesis No. 1489, 2011.

F. Lindsten: Rao-Blackwellised particle methods for inference and identification. Thesis No. 1480, 2011.

M. Skoglund: Visual Inertial Navigation and Calibration. Thesis No. 1500, 2011.

S. Khoshfetrat Pakazad: Topics in Robustness Analysis. Thesis No. 1512, 2011.

P. Axelsson: On Sensor Fusion Applied to Industrial Manipulators. Thesis No. 1511, 2011.

A. Carvalho Bittencourt: On Modeling and Diagnosis of Friction and Wear in Industrial Robots. Thesis No. 1516, 2012.

P. Rosander: Averaging level control in the presence of frequent inlet flow upsets. Thesis No. 1527, 2012. 
N. Wahlström: Localization using Magnetometers and Light Sensors. Thesis No. 1581, 2013.

R. Larsson: System Identification of Flight Mechanical Characteristics. Thesis No. 1599, 2013.

Y. Jung: Estimation of Inverse Models Applied to Power Amplifier Predistortion. Thesis No. 1605, 2013.

M. Syldatk: On Calibration of Ground Sensor Networks. Thesis No. 1611, 2013.

M. Roth: Kalman Filters for Nonlinear Systems and Heavy-Tailed Noise. Thesis No. 1613, 2013.

D. Simon: Model Predictive Control in Flight Control Design - Stability and Reference Tracking. Thesis No. 1642, 2014.

J. Dahlin: Sequential Monte Carlo for inference in nonlinear state space models. Thesis No. 1652, 2014.

M. Kok: Probabilistic modeling for positioning applications using inertial sensors. Thesis No. 1656, 2014.

J. Linder: Graybox Modelling of Ships Using Indirect Input Measurements. Thesis No. 1681, 2014.

G. Mathai: Direction of Arrival Estimation of Wideband Acoustic Wavefields in a Passive Sensing Environment. Thesis No. 1721, 2015.

I. Nielsen: On Structure Exploiting Numerical Algorithms for Model Predictive Control. Thesis No. 1727, 2015.

C. Veibäck: Tracking of Animals Using Airborne Cameras. Thesis No. 1761, 2016.

N. Evestedt: Sampling Based Motion Planning for Heavy Duty Autonomous Vehicles. Thesis No. 1762, 2016.

H. Nyqvist: On Pose Estimation in Room-Scaled Environments. Thesis No. 1765, 2016.

Y. Zhao: Position Estimation in Uncertain Radio Environments and Trajectory Learning. Thesis No. 1772, 2017.

P. Kasebzadeh: Parameter Estimation for Mobile Positioning Applications. Thesis No. 1786, 2017.

K. Radnosrati: On Timing-Based Localization in Cellular Radio Networks. Thesis No. 1808, 2018.

G. Lindmark: Methods and Algorithms for Control Input Placement in Complex Networks. Thesis No. 1814, 2018.

M. Lindfors: Frequency Tracking for Speed Estimation. Thesis No. 1815, 2018.

D. Ho: Some results on closed-loop identification of quadcopters. Thesis No. 1826, 2018.

O. Ljungqvist: On motion planning and control for truck and trailer systems. Thesis No. 1832, 2019.

P. Boström-Rost: On Informative Path Planning for Tracking and Surveillance. Thesis No. 1838, 2019.

K. Bergman: On Motion Planning Using Numerical Optimal Control. Thesis No. 1843, 2019.

M. Klingspor: Low-rank optimization in system identification. Thesis No. 1855, 2019.

A. Bergström: Timing-Based Localization using Multipath Information. Thesis No. 1867, 2019. 


\section{FACULTY OF SCIENCE AND ENGINEERING}

Linköping studies in science and technology. Licentiate Thesis No. 1880

Department of Electrical Engineering

Linköping University

SE-581 83 Linköping, Sweden

www.liu.se 